

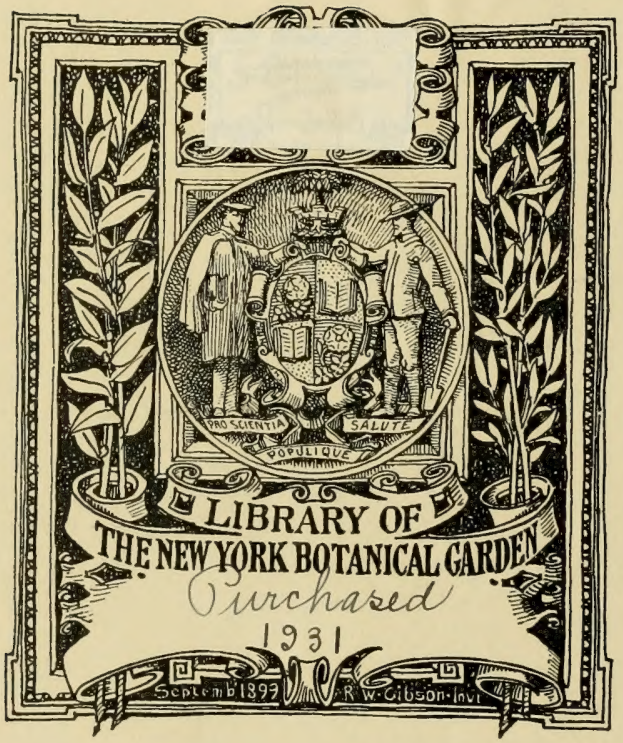







\section{DIE VERBREITUNG}

DER FORSTLICH WICHTIGEN

\section{BÄUME UND STRÄUCHER}

IM UNGARISCHEN STAATE

IM AUPTRAGE DES KÖNIGL. UNG. ACKERBAUMINISTERIUMS,

MIT BENUTZUNG DER UNTER DER LEITUNG DES MINISTERIALRAT, HOCHSCHULPROFESSORS I, P.

LUDWIG FEKETK VORGENOMMENEN

FORSTLICHEN PELANZENGEOGRAPHISCHEN ERHERUNGRN VERFASST

VON

LUDWIG FEKETE UND TIBOR BLATTNY

\section{ZWEITER BAND}

(TABELLEN)

HERAUSGEGEBEN VOM KÖN. UNG. MLISTERIUM FÜR ACKRRBAU

SELMECBANYA

COMMISSIONSVERLAG VON AUGUST JOERGES' WITWE \& SOHN 




\section{DIE VERBREITUNG \\ DER FORSTLICH WICHTIGEN \\ BÄUME UND STRÄUCHER}

IMI UNGARISCHEN STAATE

1 II AUFTRAGE DES KONIGL. UNG. ACKERBAUMINISTERIUMS,

MIT BENLTZUNG DER UNTER DER LEITUNG DES MINISTERIALRAT, HOCHSCHULPROFESORS I. P LUWWG FEKLTE VORGENOMMENEX

FOHSTLICHEN PFLANZEXGEORRAPHISCHEN ERHEHUNGEN VERFASST

VON

LUDWIG FEKETE UND TIBOR BLATTNY

\section{ZIVEITER BANI)}

(TABELLEN)

HERAUSGEGEBEN VOM KÖN. UNG. MINISTERIUM FÜR ACKERBAU

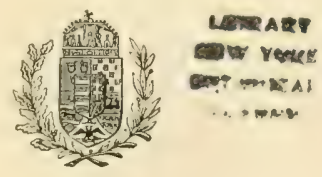

SELMECBÁNYA

COMMISSIONSVERLAG VON AUGUST JOERGES' WITWE \& SOHN 



\section{N H A L'T.}

ERSTER TEIL.

Grenzen der vertikalen Yerbreitung: $\quad$ situ

I. Nordwestkarpathen. . . . . . . . . . . . . . . . 1

II. Zentralkarpathen . . . . . . . . . . . . . . . . . .

III. Nordostkarpathen . . . . . . . . . . . . . . .

IV. Ostkarpathen . . . . . . . . . . . . . . . . 37

V. Sïlkarpathę . . . . . . . . . . . . 4!!

VI. Südungarisches Gebirgsland . . . . . . . . . . . . . 5?

VII. Bihar-Gebirge . . . . . . . . . . . . . . 71

VIII. Ungarisches Mittelgebirge. . . . . . . . . . . . . . $\times 3$

IX. Inselgebirge . . . . . . . . . . . . . . . . . . . . .

X. Hügelland beim Balatonsee . . . . . . . . . . . . . . 91

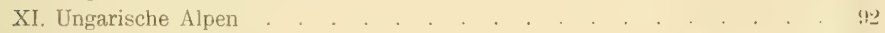

XII. Kroatische Alpen . . . . . . . . . . . . . . . . 41.5

XIII. Siebenbuirger Becken . . . . . . . . . . . . . . . . 1:11

XIV. Großs- und Klein-Alföld (Tiefland) . . . . . . . . . . . . 1:20

ZIVEITER TEIL.

Ühersicht der vertikalen Verbreitungsgrenzen. Mittelwerte fiir das fiebiet des ungarischen staates . . . . . . . . . . . . . 10.2

Register . . . . . . . . . . . . . . . . . 148 



\section{Grenzen der vertikalen Verbreitung. (Nach (iebirgsgegenden.)}

In den Rahmen dieser Tabellen wurden hauptsächlich jene Holzarten aufgenommen, von deren Höhenverbreitung wir genügend barometrische Messungen hatten, um auf Grund derselben die durch die Lage der Fundorte (Abdachung des Gehänges nach) verursachten Höhenunterschiede der Vegetationsgrenzen nach Gebirgsgegenden gruppiert bezeichnen zu können. Diese Arten sind: die allgemein verbreiteten forstlich wichtigen Büume. Hier haben wir auch jene selteneren Arten Aufgenommen, die nur in einzelnen Gegenden zu finden sind, wie Syringa vulgaris, S. Josikaea, Cotinus Coggygria u. A. Der ursprüngliche Arbeitsplan hat sich auf die meisten Sträucher (Ribes-, Cornus-Arten, Viburnum, Lonicera, Cotoneaster u. S. w.) nicht erstreckt; wir behandeln daher diese sowohl, als überhaupt. die hier nicht angeführten Arten in den in diesem Bande befindlichen Tabellen "Übersicht der vertikalen Verbreitungsgrenzen“, eventuell nur an der entsprechenden Stelle des Textteiles (Bd. I.), wobei wir uns auf die Anführung der extremen Grenzdaten (minimum, maximum) beschränken.

Die Anführung der "Summe der Höhengrenzen“ und "Anzahl der Höhengrenzen" informiert uns einesteils über die Zuverlässigkeit der Durchschnitte - Seehöhe in Metern - und bezweckt andererseits die Ergänzung der noch lückenhaften Ergebnisse mit neueren Daten, die Bestïrkung der erreichten Mittelwerte durch Vermehrung der Erhebungen, kurz: sie ermöglicht uns diese Arbeit auf dieser Grundlage fortzusetzen und zu vervollkommnen.

Behufs leichterer Orientierung befindet sich am Schlusse des Bandes ein Register. 



\section{Nordwestkarpathen.}

Höchste Spitze: Babia Góra 1725 m.

\begin{tabular}{|c|c|c|c|c|c|c|c|c|c|c|c|}
\hline$\Xi \Xi$ & $\bar{E}=\overline{\bar{\Sigma}}$ u[ittel. & & & "sla & 1 & i & $r \cdot T \cdot E$ & $1^{m}=$ & $i+1 \cdots 1$ & & InI \\
\hline त्र & $\Xi \exists \equiv$ wert & $N$ & $\mathrm{NO}$ & 0 & $\mathrm{SO}$ & $\mathrm{S}$ & SIV & IV & NW & $\begin{array}{l}\text { Mittel- } \\
\text { wert }\end{array}$ & meinen \\
\hline
\end{tabular}

\section{Picea excelsa Idk.}

i) vereinzelt, untere Grenze

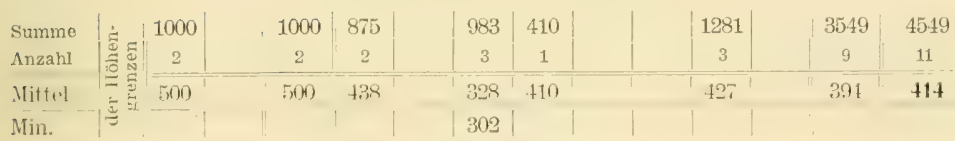

b) rein, oder vorherrschend, untere Grenze

\begin{tabular}{l|c|c|c|c|c|c|c|c|c|c|c|c}
$\begin{array}{l}\text { Sunme } \\
\text { Anzah1 }\end{array}$ & $\begin{array}{l}1800 \\
\text { Nittel }\end{array}$ \\
Min.
\end{tabular}

c) geschlossener Bestand, obere Grenze

\begin{tabular}{|c|c|c|c|c|c|c|c|c|c|c|c|}
\hline summe & & 1237 & 1239 & 1253 & 5398 & 6992 & 10879 & 3930 & $261 \%$ & 30371 & $3161: 3$ \\
\hline Anzahl & $\mid \begin{array}{ll}0 & 5 \\
0 & 0 \\
0 & 0 \\
N\end{array}$ & 1 & 1 & 1 & 4 & 5 & 8 & 3 & 2 & 23 & 24 \\
\hline Mittel & 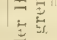 & 1239 & 12351 & 1953 & $135 x$ & 1258 & 1300 & 1312 & 1309 & 1321 & 1317 \\
\hline Max. & & & & & & & 1459 & & & & \\
\hline
\end{tabular}

d) Raumbestand, obere Grenze (IValdgrenze)

\begin{tabular}{|c|c|c|c|c|c|c|c|c|}
\hline Summe & & 3989 & 2831 & 87555 & 7085 & 8597 & 1312 & 32569 \\
\hline Anzahl & 总 & 3 & 2 & 6 & 5 & 6 & 1 & 23 \\
\hline Mitiri & $= \pm$ & 1330 & 1416 & 1.459 & 1417 & $1+33$ & 1312 & $1+16$ \\
\hline Max. & |ี & & & 1506 & & & & \\
\hline
\end{tabular}




\begin{tabular}{|c|c|c|c|c|c|c|c|c|c|c|c|c|}
\hline & & & \multicolumn{9}{|c|}{ Berglehnen nach ihrer Exposition } & \multirow{2}{*}{$\begin{array}{c}\text { Im } \\
\text { Allge- } \\
\text { meinen }\end{array}$} \\
\hline 局邻 & हु) & wort & $\mathrm{N}$ & NO & 0 & SO & $\mathrm{S}$ & SW & W & NIV & $\begin{array}{l}\text { Mittel- } \\
\text { wert }\end{array}$ & \\
\hline
\end{tabular}

e) obere Baumgrenze*

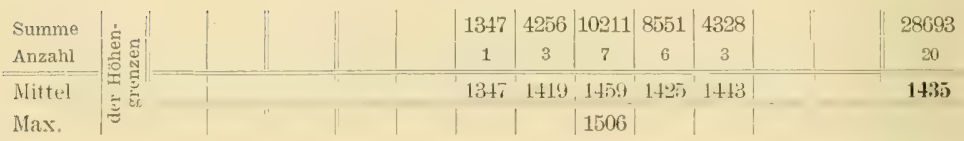

f) verkrüppelt, obere Grenze

$\operatorname{Max}$.

Abies pectimata DC. (A. alba Mill.)

a) vereinzelt, untere Grenze

\begin{tabular}{|c|c|c|c|c|c|c|c|c|}
\hline $\begin{array}{l}\text { Summe } \\
\text { Anzahil }\end{array}$ & 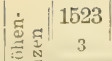 & $\begin{array}{c}1485 \\
3\end{array}$ & $\begin{array}{c}508 \\
1\end{array}$ & $\begin{array}{c}232 \\
1\end{array}$ & $\begin{array}{c}510 \\
1\end{array}$ & $\begin{array}{c}934 \\
3\end{array}$ & $\begin{array}{c}555 \\
1\end{array}$ & $\begin{array}{c}5747 \\
12\end{array}$ \\
\hline Mittel & 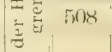 & 495 & 5018 & 232 & 510 & $46 \pi$ & Sisi & 479 \\
\hline
\end{tabular}

b) rein, oder vorherrschend: untere Grenze

\begin{tabular}{|c|c|c|c|c|c|}
\hline Summe & 460 & 1062 & 566 & 1028 & 3116 \\
\hline Anzalıl & 1 & 2 & 1 & 2 & $G$ \\
\hline Mittel & 460 & 531 & 566 & 514 & 519 \\
\hline Min. & & & & 330 & \\
\hline
\end{tabular}

c) vereinzelt (Baumform), obere Grenze

\begin{tabular}{|c|c|c|c|c|c|c|c|c|c|c|c|c|}
\hline Summe & & 3355 & 3355 & 1102 & 1168 & 2366 & 2452 & 6054 & 3566 & 1170 & 17878 & 21233 \\
\hline Anzahl & & 3 & 3 & 1 & 1 & 2 & 2 & 5 & 3 & 1 & 15 & 18 \\
\hline Mittel & & 1118 & 1118 & 1102 & $11 f k$ & 1183 & 1226 & 1211 & $11 \times 9$ & 1170 & 1192 & 1180 \\
\hline Max. & & & & & & & & $|1281|$ & & & & \\
\hline
\end{tabular}

d) verkrüppelt, obere Grenze

Max. $|1300|$

* Bäume mit 8 m. Gipfelhöhe.

** Pax F.' Ptlanzenverbreitung in den Karpathen Bd. II. S. 157. 


\begin{tabular}{|c|c|c|c|c|c|c|c|c|c|c|c|}
\hline \multirow{2}{*}{ 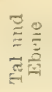 } & \multirow{2}{*}{ 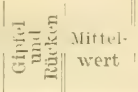 } & \multicolumn{9}{|c|}{ Rerglehnen narh ihrer Exposition } & $\Gamma_{1: 1}$ \\
\hline & & $\mathrm{N}$ & NO & 0 & & $\mathrm{~S}$ & SIV & $\mathrm{IV}$ & $\mathrm{NIV}$ & $\begin{array}{l}\text { Mittel- } \\
\text { wert }\end{array}$ & \\
\hline
\end{tabular}

\section{Pinus silvestris $I_{\text {. }}$.}

a) allgemeine untere Grenze

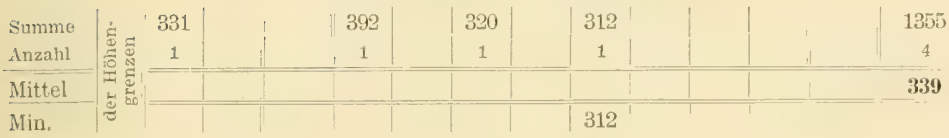

b) rein, oder vorherrschend, obere Grenze

\begin{tabular}{|c|c|c|c|c|c|c|c|c|c|c|}
\hline Summo & $\left|\dot{g}_{-}\right|$ & 1561 & 1561 & 15338 & 639 & 598 & 562 & 568 & 3905 & 5466 \\
\hline AnzahI & ปี & 2 & 2 & 2 & 1 & 1 & 1 & 1 & 6 & 8 \\
\hline Nittel & & is1 & $7 \times 1$ & 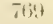 & fis! & $5: K$ & 5 (it) & $5 x+s$ & (ii) & (i) \\
\hline
\end{tabular}

Max. $\left.\right|^{\circ} \quad 1829$

c) vereinzelt, obere Grenze

\begin{tabular}{|c|c|c|c|c|c|c|c|c|c|}
\hline Summe & & 950 & 3495 & 4445 & 1860 & 747 & 923 & 3530 & 7975 \\
\hline Anzahl & $\stackrel{5}{0}$ & 1 & 4 & 5 & 2 & 1 & 1 & 4 & 9 \\
\hline Mittel & & 9.50 & 8.4 & xxy & (13) & $7 t 7$ & 123 & 883 & 886 \\
\hline Max. & & 950 & & & & & & & \\
\hline
\end{tabular}

\section{Pinus montana Mill.}

a) untere Grenze

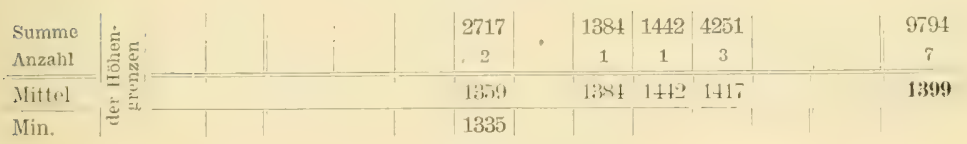

b) obere Grenze

Max.

\section{Juniperus nana Willd.}

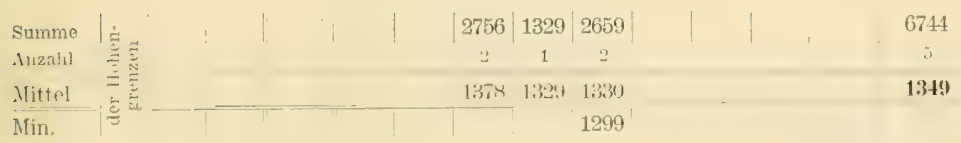

b) obere Grenze

(keine) 


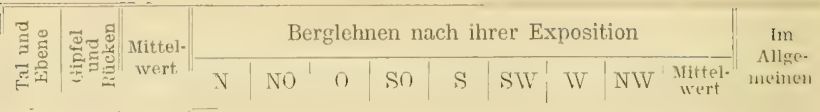

Fagus silvatica $\mathbf{L}$.

a) vereinzelt, untere Grenze

\begin{tabular}{|c|c|c|c|c|c|c|c|c|c|c|c|c|}
\hline Summe & 1282 & 1282 & 1680 & 340 & 515 & 248 & 2137 & 340 & 1968 & 315 & 7543 & 889 \\
\hline Anzil & 4 & + & 3 & 1 & 2 & 1 & i) & 1 & 5 & 1 & 22 & 34 \\
\hline Mittel & 321 & $3 \geq 1$ & 336 & 310 & 30.4 & 214 & 356 & $3+11$ & 391 & $31 \pi$ & $3+3$ & 339 \\
\hline Min. & 200 & & & & & & & & & & & \\
\hline
\end{tabular}

b) bestandbildend, untere Grenze

\begin{tabular}{|c|c|c|c|c|c|c|c|c|c|c|c|c|c|c|}
\hline Summe & \multirow{4}{*}{ 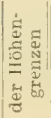 } & 1223 & 525 & 1747 & 2621 & 1472 & 1077 & 1565 & 1973 & 1598 & 907 & 268 & 11481 & 13228 \\
\hline Anzahl & & 4 & 1 & 5 & 8 & 4 & 3 & 4 & 4 & 4 & 2 & 1 & 30 & 35 \\
\hline Mittel & & 300 & 525 & $3+9$ & 328 & 368 & 354 & 391 & 493 & 399 & tot & 268 & 383 & 378 \\
\hline Min. & & & & & 222 & & & & & & & & & \\
\hline
\end{tabular}

c) bestandbildend, obere Grenze

\begin{tabular}{l|c|c|c|c|c|c}
$\begin{array}{l}\text { Summe } \\
\text { Anzahl }\end{array}$ \\
\hline Mittel \\
\hline Max.
\end{tabular}

d) veremzelt (als Mischholz), obere Grenze

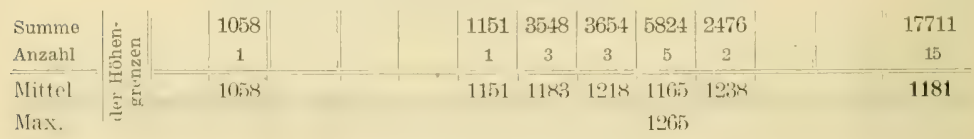

e) Strauchform, obere (irenze

\begin{tabular}{|c|c|c|c|c|c|c|}
\hline $\begin{array}{l}\text { Summe } \\
\text { Anzahl }\end{array}$ & $\begin{array}{c}1226 \\
1\end{array}$ & & $\begin{array}{c}2404 \\
2\end{array}$ & $\begin{array}{c}2448 \\
2\end{array}$ & $\begin{array}{c}2493 \\
2\end{array}$ & $\begin{array}{c}9761 \\
8\end{array}$ \\
\hline Mittel & 1396 & 1190 & 1202 & 1224 & 1217 & 1920 \\
\hline
\end{tabular}

\section{Quercus sessiliflora Salisb.}

a) rein, oder vorherrschend, obere Grenze

\begin{tabular}{|c|c|c|c|c|c|c|c|}
\hline Summe & 541 & 504 & 1173 & 1694 & 453 & 488 & 4853 \\
\hline Anzahl & 1 & 1 & 2 & 3 & 1 & 1 & 9 \\
\hline Mittel & 541 & 504 & 58 & 565 & 453 & 488 & 539 \\
\hline Max. & & & & 646 & & & \\
\hline
\end{tabular}




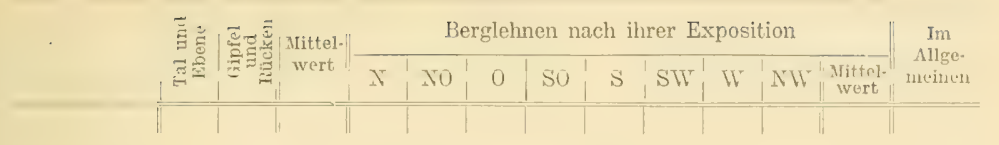

b) rereiuzelt (Baunform), obere Grenze

\begin{tabular}{|c|c|c|c|c|c|c|c|c|c|c|}
\hline Summe & & 2772 & 2068 & $\mid 1964$ & 5.18 & 2047 & 2029 & 2181 & 712 & 14321 \\
\hline Anzahl & กำ & 4 & 3 & 3 & 1 & 3 & 3 & 3 & 1 & 21 \\
\hline Mittel & 5 & 693 & tist & (fin & 518 & $6 x$ & 676 & 72. & 712 & $6 \times 2$ \\
\hline Max. & & & 802 & & & & & & & \\
\hline
\end{tabular}

c) verkruippelt

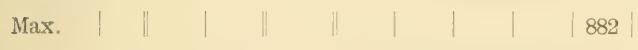

Quercus perlunculata Ehrh. (qu. Robur L.)

vereinzelt obere Grenze

\begin{tabular}{|c|c|c|c|c|c|c|c|c|c|c|c|}
\hline Summe & 4075 & 996 & 5071 & 2774 & 401 & 509 & 1279 & 1730 & 938 & 7641 & 12702 \\
\hline Auzahl & 9 & 2 & 11 & 6 & 1 & 1 & 3 & 4 & 2 & 17 & $i x$ \\
\hline Mittel & 453 & 498 & $4 t i 1$ & $H+2$ & 41)1 & 511:? & +24 & +33 & t6i9 & H9 & 45.3 \\
\hline Max. & & & & 618 & & & & & & & \\
\hline
\end{tabular}

\section{Carpinus Betulus L.}

a) rein, oder vorherrschend, obere Grenze

\begin{tabular}{|c|c|c|c|c|c|c|c|c|c|c|c|c|c|}
\hline Summe & & 427 & 918 & 1345 & 2644 & 357 & 339 & 3363 & 2265 & 310 & 370 & $96+8$ & 10993 \\
\hline Anzahl & & 1 & 2 & 3 & 6 & 1 & 1 & 8 & 5 & 1 & 1 & 23 & 26 \\
\hline $\begin{array}{l}\text { Mittel } \\
\text { Max. }\end{array}$ & 严。 & 427 & 454 & $+t x$ & 411 & (in) & ;i3! & $\begin{array}{l}4-31 \\
562\end{array}$ & tiis & 3111 & :iul & +19 & +2:3 \\
\hline
\end{tabular}

b) vereinzelt, obere Grenze

\begin{tabular}{|c|c|c|c|c|c|c|c|c|c|c|c|}
\hline Summe & & 643 & 762 & $1+05$ & 509 & 707 & 5067 & 988 & 747 & 8018 & $9 \pm 23$ \\
\hline Anzahl & $\frac{2}{0} \bar{E}$ & 1 & 1 & 2 & 1 & 1 & 8 & 2 & 1 & 1.3 & 15 \\
\hline Mittul & $=\frac{7}{5}$ & tit3 & $76+3$ & 708 & $\pi 09$ & 7118 & 53,3 & $4 !+1$ & 717 & $6 i 1$. & 628 \\
\hline Max. & 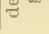 & & & & & & 867 & & & & \\
\hline
\end{tabular}

\section{Alnus glutinosa Gärtı.}

obere Grenze

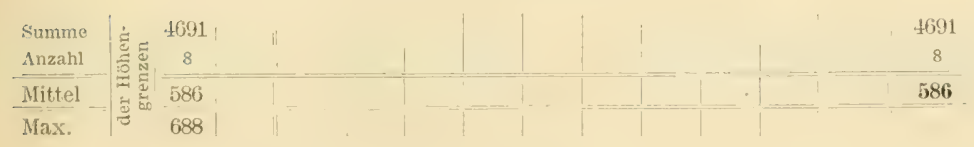




\begin{tabular}{|c|c|c|c|c|c|c|c|c|c|c|c|c|}
\hline \multirow{2}{*}{ 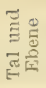 } & \multirow{2}{*}{ 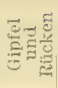 } & \multirow{2}{*}{$\begin{array}{l}\text { Mittel- } \\
\text { wert }\end{array}$} & \multicolumn{9}{|c|}{ Berglehnen nach ihrer Exposition } & \multirow{2}{*}{$\begin{array}{l}\operatorname{Im}_{\text {Allge }} \\
\text { meinen }\end{array}$} \\
\hline & & & $\mathrm{N}$ & NO & 0 & & $\mathrm{~S}$ & & & NIV & $\begin{array}{l}\text { Mittel- } \\
\text { wert }\end{array}$ & \\
\hline
\end{tabular}

\section{Alnus incana Willd.}

obere Grenze

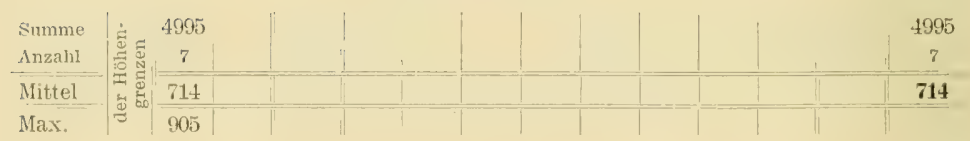

Populus tremula $\mathrm{L}$.

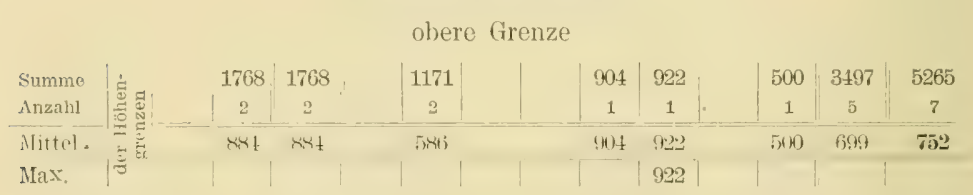

\section{Ulmus montana With. (U. scabra Hill.)}

a) untere Grenze

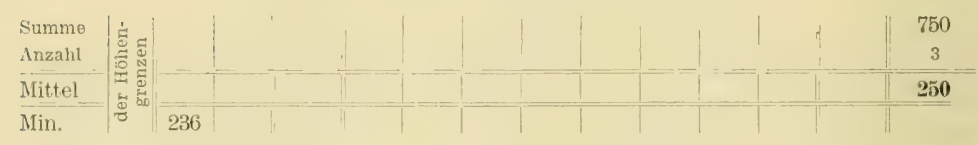

b) obere Grenze

\begin{tabular}{|c|c|c|c|c|c|c|c|c|c|c|}
\hline Summe & & 714 & 2185 & 2899 & 1427 & 808 & 962 & 670 & 3867 & 6766 \\
\hline Anzahl & 몽 & 1 & 3 & 4 & 2 & 1 & 1 & 1 & 5 & 9 \\
\hline Mittel & 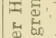 & 714 & 728 & 725 & 714 & 808 & 963 & 670 & 773 & 752 \\
\hline Max. & & & & & & & 962 & & & \\
\hline
\end{tabular}

\section{Acer platanoides $\mathbf{L}$.}

Baumform, obere Grenze

\begin{tabular}{|c|c|c|c|c|c|c|}
\hline Summe & 806 & 829 & 878 & 1628 & 779 & 4920 \\
\hline Anzahl & 1 & 1 & 1 & 2 & 1 & 6 \\
\hline Mittel & xilfi & $8 \% 9$ & $x \cdot x$ & 81. & $7 \pi 9$ & 820 \\
\hline Max. & & & 878 & & & \\
\hline
\end{tabular}




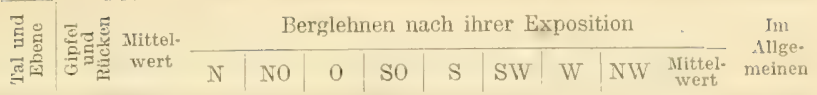

Acer Pseudoplatamus $L$.

Baumform, obere Grenze

\begin{tabular}{|c|c|c|c|c|c|}
\hline Summe & \multirow{4}{*}{ 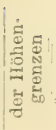 } & 1168 & 1223 & 1058 & 3449 \\
\hline Anzahl & & 1 & 1 & 1 & 3 \\
\hline Mittel & & 1168 & 1223 & 1058 & 1150 \\
\hline Max. & & & 1223 & & \\
\hline
\end{tabular}

\section{Acer campestre $\mathbf{I}$.}

a) Baumform, obere Grenze

\begin{tabular}{|c|c|c|c|c|c|c|c|c|c|}
\hline Summe & $\dot{E}$ & 1053 & 740 & 1793 & 430 & 380 & 1270 & 2080 & 3873 \\
\hline Anzahl & 起 & 3 & 1 & 4 & 1 & 1 & 2 & 4 & 8 \\
\hline Mittel & $=\bar{D}$ & 3501 & 740 & 448 & 430 & 380 & 635 & 520 & 484 \\
\hline Iax. & & & $7+0$ & & & & & & \\
\hline
\end{tabular}

b) Strauchform, obere Grenze

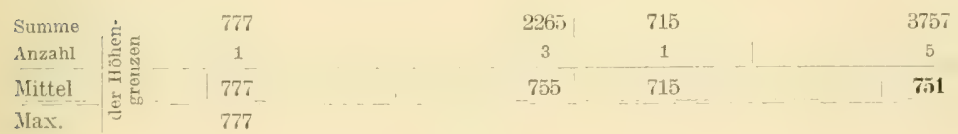

\section{Fraximus excelsior $\mathbf{I}$.}

Baumform, obere Grenze

\begin{tabular}{|c|c|c|c|c|c|c|c|c|c|c|c|c|}
\hline Summe & '. & 3456 & 2572 & 6028 & 789 & 1348 & 1768 & 1671 & 962 & 780 & 7318 & 13346 \\
\hline Inzahl & 号怘 & 5 & 3 & 8 & 1 & 2 & 2 & 2 & 1 & 1 & $?$ & 17 \\
\hline Mittel & 可 & 691 & 857 & $75 \pm$ & 789 & 674 & 884 & 836 & 962 & 780 & 813 & 785 \\
\hline Іах. & & & & & & & & 980 & & & & \\
\hline
\end{tabular}

\section{Sorbus ancupariat L.}

a) Baumform, obere Grenze

\begin{tabular}{l|c|c|c|} 
Summe \\
Anzahl \\
Mittel
\end{tabular}




\begin{tabular}{|c|c|c|c|c|c|c|c|c|c|c|c|c|}
\hline \multirow{2}{*}{ 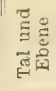 } & \multirow{2}{*}{ 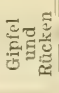 } & \multirow{2}{*}{ | } & \multicolumn{9}{|c|}{ Berglehnen nach ihrer Exposition } & \multirow{2}{*}{$\begin{array}{c}\text { Im } \\
\text { Allge- } \\
\text { meinen }\end{array}$} \\
\hline & & & $\mathrm{N}$ & NO & 0 & SO & S & SW & W & NW & $\begin{array}{l}\text { Mittel- } \\
\text { wert }\end{array}$ & \\
\hline
\end{tabular}

b) Strauchform, obere Grenze

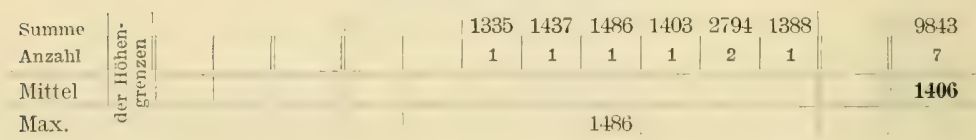

Crataegus monogyua Jacq. und oxyacantha $\mathbf{L}$.

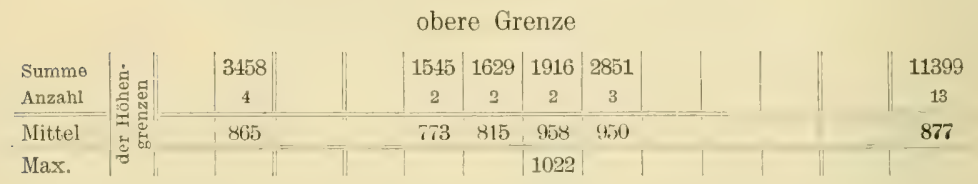




\section{Centralkarpathen.}

Magas-Tátra = Hohe Tátra (Ferenc József-Spitze) 2663 m., Alacsony-Tátra = Niedere Tátra (Gyömbér) 2045 m., Nagy-Fátra = Grosse Fátra (Osztredok) 1591 m., Kriván-Fátra (Koncsita-Kriván) 1711 m., Kis-Fátra = Kleine Fátra 1477 m., Polana 1459 m., Szepes-Gömöri Érchegység $=$ Szepes-Gömörer Erzgebirge (Stolica) $1480 \mathrm{~m}$.

\begin{tabular}{|c|c|c|c|c|c|c|c|c|c|c|c|}
\hline 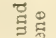 & 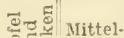 & \multicolumn{9}{|c|}{ Berglehnen nach ihrer Exposition } & Im \\
\hline 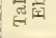 & $\mathrm{o}^{5}$ wert & $\mathrm{N}$ & No & 0 & so & $\mathrm{S}$ & SW & W & N & Nittel- & meinen \\
\hline
\end{tabular}

\section{Picea excelsa Lk.}

a) vereinzelt, untere Grenze

\begin{tabular}{|c|c|c|c|c|c|c|c|c|c|c|c|c|c|c|}
\hline Summe & & 5487 & 769 & 6256 & 5317 & 1506 & 4167 & 1957 & 2599 & $\mid 1922$ & | 2340 & | 2039 & $217 \pm 7$ & 28003 \\
\hline Anzah & 옹 & 11 & 1 & 12 & 11 & 3 & 9 & 3 & 4 & 4 & 4 & 4 & 42 & 54 \\
\hline & चु & 499 & 769 & 521 & 483 & 503 & 463 & 652 & 650) & $4 \% L$ & $5+i 0$ & 510 & $51 \%$ & 519 \\
\hline Min. & & & & & & & & & & 302 & & & & \\
\hline
\end{tabular}

b) rein oder vorherrschend, untere Grenze

Summe |

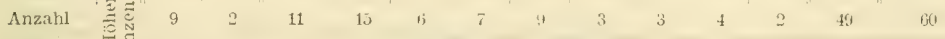

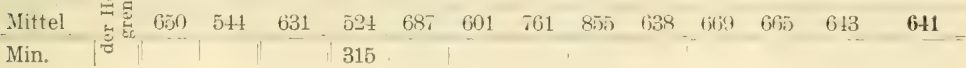

c) geschlossener Bestand, obere Grenze

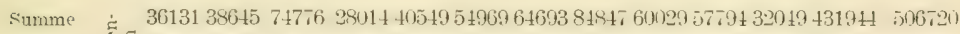

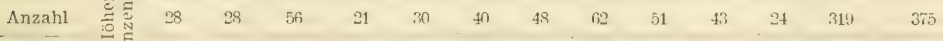

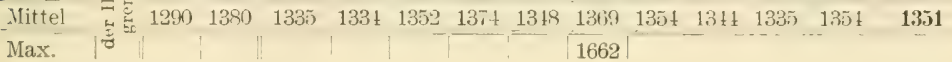

d) Raumbestand, obere Grenze (Waldgrenze)

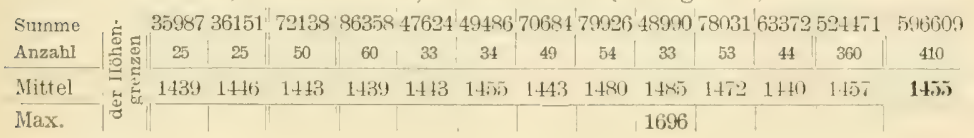




\begin{tabular}{|c|c|c|c|c|c|c|c|c|c|c|c|}
\hline \multirow{2}{*}{\multicolumn{2}{|c|}{ 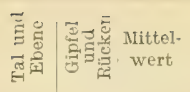 }} & \multicolumn{9}{|c|}{ Berglehnen nach ihrer Exposition } & \multirow{2}{*}{$\begin{array}{l}\text { In } \\
\text { Allge- } \\
\text { meinen }\end{array}$} \\
\hline & & $\mathrm{N}$ & NO & 0 & $\mathrm{SO}$ & $\mathrm{S}$ & SW & W & $\mathrm{NW}$ & $\begin{array}{l}\text { Mittel- } \\
\text { wert }\end{array}$ & \\
\hline
\end{tabular}

e) obere Baumgrenze*

Summe | \& $2918944062732517432850071813159127811637181119,10890954759658240,731491$

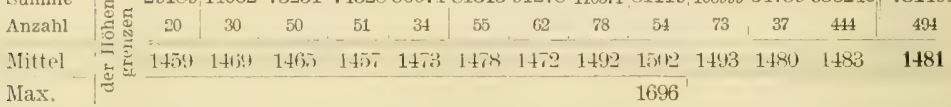

f) Raumbestand mit verkruppelten Fichten, obere Grenze

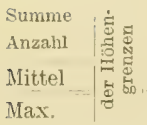

1744

g) verkrïppelt, obere Grenze

\begin{tabular}{|c|c|c|c|c|c|c|c|c|c|c|c|}
\hline Summe & & 1762 & 1860 & 1632 & 1770 & $357+4$ & 5535 & 5350 & 3889 & 2017 & 27389 \\
\hline Anzahl & : & 1 & 1 & 1 & 1 & 2 & 3 & 3 & 2 & 1 & 15 \\
\hline Mittel & $=5$ & $17 t i 2$ & 1860 & 1632 & 1770 & 1787 & 1815 & 1783 & $194 \pi$ & 3017 & 1896 \\
\hline Max. & $\tilde{\tau}$ & & & & & & & & 2057 & & \\
\hline
\end{tabular}

\section{Abies pectinata 1)C. (A. alba Hill.)}

a) vereinzelt, untere Grenze

\begin{tabular}{|c|c|c|c|c|c|c|c|c|c|c|c|c|c|}
\hline Summe & $\dot{c}$ & 3472 & 1137 & 4609 & 2509 & 1789 & 655 & 610 & $95 \check{~}$ & 1703 & 1731 & 9982 & 14591 \\
\hline Anzahi & : & 8 & 2 & 10 & 7 & 5 & 2 & 2 & 2 & 3 & 5 & 26 & 36 \\
\hline Mittel & 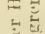 & 431 & $56 t^{3}$ & tiil & $35 x$ & $35 \mathrm{~K}$ & 328 & $3: 11$ & 478 & 568 & $3+6$ & 384 & 405 \\
\hline Min. & & & & & & & 215 & & & & & & \\
\hline
\end{tabular}

b) rein oder vorherrschend, untere Grenze

\begin{tabular}{|c|c|c|c|c|c|c|c|c|c|c|c|c|c|c|}
\hline Sumne & & 2058 & 1172 & 3230 & 3777 & \pm 101 & 2374 & 1940 & 1560 & 914 & 2761 & 953 & 18380 & 21610 \\
\hline Anzahl & & 5 & 2 & 7 & 9 & 10 & 5 & 4 & 3 & 2 & 5 & 2 & 40 & 47 \\
\hline Mittel & $=5$ & 412 & Exti & 461 & 120 & 4111 & $+7 i$ & 485 & $504)$ & tin & $5 \tilde{2} 2$ & 177 & 460 & +60 \\
\hline Iin. & & & & & & & 215 & & & & & & & \\
\hline
\end{tabular}

c) rein oder vorherrschend, obere Grenze

\begin{tabular}{|c|c|c|c|c|c|c|c|c|c|c|c|c|c|c|}
\hline Sixmme & & 2023 & 15635 & 17658 & 12640 & 6980 & $15+15$ & 13384 & 13251 & 8860 &, 14232 & 20381 & $105 \tilde{143}$ & 122801 \\
\hline Anzahl & 1 : & 2 & 15 & 17 & 12 & 7 & 14 & 12 & 12 & 8 & 13 & 19 & 97 & 114 \\
\hline Miltal & 武昰 & 1012 & 1042 & $1613: 1$ & $111 \pi)^{2}$, & $94 \%$ & 11011 & 1115 & $110 t$ & $110 \pi$ & $109 \bar{i}$ & 10.3 & $10 \times 1$ & 1077 \\
\hline ax. & & & & & & & & 1335 & & & & & & \\
\hline
\end{tabular}

* Bäume nit 8 m. Gipfelhöhe. 


\begin{tabular}{|c|c|c|c|c|c|c|c|c|c|c|c|c|}
\hline \multirow{2}{*}{ 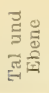 } & \multirow{2}{*}{ 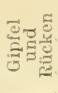 } & \multirow{2}{*}{$\begin{array}{c}\text { Mittel- } \\
\text { wert }\end{array}$} & \multicolumn{9}{|c|}{ Berglehnen nach ihrer Exposition } & \multirow{2}{*}{$\begin{array}{c}\text { Im } \\
\text { Allge- } \\
\text { meinen }\end{array}$} \\
\hline & & & $N$ & No & 0 & SO & $\mathrm{S}$ & SWV & WV & NIV & $\begin{array}{l}\text { Mittel- } \\
\text { wert }\end{array}$ & \\
\hline
\end{tabular}

d) vereinzelt (Baumform), obere Grenze

\begin{tabular}{|c|c|c|c|c|c|c|c|c|c|c|c|c|c|c|}
\hline summe & & $565 t$ & 19068 & 5253 & 2 & 00007 & 4126 & D1228 & 71044 & 37199 & 25629 & 28009 & 305546 & 330778 \\
\hline Anzahl & & 5 & 15 & 20 & 18 & 25 & 35 & 40 & 56 & 30 & 20 & 18 & 242 & 262 \\
\hline $\begin{array}{l}\text { littel } \\
\text { Max. }\end{array}$ & 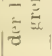 & 1111 & 131:2 & 1240 & $1: 31$ & 1223 & 1261 & 1281 & $\begin{array}{l}127 ? \\
1562\end{array}$ & $12+11$ & $1: 2 \times 1$ & $\begin{array}{l}1278 \\
1562\end{array}$ & 12433 & \\
\hline
\end{tabular}

e) verkrüppelt, obere Grenze

\begin{tabular}{|c|c|c|c|c|c|c|c|c|c|c|c|c|}
\hline s'munk & $x: 45$ & x.45 & 9612 & 5) 221 & $16+59$ & $2+817$ & 19724 & 9382 & $1252 t$ & 5294 & 103037 & $1112 \times 3$ \\
\hline Anzaht & 6 & 6 & 7 & 4 & 12 & 18 & 14 & 7 & 9 & 4 & 75 & 81 \\
\hline Mitt.l & $13 \pi t$ & $137 t$ & 1373 & $130 \tilde{5}$ & 1372 & 1379 & $1+0: 3$ & 1341) & 1342 & 1325 & 1374 & 1374 \\
\hline $\operatorname{Max}$. & 1600 & & & & & & & & & & & \\
\hline
\end{tabular}

\section{Pinus silvestris $\mathbf{L}$.}

a) untere Grenze

\begin{tabular}{|c|c|c|c|c|c|c|c|c|c|}
\hline Summe & 1433 & 1440 & 440 & 465 & 930 & 973 & 1281 & 480 & $7+12$ \\
\hline Anzall1 & 造 & 3 & 1 & 1 & 2 & 2 & 4 & 1 & 18 \\
\hline Mittel & $=\sin$ & $4(x)$ & +10 & 465 & $4(6 \pi)$ & 487 & 3211 & (ini) & +13 \\
\hline Min. & $1 \% 232$ & & & & & & & & \\
\hline
\end{tabular}

b) rein, oder vorherrschend, obere Grenze

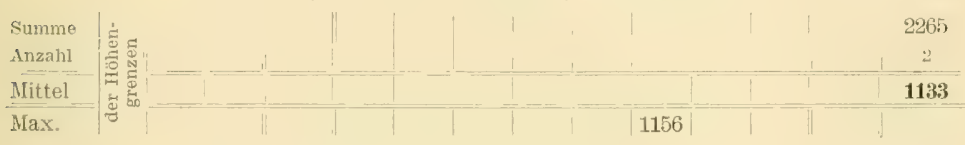

c) vereinzelt (Baumform), obere Grenze

\begin{tabular}{|c|c|c|c|c|c|c|c|c|c|c|}
\hline Summe & 2305 & 1340 & 1042 & 1100 & 7617 & 7621 & 2498 & 4782 & 4761 & 33066 \\
\hline Anzahl & 동 & 1 & 1 & 1 & 6 & 6 & 2 & 4 & 4 & 27 \\
\hline . Iittel & 1103 & 1310 & 1012 & 1100 & 1270 & $12-1$ & 1219 & 1196 & 1190 & 1225 \\
\hline Max. & $1^{2}: 1$ & & & & & 1465 & & & & \\
\hline
\end{tabular}

d) verkrüppelt, obere Grenze

\begin{tabular}{|c|c|c|c|c|c|c|c|}
\hline Summe & 1358 & 1406 & 6858 & 1450 & 1362 & 1350 & 13781 \\
\hline Anzahl & 1 & 1 & 5 & 1 & 1 & 1 & 10 \\
\hline $\begin{array}{l}\text { MittıI } \\
\text { Max.* }\end{array}$ & $13 \% 8$ & $14 n 6$ & $13 \% 2$ & $1+50$ & 1362 & 1350 & 1378 \\
\hline
\end{tabular}

* Kleiner, als das maximum der ob. Grenze der Baumform. 


\begin{tabular}{|c|c|c|c|c|c|c|c|c|c|c|c|c|}
\hline \multirow{2}{*}{ 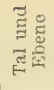 } & \multirow{2}{*}{ 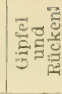 } & \multirow{2}{*}{$\begin{array}{c}\text { Mittel. } \\
\text { wert }\end{array}$} & \multicolumn{9}{|c|}{ Berglehnen nach ihrer Exposition } & \multirow{2}{*}{$\begin{array}{c}\text { Im } \\
\text { Allge- } \\
\text { meinen }\end{array}$} \\
\hline & & & $\mathrm{N}$ & NO & 0 & SO & $\mathrm{S}$ & SW & W & NIV & $\begin{array}{c}\text { Mittel- } \\
\text { wert }\end{array}$ & \\
\hline
\end{tabular}

\section{Pinus Cembra I.,}

a) untere Grenze

\begin{tabular}{|c|c|c|c|c|c|c|c|c|c|c|c|c|c|}
\hline Sismme & & 3454 & 3451 & 4154 & 5752 & 6929 & 4130 & 3094 & 1538 & 5๊ 15 & 8048 & 39190 & 42641 \\
\hline Anzahl & & 3 & 3 & 3 & 4 & 5 & 3 & 2 & 1 & 4 & 6 & 28 & 31 \\
\hline Iittel & 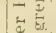 & $115 \mathrm{~L}$ & 1151 & $13 \% 5$ & 1438 & $13 \times 6$ & 1377 & $15+7$ & 1538 & $13 \times t j$ & $13+1$ & $1400)$ & 1376 \\
\hline Min. & & 1021 & & & & & & & & & & & \\
\hline
\end{tabular}

b) Baumform, obere Grenze

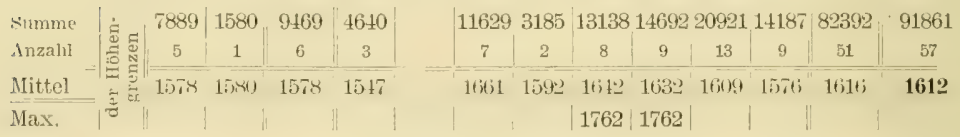

c) verkriippeit, obere Grenze

\begin{tabular}{|c|c|c|c|c|c|c|c|c|c|c|c|c|}
\hline Sinmme & & 1611 & 3212 & 4823 & 3257 & 3420 & 5283 & 1821 & 5123 & 10171 & 29075 & 33898 \\
\hline Anzahl & & 1 & 2 & 3 & 2 & 2 & 3 & 1 & 3 & 6 & 17 & 20 \\
\hline Mittel & & 1611 & 16060 & $16 \mathrm{~km}$ & 1628 & 1710 & $180 t 1$ & 1821 & $170 \mathrm{~K}$ & $165 \%$ & 1710 & 1695 \\
\hline Max. & & & & & & & & 1821 & & & & \\
\hline
\end{tabular}

\section{Pinus montana Mill.}

a) sporadisch, untere Grenze

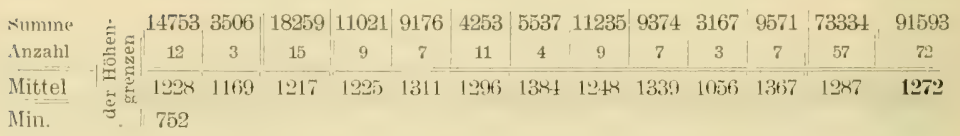

b) massenhaft, untere" Grenze

\begin{tabular}{|c|c|c|c|c|c|c|c|c|c|c|c|c|c|c|}
\hline Sitmme & & 7040 & 1481 & 8521 & 7238 & 9940 & 4575 & 7314 & 5918 & 5579 & 8143 & 6601 & 55638 & 64159 \\
\hline Anzah! & ํㅗㅇ & 5 & 1 & 6 & 5 & 7 & 3 & 5 & 4 & 4 & 6 & 5 & 39 & 45 \\
\hline ittel & & $1+1 \mathrm{~s}$ & $1|x|$ & $1+241$ & $14 k$ & 1490 & $15 \div 5$ & 1469 & $1.1 \times 0$ & $134 \%$ & 1407 & 13291 & 1427 & 1426 \\
\hline in. & & & & & & & & & & & & 980 & & \\
\hline
\end{tabular}

* Kommit nur in der Hohen Tátra vor. 


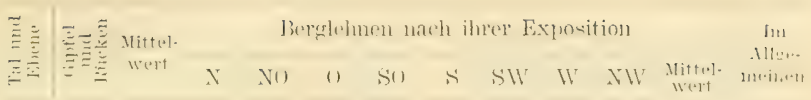

c) massenhaft, obere Grenze

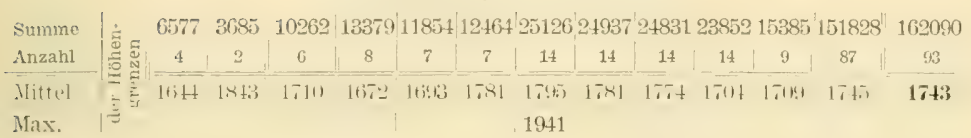

d) sporadisch, obere Grenze

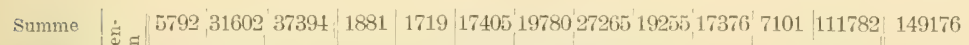

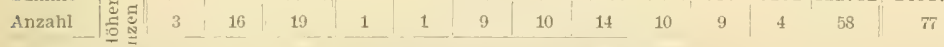

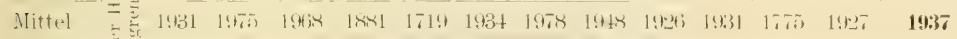
Max. 2140

\section{Larix europaea DC. (L. decidua Mill.)}

a) untere Grenze

\begin{tabular}{|c|c|c|c|c|c|c|c|c|c|}
\hline Summe & $\Leftrightarrow \quad 680$ & 435 & 650 & 680 & $30+6$ & 680 & 1153 & 2370 & 9694 \\
\hline Anzahl & $\frac{D}{3} \sqrt{\pi} \quad 1$ & 1 & 1 & 1 & 4 & 1 & 2 & 4 & 15 \\
\hline Mittel & $=\frac{2}{3}$ en & 43\% & 6กัก & 680 & 762 & 680 & $5 \pi$ & 598 & 646 \\
\hline Miı. & & & & & & & 398 & & \\
\hline
\end{tabular}

b) rein, oder vorherrschend, obere Grenze

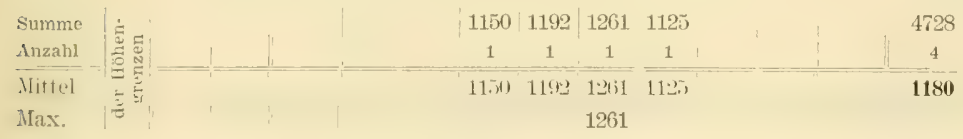

c) Raumbestand, obere Grenze*

\begin{tabular}{l|l|c|c|c|c|c}
$\begin{array}{l}\text { Summe } \\
\text { Anzahl }\end{array}$ \\
\hline $\begin{array}{l}\text { Yittel } \\
\text { Max. }\end{array}$ \\
Max.
\end{tabular}

d) vereinzelt (Baumform), obere Grenze

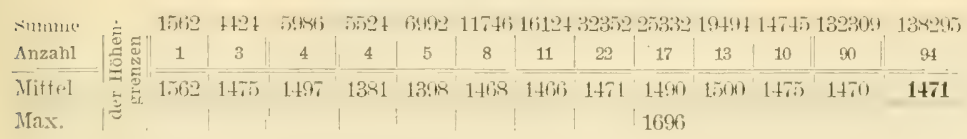

* Bezieht sich nur auf die Hohe Tátra. 


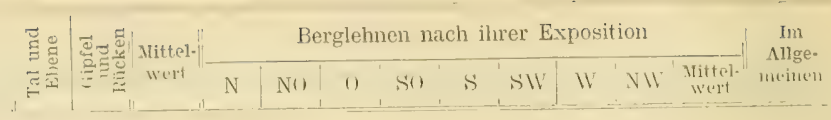

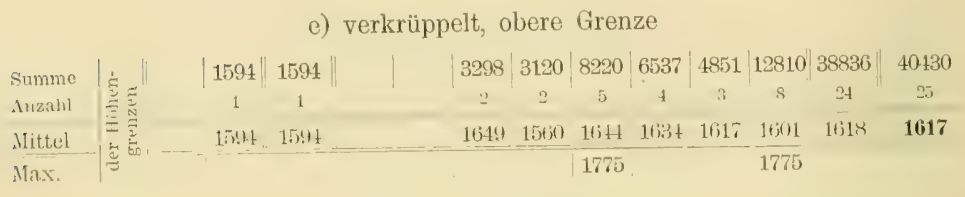

\section{'Taxus bacenta I.}

a) untere Grenze

\begin{tabular}{|c|c|c|c|c|c|c|c|}
\hline Summe & 3820 & 1098 & 760 & 1536 & 1516 & 8730 & 8730 \\
\hline Anzalil & G & 2 & 1 & 2 & & 13 & 13 \\
\hline Mittel & 637 & 549 & 760 & 768 & 758 & 672 & 672 \\
\hline Min. & & 426 & & & & & \\
\hline
\end{tabular}

b) obere Grenze

\begin{tabular}{|c|c|c|c|c|c|c|c|c|c|c|}
\hline $\begin{array}{l}\text { Summe } \\
\text { Anzahl }\end{array}$ & 동 & $\begin{array}{c}3166 \\
3\end{array}$ & $\begin{array}{c}1800 \\
2\end{array}$ & $\begin{array}{c}950 \\
1\end{array}$ & $\begin{array}{c}962 \\
1\end{array}$ & $\begin{array}{c}2949 \\
3\end{array}$ & $\begin{array}{c}850 \\
1\end{array}$ & $\begin{array}{c}4048 \\
4\end{array}$ & $\begin{array}{c}3193 \\
3\end{array}$ & $\begin{array}{c}17918 \\
18\end{array}$ \\
\hline Mittel & 揟 & 1055 & 900 & 950 & 9132 & 983 & 850 & 1012 & 1064 & 995 \\
\hline Max. & & & $\|$ & & & & & & 1300 & \\
\hline
\end{tabular}

\section{Juniperus communis L.}

\begin{tabular}{|c|c|c|c|c|c|c|c|c|c|c|c|c|}
\hline $\begin{array}{l}\text { summe } \\
\text { Anzalne }\end{array}$ & 11565 & $\begin{array}{c}11565 \\
?\end{array}$ & $\begin{array}{c}2581 \\
2\end{array}$ & $\left|\begin{array}{c}3569 \\
3\end{array}\right|$ & $\begin{array}{c}3744 \\
3\end{array}$ & $\begin{array}{c}4928 \\
4\end{array}$ & $\begin{array}{c}3889 \\
3\end{array} \mid$ & $\begin{array}{c}1358 \\
1\end{array}$ & $\begin{array}{c}1139 \\
1\end{array}$ & $\begin{array}{c}1197 \\
1\end{array}$ & $\begin{array}{c}22405 \\
18\end{array}$ & $\begin{array}{c}33970 \\
27\end{array}$ \\
\hline Mittel & 1.285 & $128 \pi$ & 1391 & 1190. & $12+8$ & 1232 & 1296 & 1358 & 1139 & 1197 & $124 \%$ & 1258 \\
\hline Max. & 1405 & & & & & & & & & & & \\
\hline
\end{tabular}

\section{Juniperus nana Willl.}

a) untere Grenze

\begin{tabular}{|c|c|c|c|c|c|c|c|c|c|c|c|c|c|}
\hline sйmme & & 1221 & 4909 & 9130 & 2812 & 1417 & 2913 & 6170 & +830 & 1491 & 1140 & 21103 & 30233 \\
\hline Auzahı & & 3 & 4 & 7 & 2 & 1 & 2 & 5 & 4 & 1 & 1 & $16 i$ & 23 \\
\hline Mittel & है है & 1407 & 1227 & 1304 & 1406 & $1+17$ & $14 \tilde{7}$ & 1294 & 1308 & 1491 & 1140 & 1319 & 1314 \\
\hline Min. & & & & & & & & & 1090 & & & & \\
\hline
\end{tabular}




Berglehnen nach ihrer Exposition
|

b) obere Grenze

\begin{tabular}{|c|c|c|c|c|c|c|c|c|c|c|c|}
\hline Summe & & 4149 & 4149 & 2002 & 2002 & 11946 & 10187 & 5782 & 2000 & 33919 & 38068 \\
\hline Anzahl & 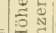 & 2 & 2 & 1 & 1 & 6 & 5 & 3 & 1 & 17 & 19 \\
\hline Mittrl & $\begin{array}{l}5 \\
5\end{array}$ & 301, & $315 \pi$ & $30 \mathrm{H}$ & 3012 & $19: 21$ & 318 & 1937 & 3040 & 1445 & 2004 \\
\hline Мах. & $1=$ & & & & & 2140 & & & & & \\
\hline
\end{tabular}

\section{Fagus silvatica $\mathbf{L}$.}

a) vereinzelt, untere Grenze

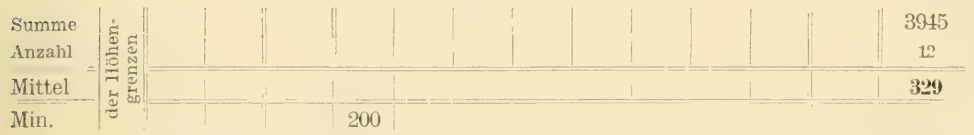

b) bestandbildend, untere Grenze

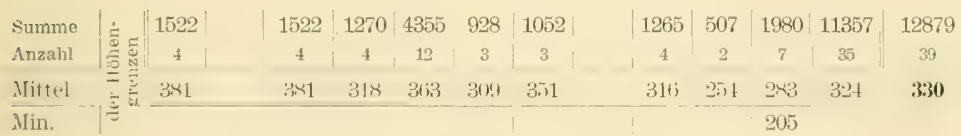

c) Bestandbildung im Allgemeinen, obere Grenze

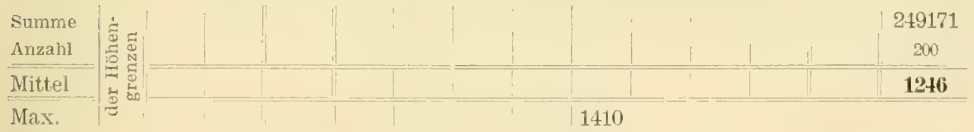

$\mathrm{c}_{1}$ ) obere Grenze der Bestandbildung, als Waldgrenze

\begin{tabular}{|c|c|c|c|c|c|c|c|c|c|c|c|c|}
\hline Summe & 1366 & 8927 & 10293 & 4996 & 16755 & 16808 & 11517 & 10165 & 5196 & 3639 & 69076 & 79369 \\
\hline Anzahl & 1 & 7 & 8 & 4 & 13 & 13 & $\begin{array}{l}9 \\
\end{array}$ & 8 & 4 & 3 & 54 & 62 \\
\hline $\begin{array}{l}\text { Mittul } \\
\text { Max. }\end{array}$ & $136 \mathrm{fi}$ & $127 i$ & 1287 & 1249 & $1289^{\circ}$ & 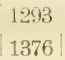 & 1280 & 1271 & 1299 & 1213 & 12.9 & 1280 \\
\hline
\end{tabular}

$\mathrm{c}_{2}$ ) obere Grenze der Bestandbildung, darüber Fichte

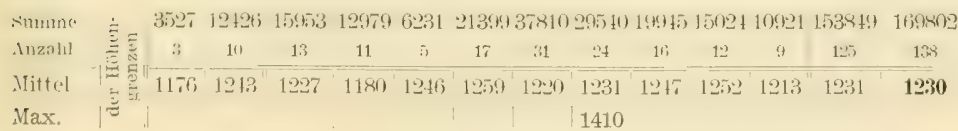




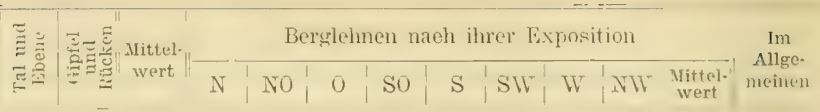

d) sporadisches Vorkommen im Fichtenbestand, obere Grenze

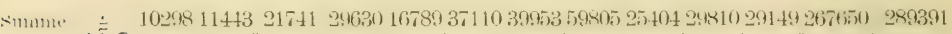

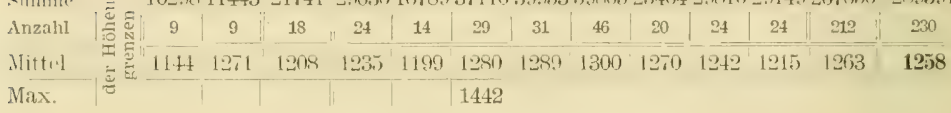

e) Strauchform, obere Grenze

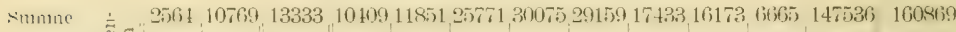
Anzalul

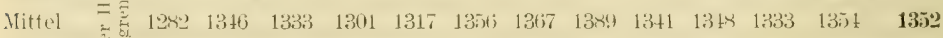
Max. $|\stackrel{\Xi}{\Xi}| 1481$

\section{Quercus sessiliflora Salisb.}

a) rein, oder vorherrschend, obere Grenze

\begin{tabular}{|c|c|c|c|c|c|c|c|c|c|c|c|c|c|c|}
\hline Stumme & & 1152 & 4463 & 5615 & 595 & 732 & 2125 & 4453 & 10278 & 11783 & 5910 & 785 & 36661 & 42276 \\
\hline Anzahl & : & 2 & 7 & 9 & 1 & 1 & 3 & 7 & 14 & 16 & 8 & 1 & 51 & 60 \\
\hline $\begin{array}{l}\text { Mitti- } \\
\text { Max. }\end{array}$ & 5 & 5.6 & 638 & $6 \geq 4$ & 505 & 732 & 708 & 636 & 734 & 736 & 739 & $\left(x_{i}\right)$ & 719 & 70.3 \\
\hline
\end{tabular}

b) vereinzelt (Baumform), obere Grenze

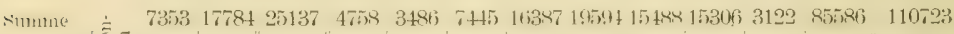

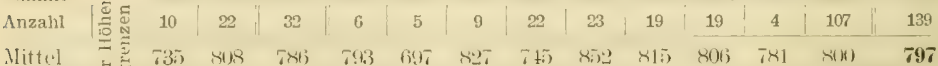

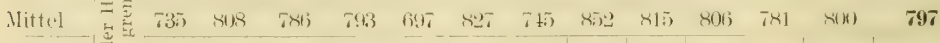
Max.

c) verkriippelt, obere Grenze*

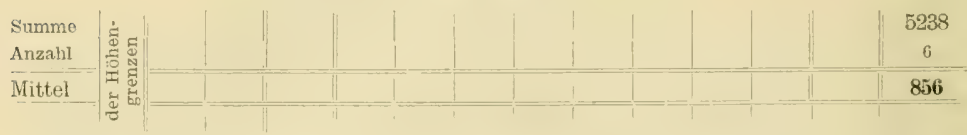

Quereus pedunculata Ehrh. (Qu. Robur L.)

a) rein, oder vorherrschend, obere Grenze

\begin{tabular}{|c|c|c|c|c|c|c|c|c|}
\hline Summo & 388 & 592 & 690 & 2140 & 2453 & 644 & 690 & 7597 \\
\hline AnzahI & 1 & 1 & 1 & 3 & 4 & 1 & 1 & 12 \\
\hline Mittel & 388 & 502 & 690 & 713 & 613 & $6 H$ & 690 & 633 \\
\hline Max. & & & & 720 & & & & \\
\hline
\end{tabular}

* Das maximum ist kleiner als bei der Baumform. 
b) vereinzelt, obere Grenze

\begin{tabular}{|c|c|c|c|c|c|c|c|c|c|c|c|c|c|}
\hline Summe & & 1515 & 3560 & 5075 & 744 & 340 & 1961 & 5460 & 2582 & 2030 & 560 & 13677 & 18752 \\
\hline Anzalil & Е & 2 & 5 & 7 & 1. & 1 & 4 & 7 & 4 & 4 & 1 & -2 & $2: 4$ \\
\hline Mittel & $1=\frac{5}{E}$ & ins & 713 & 725 & 74 & $3+0$ & $4(4)$ & 780 & istij & $50 \mathrm{x}$ & Eiti) & $\left(i^{2} \cdot 2\right)$ & $6+7$ \\
\hline Max. & $\pi$ & & 951 & & & & & & & & & & \\
\hline
\end{tabular}

\section{Quercus lanuginosa Lam.}

a) bestandbildend, obere Grenze

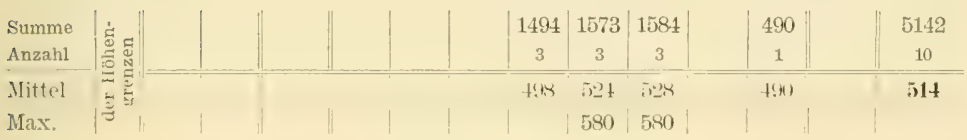

b) vereinzelt, obere Grenze

\begin{tabular}{l|l|l|l|l|l|l|l||c|}
$\begin{array}{l}\text { Summe } \\
\text { Anzahl }\end{array}$ \\
\hline Mittel \\
\hline Max.
\end{tabular}

\section{Quercus Cerris $\mathbf{L}$.}

a) bestandbildend, obere Grenze

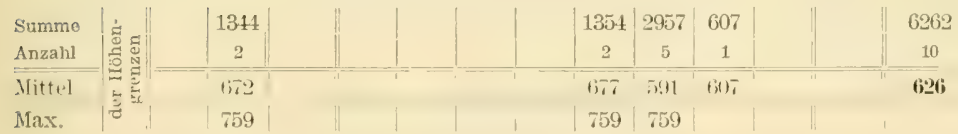

b) vereinzelt, obere Grenze

\begin{tabular}{|c|c|c|c|c|c|c|c|c|c|c|c|c|}
\hline Simmone & 4080 & 4080 & $7(60)$ & 1489 & 15110 & B?titi & $5 x)(x$ & inkr? & 2634 & $T(j)$ & 21121 & $2=214$ \\
\hline Anzahil & 5 & 5 & 1 & 2 & 2 & 6) & 7 & 8 & 3 & 1 & 30 & (i) \\
\hline Mittel & 816 & 816 & 760 & 745 & 755 & 661 & 715 & 625 & 880 & 760 & 704 & $7 \geq 0$ \\
\hline Max. & & & & & & & & & 981 & & & \\
\hline
\end{tabular}




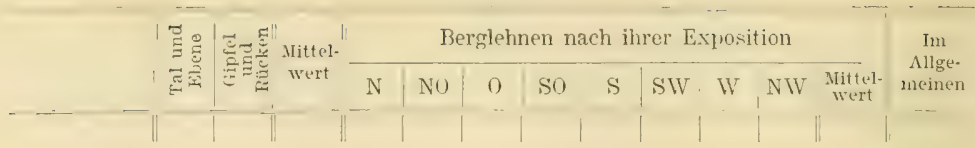

Carpinus Betulus $\mathbf{I}$.

a) bestandbildend, obere Grenze

\begin{tabular}{|c|c|c|c|c|c|c|c|c|c|c|c|c|c|c|}
\hline Summe & & 537 & 1269 & 1806 & 620 & 1553 & 495 & 1985 & 836 & 2032 & 1802 & 1600 & 10933 & 12729 \\
\hline Anzahl & & 1 & 2 & 3 & 1 & 3 & 1 & 3 & 1 & 3 & 3 & 3 & 17 & 20 \\
\hline Mittel & 4 & 537 & 635 & 602 & 620 & 777 & 49.5 & 662 & 836 & 677 & 601 & (4i1) & 643 & 6.36 \\
\hline Max. & 10 & & & & & & & & & & & 920 & & \\
\hline
\end{tabular}

b) vereinzelt, obere Grenze

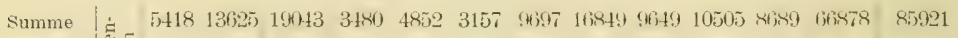

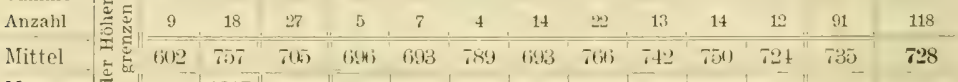
Max.

\section{Corylus Arellana I.}

obere Grenze

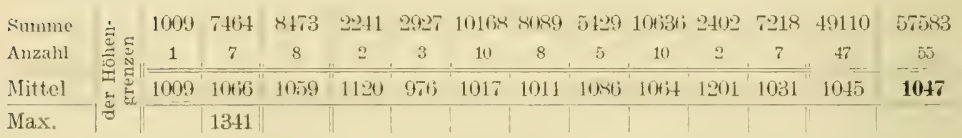

\section{Betula verrucosa Flurh.}

obere Grenze

\begin{tabular}{|c|c|c|c|c|c|c|c|c|c|c|c|c|}
\hline Summe & 2228 & 2228 & 5908 & 7172 & 5607 & 8982 & 6881 & 8165 & 6958 & 4088 & 53861 & 56089 \\
\hline nzahl & 2 & 2 & 5 & 6 & 5 & 8 & fi & 7 & (j) & + & 47 & 49 \\
\hline littel & $=1114$ & 1114 & $11 \times 2$ & 1195 & $11+1$ & 1123 & 1147 & 1166 & $1160)$ & 1022 & $11+1 j$ & 1145 \\
\hline Max. & & & & & & & & & 1478 & & & \\
\hline
\end{tabular}

\section{Betula pubescens Ehrh.}

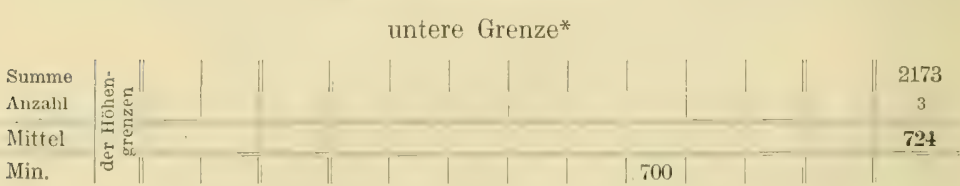

* Bezieht sich nur auf die Hohe Tátra. 


\begin{tabular}{|c|c|c|c|c|c|c|c|c|c|c|c|c|}
\hline \multirow{2}{*}{ 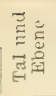 } & \multirow{2}{*}{ 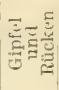 } & \multirow{2}{*}{$\begin{array}{l}\text { Nittel } \\
\text { wert }\end{array}$} & \multicolumn{9}{|c|}{ Pergleluen nach ihrer Expositiun } & \multirow{2}{*}{$\begin{array}{c}\text { IuI } \\
\text { Allge- } \\
\text { meinen }\end{array}$} \\
\hline & & & $\mathrm{N}$ & NO & 0 & So & S & SW & W & NW & $\begin{array}{c}\text { Mittel- } \\
\text { wert }\end{array}$ & \\
\hline
\end{tabular}

obere Grenze*

\begin{tabular}{|c|c|c|c|c|c|c|c|c|c|c|c|c|c|}
\hline Summe & & 2774 & 2774 & 1423 & 1486 & 1521 & 1579 & 2951 & 4633 & 3010 & 4539 & 21145 & 23919 \\
\hline Anzah & & 2 & 2 & 1 & 1 & 1 & 1 & 2 & 3 & 2 & 3 & 14 & 16 \\
\hline Mitte & & 132 & $13 \%$ & 1423 & $148 \mathrm{rij}^{\circ}$ & 159.4 & 1ริด & 1.tits & 15H & $150 \%$ & 1513 & 1510 & 149.5 \\
\hline tax & & & & & & & & & & 1610 & & & \\
\hline
\end{tabular}

\section{Alnus glutinosa Gärtn.}

\begin{tabular}{|c|c|c|c|c|c|c|c|c|c|c|c|c|c|}
\hline $\begin{array}{l}\text { Summe } \\
\text { Anzahil }\end{array}$ & $\dot{\Phi}$ & $\mid \begin{array}{c}34940 \\
\text { th }\end{array}$ & $\begin{array}{c}778 \\
1\end{array}$ & $\mid \begin{array}{c}35718 \\
4 \pi\end{array}$ & $\left|\begin{array}{c}1630 \\
\Xi\end{array}\right|$ & $\left|\begin{array}{c}1154 \\
1\end{array}\right|$ & $\left|\begin{array}{c}2190 \\
3\end{array}\right|$ & $\left|\begin{array}{c}4345 \\
5\end{array}\right|$ & $\left|\begin{array}{c}1200 \\
2\end{array}\right|$ & $\left|\begin{array}{c}1785 \\
2\end{array}\right|$ & $\left|\begin{array}{c}1010 \\
1\end{array}\right|$ & $\begin{array}{c}13314 \\
16\end{array}$ & 49032 \\
\hline Mittel & 55 & 760 & $7 \pi 8$ & itio & .415 & $11 i 1$ & 730 & (kis) & 600 & א.13 & 1010 & ‥3 & 778 \\
\hline Nax. & & $|1290|$ & & & & & & & & & & & \\
\hline
\end{tabular}

\section{Alums incama Willl.}

obere Grenze

\begin{tabular}{l|l|c|c|c|c||c|} 
Summe \\
Anzalil
\end{tabular} Mittul

\section{Salix Caprea $\mathrm{L}_{\text {. }}$.}

obere Grenze

\begin{tabular}{|c|c|c|c|c|c|c|c|c|c|c|c|c|c|c|}
\hline summe & & 5013 & 4011 & 9057 & 8,93 & 1395 & $(x+1)$ & 9381 & $1: 3.3$ & $317 !$ & $130 x$ & $x+1$ & .33k & Sustii) \\
\hline Anzahl & $\frac{0}{0}$ & 4 & 3 & 7 & 7 & 11 & 7 & 7 & 10 & 7 & 1 & 7 & 57 & int \\
\hline Mittel & $\exists=$ & $12(i) 1$ & 1338 & 1394 & 12:4i & 12,1 & $120 i$ & $13 \pm 0$ & 1:24. & 1311 & $133 \mathrm{~K}$ & 13210 & $123 i$ & 1287 \\
\hline Max. & & & & & & & & & & 1451 & & & & \\
\hline
\end{tabular}

\section{Salix silesiaca Willd.}

untere Grenze

\begin{tabular}{|c|c|c|c|c|c|c|c|c|c|}
\hline Summe & & 7618 & 460 & 1052 & 900 & 1136 & 970 & 1707 & 13813 \\
\hline Anzahl & 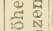 & 8 & 1 & 1 & 1 & 1 & 1 & 2 & 15 \\
\hline Nittel & =5 & 52 & $460)$ & 1052 & $(K) 0$ & 1136 & $9 \pi 0$ & 8.14 & 923 \\
\hline Min. & |z & & 460 & & & & & & \\
\hline
\end{tabular}

* Bezieht sich nur auf die Hohe Tátra. 


\begin{tabular}{|c|c|c|c|c|c|c|c|c|c|c|c|c|}
\hline \multirow{2}{*}{ 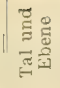 } & \multirow{2}{*}{ 总它 } & \multirow{2}{*}{$\begin{array}{c}\text { Mittel- } \\
\text { wert }\end{array}$} & \multicolumn{9}{|c|}{ Berglehnen nach ihrer Exposition } & \multirow{2}{*}{$\begin{array}{c}\text { Im } \\
\text { Allge- } \\
\text { nleinen }\end{array}$} \\
\hline & & & $\mathrm{N}$ & Ni) & ก & SO & S & SIV & WV & $\mathrm{NHV}$ & $\begin{array}{l}\text { Mittel- } \\
\text { wert }\end{array}$ & \\
\hline
\end{tabular}

obere Grenze

\begin{tabular}{|c|c|c|c|c|c|c|c|c|c|c|c|c|c|c|}
\hline Summe & & 1560 & 4787 & $63+7$ & 4945 & 3158 & $539 \%$ & 1725 & 5316 & $66+19$ & 9575 & 8170 & $4.193 \pi$ & 51282 \\
\hline Anzalıl & 总水 & 1 & 3 & + & 3 & 2 & 3 & 1 & 3 & 4 & 6 & 5 & 87 & 31 \\
\hline Mittel & \pm & 1560 & $15(4)$ & 1 ix & litits & 1579 & 1793 & 17.25 & 1772 & $16(6)$ & $159 \%$ & 1631 & $16 t i t$ & 1654 \\
\hline Max. & 0 & & & & & & & & 1978 & & & & & \\
\hline
\end{tabular}

\section{Populus tremula $\mathbf{I}_{\text {. }}$}

a) Baumform, obere Grenze

\begin{tabular}{|c|c|c|c|c|c|c|c|c|c|c|c|c|c|c|}
\hline Summe & & $510 t$ & 1190 & 6394 & Fisti2 & 3047 & (4) 40 & 7060 & Siti:- & 1013 & +136 & 4301 & $+4 \pi \times 3$ & $5018 \div$ \\
\hline Anzalil & & 5 & 1 & 6 & 5 & 3 & 9 & 7 & 7 & 1 & 4 & 4 & 40 & 4it \\
\hline Mittel & & 10:21 & $11(n)$ & $10+9$ & 1172 & 1016 & 1066 & 10.4 & 1239 & 1013 & $110 . t$ & $107(5)$ & 1115 & 1106 \\
\hline Max. & & & & & & & & & 1425 & & & & & \\
\hline
\end{tabular}

b) Strauchform, obere Grenze*

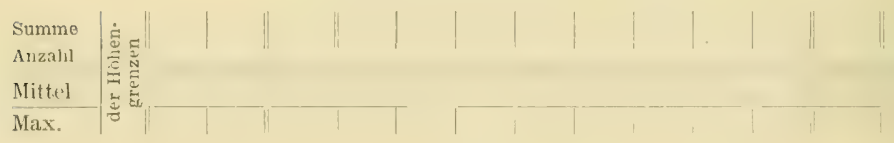

\section{UImus montana With. (U. seabra Mill.)}

a) untere Grenze

\begin{tabular}{l|l|c|c|c|c|c|c|c}
$\begin{array}{l}\text { Summe } \\
\text { Anzahl }\end{array}$ \\
\hline $\begin{array}{l}\text { Mittel } \\
\text { Min. }\end{array}$
\end{tabular}

b) obere Grenze (Baumform)

\begin{tabular}{|c|c|c|c|c|c|c|c|c|c|c|c|c|c|c|}
\hline Summe & & 150391 & 4501 & 90192 & $(i \overline{3} 11)$ & 2075 & 13125 & 17.790 & 15739 & $(45)$ & 1206 & $=30 x$ & $76+31$ & 36883 \\
\hline Anzahl & & 16 & 4 & 20 & 6 & 2 & 13 & 17 & 15 & 9 & 9 & 2 & 73 & 93 \\
\hline Mittel & & $9 \times 1$ & $112 \pi$ & 1010 & 1(Ki) & $113 K$ & 1010 & $10+t i$ & 1649 & $10 \pi$ & 1030 & 1130 & 10 th & 1040 \\
\hline Max. & تै & & & & & & & (i) & & & & 1280 & & \\
\hline
\end{tabular}

* Maximum bei der Baumform. 


\begin{tabular}{|c|c|c|c|c|c|c|c|c|c|c|c|}
\hline \multirow{2}{*}{ 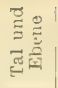 } & \multirow{2}{*}{\multicolumn{2}{|c|}{ 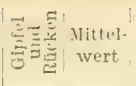 }} & \multicolumn{8}{|c|}{ Rerglehnen nach ihrer Exposition } & \multirow{2}{*}{$\begin{array}{l}\text { Inn } \\
\text { Nittel-: meinen } \\
\text { wert }\end{array}$} \\
\hline & & & $N$ & NO & 0 & SO & S & SIV & W & NW & \\
\hline
\end{tabular}

\section{Tilia parvifolia Elırh. (T. cordata Mill.)}

\begin{tabular}{c} 
obere Grenze \\
$\begin{array}{l}\text { Summe } \\
\text { Anzahl }\end{array}$ \\
\hline Mittel \\
Max.
\end{tabular}

\section{Acer platanoides $\mathbf{L}$.}

a) Baumform, obere Grenze

\begin{tabular}{|c|c|c|c|c|c|c|c|c|c|c|c|c|c|c|}
\hline Sumune & & 4683 & 9143 & 11132 & 2010 & 1933 & 9161 & T:35 , & 11539 & $6+6 i t$ & 10945 & 3313 & $5271 \overline{0}$ & 66857 \\
\hline Anzahl & $\stackrel{0}{0}$ & 5 & 9 & 14 & 2 & 2 & 9 & 7 & 11 & 6 & 10 & 3 & 50 & 64 \\
\hline Mittel & 65 & $93 \mathrm{X}$ & $10+y$ & 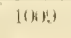 & IIxIs & $(x-3$ & 1018 & 10134 & $10+9$ & 107.7 & 1100 & 1114 & 1()$\left._{i}\right) t$ & $10+4$ \\
\hline Max. & & & & & & & & & & & & $1: 2 \times 6$ & & \\
\hline
\end{tabular}

b) Strauchform, obere Grenze

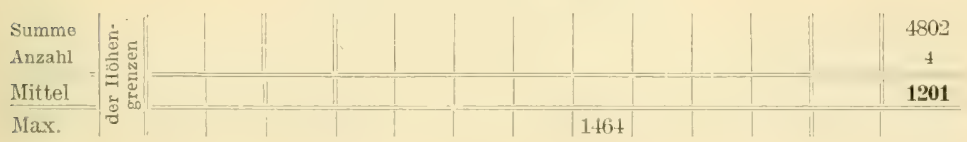

\section{Acer Psendoplatanus L.}

a) Baumform, obere Grenze

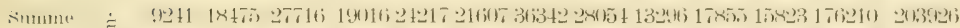

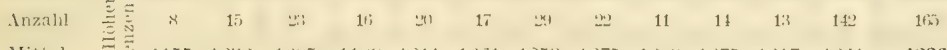
Mittel Max.

b) verkrüppelt, obere Grenze

\begin{tabular}{|c|c|c|c|c|c|c|c|c|c|c|c|c|c|c|}
\hline Summo & & 1333 & 5470 & 6803 & 1266 & $122+8$ & 10821 & 13153 & 10336 & 4826 & 5273 & 6715. & 64538 & 71311 \\
\hline Anzahl & $\begin{array}{l}0 \\
0 \\
0\end{array}$ & 1 & 4 & 5 & 1 & 9 & 8 & 10 & 8 & 4 & 4 & 5 & 49 & 54 \\
\hline $\begin{array}{l}\text { Mittul } \\
\text { Mix. }\end{array}$ & 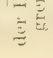 & 1333 & Listix & 13ril & $12 t i t j$ & $\begin{array}{l}1 \text { litil } \\
1 ;(i)\end{array}$ & 13.3 & 131.) & $12 x^{\prime}$ & 1207 & $131 x$ & 1313 & $131 \%$ & 1321 \\
\hline
\end{tabular}




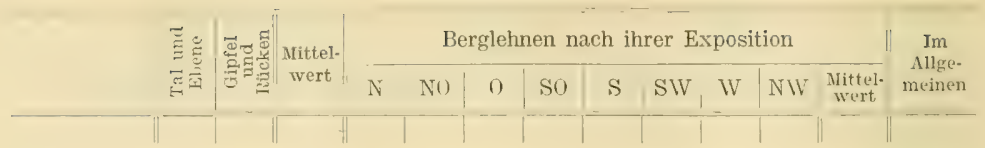

\section{Acer campestre I.}

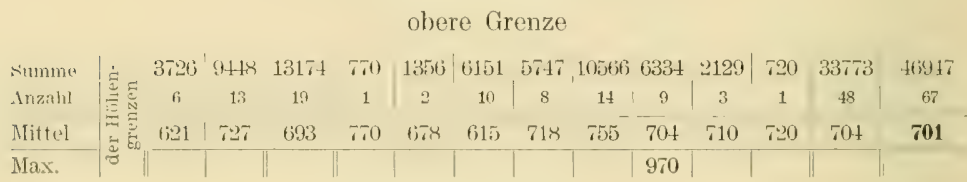

\section{Fraxinus excelsior $I_{4}$.}

\begin{tabular}{|c|c|c|c|c|c|c|c|c|c|c|c|c|c|c|}
\hline Summe & & 3758 & 12694 & 16452 & 890 & 4968 & $7 \pm 74$ & 13326 & 14808 & | 8568 & 4179 & 1195 & 55108 & 71860 \\
\hline Anzahl & 造 & 4 & 12 & 16 & 1 & 5 & 8 & 13 & 14 & 9 & 4 & 1 & in & 71 \\
\hline Mitt.eI & Th & 940 & 1058 & 1028 & 890 & 994 & 934 & 1025 & 1058 & 952 & 1045 & 1195 & 1007 & 1012 \\
\hline Max. & | & & 1227 & & & & & & $\cdot 1$ & & & & & \\
\hline
\end{tabular}

\section{Sorbus anemparia $\mathbf{L}_{\text {. }}$}

a) Baumform, obere Grenze

\begin{tabular}{|c|c|c|c|c|c|c|c|c|c|c|c|c|c|c|}
\hline Sumne & & ․․ำ & $39 k 4$ & 12514 & 22387 & 21246 & 34007 & $12: 95$ & 6896 & 12602 & 9155 & 13002 & $12+590$ & 137104 \\
\hline Anzahlıl & & 7 & 3 & 10 & 17 & 19 & 18 & 9 & 5 & 9 & 7 & 10 & 94 & 104 \\
\hline Mittel & & 1218 & 1329 & 1951 & 1317 & 1276 & 1331 & 1366 & 1379 & 1400 & 1308 & 1300 & 1325 & 1318 \\
\hline Max. & & & & & & & & & 1581 & & & & & \\
\hline
\end{tabular}

b) allgemeine obere Grenze

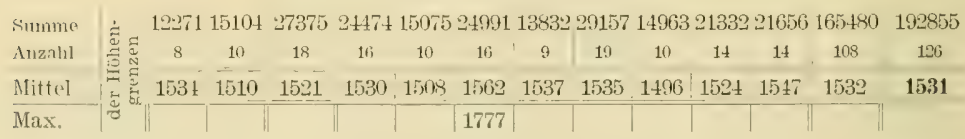

\section{Sorbus Aria Cr.}

a) untere Grenze

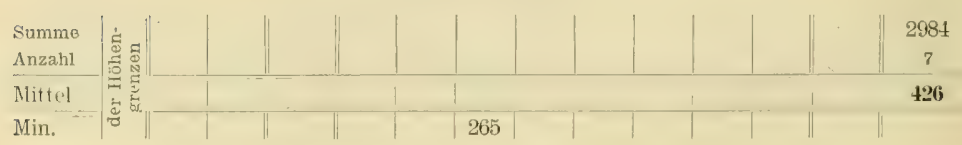




\begin{tabular}{|c|c|c|c|c|c|c|c|c|c|c|c|c|}
\hline \multirow{2}{*}{ 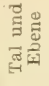 } & \multirow{2}{*}{ పี心 } & \multirow{2}{*}{$\begin{array}{c}\text { Mittel- } \\
\text { wert }\end{array}$} & \multicolumn{9}{|c|}{ Berglehnen nach ihrer Exposition } & \multirow{2}{*}{$\begin{array}{c}\text { Im } \\
\text { Allge. } \\
\text { meinen }\end{array}$} \\
\hline & & & $\mathrm{N}$ & NO & 0 & SO & S & SW & W & NWV & $\begin{array}{l}\text { Mittel. } \\
\text { wert }\end{array}$ & \\
\hline
\end{tabular}

b) obere Grenze

\begin{tabular}{|c|c|c|c|c|c|c|c|c|c|c|c|}
\hline Summe & & 4083 & 3864 & 1389 & 2649 & 4009 & 4935 & 2449 & 2680 & 5269 & 31327 \\
\hline Anzahl & : & 3 & 3 & 1 & 2 & 3 & 4 & 2 & 2 & 4 & 24 \\
\hline Mittel & $\div$ & $137 i 1$ & 1289 & 1389 & 1325 & 1336 & 1234 & 1225 & 1340 & 1317 & 1305 \\
\hline Max. & | & 1395 & & & & & & & & & \\
\hline
\end{tabular}

\section{Crataegus monogyna Jace. und oxyacantha $\mathbf{L}$.}

\begin{tabular}{lccccccccccccc}
\multicolumn{10}{c}{ obere Grenze } \\
$\begin{array}{l}\text { Summe } \\
\text { Anzahi }\end{array}$ \\
$\begin{array}{l}\text { Mittel } \\
\text { Max. }\end{array}$
\end{tabular}

\section{Sambueus racemosa L.}

a) untere Grenze

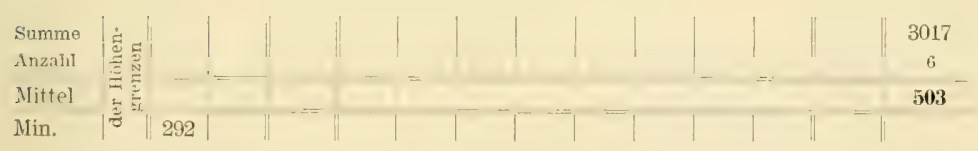

b) obere Grenze

\begin{tabular}{|c|c|c|c|c|c|c|c|c|c|c|}
\hline Sunme & & 1163 & $13: 1$ & 1250 & $1: 30$ & $7 \times 32$ & 5076 & 2401 & 1:339 & 23015 \\
\hline Anzahl & $\frac{\mathscr{3}}{5}$ & 1 & 1 & 1 & 1 & 6 & 4 & 2 & 1 & 17 \\
\hline Nittel & $=\vec{g}$ & $1163^{\prime}$ & 1321 & 1200 & 1230 & $130 \pi$ & 1269 & 145 & 1239 & 1295 \\
\hline Max. & & & & & & & & 1506 & & \\
\hline
\end{tabular}




\section{Nordostkarpathen.}

Hoverla 2058 m., Pop Iván 1910 m., Szvidovec 1883 m., Sztoj 1679 m., Polonina Runa $1482 \mathrm{~m}$., Guttin $1447 \mathrm{~m}$.

\begin{tabular}{|c|c|c|c|c|c|c|c|c|c|c|}
\hline \multirow{2}{*}{ 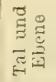 } & \multirow{2}{*}{ 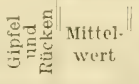 } & \multicolumn{8}{|c|}{ Berglehnen nach ihrer Exposition } & \multirow{2}{*}{$\begin{array}{l}\text { Im } \\
\text { Allge- } \\
\text { meinen }\end{array}$} \\
\hline & & NO & & & & & & & & \\
\hline
\end{tabular}

Picea excelsa Lk.

a) vereinzelt, untere Grenze

\begin{tabular}{|c|c|c|c|c|c|c|c|c|c|c|c|c|}
\hline Summe & $25+3$ & $25+3$ & איאו4 & 4155 & 6745 & 3191 & 5355 & 5989 & 5151 & $27+2$ & 37536 & 40079 \\
\hline Anzahl & 4 & 4 & 7 & 7 & 11 & 5 & 8 & 9 & 8 & 5 & 60 & 64 \\
\hline Mittel & 636 & $63 b^{\circ}$ & 601 & 591 & 613 & 638 & 669 & 665 & 644 & $5+8$ & 626 & 626 \\
\hline Min. & 370 & & & & & & & & & & & \\
\hline
\end{tabular}

b) rein, oder vorherrschend, untere Grenze

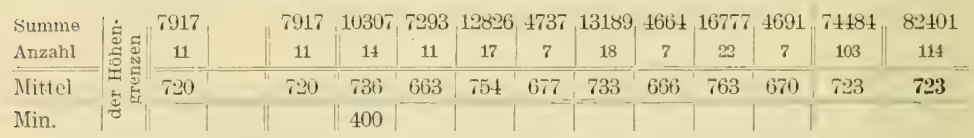

c) geschlossener Bestand, obere Grenze

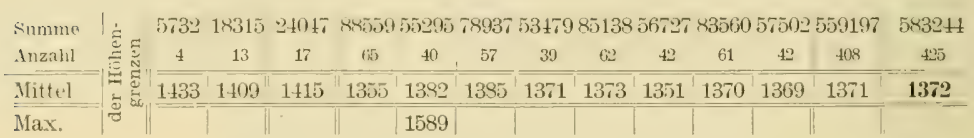

d) Raumbestand, obere Grenze (Waldgrenze)

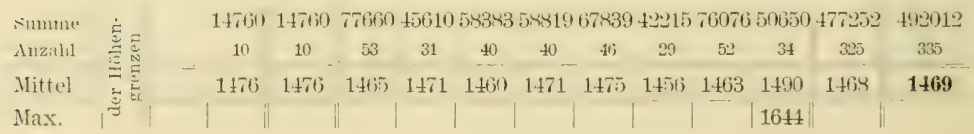




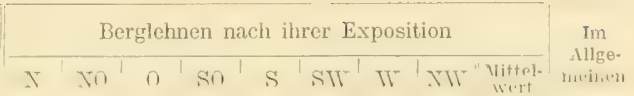

e) obere Baumgrenze

(Bäume mit $8 \mathrm{~m}$. Gipfelhölı.)

\begin{tabular}{|c|c|c|c|c|c|c|c|c|c|c|c|c|c|c|}
\hline sumume & & 147.3 & Silts & $1111: 1$ & (4):201 & {$[,(1,3,3]$} & 16:xla & ifilli: & $3,4,4=31$ & 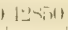 & (1)?,3.3 & $1,1.3 .3$ & $\because 1 \%$ & $. x=1<?$ \\
\hline Anzahl & $\stackrel{0}{0}$ & 1 & 6 & 7 & 64 & 34 & 41 & 41 & 60 & | 29 & 65 & 50 & 397 & 394 \\
\hline Mittel & $=5$ & 14.2 & $1+41$ & 1146 & $11 \times 8$ & $1+\times-7$ & 14. & $11: 31$ & $14: 12$ & $14 \pi x$ & $1+7 t$ & $14: 1$ & 114, & $1+8 \bar{i}$ \\
\hline $\operatorname{Max}$. & चै & & & & & & & & & & 1675 & & & \\
\hline
\end{tabular}

f) geschlossener Bestand mit rerkrüppelten Fichten, obere Grenze

\begin{tabular}{|c|c|c|c|c|c|c|c|c|c|c|c|c|c|}
\hline Summe & & 4668 & 4668 & 6381 & 7771 & 1567 & 6257 & 10916 & $4590^{\circ}$ & 21954 & 8031 & 67497 & 72165 \\
\hline Anzahl & $\frac{\mathrm{d}}{3} \underbrace{\underline{5}}_{\mathrm{N}}$ & 3 & 3 & 4 & 5 & 1 & 1 & 7 & 3 & 14 & 5 & 43 & 4h, \\
\hline Mittel & $\exists$ & $150 \pi$ & 1initi & 1545 & 1.251 & 1 inti & 1.5151 & linit & $(2,3)^{\prime}$ & 1568 & 1600 & $\operatorname{lin} 0$ & 1568 \\
\hline Max. & T & & & & & & & & & 1673 & & & \\
\hline
\end{tabular}

g) rerkrüppelt, obere Grenze

\begin{tabular}{|c|c|c|c|c|c|c|c|c|c|c|c|c|c|}
\hline Summe & & Eyyy & 6939 & 10618 & $\varnothing>63$ & 10008 & $1056=$ & 2303 & $1060^{2}-1$ & 12001 & 3336 & $8+3,0$ & 11,12 \\
\hline Anzahl & 동 & 4 & 4 & 9 & 5 & 9 & 6 & 3 & 6 & 7 & 3 & 48 & 52 \\
\hline Mittel & 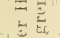 & 1.ix & 17.) & 1739 & $17-3$ & $17 \because 4$ & 1 (iti) & litiv & 17,11 & 1793 & 17.9 & 1.1395 & 1767 \\
\hline Max. & $\left|\begin{array}{ll}* & 1\end{array}\right|$ & & & 1. & 1930 & & & & & & & & \\
\hline
\end{tabular}

\section{Abies pectimata DC. (A. alba Mill.)}

a) untere Grenze

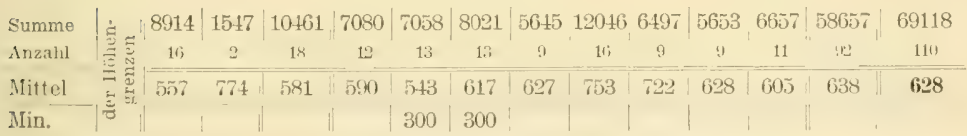

b) rein, oder vorherrschend, obere Grenze

\begin{tabular}{|c|c|c|c|c|c|c|c|c|c|c|c|c|c|}
\hline Summe & & 919 & 949 & $55+5$ & 7088 & 3992 & 1816 & 980 & 3527 & 2903 & 4674 & 30525 & $3147 \pm$ \\
\hline Anzahl & $\cong \frac{\overrightarrow{5}}{5}$ & 1 & 1 & 6 & 8 & 4 & 2 & 1 & 4 & 3 & 5 & 33 & $3+$ \\
\hline MitteI & $=5$ & 949 & 949 & 924 & 886 & 998 & 908 & 980 & 882 & 968 & $93 \overline{i)}$ & 935 & 926 \\
\hline Max. & & & & & & 1326 & & & & & & & \\
\hline
\end{tabular}

c) vereinzelt, obere Grenze

\begin{tabular}{|c|c|c|c|c|c|c|c|c|c|c|c|c|c|c|}
\hline Simu1nu" & & ent & inllli & 17,61 & $(+\infty), n$ & $3+1,0$, & 5.41 .5 & 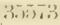 & .11, & $\cdots, 1$, & & $t=1$ & & \\
\hline Anzahl & $\int_{0}^{3}$ & 9 & 5 & 14 & 38 & 30 & 32 & 30 & 42 & 33 & 39 & 36 & 280 & 294 \\
\hline Mittel & $\therefore 5$ & 11811 & 10179 & $1+\pi)$ & 11 tis & $11 ., 1)$ & $1 \geq 21$ & $118 i$ & $1=1 \%$ & $119 ; ;$ & $11 ! H i$ & $1 \mathrm{Lri}^{-7}$ & $114 i$ & 1180 \\
\hline Max. & $=$ & & & & & & & & & 1421 & & & & \\
\hline
\end{tabular}




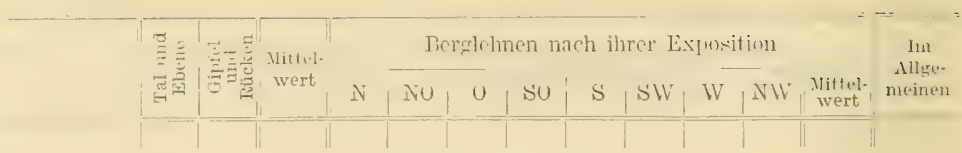

d) verkriippelt, obere Grenze

\begin{tabular}{|c|c|c|c|c|c|c|c|c|c|c|c|c|}
\hline S'munut & 1113 & 4113 & 6.13 & $2 \times 1 \%$ & 6809 & $1085:$ & $533^{\prime} y$ & 105.1 & $110 \%$ & 10936 & $6570 \pi$ & izsil) \\
\hline Anzahl & 3 & 3 & 5 & 2 & 5 & 8 & 6 & 8 & 8 & 8 & 50 & 53 \\
\hline Mittel & $13 \times 1$ & 1341 & 13inis & $1.10 \mathrm{~K}$ & 1310 & $(3+i)$ & $1+23$ & 1354 & 1385 & 1363 & 137.4 & 1375 \\
\hline Max. & & & & & & & 1510 & & & & & \\
\hline
\end{tabular}

\section{Pinus Cembra I.}

a) untere Grenze

\begin{tabular}{l|l|l|l|c|c|c|c} 
Summe \\
Anzahl
\end{tabular}

b) Baumform, obere Grenze

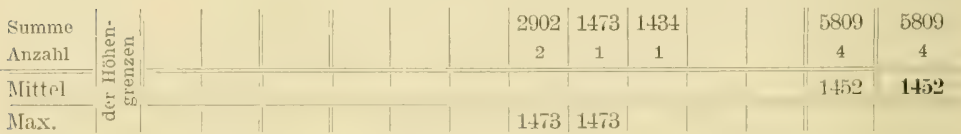

\section{Pinus nontana Mill.}

a) sporadisch, untere Grenze

\begin{tabular}{|c|c|c|c|c|c|c|c|c|c|c|c|c|}
\hline S'uนmo & 1512 & 1512 & $\ddot{21150}$ & 15074 & 1250 & $2 y 3$ & 1389 & 11631 & $17 \geq 10$ & 1333 & 111350 & $11: x(x)$ \\
\hline Anzahl & 1 & 1 & 17 & 11 & 9 & 2 & 10 & 8 & 12 & 9 & 78 & 89 \\
\hline Mittel & 1512 & 1512 & $1+21$ & $1+11 ;$ & Lisitii & 1 thit & lis? & $1+f+1)$ & 1135 & 1142 & 1108 & 1429 \\
\hline Min. & & & & & & & & & 1190 & & & \\
\hline
\end{tabular}

b) massenhaft, untere Grenze

\begin{tabular}{|c|c|c|c|c|c|c|c|c|c|c|c|c|}
\hline siunme & $31: 3$ & $31 \div 1$ & diltiv & 15111 & til ti & $31 \%$ & $1+1 i 17$ & $3 \mid k 3$ & $1: 20$ & $9 \pi 111 ;$ & $37+24$ & $405 \mathrm{k}$ \\
\hline Inzahl & 2 & $\because$ & 4 & 1 & 4 & 2 & 1 & 2 & t & fi & $\because 4$ & $3 i$ \\
\hline Mittel & 1562 & lintis & 1512 & 15111 & 1.33 & $15 \pi \mathrm{K}$ & 1617 & $1,1.2$ & $(5 \pi)$ & likl & 1559 & 1560 \\
\hline Min. & & & 1409 & & & & & & & & & \\
\hline
\end{tabular}




\begin{tabular}{|c|c|c|c|c|c|c|c|c|c|c|c|c|}
\hline \multirow{2}{*}{ 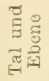 } & \multirow{2}{*}{ 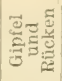 } & \multirow{2}{*}{$\begin{array}{c}\text { Mittel- } \\
\text { wart }\end{array}$} & \multicolumn{9}{|c|}{ Berglehnen nach ihrer Exposition } & \\
\hline & & & $N$ & NO & 0 & $\mathrm{SO}$ & $\mathrm{S}$ & SWV & W & NIV & Mittel- & meinen \\
\hline
\end{tabular}

c) massenhaft, obere Grenze

\begin{tabular}{|c|c|c|c|c|c|c|c|c|c|c|c|c|c|}
\hline Summe & & 3587 & 3587 & 12272 & 7013 & 1791 & 5119 & 5380 & 3520 & 3106 & 1728 & 40229 & 43816 \\
\hline Inzalil & 1 & 2 & 2 & 7 & 4 & 1 & 3 & A & 2 & 12 & 1 & ? & 2 \\
\hline Mittel & $\begin{array}{l}=5 \\
\vdots \\
\Xi 5\end{array}$ & $17: 18$ & 17.48 & 17.3 & $1 \ldots 3$ & $17+1$ & $1 \overline{1} 111 ;$ & $17: 1,3$ & 1i(ii) & 1:19:3 & $172 y$ & $1: 1: 1$ & 17.3:) \\
\hline Vixs. & & & & (Ki) & & & & (1) & & & & & \\
\hline
\end{tabular}

d) sporadisch, obere Grenze

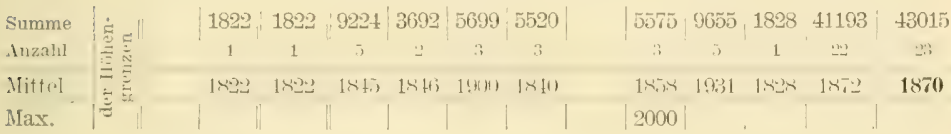

Taxus baceata $I_{\text {. }}$.

a) intere Grenze

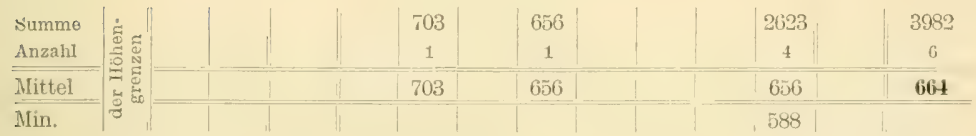

b) obere Grenze

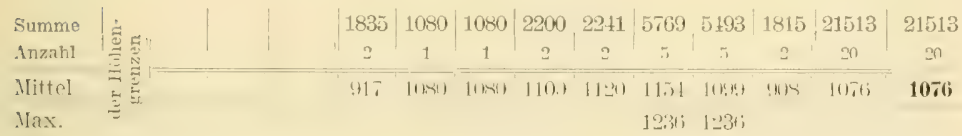

\section{Juniperus communis $\mathbf{L}$.}

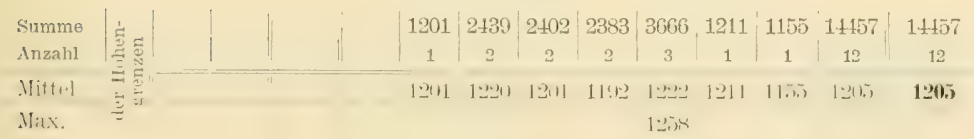




\begin{tabular}{|c|c|c|c|c|c|c|c|c|c|c|c|c|}
\hline \multirow{2}{*}{ 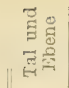 } & \multirow{2}{*}{ 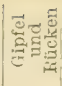 } & \multirow{2}{*}{$\begin{array}{l}\text { Mittel- } \\
\text { wert }\end{array}$} & \multicolumn{9}{|c|}{ Berglehnen nach ihrer Exposition } & \multirow{2}{*}{$\begin{array}{c}\text { Im } \\
\text { Allge- } \\
\text { meituen }\end{array}$} \\
\hline & & & $\mathbb{N}$ & NO & 0 & SO & S & SW & W & NIV & $\begin{array}{l}\text { Mittrl- } \\
\text { wert }\end{array}$ & \\
\hline
\end{tabular}

\section{Juniperus nama Willd.}

a) Intere Grenze

\begin{tabular}{|c|c|c|c|c|c|c|c|c|c|c|c|c|c|}
\hline Nummul & $\dot{\partial}$ & $1(x): 3$ & $11 \times 1: 2$ & tis3i3 & ixis & $1118: 5$ & 19112 & $x 1 ! 14$ & trit & (i) 13 & $3(3 ; 3)$ & ickly & $5 \pi x_{i 1}$ \\
\hline Anzilthl & $\stackrel{\bar{\Xi}}{\Xi}$ & 1 & 1 & 5 & i) & 9 & s & i & 4 & 5 & 3 & $4 i$ & 47 \\
\hline Mittul & 7 & 1012 & $1+1.2$ & $1: 35 \pi$ & $1: 211$ & $1 \div-1 ;$ & $1 \geq 314$ & 11.11 & 1203 & $1: 3(1 !)$ & $13 i f i$ & $1: 3: 36$ & 1231 \\
\hline Min. & & & & & & 703 & & & & & & & \\
\hline
\end{tabular}

b) obere Grenze

\begin{tabular}{|c|c|c|c|c|c|c|c|c|c|c|c|c|}
\hline Summe & $\dot{\pi}$ & 3794 & 3734 & 1831 & 3869 & 3632 & 3795 & 5806 & 3903 & 1818 & 24687 & $28+81$ \\
\hline Anzalil & 负 & 2 & 2 & 1 & 2 & 2 & 2 & 3 & 2 & 1 & 13 & 1.) \\
\hline littul & $=\vec{J}$ & $18 \%$ & $1 \times ! \pi$ & $1<3 i$ & $19 \% 1$ & Lxlti & 1K: & 15iti & 19.11 & 1818 & $18: 4$ & 1899 \\
\hline Iax. & $\mid \vec{z} \quad \|$ & & & & & & & 2000 & & & & \\
\hline
\end{tabular}

\section{Fagus silvatica $\mathbf{l}$.}

a) vereinzelt, untere Grenze

\begin{tabular}{|c|c|c|c|c|c|c|c|c|c|c|c|c|c|}
\hline Summit & & 147 & $1 \bar{k}_{\mathrm{i}}$ & 16331 & और & $x: 10$ & $19+4$ & $15,+3$ & 511. & 6in: & 11811 & 8711 & 88018 \\
\hline Anzahl & 可 & 1 & 1 & 5 & 2 & 3 & 7 & 6 & 3 & 3 & 5 & 34 & 35 \\
\hline Alittel & 45 & $1 \times i$ & $18 \mathrm{in}$ & $321 i$ & 1.X & isil & Pis & 3 titi & $1 \times 1 \mid$ & 203 & ziti & 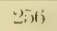 & 254 \\
\hline Min. & 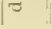 & & & & & 119 & & & & & & & \\
\hline
\end{tabular}

b) bestandbildend, untere Grenze

\begin{tabular}{|c|c|c|c|c|c|c|c|c|c|c|c|c|}
\hline रंmแ1111 & Llivitil & Itivis & $5(10)$ & Hoiv & Elitis & $33 \times 2$ & $1.16 K$ & $24: 37$ & $|x i y|$ & $23+411$ & $2 ! 11) \cdot 93$ & 31729 \\
\hline Anzahl & 5 & 5 & 14 & 12 & 15 & 9 & 4 & 6 & 12 & 10 & 82 & 87 \\
\hline Mittel & $33 i$ & $33 \overline{4}$ & $3: i n$ & 310 & ilti & $3 \pi 6$ & 374 & $\frac{100}{60}$ & tilt & $9 !+4$ & 3ij) & 354 \\
\hline Min. & & & & & & & & & & 124 & & \\
\hline
\end{tabular}

c) Bestandbildung in Allgemeinen, obere Grenze

\begin{tabular}{|c|c|c|c|c|c|c|c|c|c|c|c|c|c|c|}
\hline$\therefore 11 m 1114$ & & t7: & Intingi & 3. & $5(1,3): 4$ & 191376 & 4hist & $T \pi t i 21$ & (Mintis & $(j 125)$ & Somit & tixi & $\pi 16,5$ & (ax) \\
\hline Anzanlal & & 4 & 31 & 24 & div & fil & 76 & (it & 73 & in ! & 13 & 37 & tis & $4 \pi$ \\
\hline Mittel & & $11 \times 2$ & Jב1x & 121" & $123 t$ & 1031 & 1ั7ィ & 1273 & $12+11$ & 1325 & 1212 & $12+11$ & $12+7$ & 1245 \\
\hline Max. & & & & & & & & & 1387 & & & & & \\
\hline
\end{tabular}




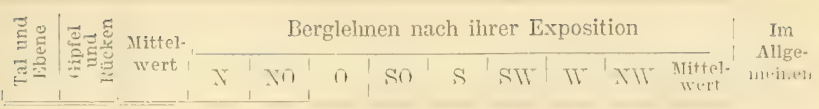

$\mathrm{c}_{1}$ ) obere Grenze der Bestandbildung als Waldgrenze

simme | \begin{tabular}{l} 
Anzahl \\
\hdashline Mittal
\end{tabular} Max, | | | | | | | | |

$\mathrm{c}_{2}$ ) obere Grenze der Bestandbildung, darüber Fichte

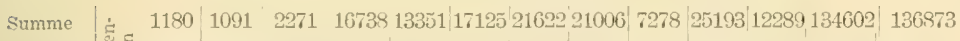

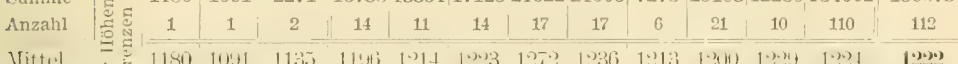
Mittel Max. $\left|\div \frac{1}{|+|}\right| \frac{1375 \mid}{1+1}$

d) sporadisches Vorkommen im Fichtenbestand, obere Grenze

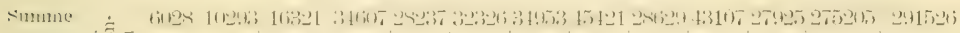

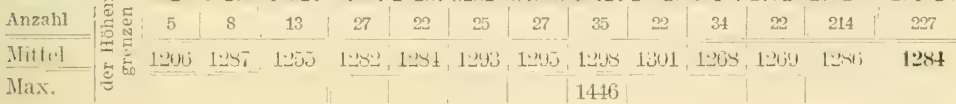

e) Strauchform, obere Grenze

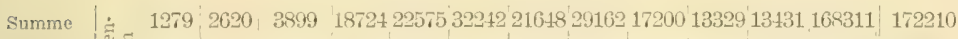

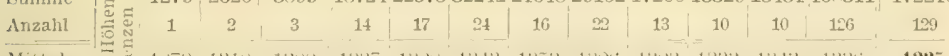

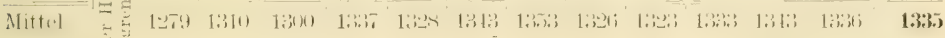

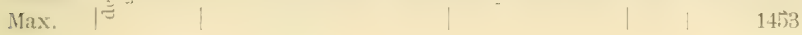

\section{Quereus sessiliflora Salisb.}

a) rein, oder vorherrschend, obere Grenze

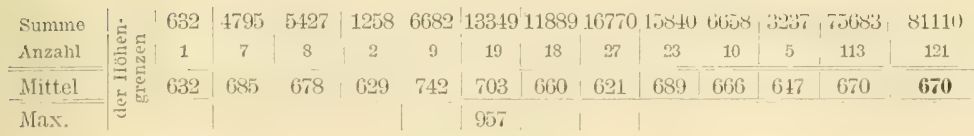

b) vereinzelt (Bumnform), obere Grenze

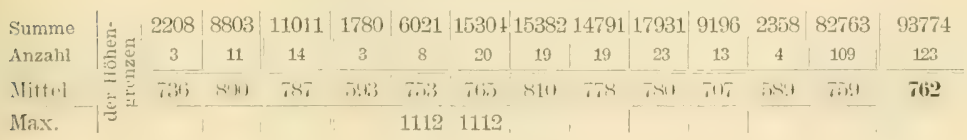




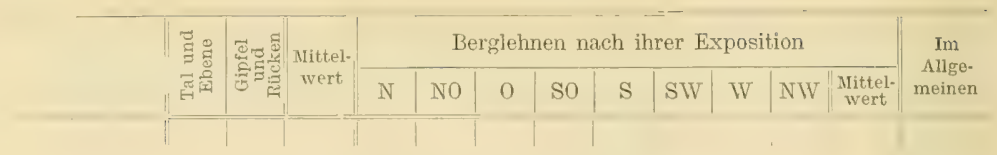

c) verkrüppelt, obere Grenze

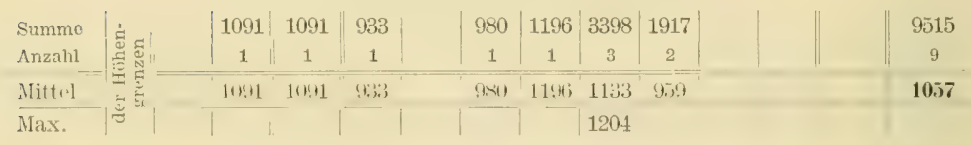

\section{(Quercus perluneulata Ehrh. (Qu. Robur I.)}

a) rein, oder vorherschend, obere Grenze

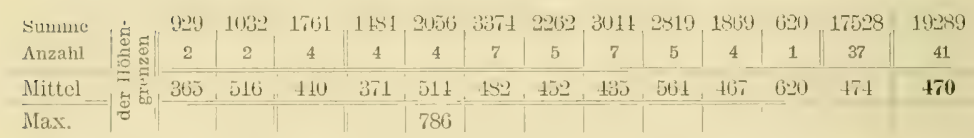

b) vereinzelt, obere Grenze

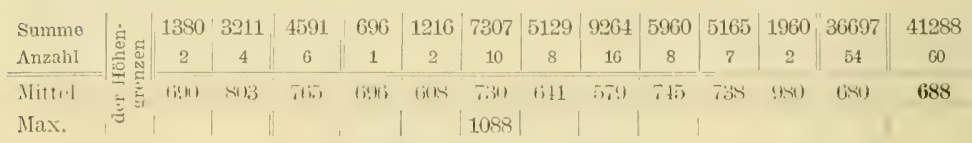

\section{Carpinus Betulus $\mathbf{l}$.}

a) bestandbildend, obere Grenze

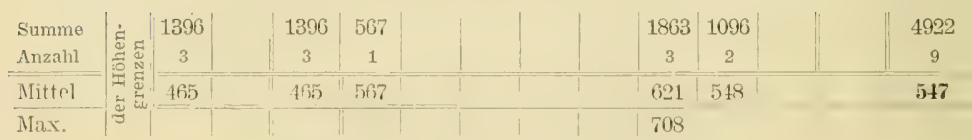

b) rereinzelt (Baumform), obere Grenze

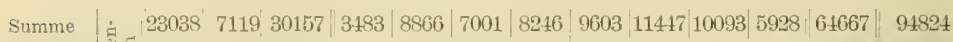
\begin{tabular}{l|ccccccccccccc} 
Anzalil \\
Mittel
\end{tabular} $\operatorname{Max}||^{2}|+| 949 \mid$

c) Strauchform, obere Grenze

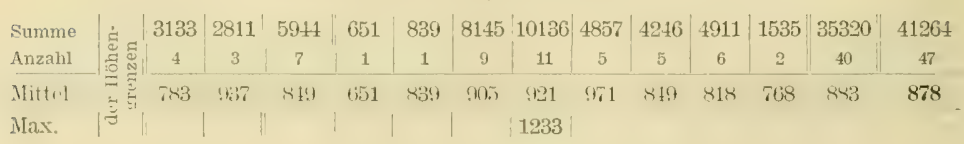




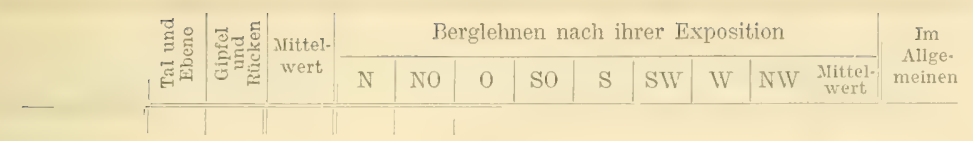

\section{Corylus Irellana h.}

obere Grenze

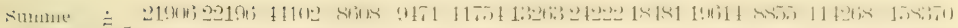

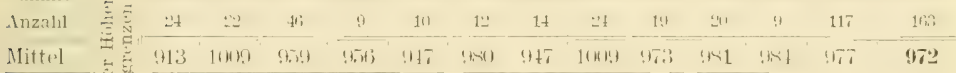

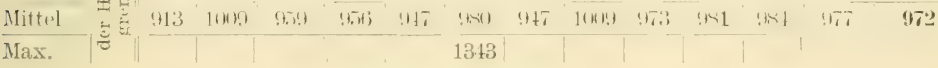

\section{Betula verrucosa Ehrh.}

ohere Grenze

\begin{tabular}{|c|c|c|c|c|c|c|c|c|c|c|c|c|}
\hline Summe & 3810 & 3810 & 6730 & 12916 & 6379 & 10306 & 14060 & 16669 & 9077 & 6462 & 82505 & 86315 \\
\hline Anzalal & 4 & + & fi & 12 & ci & 111 & 11 & 17 & 4 & i & $s t$ & s, \\
\hline Mitfrel & $(1, i 3)$ & 4.23 & 1122 & $117,1 i$ & loHiz; & $1|\mathrm{z}|$ & $1(11 \%)$ & !1 & $11 \times 1: 1$ & (12:3 & $16+1 ! 1$ & 1015 \\
\hline Iax. & & & 1410 & & & & & & & & & \\
\hline
\end{tabular}

\section{Aluus glutinosa Girtı.}

a) Baumform, obere Grenze

\begin{tabular}{|c|c|c|c|c|c|c|c|c|c|c|c|c|}
\hline Summe & \& 14800 & 14802 & 1064 & 4971 & 3372 & 4310 & 2215 & 4985 & 2191 & 550 & 23958 & 38760 \\
\hline Anzahl & 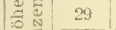 & 39 & 2 & 10 & G & 8 & 4 & 10 & 5 & 1 & 46 & 75 \\
\hline Mitt..l & isll & $\therefore 10$ & (a) & $1: \overline{1}$ & itti2 & in?.1 & init & 1:k & $4 ! K$ & $5, \pi 1$ & TLI & 517 \\
\hline Max. & ま & & & & 870 & & & & & & & \\
\hline
\end{tabular}

b) Strauchform, obere Grenze

\begin{tabular}{|c|c|c|c|c|c|c|c|c|c|c|c|c|}
\hline Sinmme & $13: 3$ & $\therefore-y_{i j}$ & 3nt: & 3353 & $11 \times 1$ & 1472 & 3412 & $1: 111$ & $2+31$ & 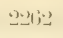 & 151413 & $14+12$ \\
\hline Auzahli & 2 & $\therefore$ & $\therefore$ &. & $\ddot{\prime}$ & 2 & : & $::$ & 4 & $::$ & 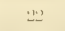 & 27 \\
\hline Mittel & (bi) & it.2 & 710 & $7 \times 4$ & I:11 & initi & (ivi) & ti.i, & (1115) & int & lix? & 687 \\
\hline Max. & & & & 1020 & & & & & & & & \\
\hline
\end{tabular}

\section{Alums incana Willd.}

a) Baumform, obere Grenze

\begin{tabular}{|c|c|c|c|c|c|c|c|c|c|c|c|c|c|c|}
\hline Summe & & 45790 & 5310 & 51100 & 2197 & 10781 & 9683 & $115 \pm 6$ & 7466 & 14515 & 6518 & 2451 & 65193 & 116593 \\
\hline Anzahl & 点 & 53 & 7 & 60 & 3 & 13 & 12 & 14 & 9 & 17 & 8 & 3 & 79 & 139 \\
\hline Nittid & & xilt & 704 & $(x)=$ & $\times 32$ & $\times 31$ & SIF & 831 & 8,31 & sit & $x 1 !$ & ২1২ & 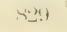 & 839 \\
\hline Iax. & & & & & & & & 1257 & & & & & & \\
\hline
\end{tabular}




\begin{tabular}{|c|c|c|c|c|c|}
\hline \multirow{2}{*}{ 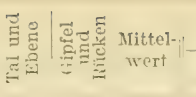 } & \multicolumn{4}{|c|}{ Berglehnen nach ihrer Exposition } & \multirow{2}{*}{$\begin{array}{l}\text { Im } \\
\text { Allge. } \\
\text { meinen }\end{array}$} \\
\hline & Ni) 0 & so & $\mathrm{SW} \mathrm{W}^{\prime} \mathrm{NW}$ & $\begin{array}{l}\text { Mittel- } \\
\text { wert }\end{array}$ & \\
\hline
\end{tabular}

b) Strauchform, obere Grenze

\begin{tabular}{|c|c|c|c|c|c|c|c|c|c|c|c|}
\hline S'แ1นแ11 & 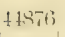 & $4140 ;$ & 1058 & 3138 & 1706 & 2993 & 3731 & 3176 & 1819 & 17421 & 62297 \\
\hline Anzahl & 49 & 49 & 2 & 2 & 2 & 3 & 4 & 3 & 2 & 18 & 67 \\
\hline Mitt.l & 916 & 916 & 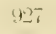 & 1019 & К53 & 994 & 933 & 1059 & 909 & 968 & 930 \\
\hline Max. & 1281 & & & & & & & & & & \\
\hline
\end{tabular}

\section{Aluus virilis 06 .}

a) untere Grenze

\begin{tabular}{|c|c|c|c|c|c|c|c|c|c|c|c|c|c|}
\hline summe & 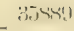 & 4193 & $4(1) \times=3$ & 1.6 .1 & 7316 & $10): 11$ & 6733 & oittio & 8911 & $3661)$ & 91917 & $70 \times 36$ & 11091s \\
\hline Inzahil & 411 & 4 & 44 & $1 i$ & 7 & 111 & i) & 6 & 8 & 3 & 9 & is & 109 \\
\hline Tittral & $8 \div \%$ & 1014 & 911. & $110 t$ & 1114. & $10: 41$ & 1122 & איה & 1025 & $12: 01$ & 1094 & 1010 & 1018 \\
\hline Iin. & 402 & & & & & & & & & & & & \\
\hline
\end{tabular}

b) obere Grenze

\begin{tabular}{|c|c|c|c|c|c|c|c|c|c|c|c|c|}
\hline Summo & 4701 & 4701 & 33766 & 25278 & 27714 & 11290 & 4517 & 4899 & 10919 & 28550 & 146969 & 151670 \\
\hline Anzalul & 3 & 3 & 21 & 15 & 17 & 7 & 3 & 3 & 7 & 18 & 41 & 94 \\
\hline Mitt, l & 50 & litis & lotk & $16 x, 1$ & $10 \ddot{3} ; 0$ & $|t i| t$ & Lithi & 160363 & 15tit & 15,$4 ;$ & 1615 & 1614 \\
\hline Max. & $=1$ & & & & & & & 2000 & & & & \\
\hline
\end{tabular}

\section{Populus tremula 1.}

a) Baumform, obere Grenze

\begin{tabular}{|c|c|c|c|c|c|c|c|c|c|c|c|c|c|}
\hline Summ & & 1875 & 1875 & 8691 & 15107 & 11352 & 6171 & 17575 & 26621 & 12163 & 6130 & 104116 & 105991 \\
\hline Anzahl & & 2 & 2 & 10 & 18 & 13 & 7 & \begin{tabular}{|l}
19 \\
\end{tabular} & 31 & 14 & 7 & 119 & 121. \\
\hline Mitter & & $13 x$ & 934 & sil? & Sint & $\times 73$ & K४: & (2) & $93 ! 4$ & stily & 4.7 & 8.5 & 876 \\
\hline Nax. & & & & & & & & 1204 & & & & & \\
\hline
\end{tabular}

b) Strauchform, obere Grenze

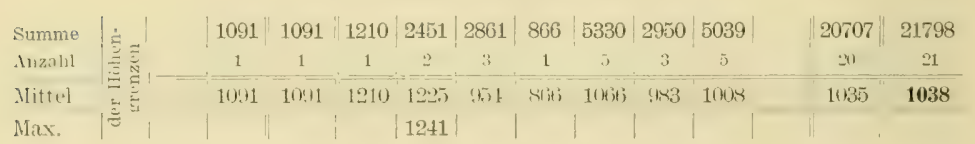




\begin{tabular}{|c|c|c|c|c|c|c|c|c|c|c|c|c|c|}
\hline & $\ddot{g}$ & $\overrightarrow{\mathrm{c}}=$ ह & $\mid$ Vittel- & \multicolumn{9}{|c|}{ Berglehnen nach ihrer Exposition } & \multirow{2}{*}{$\begin{array}{c}\mathrm{Im} \\
\text { Allewe } \\
\text { meinen }\end{array}$} \\
\hline & 至递 & 1 $3=$ & wert & $\mathrm{N}$ & NO & 0 & so & $\mathrm{S}$ & SW & W & NW & $\begin{array}{c}\text { Mittel- } \\
\text { wert }\end{array}$ & \\
\hline & & & & & & & & & & & & & \\
\hline
\end{tabular}

\section{Ulmus montana With. (U. seabra Mill.)}

a) untere Grenze

\begin{tabular}{|c|c|c|c|c|c|c|c|c|c|c|c|c|}
\hline summe & 4834 & 4804 & 435 & 256 & 683 & 154 & $125 \%$ & 2215 & 516 & 280 & $7 \pm 80$ & 12374 \\
\hline Anzahl & 13 & 13 & 1 & 1 & 2 & 5 & 3 & 7 & 2 & 1 & 22 & 35 \\
\hline Mittel & $3 \bar{i}+j$ & $3 \pi 6$ & 435 & 256 & 311 & $36 ! 4$ & $41 \%$ & $316 j$ & 258 & 380 & $3+0$ & $3 \tilde{5} 4$ \\
\hline Min. & 179 & & & & & & & & & & & \\
\hline
\end{tabular}

b) Baumform, obere Grenze

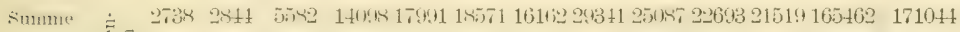

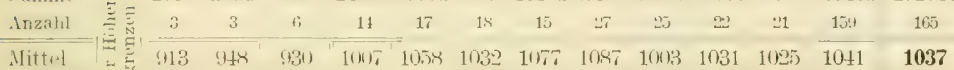

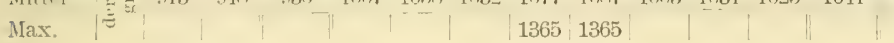

c) Strauchform, obere Grenze

\begin{tabular}{|c|c|c|c|c|c|c|}
\hline Summe & 2231 & 3865 & 1100 & 2241 & 995 & 10432 \\
\hline Alizalıl & $y$ & 3 & 1 & " & 1 & 9 \\
\hline Mittil & 1116 & 1 this & 1100 & 1121 & 995 & 1159 \\
\hline- & & & & & & \\
\hline
\end{tabular}

Tilia parrifolia Ehrh. (T. cordata Milı.)

a) Baumform, obere Grenze

\begin{tabular}{|c|c|c|c|c|c|c|c|c|c|c|c|c|c|}
\hline Summe & $\dot{z}=$ & 3876 & 3876 & 2965 & 4153 & 6428 & 5727 & 4417 & 8800 & 6261 & 2189 & 40940 & 44816 \\
\hline Anzahl & $\frac{2}{20}$ & 6 & 6 & 4 & 6 & 9 & 8 & 6 & 12 & 9 & $|3|$ & 57 & 63 \\
\hline Mittel & $=5$ & filfj & $f i f$ & 7.41 & 692 & 711 & 716 & 736 & 733 & $690^{\circ}$ & 7311 & 718 & 711 \\
\hline
\end{tabular}

b) Strauchform, obere Grenze

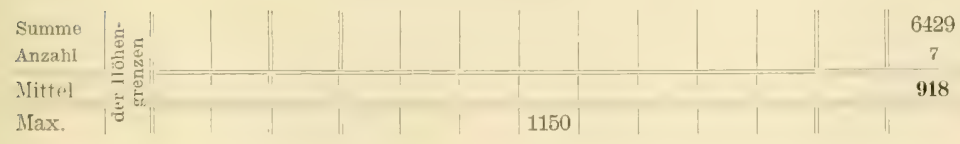




\begin{tabular}{|c|c|c|c|c|c|c|c|c|c|c|c|c|}
\hline \multirow{2}{*}{ 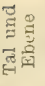 } & \multirow{2}{*}{ 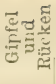 } & \multirow{2}{*}{$\begin{array}{l}\text { Mittel- } \\
\text { wert }\end{array}$} & \multicolumn{9}{|c|}{ Berglehnen nach ihrer Exposition } & \multirow{2}{*}{$\begin{array}{c}\text { Im } \\
\text { Allge- } \\
\text { meinen }\end{array}$} \\
\hline & & & $N$ & NO & 0 & So & $\mathrm{S}$ & SW & W & NW & $\begin{array}{l}\text { Mittel- } \\
\text { wert }\end{array}$ & \\
\hline
\end{tabular}

\section{Acer platanoides L.}

a) Baumform, obere Grenze

\begin{tabular}{|c|c|c|c|c|c|c|c|c|c|c|c|c|c|c|}
\hline Summe & & 7129 & 13125 & 520354 & 201807 & $2 \pi 163$ & 53.003 & 1340.1 & $20+1$ & 525231 & 1) $2381 ?$ & 13318 & 189925 & 210179 \\
\hline AnzalıI & & 8 & 13 & 21 & 20 & 26 & .33 & 211 & $3 i$ & 23 & 2 & 12 & 18 & 303 \\
\hline itte & & 891 & 1010 & 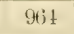 & 1010 & 1045 & 1061 & $10 \%$ & $105 \overline{7}$ & 1010 & 1037 & 1027 & 1044 & 1035 \\
\hline ax. & & & & & & & & & 1279 & & & & & \\
\hline
\end{tabular}

b) Strauchform, obere Grenze

\begin{tabular}{|c|c|c|c|c|c|c|c|c|c|c|}
\hline Summe & 2134 & 2131 & 3610 & 1210 & 12tin & $2-\pi 01$ & $610 R$ & 2470 & 17148 & 19282 \\
\hline Anzahl & 2 & 2 & 3 & 1 & 1 & 2 & 5 & $\ddot{2}$ & 14 & 1fi \\
\hline Mittel & 1067 & 10167 & 1203 & 1300 & $124 i 1$ & 1251 & 1222 & 1935 & 1225 & 1205 \\
\hline Max. & & & & & & & & 0 & & \\
\hline
\end{tabular}

\section{Acer Pseudoplatanus L.}

a) Baumform, obere Grenze

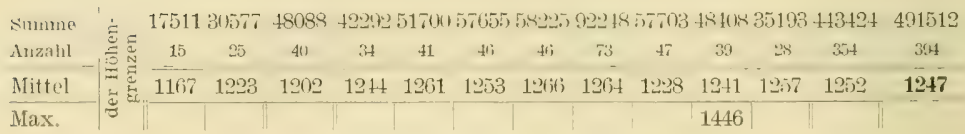

b) verkrüppelt, obere Grenze

\begin{tabular}{l|l|l|c|c|c|c|c|c|c|c|c|c}
$\begin{array}{l}\text { Summe } \\
\text { Anzahl }\end{array}$ \\
\hline Mittel \\
Max.
\end{tabular}

\section{Acer campestre L.}

a) Baumform, obere Grenze

\begin{tabular}{|c|c|c|c|c|c|c|c|c|c|c|c|c|c|}
\hline Summe & & 2933 & 1201 & 4134 & 410 & 3290 & 3646 & 3015 & 5744 & $38 \times 9$ & $75 \pi$ & $102 \pm 1$ & $233 \pi$ \\
\hline Anzahl & & 6 & 2 & 8 & 1 & 5 & 5 & 5 & 11 & 5 & 2 & 34 & 42 \\
\hline Mittel & 2 & 489 & 6010 & 517 & 400 & 6is & 52 & 603 & 522 & $67 x$ & 379 & $\pi t 66$ & $5 \overline{36}$ \\
\hline Max. & & & & & & & & 895 & & & & & \\
\hline
\end{tabular}




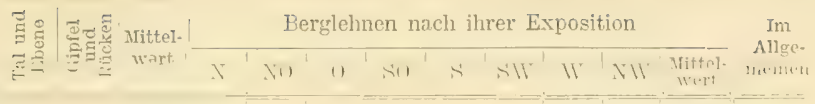

b) Strauchform, obere Grenze

\begin{tabular}{|c|c|c|c|c|c|c|c|c|c|c|c|c|}
\hline Summe & $\dot{H}$ & 752 & 857 & 1609 & 860 & 789 & 820 & 1661 & 3853 & 898 & 8881 & 10490 \\
\hline Arzahn & $\stackrel{3}{=} \frac{2}{4}$ & $\mathfrak{t}$ & 1 & 2 & 1 & 1 & 1 & $\because$ & 5 & 1 & 11 & $1: 3$ \\
\hline Mitful & $=\overrightarrow{5}$ & $\pi 2$ & $x, 4$ & 811 & (4) in & $7 x ! 1$ & 8311 & (in) & $\because 1$ & inth & (⿻1, & $80 \bar{t}$ \\
\hline Max. & $\pi$ & & & & & & & 909 & & & & \\
\hline
\end{tabular}

\section{Fraxinus excelsior I.}

a) bestandbildend, obere Grenze

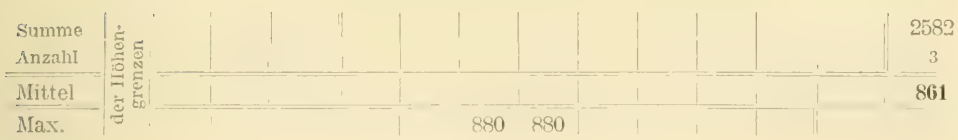

b) vereinzelt (Baunform), obere Grenze

\begin{tabular}{|c|c|c|c|c|c|c|c|c|c|c|c|c|c|c|}
\hline S"11111me* & & $8: 0$ & 4220 & $5(0,0)$ & 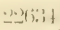 & $24,3,7$ & 3014. & shitis & Burl & $2 x-2,21$ & $330 \times i$ & 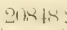 & Sull)!(i) & 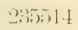 \\
\hline Anzahl & 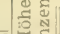 & 1 & 4 & 5 & 22 & 28 & 30 & 39 & 37 & 30 & 32 & 20 & 228 & 233 \\
\hline Mittel & 5 & $\times 30$ & {$[1, \pi)$} & 10111 & $1(1-2) \mid$ & 10113 & J111i; & $(195$ & 1115 & $1+1$ & 1181 & $111+3$ & $1+11$ & 1011 \\
\hline Max. & & & & & & & & & 1300 & & & & & \\
\hline
\end{tabular}

\section{Sorbus aucuparia $I_{\text {. }}$}

a) Baumform, obere Grenze

\begin{tabular}{|c|c|c|c|c|c|c|c|c|c|c|c|c|c|}
\hline Summe & & 2310 & 2310 & 21494 & 24551 & 19593 & 14871 & $2518 \pi$ & 19988 & $206 \pi 5$ & 20359 & 166716 & 169056 \\
\hline Anzalıl & \% & 2 & 2 & 17 & 20 & 16 & 12 & 21 & 17 & 16 & 16 & 135 & $13 \bar{\gamma}$ \\
\hline $\begin{array}{l}\text { littı. } \\
\text { Iax. }\end{array}$ & $=\bar{\vdots}$ & 1170 & 1170 & $1: 315$ & $1: 2 x$ & $1 \pm x$ & 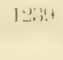 & $\begin{array}{l}1154 \\
1496\end{array}$ & $117 i$ & 1292 & 1272 & $123 \pi$ & 1234 \\
\hline
\end{tabular}

b) verkrüppelt, obere Grenze

\begin{tabular}{|c|c|c|c|c|c|c|c|c|c|c|c|c|c|}
\hline Summe & $\notin 1$ & 7205 & 7205 & $14: 61$ & 20087 & $17+93$ & 15505 & 22526 & 12948 & 2891 & 11296 & 117007 & 124212 \\
\hline Anzahl & 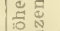 & 5 & 5 & 10 & 14 & 12 & 11 & 16 & 10 & 2 & 8 & 83 & 88 \\
\hline Mittul & $=\sum_{-1}$ & $1+11$ & $1+11$ & 1421 & $14 i n$ & $15 x$ & $1+111$ & 140 & $13 m$ & $1+45$ & $1+12$ & $1+111$ & 1412 \\
\hline Iax. & $1 \lesssim$ & & & & 1718 & 1718 & & & & & & & \\
\hline
\end{tabular}

\section{Syringa Josikaea Jaeq. fil.}

$\begin{array}{l:l}\text { Min. } & 301 \\ \text { Max. } & 568\end{array}$




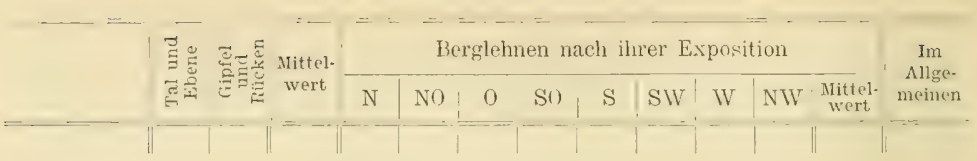

Sambucus racemosa $\mathbf{L}$.

a) untere Grenze

\begin{tabular}{|c|c|c|c|c|c|c|c|c|c|}
\hline $\begin{array}{l}\text { Summe } \\
\text { Anzahl }\end{array}$ & 邑 & $\begin{array}{c}1337 \\
3\end{array}$ & $\begin{array}{c}1337 \\
3\end{array}$ & $\begin{array}{c}522 \\
1\end{array}$ & $\begin{array}{c}1231 \\
2\end{array}$ & $\begin{array}{c}548 \\
1\end{array}$ & $\begin{array}{c}1146 \\
2\end{array}$ & $\begin{array}{c}448 \\
1\end{array}$ & $\begin{array}{c}5232 \\
10\end{array}$ \\
\hline Mittel & 5 & 416 & 416 & 522 & 616 & $5+8$ & 573 & 418 & 523 \\
\hline Min. & & 236 & & & & & & & \\
\hline
\end{tabular}

b) obere Grenze

\begin{tabular}{|c|c|c|c|c|c|c|c|c|c|c|c|c|}
\hline $\begin{array}{l}\text { Summe } \\
\text { Anzahl }\end{array}$ & & 1353 & 1353 & 2968 & 6747 & 4020 & 5199 & 1335 & $\begin{array}{c}2487 \\
2\end{array}$ & 1300 & 24056 & 25409 \\
\hline Mittel & 5 & 1353 & 1353 & 1481 & 1349 & 1340 & 1300 & 1335 & 1214 & 1300 & 1336 & 1337 \\
\hline Max. & & & & 1570 & & & & & & & & \\
\hline
\end{tabular}




\section{Ostkarpathen.}

Radnai Havasok = Radnaer Alpen (Nagy Pietrosz) 2305 m., Borgói Havasok = Borgóer Alpen (Omuluj) 1932 m., Kelemen-csoport = Kelemen-Stock (Pietroszul) 2102 m., Gyergyói Hegycsoport = Gyergyóer Gebirge (Nagy Hagymás) 1793 m., Bodzai Hegycsoport = Bodzaer Gebirge (Csukás) 1958 m., Görgényi Havasok = Görgény Gebirge (Mezóhavas) 1777 m., Hargitta C-3birge $1801 \mathrm{~m}$.

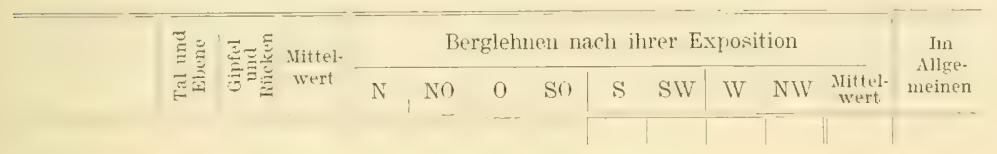

\section{Picen excelsa Lk.}

a) vereinzelt, untere Grenze

\begin{tabular}{|c|c|c|c|c|c|c|c|c|c|c|c|c|c|c|}
\hline Summe & & 12754 & 2179 & $14 ! 133$ & 3303 & $37+9$ & 1574 & 689 & 4116 & 908 & 6328 & 3735 & 26402 & 41335 \\
\hline Anzahl & 施 & $1 x$ & 2 & 20 & 5 & 4 & 6 & 1 & 6 & 1 & 8 & 5 & 36 & 56 \\
\hline Mittel & $=5$ & 709 & 1089 & 747 & (iti) & bist & 762 & (i8!) & (ixt) & 305 & 791 & 74 & 733 & 738 \\
\hline Min. & 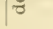 & & & & & & & & 522 & & & & & \\
\hline
\end{tabular}

b) rein, oder vorherrschend, untere Grenze

\begin{tabular}{|c|c|c|c|c|c|c|c|c|c|c|c|c|}
\hline Sumune & $\therefore$ & 24128 & $2112 x$ & $1+4,1$ & 3223 & $595 \%$ & 1814 & 2814 & $\$ 124$ & 1777 & 34561 & $580 \times 9$ \\
\hline Anzalil & $\frac{3}{2}$ & 31 & 31 & 17 & 4 & 7 & 2 & 3 & 5 & 2 & 40 & 71 \\
\hline Mlittel & $\overline{5}$ & $7 \pi 8$ & in & Sit & 806 & (x) 1 & 3197 & 138 & 825 & 888 & $x 6.4$ & 827 \\
\hline Min. & & 523 & & & & & & & & & & \\
\hline
\end{tabular}

c) geschlossener Bestand, obere Grenze

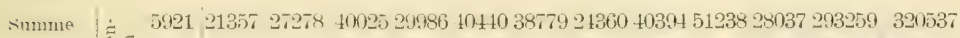

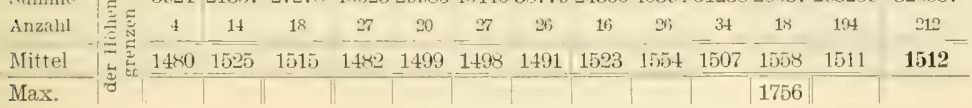

d) Raumbestand, obere Grenze (Waldgrenze)

$\begin{aligned} & \text { Sumuce } \\ & \text { Anzahl }\end{aligned}$
$\begin{aligned} & \text { Mittel } \\ & \text { Max. }\end{aligned}$




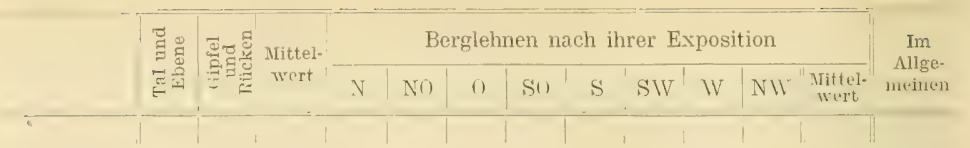

e) obere Baumgrenze

(Bäume mit $8 \mathrm{~m}$. Gipfelhöhe.)

\begin{tabular}{|c|c|c|c|c|c|c|c|c|c|c|c|c|c|c|}
\hline Summute & & (i1אij & $3: 5$ & $9+f ; 1)$ & 3217 & $1+4 j \leqslant 11$ & 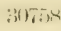 & (inisiti & (x):4i & 397. & $3=296$ & 4050 & & 30601 . \\
\hline Anzahl & 旅 & 4 & 2 & 6 & 30 & 9 & 19 & 4 & 6 & 18 & 30 & 25 & 121 & 127 \\
\hline Mittel & & $15+6$ & 1637 & $157 \%$ & $16: 11$ & $162 \div 3$ & $16 i 19$ & $16 ; 3 x$ & 16144 & $\lfloor\hat{1} \tilde{0} \mid$ & 1615 & 1620 & 1624 & 16 \\
\hline Iax. & $\pi$ & & & & 1811 & & & & & & & & & \\
\hline
\end{tabular}

f) Raumbestand mit verkriippelten Fichten, obere Grenze

\begin{tabular}{|c|c|c|c|c|c|c|c|c|c|c|c|c|}
\hline Summe & $\dot{\mu}$ & 3533 & 3533 & 1635 & 1635 & $\mid 1773$ & 5318 & 3305 & | 3526 & 11825 & 29047 & 32580 \\
\hline Anzalil & $1 \frac{2}{3} \frac{a}{2}$ & 2 & 2 & 1 & 1 & 1 & a & 3 & 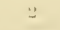 & 7 & 17 & 10 \\
\hline Mittel & $\bar{\vdots}$ & $176 t i$ & Thtit: & $|1 ;, i=|$ & 1(10ik) & 1783 & $17 \times 3$ & 11 i. & $17+i 3$ & $16 \times 3$ & 1709 & 1715 \\
\hline Miı. & & & & & & & 1874 & & & & & \\
\hline
\end{tabular}

g) verkrüppelt, obere Grenze

\begin{tabular}{|c|c|c|c|c|c|c|c|c|c|c|c|c|}
\hline Summe & & 1918 & 1918 & 5993 & 7713 & 3785 & 7901 & 9703 & ป898 & 3921 & 44817 & 46765 \\
\hline Anzahl & 起 & 1 & 1 & 3 & 4 & 2 & 4 & 5 & 3 & 2 & 23 & 24 \\
\hline Mittel & $\begin{array}{ll}\text { दि } \\
5 \\
5\end{array}$ & 1918 & ITIR & IIns & 1028 & 1893 & 19.5 & 1911 & 1943 & 1962 & $15 \% 0$ & $19+9$ \\
\hline $\operatorname{Max}$ & & & & & & & 2026 & & & & & \\
\hline
\end{tabular}

\section{Abies peetimata DC. (A. alba Mill.)}

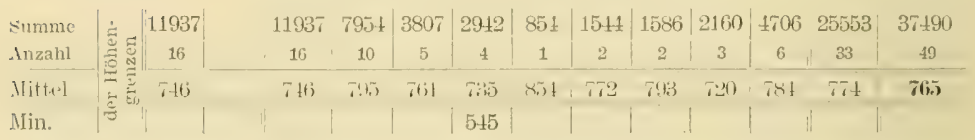

b) rein, oder vorherrschend, untere Grenze

\begin{tabular}{|c|c|c|c|c|c|c|c|c|c|c|c|}
\hline Summe & & 3923 & 3923 & 3199 & 1683 & 783 & 1633 & 1600 & 1575 & 10473 & $14390^{\circ}$ \\
\hline Anzahl & $\div 0$ & 5 & 5 & 4 & 2 & 1 & 2 & 3 & 2 & 13 & 18 \\
\hline Mlittel & 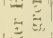 & Twi & 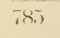 & (2010) & $x+1$ & $7 \times 3$ & 817 & 8011 & This & 806 & 800 \\
\hline lin. & एव & & - & 752 & & & & & & - & \\
\hline
\end{tabular}

c) reín, oder vorherrschend, obere Grenze

\begin{tabular}{|c|c|c|c|c|c|c|c|c|c|c|c|c|}
\hline Sumine & & 3924 & 3924 & 2627 & $2 \pm 13$ & 2569 & 2674 & 2781 & 3813 & 5136 & 22043 & 25967 \\
\hline Anzalil & & 3 & : & $\ddot{2}$ & 2 & 2 & $\because$ & 2 & 3 & + & 17 & 20 \\
\hline Mitt.el & $-=-\frac{1}{2}$ & $130 x$ & $13 x k$ & 1331:3 & $1: \%$ & $128 t$ & linis & 1390 & $13 \times 1$ & $13 \times 4$ & $12+7$ & 1208 \\
\hline Mfax. & & & & & & & & & $14 \tilde{6} 6$ & & & \\
\hline
\end{tabular}




\begin{tabular}{|c|c|c|c|c|c|c|c|c|c|c|c|c|}
\hline \multirow{2}{*}{ 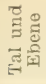 } & \multirow{2}{*}{ 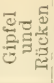 } & \multirow{2}{*}{$\begin{array}{l}\text { Mittel- } \\
\text { wert }\end{array}$} & \multicolumn{9}{|c|}{ Berglehnen nach ihrer Exposition } & \multirow{2}{*}{$\begin{array}{c}\text { Im } \\
\text { Allge- } \\
\text { meinen }\end{array}$} \\
\hline & & & $\mathrm{N}$ & NO & 0 & $\mathrm{SO}$ & $\mathrm{S}$ & STV & W & NWV & $\begin{array}{l}\text { Mittel- } \\
\text { wert }\end{array}$ & \\
\hline
\end{tabular}

d) vereinzelt (Baumform), obere Grenze

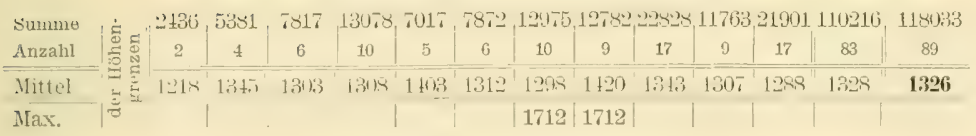

e) verkrüppelt, obere Grenze

\begin{tabular}{|c|c|c|c|c|c|c|c|c|c|c|}
\hline Summe & 1. & 1330 & 4289 & 5619 & 2918 & 3088 & 4564 & 2974 & $135+1$ & 19163 \\
\hline Aruzalıl & $\frac{3}{3}$ & 1 & 3 & 4 & 2 & 2 & $\therefore$ & 2 & 9 & 13 \\
\hline Mittel & E & 1230 & $1+30$ & $110 \pi$ & 1.4 .79 & $1.51 t^{1}$ & 1521 & 1487 & $1 \tilde{\pi} 0 \pi$ & 1474 \\
\hline Max. & $=\left.\right|^{0}$ & & & & & 1681 & & & & \\
\hline
\end{tabular}

Pinus Cembra L.

a) untere Grenze

\begin{tabular}{|c|c|c|c|c|c|c|}
\hline Summe & d. 1543 & $6036 \mid$ & 2878 & 1458 & 2992 & 14907 \\
\hline Anzahl & 융 1 & 11 & 2 & 1 & 2 & 10 \\
\hline Mittel & 1543 & $15199^{\prime}$ & 1439 & 1458 & 1496 & $1+91$ \\
\hline Min. & & 1375 & 1375 & & & \\
\hline
\end{tabular}

b) Baumform, obere Grenze

\begin{tabular}{|c|c|c|c|c|c|c|c|}
\hline Summe & & 6725 & 1708 & 1734 & 3385 & 8587 & 22139 \\
\hline Anzahl & 동 & 4 & 1 & 1 & 2 & 5 & 13 \\
\hline Mittel & 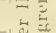 & 1681 & $17 \mathrm{kn}$ & 1734 & 1642 & 1.17 & 1703 \\
\hline Max. & & & & & & 1771 & \\
\hline
\end{tabular}

c) verkrüppelt, obere Grenze

\begin{tabular}{l|l|c|c|c|c|c|c|c|}
$\begin{array}{l}\text { Summe } \\
\text { Anzahl }\end{array}$ \\
\hline Mittel \\
\hline Max.
\end{tabular}

\section{Pinus montana Mill.}

a) sporadisch, untere Grenze

\begin{tabular}{|c|c|c|c|c|c|c|c|c|c|c|c|c|}
\hline summe & 6050 & 1.32 & 10753 & $93 \pm 0$ & $65 \pm 7$ & 6526 & 3319 & 2881 & 7695 & $317 t$ & 30115 & 50197 \\
\hline Anzahl & 4 & 3 & 7 & 6 & 4 & 4 & 2 & 2 & 5 & 2 & 25 & 32 \\
\hline Mittel & $=1.12$ & $15 \pi$ & $1 \leqslant 40$ & 1ist" & 1637 & 1631 & 165. & $1+42$ & 1539 & 159. & \multirow[t]{2}{*}{157} & \multirow[t]{2}{*}{1569} \\
\hline Min. & 0 & & & & & & & & 1328 & & & \\
\hline
\end{tabular}




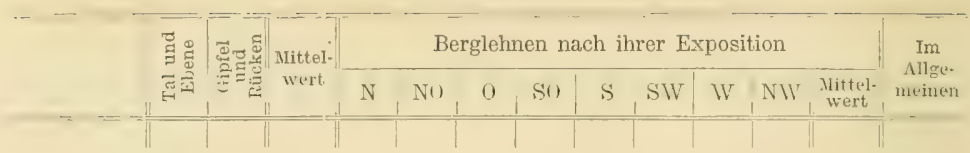

b) massenhaft, untere Grenze

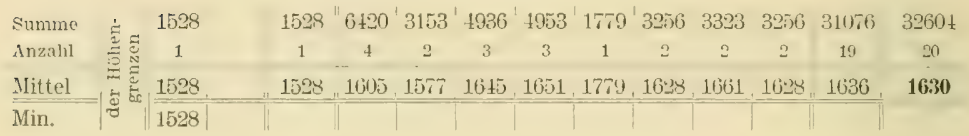

c) massenhaft, obere Grenze

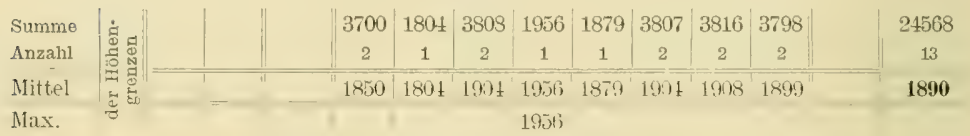

d) sporadisch, obere Grenze

\begin{tabular}{|c|c|c|c|c|c|c|c|c|c|c|c|c|c|}
\hline Summe & & 5968 & 5968 & 5697 & 5704 & 3004 & 3836 & 6113 & 4140 & 2147 & 7719 & 37361 & $4333^{\circ}$ \\
\hline Anzahl & & 3 & 3 & 3 & 3 & 1 & 2 & 3 & 2 & 1 & 4 & 19 & 22 \\
\hline Mittel & $=E$ & 1959 & $19 \nvdash 9$ & 1899 & 1901 & 20118 & 1918 & 2031 & 3070 & 2117 & 1939 & $196 i 7$ & 1970 \\
\hline Max. & 1 & & & & & & & & & $21 \pm$ & 2147 & & \\
\hline
\end{tabular}

\section{Jumiperus communis $\mathbf{L}$.}

\begin{tabular}{|c|c|c|c|c|c|c|c|c|c|c|}
\hline Summe & & 12503 & 3273 & $15 \geq 1$ & 40.93 & $14 \pm 6$ & 7370 & 5.21 & 2759 & 38169 \\
\hline Anzalıl & |气 & 9 & 3 & 1 & 3 & 1 & 5 & 4 & 2 & 28 \\
\hline $\begin{array}{l}\text { Mittel } \\
\text { Max. }\end{array}$ & | & 1389 & 1091 & $\begin{array}{l}1521 \\
1521\end{array}$ & 1361 & $1+26$ & 1474 & 1300 & 1379 & 1363 \\
\hline
\end{tabular}

\section{Juniperus nana Willd.}

a) sporadisch, untere Grenze

\begin{tabular}{|c|c|c|c|c|c|c|c|c|c|c|c|c|c|c|}
\hline Summe & & 9698 & 1003: & 19730 & 3504 & $7+32$ & 6795 & 5,66 & 7105 & 6397 & $93 \pm 7$ & 3819 & 50791 & 70524 \\
\hline Anzahl & & 8 & 8 & 16 & 3 & 5 & 5 & 4 & fi & 5 & 7 & 3 & 28 & 54 \\
\hline Mittel & 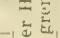 & 1212 & 1251 & 1233 & 103 is & $1+56$ & 1354 & $1+41$ & $123 t$ & $1 \div 79$ & $133 i$ & $12 \times 3$ & 1337 & 1306 \\
\hline Min. & 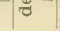 & & 775 & & & & & & & & & & & \\
\hline
\end{tabular}




\begin{tabular}{|c|c|c|c|c|c|c|c|c|c|c|c|c|}
\hline \multirow{2}{*}{ 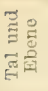 } & \multirow{2}{*}{ 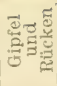 } & \multirow{2}{*}{$\begin{array}{l}\text { Mittel- } \\
\text { wert }\end{array}$} & \multicolumn{9}{|c|}{ Berglehnen nach ihrer Exposition } & \multirow{2}{*}{$\begin{array}{c}\text { Im } \\
\text { Allge. } \\
\text { meinen }\end{array}$} \\
\hline & & & $\mathrm{N}$ & NO & 0 & SO & $\mathrm{S}$ & SW & WV & NW & $\begin{array}{l}\text { Mittel- } \\
\text { wert }\end{array}$ & \\
\hline
\end{tabular}

b) massenhaft, untere Grenze

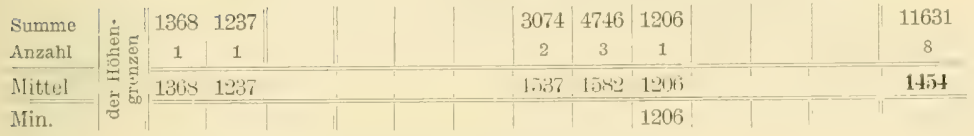

c) massenhaft, obere Grenze

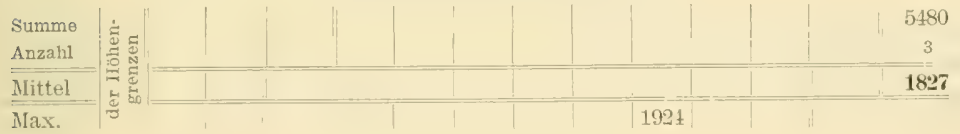

d) sporadisch, obere Grenze

\begin{tabular}{|c|c|c|c|c|c|c|c|}
\hline Summe & 3978 & 2011 & 4058 & 4022 & 4000 & 1892 & 19961 \\
\hline Anzahl & 2 & 1 & 2 & 2 & 2 & 1 & 10 \\
\hline Mittel & 1989 & 3011 & 3020 & 3011 & 3000 & 1892 & 1996 \\
\hline Max. & & & 2140 & & & & \\
\hline
\end{tabular}

\section{Fagus silvatica L. $^{*}$}

a) Bestandbildung im Allgemeinen, obere Grenze

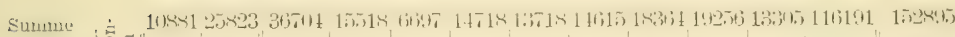

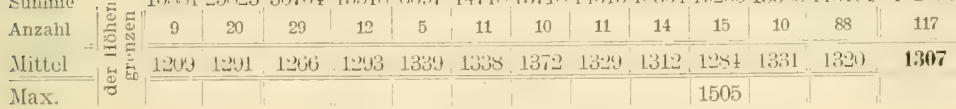

$a_{1}$ ) obere Grenze der Bestandbildung, als Waldgrenze

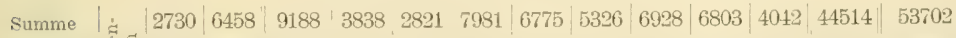

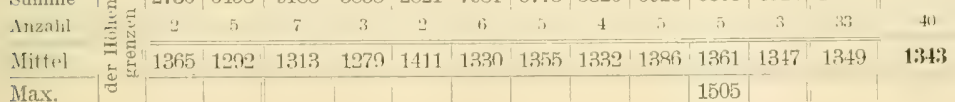

$a_{2}$ ) obere Grenze der Bestandbildung, darüber Fichte

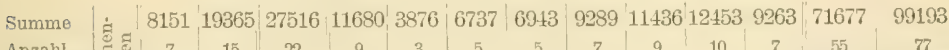
\begin{tabular}{l|c|c|c|c|cc|c|c|c|c|c|c} 
Anzah & Mittel \\
M
\end{tabular} Max. $\mid$ M $|1490|$

* Erreicht ihre untere Grenze im Siebenbürgischen Becken. 


\begin{tabular}{|c|c|c|c|c|c|c|c|c|c|c|c|}
\hline & 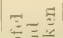 & vit $t \cdot \cdot \cdot$. & \multicolumn{8}{|c|}{ Berglehnen nach ihrer Exposition } & Inı \\
\hline त्रा & $\vec{b}=\overrightarrow{4}$ & wert & $\mathrm{N}$ & NO & 0 & $\mathrm{SO}, \mathrm{S}$ & SW & W & $\mathrm{NW}$ & $\begin{array}{l}\text { Mittel- } \\
\text { wert }\end{array}$ & meinen \\
\hline
\end{tabular}

b) sporadisches Vorkommen im Fichtenbestand, obere Grenze

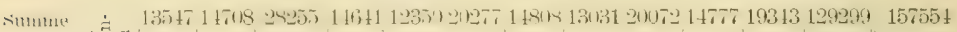

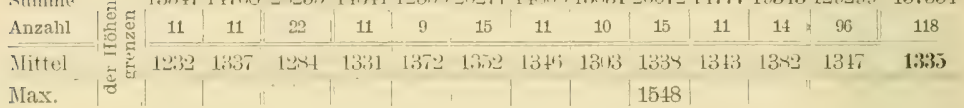

c) Strauchform, obere Grenze

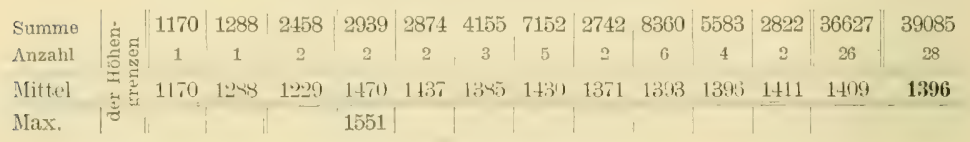

\section{Quereus sessilillora Salisb.}

a) rein, oder vorherrschend, obere Grenze

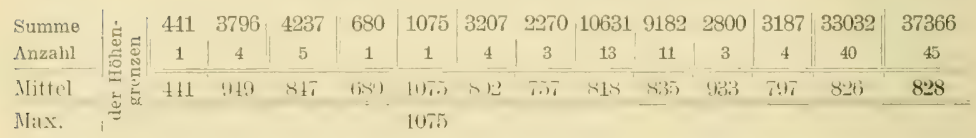

b) rereinzelt (Baumform), obere Grenze

\begin{tabular}{|c|c|c|c|c|c|c|c|c|c|c|c|c|c|c|}
\hline Summe & & 2250 & 2588 & 4838 & 1821 & 2134 & 2788 & 5547 & 11215 & 11709 & 6856 & 2003 & 44073 & 48911 \\
\hline Anzahl & $\mid$\begin{tabular}{ll}
9 & 5 \\
\hdashline & 0 \\
0 & 0
\end{tabular} & 3 & 3 & 6 & 2 & 2 & 3 & 6 & 12 & 12 & 7 & 2 & 46 & 52 \\
\hline Mitter & I들 & $7 \pi 0$ & 863 & sens & 911 & 1067 & 929 & 925 & 875 & 976 & 979 & 1002 & 9,8 & 941 \\
\hline Max. & & & & & & 1120 & & & & & & & & \\
\hline
\end{tabular}

c) verkrüppelt, obere Grenze

\begin{tabular}{|c|c|c|c|c|c|c|}
\hline $\begin{array}{l}\text { Summe } \\
\text { Anzahl }\end{array}$ & $\begin{array}{c}1170 \\
1\end{array}$ & $\begin{array}{c}972 \\
1\end{array}$ & $\begin{array}{c}1015 \\
1\end{array}$ & $\begin{array}{c}7463 \\
7\end{array}$ & $\begin{array}{c}2887 \\
3\end{array}$ & $\begin{array}{c}13507 \\
13\end{array}$ \\
\hline Mittel & 1170 & $4 \pi^{2}-2$ & 1015 & 101iij & 90 & 1039 \\
\hline Max. & & & & 1336 & & \\
\hline
\end{tabular}

Querens pedunculata Eloh. (Qu. Robur L.)

a) rein, oder vorherrschend, obere Grenze

\begin{tabular}{|c|c|c|c|c|c|}
\hline Summe & 583 & 700 & 520 & 1284 & $\begin{array}{c}3087 \\
5\end{array}$ \\
\hline Mittel & च & & & & 617 \\
\hline Max. & & 700 & & & \\
\hline
\end{tabular}




\begin{tabular}{|c|c|c|c|c|c|c|c|c|c|c|c|c|}
\hline \multirow{2}{*}{ 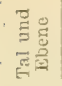 } & \multirow{2}{*}{ 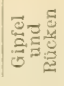 } & \multirow{2}{*}{$\begin{array}{l}\text { Mittel- } \\
\text { wert }\end{array}$} & \multicolumn{9}{|c|}{ Berglehnen nach ihrer Exposition } & \multirow{2}{*}{$\begin{array}{c}\text { Im } \\
\text { Allge- } \\
\text { meiner }\end{array}$} \\
\hline & & & $N$ & NO & 0 & SO & $\mathrm{S}$ & SWV & WV & NIV & & \\
\hline
\end{tabular}

b) vereinzelt (Baumform), obere Grenze

\begin{tabular}{|c|c|c|c|c|c|c|c|c|c|c|}
\hline Summo & 4116 & 4416 & 2031 & 3005 & 5412 & 6836 & 1725 & 2786 & 21795 & 26211 \\
\hline Anzahl & 6 & 6 & 3 & 4 & 7 & 8 & 2 & 4 & 28 & 34 \\
\hline Mittel & $-i 3 i$ & $7: 36$ & fiT. & i.il & 773 & xit & Sit) & $6 \%$ & 7.8 & 771 \\
\hline Max. & & & & । & & 993 & & & & \\
\hline
\end{tabular}

c) verkrüppelt, obere Grenze

\begin{tabular}{|c|c|c|c|c|}
\hline summe & \multirow{4}{*}{ 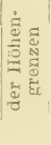 } & 1015 & $927 \mid 978$ & 2930 \\
\hline Anzahl & & 1 & 111 & 3 \\
\hline Mittel & & & & 973 \\
\hline Max. & & $\begin{array}{l}1015 \\
.\end{array}$ & & \\
\hline
\end{tabular}

Carpinus Betulus I.

a) bestandbildend, obere Grenze

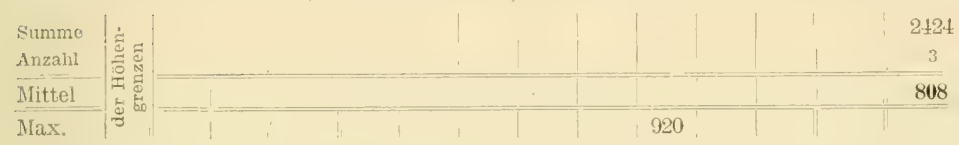

b) vereinzelt (Baumform), obere Grenze

\begin{tabular}{|c|c|c|c|c|c|c|c|c|c|c|c|c|c|c|}
\hline Summe & & 10856 & 2769 & 13625 & 3314 & 3576 & 6059 & 2811 & 6238 & 7657 & 2639 & 3830 & $3612 \pm$ & 49749 \\
\hline Anzahl & $\frac{\stackrel{\Xi}{\Xi}}{\square}$ & 13 & 3 & 16 & 4 & 4 & 7 & 3 & 7 & 9 & 3 & 4 & $4 \mathrm{~L}$ & 57 \\
\hline Mittin] & $=$ & $x, \ldots ;$ & 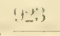 & $\therefore i 2$ & $4 \%$ & $x+1$ & siti & 43 & .11 & -i1 & $x+1$ & 56 & $+<1$ & $87: 3$ \\
\hline Max. & & & & & & & 1172 & 1172 & & & & & & \\
\hline
\end{tabular}

c) Strauchform, obere Grenze

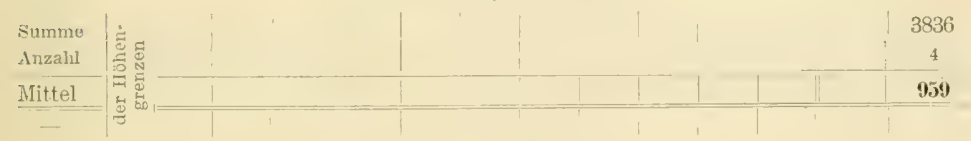

\section{Corylus Avellana 1.}

obere Grenzo

\begin{tabular}{|c|c|c|c|c|c|c|c|c|c|c|c|c|c|c|}
\hline Summe & & 7817 & 11237 & 19084 & 3311 & 4479 & 10172 & 6685 & 11761 & 9351 & 2217 & 6904 & 55180 & 74261 \\
\hline Inzahl & 宩 & 8 & $\begin{array}{l}9 \\
\end{array}$ & 17 & 3 & 4 & 9 & 5 & 10 & 8 & 2 & 6 & 47 & 64 \\
\hline Mittil. & 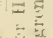 & SKI & 1219 & 1133 & $1111 \mathrm{t}$ & 11311 & 11 tit & 1:i.i. & $11 \pi$ & 1103.4 & 1111,4 & 1151 & 1171 & $111 \% 0$ \\
\hline Max. & $\approx$ & & & & & & & 1528 & & & & & & \\
\hline
\end{tabular}




\begin{tabular}{|c|c|c|c|c|c|c|c|c|c|c|c|c|c|}
\hline "ॄ̊ & & & \multicolumn{10}{|c|}{ Berglehnen nach ihrer Exposition } & \multirow{2}{*}{$\begin{array}{c}\text { Im } \\
\text { Allgo* } \\
\text { meinen }\end{array}$} \\
\hline สํำ| & $\mathrm{S}^{5}$ & & & NO & ( & & S & STV & W & N & $\|$ & $\begin{array}{l}\text { fittel- } \\
\text { wert }\end{array}$ & \\
\hline
\end{tabular}

\section{Betula verrucosa Ehrh.}

a) bestandbildend, obere Grenze

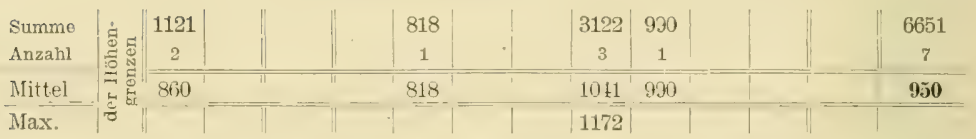

b) vereinzelt (Baumform), obere Grenze

\begin{tabular}{|c|c|c|c|c|c|c|c|c|c|c|c|c|c|c|}
\hline Summe & & 1300 & 7188 & 8488 & 5185 & 6161 & 4003 & 5038 & 10017 & 7658 & 6122 & $6 \geq 99$ & 50196 & 58984 \\
\hline Anzah & & 1 & 5 & 6 & 4 & 5 & 3 & 4 & 8 & 6 & 5 & 5 & 40 & th \\
\hline littel & & 1300 & 1438 & $141 \tilde{5}$ & 1396 & 1232 & 1331. & 1265 & 1252 & 1275 & 1224 & 1260 & 12152 & 1282 \\
\hline Max. & & & & & & & & & 1610 & & & & & \\
\hline
\end{tabular}

\section{Alnus glutinosa Gärtu.}

a) massenhaft, obere Grenze

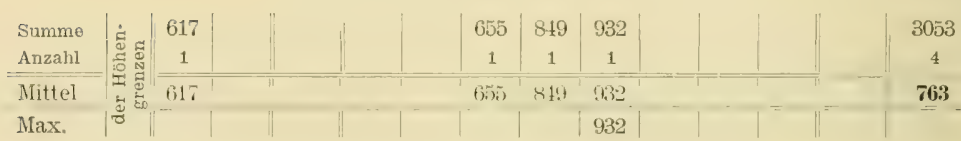

b) vereinzelt, obere Grenze

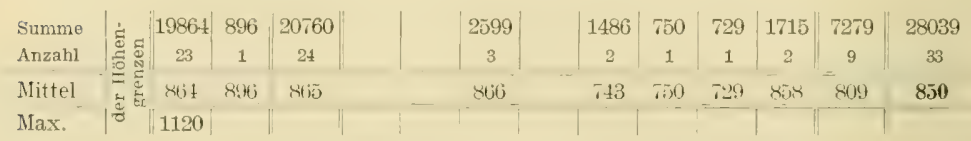

\section{Alnus incana Willd.}

a) Baumform, obere Grenze

\begin{tabular}{l|c|c|c|c|c|c|c|c|c|c|c}
$\begin{array}{c}\text { Summe } \\
\text { Anzahl }\end{array}$ \\
\hline Mittel
\end{tabular}




\begin{tabular}{|c|c|c|c|c|c|c|c|c|}
\hline$\Rightarrow \approx$ & & & glehnen ne & $\operatorname{ch~} \mathrm{i}$ & rer $\mathrm{E}$. & position & & $\operatorname{Im}$ \\
\hline 190 & wert & $N \quad x$ & 0 si & $S$ & SII & IV NW & $\begin{array}{c}\text { Mittel- } \\
\text { wert }\end{array}$ & meinen \\
\hline
\end{tabular}

b) Strauchform, obere Grenze

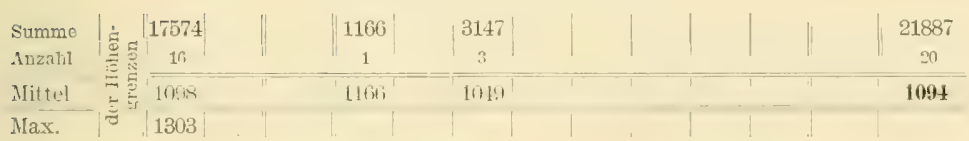

\section{Alums riridis $0 \mathrm{C}$.}

a) untere Grenze

\begin{tabular}{|c|c|c|c|c|c|c|c|c|c|c|}
\hline Sunme & 10835 & 10835 & 6385 & $3: 81$ & +200 & 2456 & 2608 & $43 \pm$ & 23890 & 34.25 \\
\hline Anzahl & 10 & 10 & 5 & 3 & 3 & 2 & 2 & 3 & 18 & 28 \\
\hline Mittel & $10 \times 3$ & 1083 & 126 & $10 \div 4$ & 1.419 & $14 \pi$ & 1341 & $14 \pi$ & 1327 & 1240 \\
\hline Min. & 6.7 & & & & & & & & & \\
\hline
\end{tabular}

b) obere Grenze

\begin{tabular}{|c|c|c|c|c|c|c|c|c|c|c|c|c|}
\hline Summe & 1619 & 1827 & 3446 & 5197 & 1820 & 5529 & 1768 & 3824 & 3816 & 3693 & 25677 & 29123 \\
\hline Anzahl & 1 & 1 & 2 & 3 & 1 & 3 & 1 & 2 & 3 & 2 & 14 & 16 \\
\hline Mittel & 1619 & $1 \times 2-7$ & 1723 & 1732 & $1 \times=0$ & $1 \times 43$ & 1.tix & 1912 & 1433 & $1 \times 4 k$ & $1 \times 31$ & $18: 0$ \\
\hline Max. & & & & & & 2004 & & & & & & \\
\hline
\end{tabular}

\section{Salix pentandra L.}

obere Grenze

\begin{tabular}{|c|c|c|c|c|c|c|c|}
\hline Summe & 10688 & 1170 & 6541 & 2122 & 3525 & 1192 & 25238 \\
\hline Anzahl & 10 & 1 & 6 & 2 & 3 & 1 & 23 \\
\hline Mittel & $100 \%$ & $11 \% 0$ & 1090 & 1061 & 1175 & $11 \div 2$ & 1097 \\
\hline Max. & & & 1287 & & & & \\
\hline
\end{tabular}

\section{Populus tremula $\mathbf{L}$.}

a) Baumform, obere Grenze

\begin{tabular}{|c|c|c|c|c|c|c|c|c|c|c|c|c|c|c|}
\hline Sumrne & & 1143 & $6+12$ & $108 \tilde{\text { กิ }}$ & 1205 & 46.38 & 21.78 & 3201 & $343 t$ & $139 \%$ & 3402 & 1119 & 21032 & $31 \times x=$ \\
\hline Anzalıl & & 4 & 5 & 9 & 1 & 4 & 2 & 3 & 3 & 1 & 3 & 1 & 18 & 27 \\
\hline D. Ilittel & & 1111 & 1:34? & $1: 31 \times j$ & $12(5)$ & 1160 & 1226 & $100 \mathrm{~K}$ & $11+5$ & $13 ! 7$ & 1134 & 1119 & 1108 & 1181 \\
\hline Max. & & & & & & & & & & 1397 & & & & \\
\hline
\end{tabular}




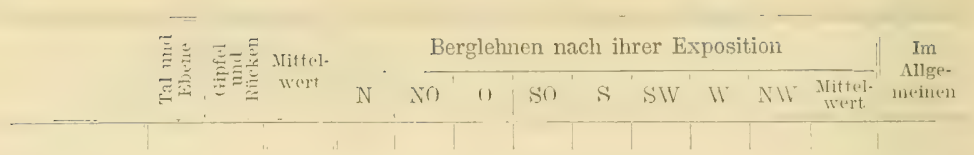

b) Stranchform, obere Grenze

\begin{tabular}{|c|c|c|c|c|c|c|c|}
\hline Summe & 1132 & 2868 & 1468 & 3182 & 1398 & 1387 & $1143 \bar{\jmath}$ \\
\hline Anzalnl & $\mid \begin{array}{l}\mid \\
1\end{array}$ & 2 & 1 & 2 & 1 & 1 & 8 \\
\hline Mittel & 1132 & 1434 & 14 th & $1 \operatorname{lin} 1$ & 1398 & 1387 & 1429 \\
\hline Max. & & & & 1637 & & & \\
\hline
\end{tabular}

Ulmus montana With. (U. seabra Mill.)

a) untere Grenze

\begin{tabular}{|c|c|c|c|c|c|c|c|c|c|}
\hline summo & 1392 & is 1 & $13 \pm 3$ & 1725 & $8 \pm 3$ & 1420 & 1180 & 2189 & $10 \pi 3$ \\
\hline Anzalil & 2 & 1 & 2 & 2 & 1 & 2 & 2 & 3 & 15 \\
\hline Mittel & 696 & inl & ifil & MiL & $x+3$ & 710 & $\sin (x)$ & 730 & 717 \\
\hline Min. & & & & & & & 478 & & \\
\hline
\end{tabular}

b) obere Grenze

\begin{tabular}{|c|c|c|c|c|c|c|c|c|c|c|c|c|c|c|}
\hline sиmиนи & & היהבי111 & 1088 & $1131 \mathrm{i}$ & 1.+5!11 & $(i \overline{4}) \overline{4}$ & .715 .11 & :2011 & 7 & xain & 10033 & $4 \times 13$ & 25605 & 700.5 \\
\hline Anzahl & & 9 & 1 & 10 & 12 & 6 & 6 & 3 & 6 & 7 & 5 & 4 & 49 & 59 \\
\hline Mittel & & 11.41 & $10 \mathrm{~s} x$ & $113 t$ & 1911 & $11+1$ & $11-\mathrm{s}$ & 111 ix & 120 & 1166 & 1207 & 1203 & $11 ! 18$ & 1187 \\
\hline Iax. & & & & & 1440 & & & & & & & & & \\
\hline
\end{tabular}

\section{Acer platamoides $\mathbf{L}$.}

Baumform, obere Grenze

\begin{tabular}{|c|c|c|c|c|c|c|c|c|c|c|c|}
\hline Summe & & $1+40$ & $71 \times 3$ & $3: 341$ & 流1 & $3=0$ & $433^{\circ}$ & $33 \div$ & +183 & $356 ! 113$ & 35696 \\
\hline Anzahl & 䖨 & 6 & 6 & 2 & 3 & 3 & 4 & $|3|$ & 4 & 31 & 31 \\
\hline Mittel & $=$ & 11.3 & $11: 4$ & 1145 & 1250 & 1093 & 1118 & 1118 & 1121 & 1151 & 1151 \\
\hline $\bar{M}$ Mx. & & & & & | 1381 & & & & & & \\
\hline
\end{tabular}

Acer Pseudoplatanus L.

a) Baumform, obere Grenze

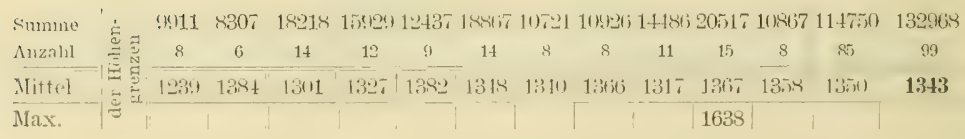




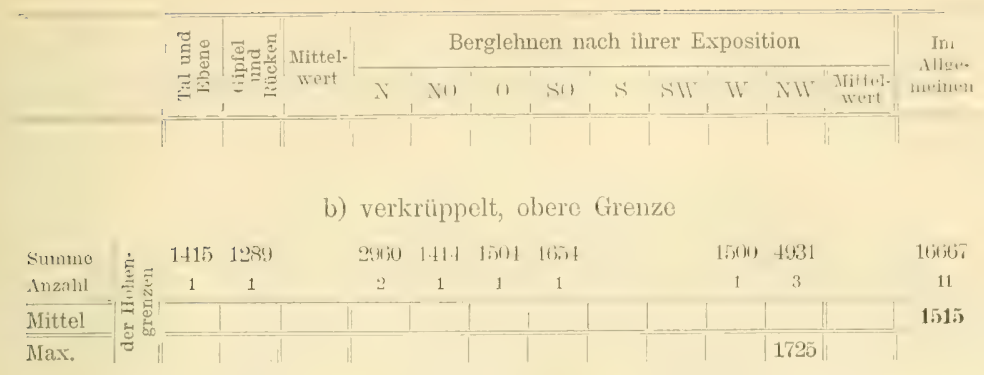

\section{Acer campestre 1 .}

obere Grenze

\begin{tabular}{|c|c|c|c|c|c|c|c|c|c|c|}
\hline Summe & 2887 & 1895 & 1590 & 1790 & 916 & 4508 & 1584 & 920 & 920 & 17040 \\
\hline Anzahl & 4 & 2 & 2 & 2 & 1 & 6 & 2 & 1 & 1 & 21 \\
\hline Mittel & & & & & & & & & & 811 \\
\hline
\end{tabular}

\section{Fraximus excelsior l.}

vereinzelt (Baumform), obere Grenze

Summe $\mid \begin{gathered}\text { S. } \\ \text { Sum }\end{gathered}$

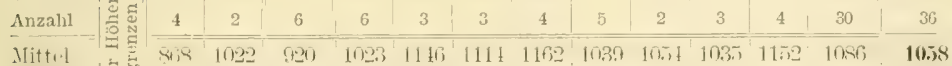

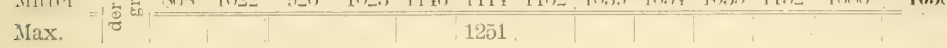

\section{Sorbus aucuparia $\mathbf{L}$.}

a) Baumform, obere Grenze

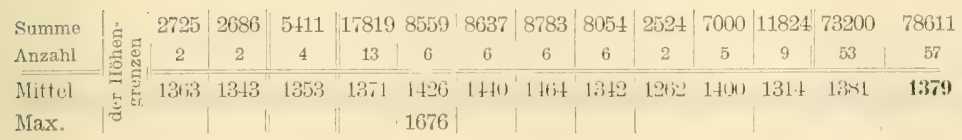

b) Strauchform, obere Grenze

\begin{tabular}{|c|c|c|c|c|c|c|c|c|c|c|c|c|c|c|}
\hline Sumune & & $15: 4$ & $33 \div 4$ & $40.60^{2}$ & $7(\pi) 3$ & $5+63$ & finint & $(i t))^{2}$ & $+t i x \mid$ & 1490 & 1.01 & $3(11)$ & $37 \times 30$ & t:ant \\
\hline Anzahl & 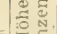 & 1 & 2 & 3 & 5 & 4 & 4 & 4 & 3 & 1 & 1 & 2 & 24 & 27 \\
\hline Mittel & $\vec{E}$ & 1007 & $16 \mathrm{f}, \mathrm{t}$ & 1612 & 1.51 & $119 \pi$ & 1611, & $16,0)$ & $118 \bar{i}$ & $11 \% 1$ & 1 तil & $\operatorname{lin} 01$ & 1.76 & 1584 \\
\hline Max. & $\approx$ & & & & & & 1841 & & & & & & & \\
\hline
\end{tabular}




\begin{tabular}{|c|c|c|c|c|c|c|c|c|c|c|c|c|c|c|c|}
\hline \multirow{2}{*}{ 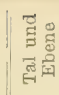 } & \multirow{2}{*}{\multicolumn{2}{|c|}{ 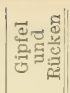 }} & \multirow{2}{*}{$\begin{array}{c}\text { Mittel- } \\
\text { wert }\end{array}$} & \multicolumn{11}{|c|}{ Berglehnen nach ihrer Exposition } & \multirow{2}{*}{$\begin{array}{c}\text { Im } \\
\text { Allge- } \\
\text { meinen }\end{array}$} \\
\hline & & & & $\mathrm{N}$ & & No & 0 & SO & $\mathrm{S}$ & SW & W & & & \begin{tabular}{|} 
Mittel- \\
wert
\end{tabular} & \\
\hline
\end{tabular}

\section{Crataegus monogyua Jacq. und oxyacantha L.}

\begin{tabular}{|c|c|c|c|c|c|c|c|c|c|c|c|}
\hline Summe & & 1687 & 1075 & 2762 & 1991 & 2968 & $\mid 9079$ & $|2836|$ & 2225 & 19102 & 21864 \\
\hline Anzahl & : 댐 & 2 & 1 & 3 & 2 & 3 & 8 & 3 & 2 & 18 & 21 \\
\hline Mittel & 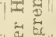 & 813 & 1075 & 921 & 997 & 289 & $113 \pi$ & 915 & 1112 & 10651 & 1041 \\
\hline Max. & $\because$ & & & & & & 1329 & & & & \\
\hline
\end{tabular}

\section{Sambucus racemosa $\mathbf{I}$.}

a) untere Grenze

Min. | | 630

b) obere Grenze

\begin{tabular}{|c|c|c|c|c|c|c|c|c|c|c|c|c|c|c|}
\hline Summe & & 4266 & 6014 & 10280 & 7266 & 4261 & 4537 & 2846 & 5728 & 1527 & 1447 & 1609 & 29221 & 39501 \\
\hline Anza & & 3 & 4 & 7 & 5 & 3 & 3 & 2 & 4 & 1 & 1 & 1 & 20 & 27 \\
\hline Mlitt $x]$ & & 1422 & 15003 & 1469 & 1428 & 1131 & 151 & 1128 & $1+32$ & 1524 & $1+4$ & lotos? & 1461 & 1463 \\
\hline Max. & & & 17.74 & & & & & & & & . & & & \\
\hline
\end{tabular}




\section{Siidkarpathen.}

Fogarasi Havasok $=$ Fogaraser Crebirge $($ Negoj) $2536 \mathrm{~m}$., Pareng (Mundra) $2520 \mathrm{~m}$., Bucsees (La Omu) $2508 \mathrm{~m}$, Retyezát (Vrf. Peleaga) $2506 \mathrm{~m}$., Szebeni vagy szászsebesi Havasok = Szebener oder Szászsebeser Gebirge (Cindrel) $2248 \mathrm{~m}$.

\begin{tabular}{|c|c|c|c|c|c|c|c|c|c|}
\hline \multirow{2}{*}{ 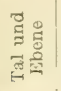 } & \multirow{2}{*}{ 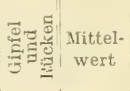 } & \multicolumn{7}{|c|}{ Berglehmen nach ihrer Exposition } & \multirow{2}{*}{$\begin{array}{l}\text { Im } \\
\text { Allge- } \\
\text { meiner }\end{array}$} \\
\hline & & $\mathrm{Nol}$ & $n^{\prime} \mathrm{s} \cap$ & $\mathrm{S}$ & SW & IV & NW & $\begin{array}{l}\text { Mittel- } \\
\text { wert }\end{array}$ & \\
\hline
\end{tabular}

\section{Picea excelsa Lk.}

a) vereinzelt, untere Grenze

\begin{tabular}{|c|c|c|c|c|c|c|c|c|c|c|c|c|c|c|}
\hline Summe & & $8+i 80$ & $11: 0$ & 9800 & 11815 & 3027 & tais & (i20) & 1titio & 1398 & 4976 & 3092 & 31612 & 41410 \\
\hline Anzahl & 10ี & 11 & 1 & 12 & 16 & 4 & 6 & 1 & 2 & 2 & 6 & 3 & 40 & 52 \\
\hline Mittel & 出 & 789 & 1120 & 817 & -38 & 807 & $826)$ & (i3) & $\times 33$ & fitit & 829 & 1007 & 790 & 796 \\
\hline Min. & & & & & 490 & & & & & & & & & \\
\hline
\end{tabular}

b) rein, oder vorherrschend, untere Grenze

\begin{tabular}{|c|c|c|c|c|c|c|c|c|c|c|c|c|c|c|}
\hline sinumus & & 8897 & 3701 & $1261 \mathrm{x}$ & 1.203i & 5512 & $1 \mathrm{SH} H)$ & $327 !+$ & 3031 & $3 x ! 11 ;$ & $18+3$ & 6028 & $390 \overline{10 i}$ & 52303 \\
\hline Anzahl & 응 동 & 9 & 3 & 12 & 13 & 15 & 2 & 3 & 2 & 4 & 2 & 6 & 37 & 49 \\
\hline Mittel & It & 989 & $1: 2,0$ & 10int & 1172 & $110 x$ & $(H) O$ & 1048 & 1015 & $\pi .4$ & 932 & 1005 & 11172 & 1067 \\
\hline Min. & w & & & & & & 554 & & & & & & & \\
\hline
\end{tabular}

c) geschlossener Bestand, obere Grenze

\begin{tabular}{|c|c|c|c|c|c|c|c|c|c|c|c|c|c|c|}
\hline Smumnk & & $240 ; 8$ & 12855 & $36 \div+13$ & 5393i & $4173 i$ & iffivis 1 & 3037- & $\{30760$ & 2.579 & $37 \times 1 \pi$ & 40.516 & $310+6 !$ & 317342 \\
\hline Anzalil & & 10 & 8 & 34 & 32 & $2 f$ & if & 18 & 12 & 15 & 22 & 24 & 183 & 307 \\
\hline Mittel & & $15 \times 4$ & 1607 & 1538 & $\left.16 \bar{x}_{i}\right)$ & 1721 & 1072 & $16 \times 2$ & 1730 & $1,0,5$ & 1719 & 1688 & $169 \%$ & 1678 \\
\hline Max. & & & & & & & & & & & & 1858 & & \\
\hline
\end{tabular}

d) Raumbestand, obere Grenze (Waldgrenze)

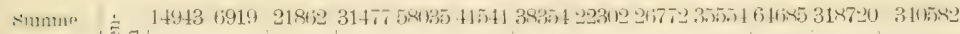

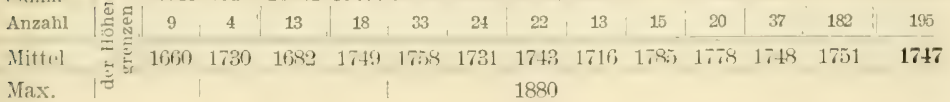




Berglehnen nach ihrer Exposition

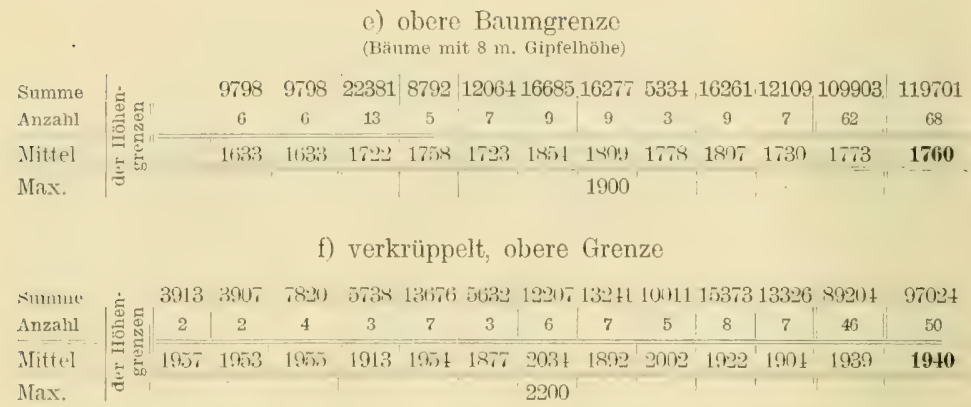

Abies pectimata DC. (A, alba Hill.)

a) vereinzelt, untere Grenze

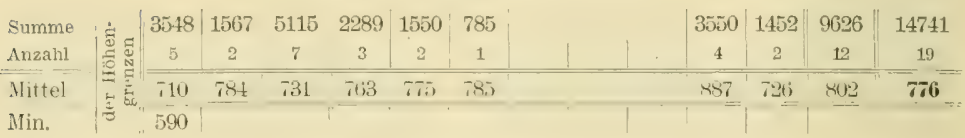

b) rein, oder vorherrschend, untere Grenze

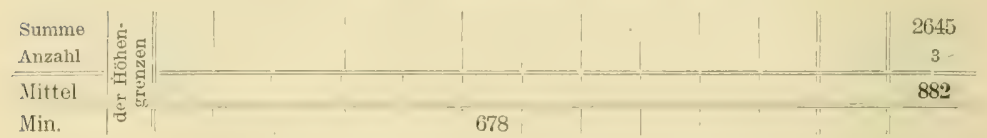

c) rein, oder vorherrschend, obere Grenze

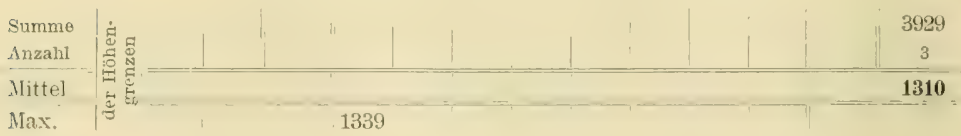

d) vereinzelt (Baumform), obere Grenze

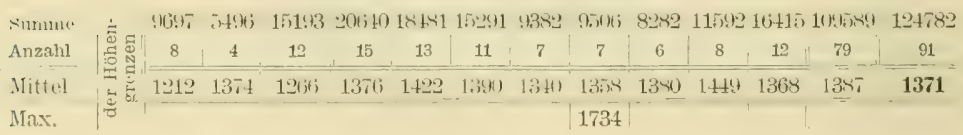




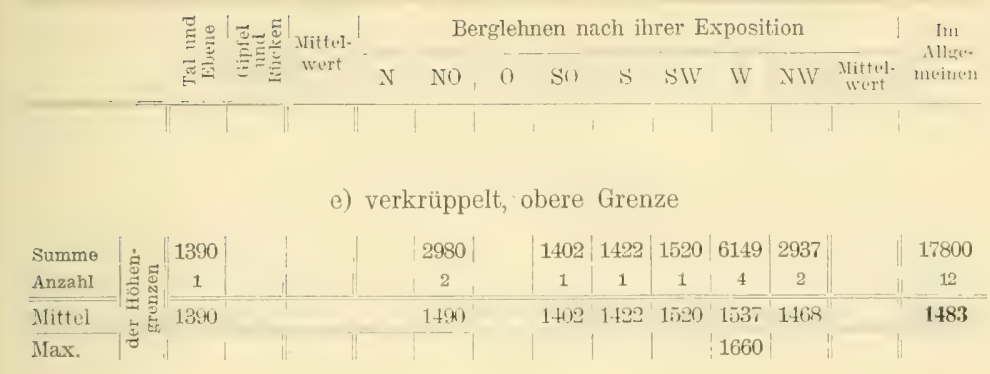

\section{Pimus Cembra $T_{\text {. }}$.}

a) untere Grenze

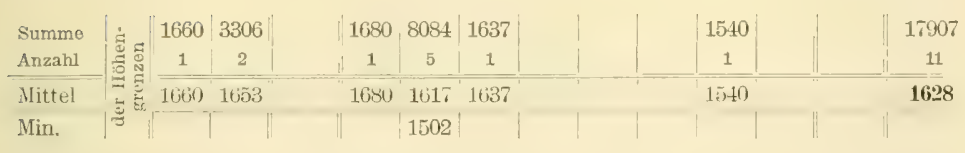

b) Baumform, obere Grenze

\begin{tabular}{|c|c|c|c|c|c|c|c|c|c|}
\hline Summe & 8999 & 9159 & 9191 & 7362 & 11357 & 1923 & 9229 & 14552 & 71772 \\
\hline Anzalil & 5 & 5 & 5 & 4 & ii & 1 & 5 & 8 & 34 \\
\hline Mitt.I & 1800 & 1832 & $1 . \times 38$ & 1810 & 1803 & 1923 & $18+f$ & 1819 & $18+0$ \\
\hline Max. & & & & & 1986 & & & & \\
\hline
\end{tabular}

c) verkrüppelt, obere Grenze

\begin{tabular}{|c|c|c|c|c|c|c|}
\hline Summe & 3734 & 1860 & 1899 & 1981 & 1971 & 11445 \\
\hline Anzahl & 2 & 1 & 1 & 1 & 1 & 6 \\
\hline Mittel & 1867 & $18(x)$ & 1899 & 1981 & 1971 & 1908 \\
\hline Max. & & i! & & 1981 & & \\
\hline
\end{tabular}

\section{Pinus montana Mill.}

a) sporadisch, untere Grenze

\begin{tabular}{l} 
Anzahl \\
\hline Mittel
\end{tabular}




-

b) massenhaft, untere Grenze

\begin{tabular}{l|c|c|c|c|c} 
Summe \\
Anzahl \\
\hline Mittrl
\end{tabular}

c) massenhaft, obere Grenzt

\begin{tabular}{l|c|c|c|c|c|c|c} 
Summe \\
Anzahl
\end{tabular}

d) sporadisch, obere Grenze

\begin{tabular}{|c|c|c|c|c|c|c|c|c|c|c|c|c|c|c|}
\hline Summe & & 3972 & 8521 & 19548 & 10606 & 4675 & $166 x y$ & $63 \overline{45}$ & $\operatorname{cins}$ & 2188 & 6470 & $.4+83$ & 56283 & 68870 \\
\hline Anzahl & 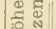 & 2 & 4 & 6 & 5 & 4 & 3 & 3 & 3 & 1 & 3 & 4 & 26 & 32 \\
\hline Mittel & 5 & 1946 & 2105 & 3099 & 2121 & 2169 & 2.288 & 2105 & 2196 & 2188 & 2157 & 2121 & 2165 & 2152 \\
\hline Iax. & & & & & & & $23(\pi)$ & & & & & & & \\
\hline
\end{tabular}

\section{Juniperus nana Willd.}

a) sporadisch, untere Grenze

\begin{tabular}{|c|c|c|c|c|c|c|c|c|c|c|}
\hline Summe & & 1390 & 3525 & 2670 & 3746 & 1410 & 1413 & 2946 & 5345 & 22445 \\
\hline Anzahl & & 1 & 3 & 2 & 3 & 1 & 1 & 3 & 5 & 19 \\
\hline Mittel & Fin & & & & & & & & & 1181 \\
\hline Min. & & & & & & & & 622 & & \\
\hline
\end{tabular}

b) massenhaft, untere Grenze

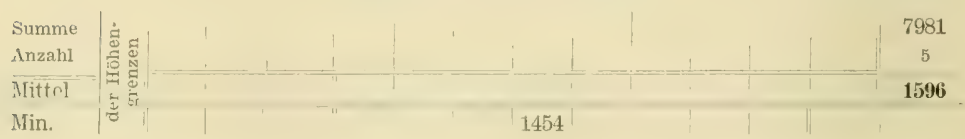

c) massenhaft, obere Grenze

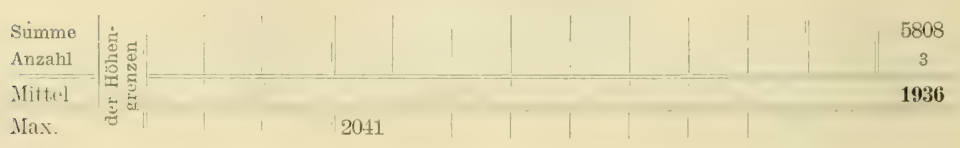




\begin{tabular}{|c|c|c|c|c|c|c|c|c|c|}
\hline \multirow{2}{*}{ 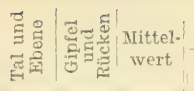 } & \multicolumn{8}{|c|}{ Berglelmen nach ihrer Exposition } & \multirow{2}{*}{$\begin{array}{l}\mathrm{Im} \\
\text { Allge- } \\
\text { meiner }\end{array}$} \\
\hline & $\mathrm{N}$ & NO & 0 & SO & S & SW! W & NW & $\begin{array}{l}\text { Mittel- } \\
\text { wert }\end{array}$ & \\
\hline
\end{tabular}

d) sporadisch, obere Grenze

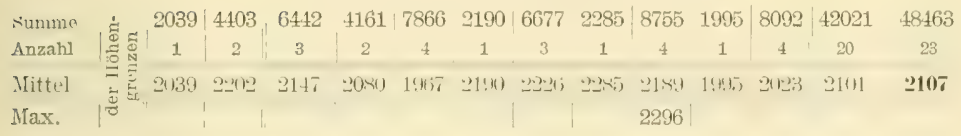

\section{Juniperus Sabina L.}

Min.

756

Max.

1060

\section{Fagus silvatica $\mathbf{L}_{\text {。 }}{ }^{*}$}

a) Bestandbildung im Allgemeinen, obere Grenze

\begin{tabular}{|c|c|c|c|c|c|c|c|c|c|c|c|c|c|c|}
\hline Sunitne & & 1 1i.., if & 26143 & 18139 & 213.2 & $: 11: 21$ & 211633 & 136 tis & $11 k+3$ & $\sin 0$ & $1329 \pi$ & $21+35$ & $1: 0011$ & 13845 \\
\hline Anzahl & 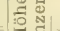 & 14 & 2 & 16 & 16 & 7 & 15 & 10 & 8 & 7 & 10 & 16 & 89 & 105 \\
\hline Mittel & है & 1125 & $13 \pm$ & 1152 & 1337 & $131 \%$ & 13.6 & 1367 & $131 i 1$ & 1311 & 1.330 & $13 i 2$ & $13+x$ & 1319 \\
\hline Max. & |こ & & & & & & & 1560 & & & & & & \\
\hline
\end{tabular}

$a_{1}$ ) obere Grenze der Bestandbildung, als Waldgrenze

\begin{tabular}{|c|c|c|c|c|c|c|c|c|}
\hline Summe & $\dot{\tilde{z}}$ & 1398 & 1400 & 1480 & 1398 & 1398 & 1398 & 8472 \\
\hline Anzahl & @륭 & 1 & 1 & 1 & 1 & 1 & 1 & 6 \\
\hline Mittel & $-\frac{5}{2}$ & $13 \pi \mathrm{s}$ & 1100 & $1+40$ & 1iveth & $130 \mathrm{x}$ & $13 \% k$ & $1+12$ \\
\hline Max. & 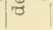 & & & 1480 & & & & \\
\hline
\end{tabular}

$a_{2}$ ) obere Grenze der Bestandbildung, darüber Fichte

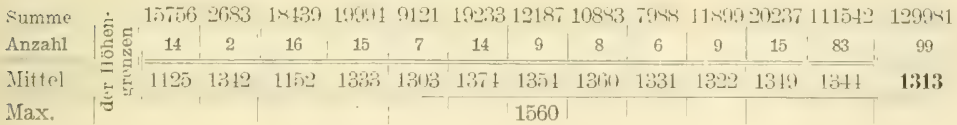

b) sporadisches Vorkommen im Fichtenbestand, obere Grenze

\begin{tabular}{|c|c|c|c|c|c|c|c|c|c|c|c|c|c|c|}
\hline Summe & & 20059 & 4217 & 21276 & 26591 & $1,1,93$ & 33749 & 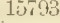 & 11401 & 18310 & 19390 & 265 . & 1718.2 & 190148 \\
\hline Anzahl & $\cong 5$ & 17 & 3 & 20 & 19 & 14 & 24 & 11 & 8 & 13 & 14 & 19 & 122 & 142 \\
\hline Mittel & $\therefore$ है & 1180 & $140 t^{2}$ & $1: 11$ & $135 !$ & $1+14$ & 1406 & 1436 & 1425 & $1+119$ & 1:inis & $1+13$ & 140.1 & 1381 \\
\hline Max. & $\pi$ & & & & & & & 1600 & & & & & & \\
\hline
\end{tabular}

* Erreicht ihre untere Grenze im Siebenbürgischen Becken. 


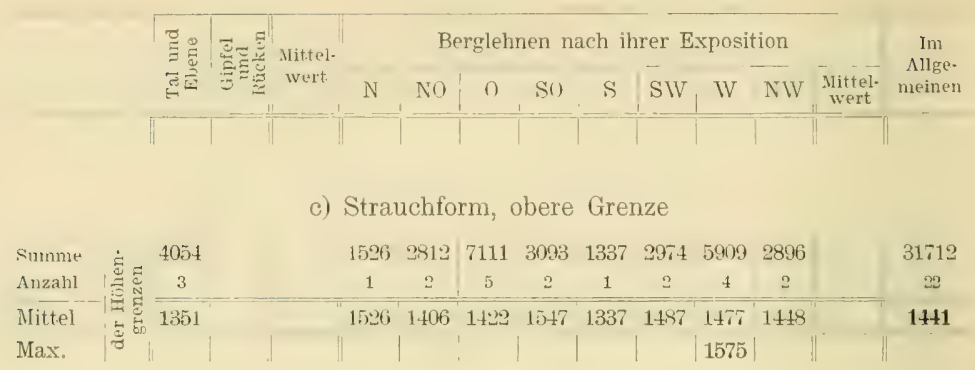

\section{Quercus sessiliflora Salisb.}

a) rein, oder vorherrschend, obere Grenze

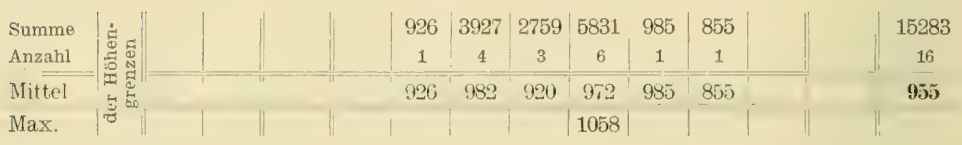

b) vereinzelt (Baumform), obere Grenze

\begin{tabular}{|c|c|c|c|c|c|c|c|c|c|c|}
\hline Summe & & 684 & 544 & 918 & 7824 & 5200 & 3283 & 2377 & 1965 & 22855 \\
\hline Anzahl & 몽 & 1 & 1 & 1 & 8 & 5 & 3 & 2 & 2 & 23 \\
\hline Mittel & 5 & 684 & $54 t$ & 948 & 978 & $10+1)$ & 1094 & 1189 & $9 \times 3$ & 992 \\
\hline Max. & 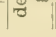 & & & & & & & $\mid 1217$ & & \\
\hline
\end{tabular}

Quercus pedunculata Ehrh. (Qu. Robur L.)

\begin{tabular}{|c|c|c|c|c|c|c|c|c|c|}
\hline $\begin{array}{l}\text { Summe } \\
\text { Anzahl }\end{array}$ & 运 & $\begin{array}{c}882 \\
2\end{array}$ & $\begin{array}{c}680 \\
1\end{array}$ & $\begin{array}{c}680 \\
1\end{array}$ & $\begin{array}{c}600 \\
1\end{array}$ & $\begin{array}{c}2550 \\
4\end{array}$ & $\begin{array}{c}600 \\
1\end{array}$ & $\begin{array}{c}3199 \\
5\end{array}$ & $\begin{array}{c}9191 \\
15\end{array}$ \\
\hline Mittel & 금 & 411 & 680 & 680 & 600 & 638 & 600 & 640 & 613 \\
\hline Max. & $\pi$ & & & & & & & 686 & \\
\hline
\end{tabular}

\section{Carpinus Betulus I.}

a) Baumform, obere Grenze

\begin{tabular}{|c|c|c|c|c|c|c|c|c|c|c|c|c|}
\hline Siumme & 4814 & 4814 & +130 & 900 & $618^{2}$ & 4716 & 5853 & 1903 & 4470 & 2716 & 30909 & 35723 \\
\hline Anzah & fi & 6i & 5 & 1 & 7 & 5 & 6 & 2 & 5 & 3 & 34 & 40 \\
\hline ittel & 802 & 802 & $8: 4$ & 900 & 883 & 943 & 975 & $97 \pi$ & 894 & 105 & 909 & 893 \\
\hline Max. & & & & & & & 1188 & & & & & \\
\hline
\end{tabular}




욜

b) Strauchform, obere Grenze

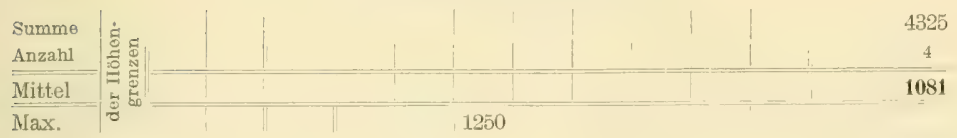

\section{Corylus Arellana L.}

obere Grenze

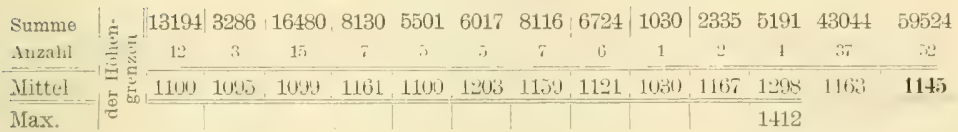

\section{Betula verrucosa Elirh.}

a) Baumform, obere Grenze

\begin{tabular}{|c|c|c|c|c|c|c|c|c|c|c|c|}
\hline Summe & $\dot{\approx}$ & 1297 & 3973 & 1623 & 8180 & 8789 & 8998 & 5750 & 1492 & | 2935 & 43037 \\
\hline Anzahl & $\stackrel{0}{\approx} \approx$ & 1 & 3 & 1 & 6 & 7 & 7 & 4 & 1 & 2 & 32 \\
\hline Mittel & $=$ & 1257 & 1321 & $10 i \div 3$ & $13(i)$ & $12 \pi x i$ & $12+i 5$ & $1+34$ & 1492 & $1+t i s$ & \multirow[t]{2}{*}{$13+5$} \\
\hline Max. & | & & & & & & & 1633 & & & \\
\hline
\end{tabular}

b) Strauchform, obere Grenze

\begin{tabular}{|c|c|c|c|c|c|c|c|c|c|c|}
\hline Summe & & $63 \pm 6$ & 8857 & 5559 & 5020 & 1422 & 1.414 & 4994 & 8822 & 12434 \\
\hline Anzahl & $\frac{B}{8} \bar{N}$ & 4 & 6 & 4 & 3 & 1 & 1 & 3 & 6 & 28 \\
\hline Mittel & 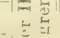 & 1.28 & 1.7 .6 & 1390 & 167.3 & $1+20$ & 1.11 & $16(6))$. & 1170 & 1516 \\
\hline Max. & 10 & & & & 1825 & & & & & \\
\hline
\end{tabular}

\section{Alnus glutinosa Giatn.}

obere Grenze

\begin{tabular}{|c|c|c|c|c|c|c|c|c|c|c|c|c|}
\hline Summe & & 13378 & 820 & 910 & 1010 & $\mid 1868$ & 968 & 2145 & 1659 & 1525 & 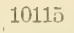 & 24313 \\
\hline Anzahl & $\cong 8:$ & 18 & 1 & 1 & 1 & 2 & 1 & 2 & 2 & 2 & 11 & 30 \\
\hline Mittel & 出焉 & 743 & 830 & 910 & 1040 & 934 & 968 & 1072 & 831 & $7(1) 2$ & (19) & 810 \\
\hline fax. & & & & & & & & 1250 & & & & \\
\hline
\end{tabular}




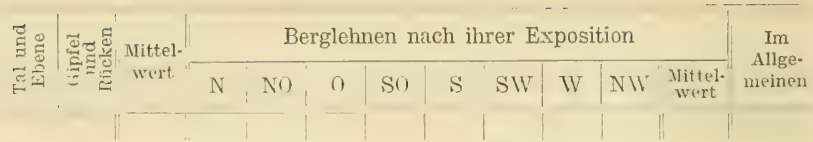

\section{Alnus incana Willd.}

obere Grenze

\begin{tabular}{|c|c|c|c|c|c|c|c|c|c|c|c|c|}
\hline Summe & & 22533 & 22533 & 1630 & 593 & 2655 & 860 & 1635 & 1635 & 3100 & 12108 & 34941 \\
\hline Anzah! & $\frac{9}{10} \frac{a}{N}$ & 25 & 25 & 2 & 1 & 3 & 1 & 2 & 2 & 4 & 15 & 40 \\
\hline Mittel & $\mid \begin{array}{ll}1 & 5 \\
5 & 5 \\
5 & 5\end{array}$ & 901 & 901 & 815 & 593 & 885 & 840 & 817 & 817 & 850 & 827 & 874 \\
\hline Max. & & 1281 & & & & & & & & & & \\
\hline
\end{tabular}

Alnus viridis 00 .

a) untere Grenze

\begin{tabular}{l|c|c|c|c|c|c|c|c|c|} 
Summe \\
Anzahl \\
\hline Mittel
\end{tabular}

b) obere Grenze

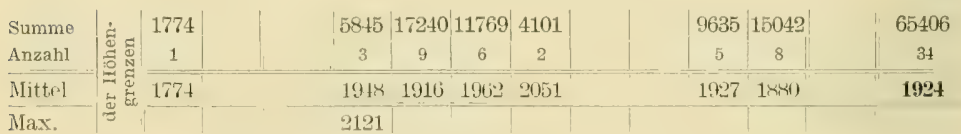

\section{Ulmus montama With. (U. seabra Mill.)}

a) Iuntere Grenze

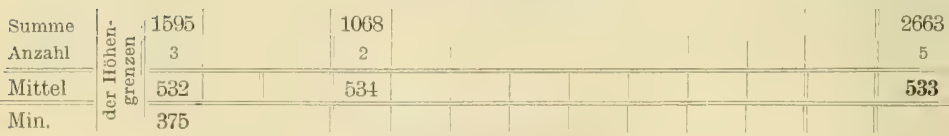

b) Baumform, obere Grenze

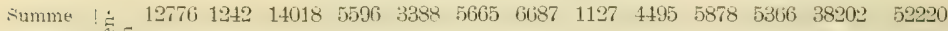
\begin{tabular}{l|ccccccccccccc} 
Anzahl \\
Mittel \\
Max.
\end{tabular} 


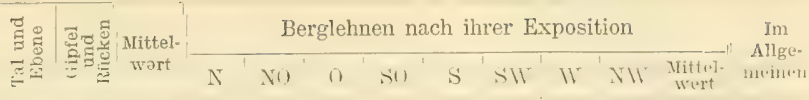

c) Strauchtorm, obere Grenze

\begin{tabular}{l|c|c|c|c} 
Summe \\
Anzahi \\
\hline Mittel
\end{tabular}

\section{Acer platanoides L.}

vereinzelt (Baumform), obere Grenze

\begin{tabular}{|c|c|c|c|c|c|c|c|c|c|c|}
\hline Summe & & 913 & 1196 & 1200 & 963 & 3193 & | 4268 | & | 2308 | & 1676 & 15747 \\
\hline Anzahl & 迎 & 1 & 1 & 1 & 1 & 3 & 4 & 2 & 2 & 15 \\
\hline Mittel & 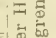 & 913 & 1196 & 1200 & 96.3 & 1064 & 1067 & 1154 & 838 & 1050 \\
\hline Max. & & & & & & & & 1210 & & \\
\hline
\end{tabular}

\section{Acer Pseudoplatanus L.}

a) vereinzelt (Baumform), obere Grenze

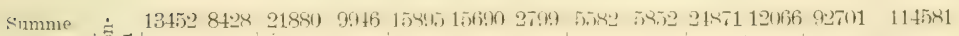

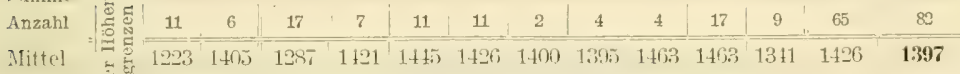
Max. 1652

b) verkrüppelt, obere Grenze

\begin{tabular}{|c|c|c|c|c|c|c|c|c|c|}
\hline Summe & & 1413 & 2819 & 5011 & 1780 & 1455 & 3166 & 4652 & 20296 \\
\hline Anzahl & 1 & 1 & 2 & 3 & 1 & 1 & 2 & 3 & 13 \\
\hline Mittel & 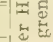 & & & & & & & & 1561 \\
\hline Max. & $1 \%$ & & & 1828 & & & & & \\
\hline
\end{tabular}

\section{Fraxinus excelsior L.}

a) vereinzelt (Baumform), obere Grenze

\begin{tabular}{|c|c|c|c|c|c|c|c|c|c|c|c|c|c|c|}
\hline Summo & & 7532 & 2180 & 9712 & 3952 & 3082 & 6790 & 5438 & 900 & 1220 & 1837 & 3757 & 26976 & 3668 \\
\hline Anzahl & 胥 & 8 & 2 & 10 & 4 & 3 & 6 & 5 & 1 & 1 & 2 & 4 & 26 & 36 \\
\hline Mittel & 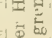 & 412 & $10: 31$ & 9.1 & $6 \times 8$ & $11+27$ & $11: 32$ & 10kis? & \{k11 & $1 \geq 20$ & .919 & 989 & 1038 & 1019 \\
\hline Max. & $\mathscr{Z}$ & & & & & & 1315 & & & & & & & \\
\hline
\end{tabular}




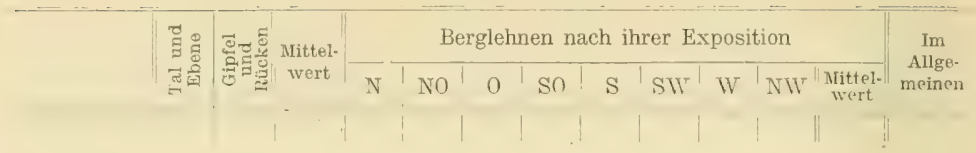

b) verkrüppelt, obere Grenze

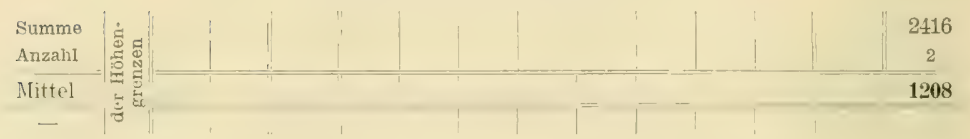

Sorbus ancuparia $\mathbf{L}$.

a) Baumform, obere Grenze

\begin{tabular}{|c|c|c|c|c|c|c|c|c|c|c|c|c|c|}
\hline Summe & & 17517 & 7517 & 9071 & 7355 & 7185 & 1370 & 2875 & 1528 & 3090 & 7532 & 40006 & 47523 \\
\hline Anzahl & $\div 5$ & 6 & 6 & 6 & 5 & 5 & 1 & 2 & 1 & 2 & 5 & 27 & 33 \\
\hline Mlittel & $=\check{\xi}_{0}$ & $12 \pi 3$ & $19 \pi$ & 1512 & $14 \pi j$ & $1+: 3$ & 1370 & $14: 18$ & $15: 28$ & 1545 & $1 ; 0 \mathrm{tj}$ & $1+\times 2$ & 1440 \\
\hline Max. & & & & & 1646 & & & & & & & & \\
\hline
\end{tabular}

b) Strauchform, obere Grenze

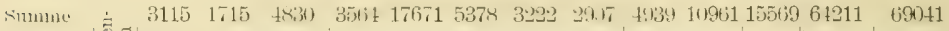

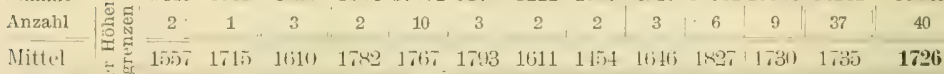
Max. $\left|{ }^{2}\right|+1987$

\section{Sambucus racemosa $\mathbf{L}$.}

a) untere Grenze

\begin{tabular}{|c|c|c|c|c|c|c|c|}
\hline Summe & & 765 & 775 & 528 & 1037 & 1189 & 765 \\
\hline Anzahl & 足 & 1 & 1 & 1 & 1 & 1 & 1 \\
\hline Mittel & 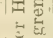 & $76 \tilde{0}$ & 7.5 & 538 & 1037 & 1189 & 流 \\
\hline Min. & $\bar{z}$ & & & 528 & & & \\
\hline
\end{tabular}

b) obere Grenze

\begin{tabular}{|c|c|c|c|c|c|c|c|c|c|c|c|}
\hline Summe & & 1106 & 7564 & 6008 & 8132 & 4970 & 1460 & 3082 & 4880 & 9160 & 46362 \\
\hline Anzahl & 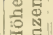 & 1 & 5 & 4 & 5 & 3 & 1 & 2 & 3 & 6 & 30 \\
\hline Mittel & $\Rightarrow$ & 1106 & 1513 & 1503 & $162 t ;$ & $16 i \pi$ & 1400 & $15+1$ & 1627 & 1527 & 1545 \\
\hline Max. & & & & & & & & & 1720 & & \\
\hline
\end{tabular}




\section{Siidungarisches Gebirgsland.}

Godján 2229 in., Gugu 2294 m., Szárko 2196 m., Vrf. Petri 2195 m., Szemenik 1419 m., Pojána Ruszka (Págyes) $1380 \mathrm{~m}$.

Berglehnen nach ihrer Exposition

\section{Picea excelsa Lk.}

a) vereinzelt, untere Grenze

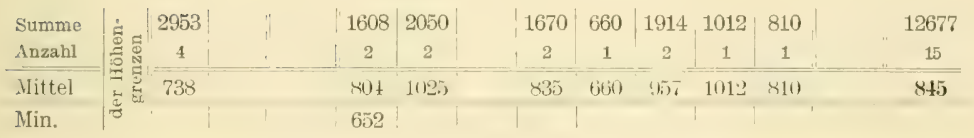

b) rein, oder vorherrschend, untere Grenze

\begin{tabular}{|c|c|c|c|c|c|c|c|}
\hline Summe & & 938 & 975 & 975 & 1170 & 2170 & 6228 \\
\hline Anzahl & 总 9 & 1 & 1 & 1 & 1 & 2 & 6 \\
\hline Mittel & $=$ & 938 & 975 & 975 & 1170 & 10,85 & 1038 \\
\hline Min. & & 988 & & & & & \\
\hline
\end{tabular}

c) geschlossener Bestand, obere Grenze

\begin{tabular}{|c|c|c|c|c|c|c|c|c|c|c|c|c|c|c|}
\hline Summe & & 4313 & 4779 & 9092 & 7633 & 3141 & 11075 & 4737 & 4647 & 2956 & 3066 & 3070 & 40325 & 49417 \\
\hline Anzahl & 造 & 3 & 3 & 6 & 5 & 2 & 7 & 3 & 3 & 2 & 2 & 2 & 26 & 30 \\
\hline Mittel & 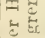 & 1434 & 1593 & 1515 & 1597 & 1571 & 1583 & 1579 & 1549 & 1478 & 1533 & 1035 & $|5 \tilde{n}|$ & 1544 \\
\hline Max. & & & & & 1685 & & & & & & & & & \\
\hline
\end{tabular}

d) Raumbestand, obere Grenze (Waldgrenze)

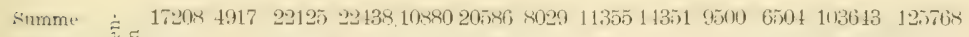

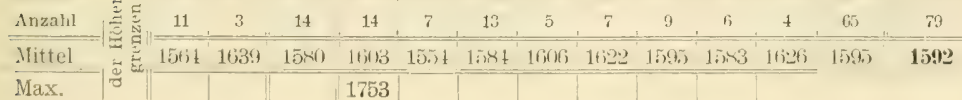




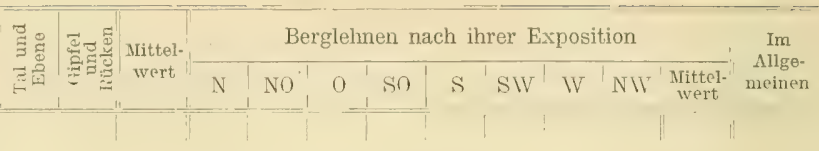

e) obere Baumgrenze

(Bäume mit $8 \mathrm{~m}$. Gipfelhöhe.)

\begin{tabular}{|c|c|c|c|c|c|c|c|c|c|c|c|c|c|c|}
\hline sumsure & \multirow{4}{*}{ 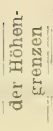 } & 20166 & 1550 & 22016 & 19818 & 8014 & 19357 & $(5,506$ & 8000 & 11125 & 15802 & 4645 & 935567 & 115583 \\
\hline Anzahl & & 13 & 1 & 14 & 12 & 5 & 12 & 4 & 5 & 7 & 10 & 3 & 58 & 72 \\
\hline Mittel & & 1574 & 1550 & 15.3 & If 52 & 1603 & 1613 & $162 \%$ & 1600 & 1632 & 1580 & 1518 & 1613 & 1605 \\
\hline Max. & & & & & & & 1828 & & & & & & & \\
\hline Summe & & 3309 & & & & 5681 & & & 3547 & & 3620 & & & 16160 \\
\hline Anzahl & 乐 & 2 & & & & 3 & & & 2 & & 2 & & & 9 \\
\hline Mittel & 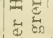 & 16,5 & & & & $18: 98$ & & & 177 & & 1810 & & & 1796 \\
\hline Max. & 101 & & & & . & 2100 & & & & & & & & \\
\hline
\end{tabular}

Abies peetiuata I)C. (A. alba Hill.)

a) vereinzelt, untere Grenze

\begin{tabular}{|c|c|c|c|c|c|c|c|c|c|c|c|c|c|c|}
\hline $\operatorname{Sum} \theta$ & & 9112 & $146 \pm$ & 2376 & 1997 & 5168 & 1567 & 1231 & 3035 & $2+88$ &,$\overline{3} 318$ & 2450 & 26256 & 28632 \\
\hline Anzahl & & 2 & 2 & 4 & 9 & 7 & 2 & 2 & 4 & 4 & 8 & 3 & 39 & 43 \\
\hline Mitted & $\Rightarrow 5$ & 456 & $73: 2$ & 514 & 555 & 738 & 783 & (i16 & 709 & 622 & 665 & 817 & 673 & 666 \\
\hline Min. & 5 & 327 & & & & & & & & & & & & \\
\hline
\end{tabular}

b) vereinzelt (Baumform), obere Grenze

\begin{tabular}{|c|c|c|c|c|c|c|c|c|c|c|c|c|c|c|}
\hline Summe & \multirow{4}{*}{ 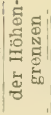 } & 5768 & 3872 & 9640 & 7118 & 2550 & 1488 & 8364 & 5570 & 2921 & 6892 & 2760 & 37663 & 47303 \\
\hline Anzahl & & 5 & 3 & 8 & 5 & 2 & 1 & 6 & 4 & 2 & 5 & 2 & 27 & 35 \\
\hline Mittel & & 1154 & $1: 91$ & 1205 & $142 t$ & 1.275 & 1488 & 1394 & 1393 & $1+61$ & 1378 & 1380 & 1395 & \multirow[t]{2}{*}{$13 \tilde{2}$} \\
\hline Max. & & & & & 1545 & & & & & & & & & \\
\hline
\end{tabular}

c) verkrüppelt, obere Grenze

\begin{tabular}{|c|c|c|c|c|c|c|}
\hline Summe & 2328 & 4365 & 2863 & 1583 & 1483 & 12622 \\
\hline Anzahl & 2 & 3 & 2 & 1 & 1 & 9 \\
\hline Mittel & $\Rightarrow$ है $116 t$ & 1455 & $1+32$ & 1583 & 1483 & 1402 \\
\hline Max. & $\mid 0$ & & & 1583 & & \\
\hline
\end{tabular}

\section{Pimus nigra Aru.}

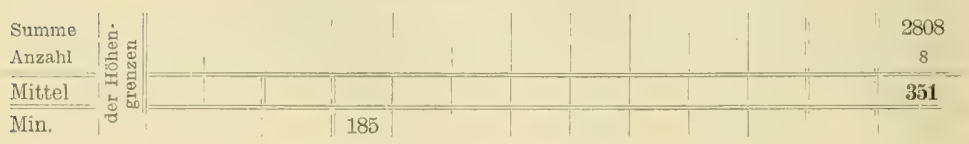




\begin{tabular}{|c|c|c|c|c|c|c|c|c|c|c|c|c|}
\hline \multirow{2}{*}{ 苛 : } & \multirow{2}{*}{ Ðేశ } & \multirow{2}{*}{$\begin{array}{l}\text { Nittel- } \\
\text { wert }\end{array}$} & \multicolumn{9}{|c|}{ Berglehnen nach ihrer Exposition } & \multirow{2}{*}{$\begin{array}{c}\text { Im } \\
\text { Allge- } \\
\text { meinen }\end{array}$} \\
\hline & & & $\mathrm{N}$ & NO & 0 & so & S & SW & W & NW & & \\
\hline
\end{tabular}

b) Baumform, obere Grenze

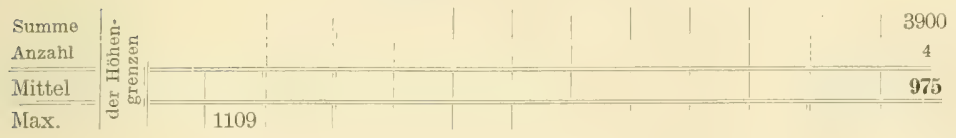

c) verkrüppelt, obere Grenze

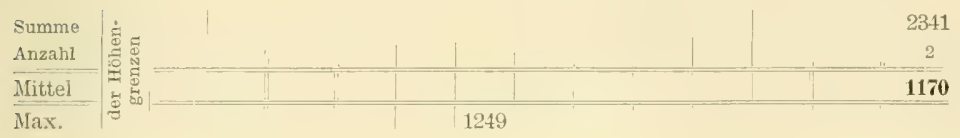

\section{Pimus Cembra I.}

a) untere Grenze

Min.

1628

b) obere Grenze

\begin{tabular}{l|c|c|c|c|c|c|c} 
Summe & Anzahl \\
\hline Mittel & Max. & 1851 & 3735 & 3670 & 1810 & 11066 \\
0 & 0 & 1 & 2 & 2 & 1 & 1 \\
\hline
\end{tabular}

\section{Pinus montana Mill.}

a) untere Grenze

\begin{tabular}{|c|c|c|c|c|c|c|c|c|c|}
\hline Summe & \& & 3126 & 3321 & 1628 & 1614 & $|1810|$ & 1291 & 1625 & 14415 \\
\hline Anzahl & $\left.\frac{0}{3}\right]^{E}$ & 2 & 2 & 1 & 1 & 1 & 1 & 1 & 9 \\
\hline Mittel & 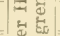 & & & & & & & & $160^{2}$ \\
\hline Min. & & & & & & & 1291 & & \\
\hline
\end{tabular}

b) obere Grenze

(keine) 


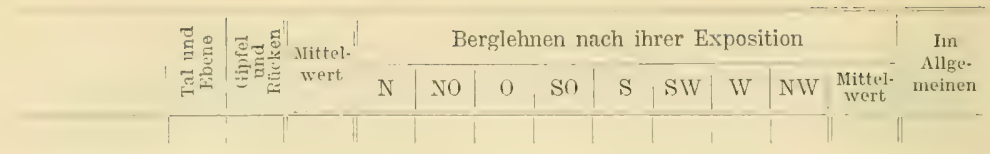

Taxus baceata $\mathbf{T}$.

a) untere Grenze

\begin{tabular}{l|c|c|c|c|c|c}
$\begin{array}{l}\text { Summe } \\
\text { Anzahl }\end{array}$ \\
$\begin{array}{l}\text { Mittel } \\
\text { Min. }\end{array}$
\end{tabular}

b) obere Grenze

\begin{tabular}{|c|c|c|c|c|c|}
\hline $\begin{array}{l}\text { Summe } \\
\text { Anzahl }\end{array}$ & $\begin{array}{c}1197 \\
1\end{array}$ & $\begin{array}{c}855 \\
1\end{array}$ & $\begin{array}{c}1120 \\
1\end{array}$ & $\begin{array}{c}936 \\
1\end{array}$ & $\begin{array}{c}4108 \\
4\end{array}$ \\
\hline Mittel & 1197 & 855 & $11: 0$ & 936 & 1027 \\
\hline Max. & 1197 & & & & \\
\hline
\end{tabular}

\section{Fagus silvatica $\mathbf{I}$.}

a) vereinzelt, untere Grenze

\begin{tabular}{|c|c|c|c|c|c|c|}
\hline Summe & 749 & 150 & 296 & 95 & 530 & 1820 \\
\hline Anzahl & 送 & 1 & 1 & 1 & 1 & 10 \\
\hline Mittel & $\begin{array}{lll}7 & 125 \\
4 & 5 & 1\end{array}$ & 150 & 296 & 95 & 530 & 182 \\
\hline Min. & 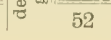 & & & & & \\
\hline
\end{tabular}

b) rein, oder vorherrschend, untere Grenze

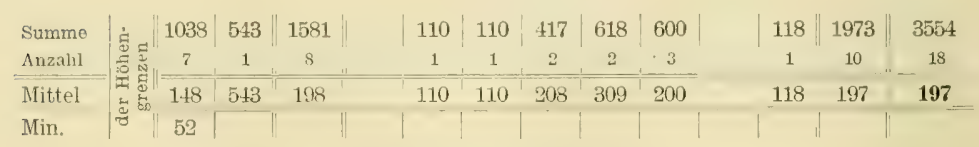

c) obere Grenze der Bestandbildung als Waldgrenze

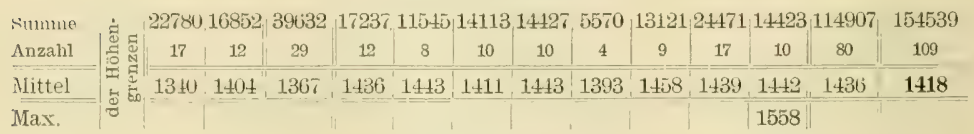

d) obere Grenze der Bestandbildung, darüber Fichte

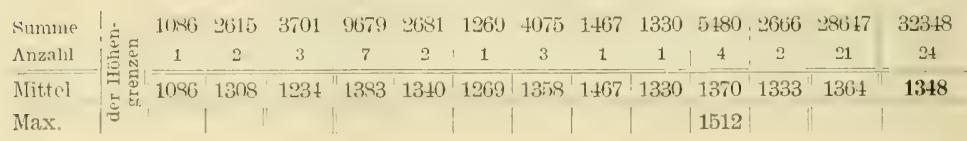




\begin{tabular}{|c|c|c|c|c|c|c|c|c|c|c|c|c|}
\hline \multirow{2}{*}{ 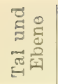 } & \multirow{2}{*}{ 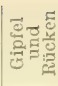 } & \multirow{2}{*}{$\begin{array}{l}\text { Mittel. } \\
\text { wert }\end{array}$} & \multicolumn{9}{|c|}{ Berglehnen nach ihrer Exposition } & \multirow{2}{*}{$\begin{array}{c}\text { Im } \\
\text { Allge- } \\
\text { meiner }\end{array}$} \\
\hline & & & $\mathrm{N}$ & NO & 0 & So & S & SW & WV & NWV & $\begin{array}{l}\text { Mittel- } \\
\text { wert }\end{array}$ & \\
\hline
\end{tabular}

e) sporadisches Vorkommen im Fichtenbestand, obere Grenze

\begin{tabular}{|c|c|c|c|c|c|c|c|c|c|c|c|c|}
\hline Summe & 6010 & 6010 & 7163 & 2967 & 10086 & 5846 & 4238 & 2760 & 11332 & 8526 & 52918 & 5892 \\
\hline AnzahI & 5 & 5 & 5 & 2 & 7 & 4 & 3 & 2 & 8 & 6 & 37 & 42 \\
\hline $\begin{array}{l}\text { Mittel } \\
\text { Max. }\end{array}$ & $1: 12$ & 1212 & 1133 & 1 tst & $\begin{array}{l}1 \mathrm{H1} \\
1560\end{array}$ & $14 t i 2$ & $1+1: 3$ & $1: 30$ & 1117 & 1431 & 11311 & \\
\hline
\end{tabular}

f) Strauchform, obere Grenze

\begin{tabular}{|c|c|c|c|c|c|c|c|c|c|c|c|c|c|}
\hline Summo & & 6910 & 6910 & 4571 & 1419 & 4178 & 4390 & 4353 & 1450 & 4382 & 5895 & 30638 & 37548 \\
\hline Anzahl & 怘 & 5 & 5 & 3 & 1 & 3 & 3 & 3 & 1 & 3 & 4 & 21 & 26 \\
\hline Alittel & $=$ & $13 \times 2$ & 1282 & list & $1+19$ & 139.3 & $1+1 ; 3$ & $14 \tilde{3} 1$ & $(4, \pi)$ & $1 .+61$ & $1+7.4$ & $14: 99$ & 1444 \\
\hline Max. & & & & & & & & & & & 1562 & & \\
\hline
\end{tabular}

\section{Quereus sessiliflora Salisb.}

a) rein, oder vorherrschend, obere Grenze

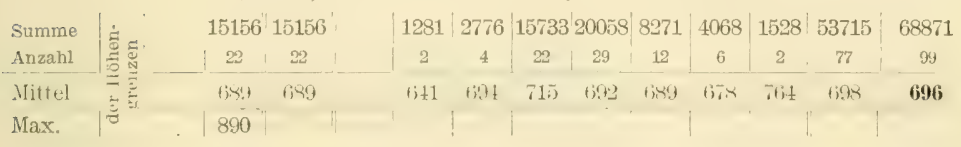

b) vereinzelt (Baumform), obere Grenze

\begin{tabular}{|c|c|c|c|c|c|c|c|c|c|c|c|c|c|c|}
\hline Summe & & 682 & 16633 & 17315 & 720 & 637 & 6988 & 11288 & 16060 & 9987 & 10263 & 1312 & $5725 \overline{5}$ & 74570 \\
\hline Anzahl & 胥 & 1 & 23 & 24 & 1 & 1 & 10 & 15 & 23 & 13 & 14 & 2 & 78 & 102 \\
\hline Mittel & 5 & fix:? & 723 & 721 & 720 & 15:3. & 1645 & 73 & $(: ; i)$ & itis & .33 & (int) & $73 t$ & \\
\hline Max. & & & & & & & & & & 992 & & & & \\
\hline
\end{tabular}

c) verkrüppelt, obere Grenze

\begin{tabular}{l|c|c|c|c|c|c}
$\begin{array}{l}\text { Summe } \\
\text { Anzahl }\end{array}$ \\
\hline Mittel \\
\hline Max.
\end{tabular}

(quercus peduneulata Ehrh. (Qu. Robur I.)

\begin{tabular}{l|l|c|c|c|c|c|c} 
Summe \\
Anzahl \\
$\begin{array}{l}\text { Mittel } \\
\text { Max. }\end{array}$ \\
\hline Max.
\end{tabular}




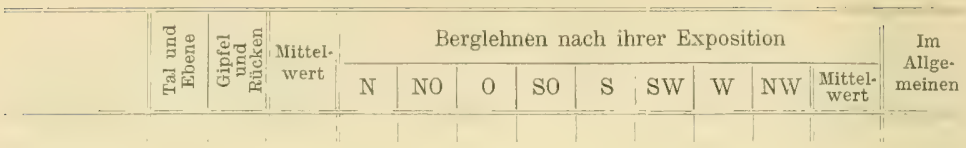

\section{Quereus conferta Kit.}

a) rein, oder vorhersschend, obere Grenze

\begin{tabular}{l|c|c|c|c|c|c|c|c|c} 
Summe & & 1545 & 359 & 697 & 952 & 1164 & 1350 & 344 & 6411 \\
Anzahl \\
\hline $\begin{array}{l}\text { Mittel } \\
\text { Max. }\end{array}$
\end{tabular}

b) vereinzelt, obere Grenze

\begin{tabular}{|c|c|c|c|c|c|c|c|c|c|}
\hline Summe & 3929 & 395 & 910 & 2244 & 4427 & 389 & 470 & 1194 & 13958 \\
\hline Anzahl & 8 & 1 & 2 & 4 & 8 & 1 & 1 & 2 & 27 \\
\hline Mittel & 491 & 305 & $45 \tilde{5}$ & 561 & 553 & 389 & 4.0 & 597 & 517 \\
\hline Max. & & & & 724 & & & & & \\
\hline
\end{tabular}

\section{Quereus Cerris $\mathbf{L}$.}

a) rein, oder vorherrschend, obere Grenze

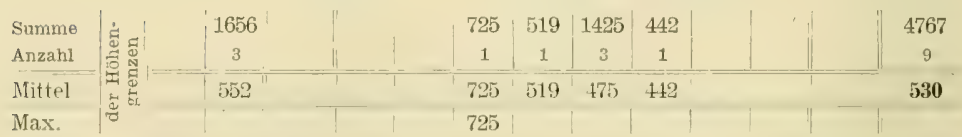

b) vereinzelt, obere Grenze

\begin{tabular}{|c|c|c|c|c|c|c|c|c|c|c|c|c|}
\hline $\begin{array}{l}\text { Summe } \\
\text { Anzahl }\end{array}$ & 동 & $\left|\begin{array}{c}3909 \\
6\end{array}\right|$ & $\begin{array}{c}3909 \\
6\end{array}$ & $\begin{array}{c}628 \\
1\end{array}$ & $\begin{array}{c}1772 \\
3\end{array}$ & $\begin{array}{c}1984 \\
3\end{array}$ & $\begin{array}{c}6619 \\
10\end{array}$ & $\begin{array}{c}2391 \\
4\end{array}$ & $\begin{array}{c}1415 \\
2\end{array}$ & $\begin{array}{c}5.18 \\
1\end{array}$ & $\begin{array}{c}15357 \\
24\end{array}$ & $\begin{array}{c}19266 \\
30\end{array}$ \\
\hline Iittel & ت5 & 652 & $(6,5)$ & 628 & 591 & 661 & 662 & 594 & 708 & 548 & 640 & 642 \\
\hline
\end{tabular}

\section{Juglans regia $\mathrm{L}$.}

obere Grenze

\begin{tabular}{|c|c|c|c|c|c|c|c|c|c|}
\hline Summe & & 1076 & 403 & 498 & 437 & 1018 & 630 & 1099 & 5161 \\
\hline Anzahl & ฐ & 3 & 1 & 1 & 1 & 3 & 1 & 2 & 12 \\
\hline Mittel & ज施 & 359 & 403 & 495 & 437 & 339 & 630 & 550 & 430 \\
\hline Max. & "ू. & & & & & & 630 & & \\
\hline
\end{tabular}




\begin{tabular}{|c|c|c|c|c|c|c|c|c|c|c|}
\hline \multirow{2}{*}{ 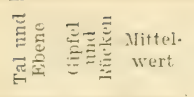 } & \multicolumn{9}{|c|}{ Berglehnen nach ihrer Exposition } & \multirow{2}{*}{$\begin{array}{l}\text { Im } \\
\text { Allge- } \\
\text { meinen }\end{array}$} \\
\hline & $\mathrm{N}$ & NO & 0 & SO & $\mathrm{S}$ & $S 11$ & IV & NWV & $\begin{array}{l}\text { Mittel- } \\
\text { wert }\end{array}$ & \\
\hline
\end{tabular}

\section{Carpinus Betulus L.}

a) Baumform, obere Grenze

\begin{tabular}{|c|c|c|c|c|c|c|c|c|c|c|c|c|c|c|}
\hline Summe & & 4322 & $7-21$ & 12043 & $16 i f f$ & 10) 10 & $2(1) 3$ & $53: 3 !+1$ & $8(1) 18$ & 1324 & $469+4$ & 3114 & 3121k & $12: 31 ; 1$ \\
\hline Anzahl & 동 & 6 & 9 & 15 & 2 & 1 & 3 & 7 & 10 & 5 & 6 & 4 & 38 & 53 \\
\hline Aittel & & 720 & 828 & 803 & 823 & $10+0$ & 6.94 & 761 & 802 & 805 & 782 & 779 & $79 \%$ & 797 \\
\hline Max. & 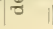 & & & & & & & & & & 1118 & & & \\
\hline
\end{tabular}

b) Strauchform, obere Grenze

\begin{tabular}{|c|c|c|c|c|c|c|c|}
\hline Summe & & 1003 & 1049 & 2064 & 1069 & 970 & 6155 \\
\hline Anzahl & $\cong$ & 1 & 1 & 2 & 1 & 1 & 6 \\
\hline Mitte] & $\bar{E}$ & 1003 & 1049 & 1032 & 1064 & 970 & 1026 \\
\hline- & | & & & & & & \\
\hline
\end{tabular}

\section{Carpinus dninensis Scop.}

\begin{tabular}{l|c|c|c|c|c|c|c|c|c|c} 
obere Grenze \\
Summe \\
Anzahl \\
\hline Mittel
\end{tabular}

\section{Corylus Arellaua L.}

\begin{tabular}{|c|c|c|c|c|c|c|c|c|c|}
\hline $\begin{array}{l}\text { Summe } \\
\text { Anzahl }\end{array}$ & \multirow{3}{*}{ 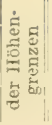 } & $\begin{array}{c}2145 \\
2\end{array}$ & $\begin{array}{c}1097 \\
1\end{array}$ & $\begin{array}{c}1140 \\
1\end{array}$ & $\begin{array}{c}1170 \\
1\end{array}$ & $\begin{array}{c}1328 \\
1\end{array}$ & $\mid \begin{array}{c}1125 \\
1\end{array}$ & $\begin{array}{c}3194 \\
3\end{array}$ & $\begin{array}{c}11199 \\
10\end{array}$ \\
\hline Nittel & & 1073 & 1097 & 11.40 & 1170 & 1328 & 1135 & 1065 & 1120 \\
\hline Max. & & & & & & 1328 & & & \\
\hline
\end{tabular}

\section{Corylus Colurua L.}

obere Grenze

\begin{tabular}{|c|c|c|c|c|c|c|c|}
\hline $\begin{array}{l}\text { Summe } \\
\text { Anzahl }\end{array}$ & $\left\{\begin{array}{l}\dot{a} \\
\frac{a}{2} \\
\frac{5}{5}\end{array}\right.$ & $\begin{array}{c}2068 \\
2\end{array}$ & $\begin{array}{c}1040 \\
1\end{array}$ & $\begin{array}{c}1740 \\
2\end{array}$ & $\begin{array}{c}980 \\
1 \\
\end{array}$ & $\begin{array}{c}1997 \\
2\end{array}$ & $\begin{array}{c}7825 \\
8 \\
\end{array}$ \\
\hline Ilittr. & $=5$ & 1031 & $10+0$ & 8.0 & 9811 & 999 & 978 \\
\hline Max. & $1 \tilde{\sigma}$ & 1069 & & & & & \\
\hline
\end{tabular}




\begin{tabular}{|c|c|c|c|c|c|c|c|c|c|c|c|c|}
\hline \multirow{2}{*}{ 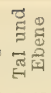 } & \multirow{2}{*}{ 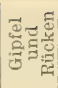 } & \multirow{2}{*}{$\begin{array}{c}\text { Mittel- } \\
\text { wert }\end{array}$} & \multicolumn{9}{|c|}{ Berglehnen nach ihrer Exposition } & \multirow{2}{*}{$\begin{array}{c}\text { Im } \\
\text { Allge- } \\
\text { meinen }\end{array}$} \\
\hline & & & $\mathrm{N}$ & No & 0 & so & $\mathrm{S}$ & SW & W & NW & $\mid \begin{array}{c}\text { Mittel- } \\
\text { wert }\end{array}$ & \\
\hline
\end{tabular}

Betula vermeosa Ehrh.

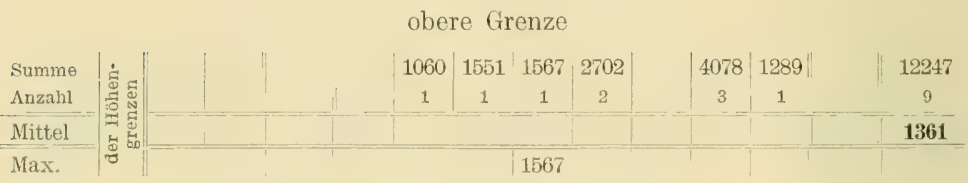

Aluus glutinosa Gärtu.

obere Grenze

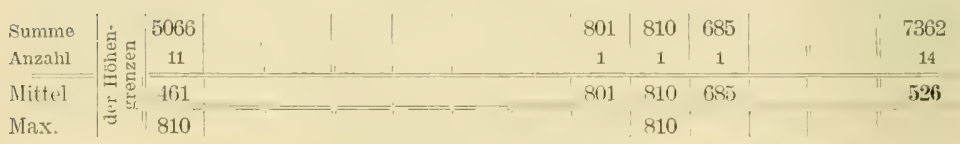

Aluus incana Willl.

obere Grenze

\begin{tabular}{l|c|c|c|c|c|c|c} 
Summe \\
Anzahl \\
\hline Mlittel
\end{tabular}

Alnus virilis DC.

a) untere Grenze

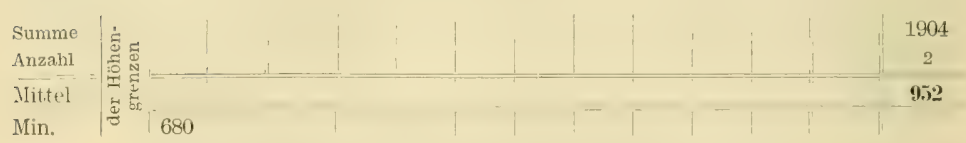

b) obere Grenze

\begin{tabular}{|c|c|c|c|c|}
\hline Summe & 4498 & 18543809 & 1562 & 11723 \\
\hline Anzahl & 3 & \begin{tabular}{l|l}
1 & 2
\end{tabular} & 1 & 7 \\
\hline Mittel & & & & 1675 \\
\hline Max. & & 1960 & & \\
\hline
\end{tabular}




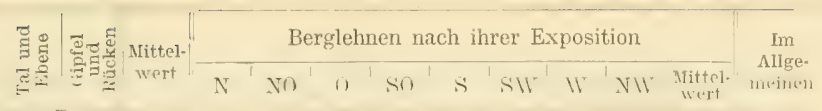

\section{Ulmus montana With. (U. scabra Mill.)}

a) intere Grenze

\begin{tabular}{|c|c|c|c|c|c|}
\hline Summe & $1272 !$ & 883 & 1368 & 345 & 3868 \\
\hline AnzahI & 3 & 2 & 3 & 1 & 9 \\
\hline Mittel & 424 & $14^{2}$ & 456 & 315 & 430 \\
\hline Min. & & & & 345 & \\
\hline
\end{tabular}

b) Baumform, obere Grenze

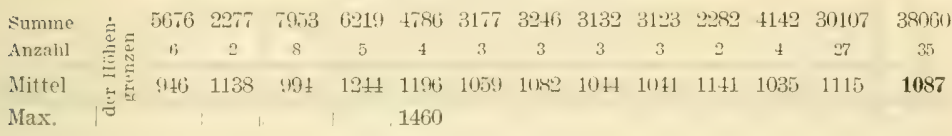

c) Strauchform, obere Grenze

\begin{tabular}{l|c|c|c|c} 
Summe \\
Anzahl \\
\hline Mittel
\end{tabular}

Tilia parrifolia Ehrh. (T. cordata Nill.)

\begin{tabular}{|c|c|c|c|c|c|c|c|}
\hline $\begin{array}{l}\text { Summe } \\
\text { Anzahl }\end{array}$ & $\begin{array}{c}1463 \\
2\end{array}$ & $\begin{array}{c}2522 \\
3\end{array}$ & $\begin{array}{c}1005 \\
1\end{array}$ & $\begin{array}{c}1112 \\
1\end{array}$ & $\begin{array}{c}3013 \\
3\end{array} \mid$ & $\begin{array}{c}879 \\
1\end{array}$ & 9994 \\
\hline Mittel & 732 & 841 & 100.5 & 1112 & 100.4 & 879 & 909 \\
\hline Max. & & & & & 1121 & & \\
\hline
\end{tabular}

\section{Tilia tomentosa Mönch.}

obere Grenze

\begin{tabular}{|c|c|c|c|c|c|c|c|c|c|c|c|c|c|c|}
\hline Summe & & 420 & 4703 & 5123 & 1507 & 1762 & 4293 & 1514 & 6421 & 1219 & 1343 & 916 & 18975 & 24098 \\
\hline Anzahl & & 1 & 7 & 8 & 2 & 2 & 6 & 2 & 9 & $\begin{array}{l}2 \\
\mid\end{array}$ & 2 & 1 & 26 & 34 \\
\hline Nittel & & 420 & 678 & t5:10 & 753 & 881 & 716 & $75 \pi$ & 713 & (ill) & 6.1 & 916 & 730 & 709 \\
\hline Max. & & & & & & & & & & & & 916 & & \\
\hline
\end{tabular}




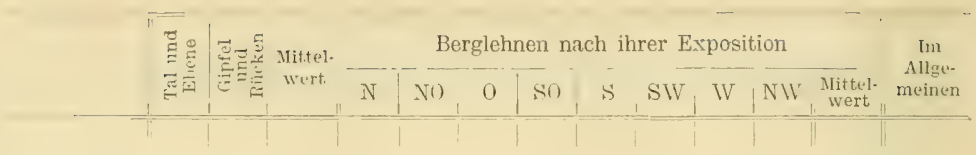

\section{Acer platanoides $L$.}

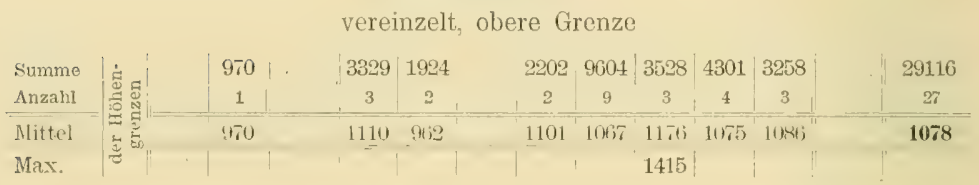

\section{Acer Psendoplatanus $\mathbf{L}$.}

vereinzelt, obere Grenze

\begin{tabular}{|c|c|c|c|c|c|c|c|c|c|c|c|c|c|c|}
\hline Summe & & 7145 & 7771 & 14916 & 12911 & 6116 & 8801 & 4039 & 3896 & 1225 & $\mid 12327$ & 2962 & $|52277|$ & 67193 \\
\hline Anzalil & एี & 6 & 6 & 12 & 9 & 4 & 6 & 3 & 3 & 1 & 9 & 2 & 37 & 49 \\
\hline Mittel & 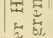 & 1791 & 1295 & 1213 & 1435 & 1509 & 1467 & $13+6$ & 1291 & $12 \%$ & 1370 & $1+r 1$ & 1413 & 1371 \\
\hline Max. & 12 & & & & & 1670 & & & & & & & & \\
\hline
\end{tabular}

\section{Acer campestre L.}

\begin{tabular}{|c|c|c|c|c|c|c|c|}
\hline Summe & 3722 & 1532 & 2053 & 400 & $4373|3059|$ & 1356 & 16495 \\
\hline Anzahl & 5 & 2 & 3 & 1 & $5 \quad 4$ & 2 & 22 \\
\hline Mitfel & 744 & Thif & likt & tou & 8,5 & $6 \pi$ & 750 \\
\hline Max. & & & & & 1104 & & \\
\hline
\end{tabular}

\section{Acer monspessulanum L.}

obere Grenze

Max.

240

\section{Fraxinus excelsior $I_{\text {. }}$}

vereinzelt, obere Grenze

\begin{tabular}{|c|c|c|c|c|c|c|c|c|c|c|c|c|c|c|}
\hline Sแmmนe & & 3185 & $5(1) 1)$ & 86,18 & 3967 & $\ddot{2}(x)$ & 3130 & 1235 & 3173 & 3345 & $2+90$ & 3711 & 2.451 & $314: 19$ \\
\hline Anzahl & 1ํํㅇ & 4 & 5 & 9 & 3 & 2 & 3 & 1 & 3 & 2 & 2 & 3 & 19 & 28 \\
\hline Mittel & & 797 & 1052 & 961 & 1302 & 1250 & $10 \$ 3$ & 1235 & 11 in & 1173 & 1245 & 1337 & 1203 & 1125 \\
\hline Max. & & & & & 1460 & & & & & & & & & \\
\hline
\end{tabular}




\begin{tabular}{|c|c|c|c|c|c|c|c|c|c|c|c|c|}
\hline \multirow{2}{*}{ 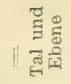 } & \multirow{2}{*}{ 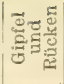 } & \multirow{2}{*}{$\begin{array}{c}\text { Mittel- } \\
\text { wert }\end{array}$} & \multicolumn{9}{|c|}{ Berglehnen nach ihrer Exposition' } & \multirow{2}{*}{$\begin{array}{c}\text { Im } \\
\text { Allge- } \\
\text { meinen }\end{array}$} \\
\hline & & & $N$ & NO & 0 & $\mathrm{SO}$ & $\mathrm{S}$ & SW & W & NW & $\begin{array}{c}\text { Mittel- } \\
\text { wert }\end{array}$ & \\
\hline
\end{tabular}

\section{Fraximus Ornus L.}

\begin{tabular}{|c|c|c|c|c|c|c|c|c|c|c|c|c|c|}
\hline $\begin{array}{l}\text { Summe } \\
\text { Anzahl }\end{array}$ & : & $\begin{array}{c}741 \\
1\end{array}$ & $\begin{array}{c}2240 \\
3\end{array}$ & $\begin{array}{c}2981 \\
4\end{array}$ & $\begin{array}{c}1481 \\
2\end{array}$ & $\begin{array}{c}639 \\
1\end{array}$ & $\begin{array}{c}2248 \\
3\end{array}$ & $\begin{array}{c}1317 \\
2\end{array}$ & $\begin{array}{c}3244 \\
4\end{array}$ & $\begin{array}{c}2943 \\
4\end{array}$ & $\begin{array}{c}5143 \\
7\end{array}$ & $\begin{array}{c}17015 \\
33\end{array}$ & $\begin{array}{c}19996 \\
27\end{array}$ \\
\hline Mittel & $\frac{\pi}{2}=$ & $7+1$ & 74 & $74 \tilde{i}$ & $7+1)$ & 633 & 719 & $6 i y_{1}$ & 811 & 736 & 735 & 740 & $7+1$ \\
\hline Max. & 1 & & & & & & & & 1103 & & & & \\
\hline
\end{tabular}

\section{Sorbus aucuparia $\mathbf{L}$.}

a) Baumform, obere Grenze

\begin{tabular}{|c|c|c|c|c|c|c|c|c|}
\hline Summe & & 1099 & 3304 & 1688 & | 3987 | & 1496 & 1390 & 12964 \\
\hline Anzahl & 용 & 1 & 2 & 1 & 3 & 1 & 1 & 9 \\
\hline Mittel & th & 1099 & 1652 & 1688 & 1329 & 1496 & 1390 & $1+40$ \\
\hline Max. & | & & 1753 & & & & & \\
\hline
\end{tabular}

b) Strauchform, obere Grenze

\begin{tabular}{|c|c|c|c|c|c|c|c|c|}
\hline Summe & & 4521 & $|1519|$ & 1628 & 1520 & $\mid 1388$ & 1580 & 12156 \\
\hline Anzahl & $\mid$ & 3 & 1 & 1 & 1 & 1 & 1 & 8 \\
\hline $\begin{array}{l}\text { Mittel } \\
\text { Max. }\end{array}$ & . & $\begin{array}{l}1507 \\
1851\end{array}$ & 1519 & 1628 & 1520 & $13 \times x$ & 1580 & 1520 \\
\hline
\end{tabular}

\section{Sorbus torminalis Crantz.}

\begin{tabular}{l||c|c|c|c|c|c|c|c|c|c|} 
Summe & $\mid 2524$ & 601 & 1633 & 1524 & 1457 & 2866 & 2706 & 608 & 919 & 14838 \\
Anzahl \\
\hline Mittel
\end{tabular}

\section{Cotinus Coggygria Scop.}

obere Grenze

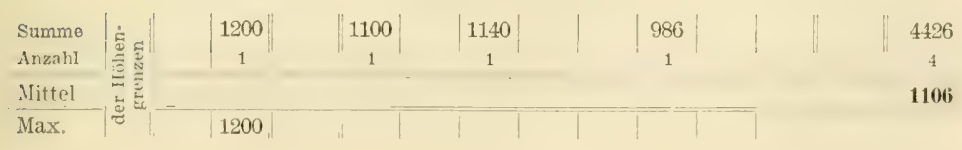




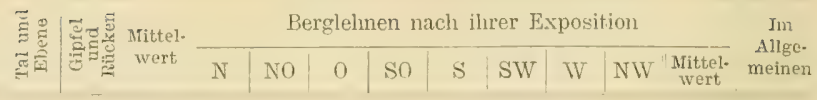

Syringa vulgaris $I$.

a) intero Grenze

Min.

51

b) oldere Grenze

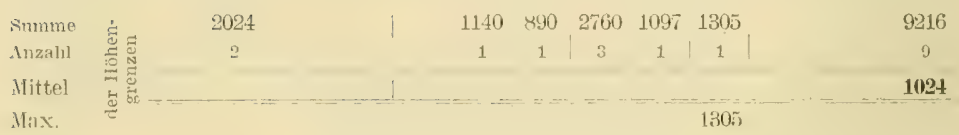

Simbucus racemusal $\mathrm{l}_{\text {. }}$.

a) untere Grenze

Summe
Muzal1
Mittel
Win.

b) obere Girmze

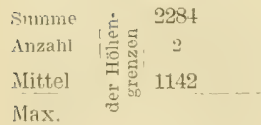

481) $\quad 2193$

1

180

\begin{tabular}{|c|c|c|c|}
\hline 2982 & 3136 & $1.12 t$ & 3062 \\
\hline 2 & 2 & 1 & 2 \\
\hline 1491 & 1568 & 1421 & $\frac{1531}{1599}$ \\
\hline
\end{tabular}

$1+178$ 10

1418 


\section{Bihar Gebirge.}

Cucurbéta 1819 m., Vlegyásza 1838 n., Vrf. Muntyeluj 1829 m., Vulkán $126 \pm$ m.

Berglehnen nach ihrer Exposition
Inı
MN

\section{Picea excelsa Lk.}

a) vereinzelt, untere Grenze

\begin{tabular}{|c|c|c|c|c|c|c|c|c|c|c|c|c|c|c|}
\hline Summe & & 2426 & 1110 & 3536 & 2288 & 1936 & 3471 & 3827 & 4107 & 954 & 5126 & 561 & 22270 & 25806 \\
\hline .Inzaln] & & 3 & 1 & + & 3 & 2 & 4 & 4 & 4 & 1 & 5 & 1 & 24 & sis \\
\hline itte & 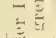 & $x 0 ! 5$ & 1110) & 884 & 763 & ! Wh & $x+3$ & $9 \%$ & 10107 & (5)1 & 1055 & 561 & 928 & 922 \\
\hline lin. & & & & & & & & & & & & 561 & & \\
\hline
\end{tabular}

b) rein, oder vorherrschend, untere Grenze

\begin{tabular}{|c|c|c|c|c|c|c|c|c|c|c|c|c|c|c|}
\hline Summe & & 1620 & 1150 & 2770 & 7912 & 2378 & 1880 & 10012 & 8319 & 5258 & $|4126|$ & | 2995 | & 42880 & 45650 \\
\hline Anzahl & & 2 & 1 & 3 & 8 & 2 & 2 & 9 & 7 & 4 & 4 & 3 & 39 & 42 \\
\hline ittel & & 8111. & 1151 & 929 & $9 \times !$ & 1184 & 940 & 1112 & $118 \mathrm{r}$ & 1.314 & 1032 & 994 & 1099 & 1089 \\
\hline lin. & & & & & 590 & & & & & & & & & \\
\hline
\end{tabular}

c) geschlossener Bestand, obere Grenze

simme

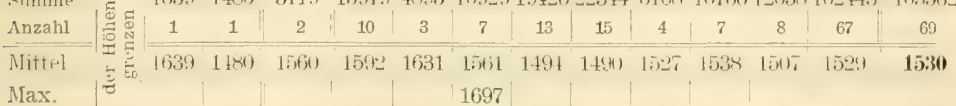

d) Raumbestand, obere Grenze (Waldgrenze)

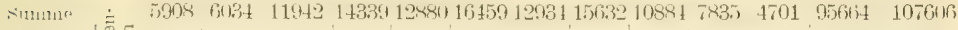
\begin{tabular}{l|c|cc|c|c|c|c|c|c|c|c|c|} 
Anzahl \\
\hdashline Mittel \\
Max.
\end{tabular} 


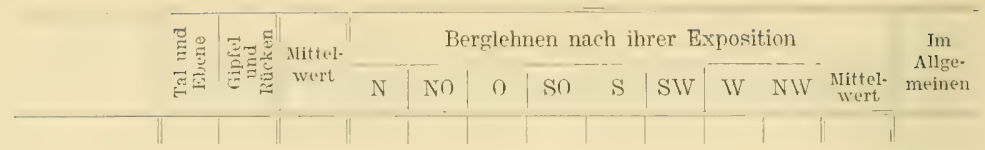

e) obere Baumgrenze

(Bäume mit 8 m. Gipfelhöhe.)

\begin{tabular}{|c|c|c|c|c|c|c|c|c|c|c|c|c|c|c|}
\hline Summe & & 4534 & $30 \div 0$ & 7479 & 9775 & 13849 & $1+9911$ & 13969 & $136+0$ & 7958 & 4833 & 6335 & 82311 & $89 \pi \div 0$ \\
\hline Anzahl & & 3 & 2 & 5 & 6 & 8 & 9 & 8 & 8 & 5 & 3 & 4 & 51 & 56 \\
\hline Mittel & -10 & 1.863 & 1510 & $143 \%$ & 1620 & 1612 & 1657 & $16 \geq 1$ & 1580 & 1592 & 1608 & $15 R 4$ & $161 t$ & $160 \%$ \\
\hline Max. & 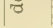 & & & & 1754 & & & & & & & & & \\
\hline
\end{tabular}

f) verkrüppelt, obere Grenze

\begin{tabular}{|c|c|c|c|c|c|c|c|c|c|}
\hline Sunme & $1 \dot{\nexists}$ & $17 \%$ & 3583 & 3461 & $31 \times 8$ & 1753 & $35 \geq 0$ & $1 \times 30$ & $19+12$ \\
\hline Anzahl & 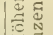 & 1 & 3 & 2 & 2 & 1 & 2 & 1 & 11 \\
\hline Mittel & 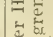 & 1777 & 1792 & 1730 & 1741 & 1753 & 1760 & 1830 & 1765 \\
\hline Max. & & & & & & & & 1830 & \\
\hline
\end{tabular}

\section{Abies pectinata DC. (A. alba Mill.)}

a) untere Grenze

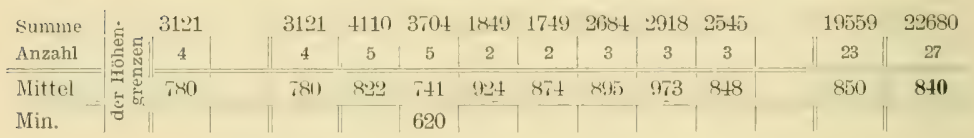

b) rein, oder vorherrschend, obere Grenze

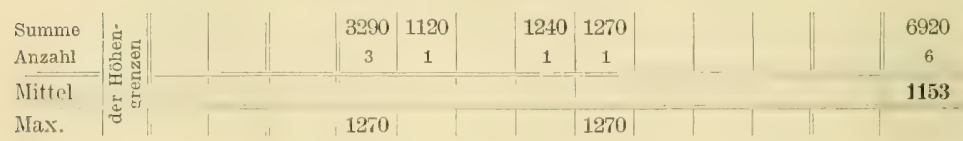

c) Baumform, obere Grenze

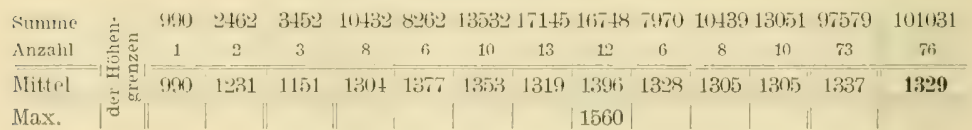

d) verkrüppelt, obere Grenze

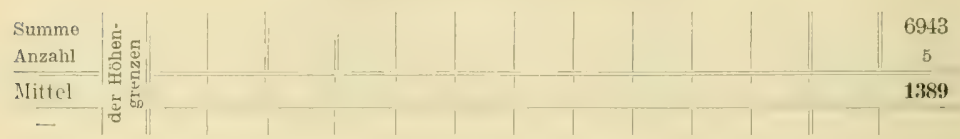




\begin{tabular}{|c|c|c|c|c|c|c|c|c|c|c|c|}
\hline \multirow{2}{*}{ 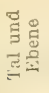 } & \multirow{2}{*}{ 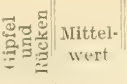 } & \multicolumn{9}{|c|}{ Berglehnen nach ihrer Exposition } & \multirow{2}{*}{$\begin{array}{c}\text { Im } \\
\text { Allge- } \\
\text { Het'in+? }\end{array}$} \\
\hline & & $\mathrm{N}$ & N11 & 11 & Si) & S & 511 & 11 & $\mathrm{NW}$ & "Mittel- & \\
\hline
\end{tabular}

\section{Pinus montana Mill.}

a) untere Grenze

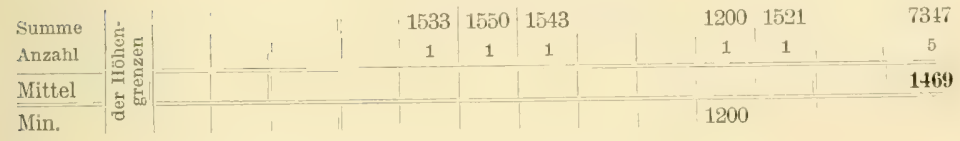

b) obere Grenze

\begin{tabular}{l|c|c|c|c|c|c|c} 
Summe \\
Anzah?
\end{tabular}

Juniperus Sabina L.

a) untere Grenze

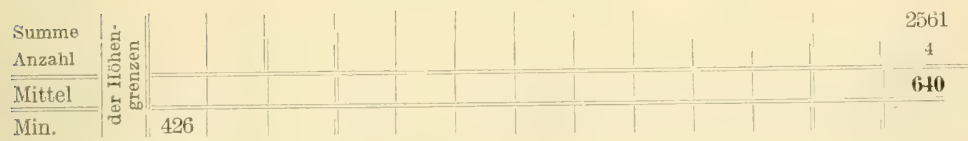

b) obere Grenze

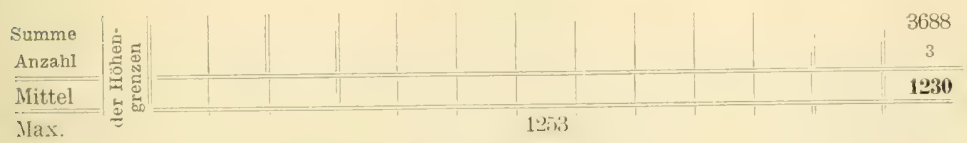

Fagus silvatiea $\mathbf{L}$.

a) vereinzelt, untere Grenze

\begin{tabular}{|c|c|c|c|c|c|c|c|c|c|c|c|c|}
\hline Summe & 2192 & 415 & 2607 & 1460 & 932 & 235 & 633 & 1063 & 168 & 1824 & $\begin{array}{c}6315 \\
\Upsilon \Upsilon 乛\end{array}$ & 8922 \\
\hline Anzalil & 10 & 2 & 12 & 7 & 4 & 1 & 3 & 3 & 1 & & & \\
\hline Mittel & 31 t & 218 & 317 & 305 & 333 & $23 \%$ & 211 & 351 & 168 & 304 & 253 & \\
\hline Min. & & & & 161 & & & & & & & & \\
\hline
\end{tabular}




\begin{tabular}{|c|c|c|c|c|c|c|c|c|c|c|c|}
\hline \multirow{2}{*}{ 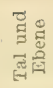 } & \multirow{2}{*}{\multicolumn{2}{|c|}{ 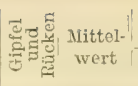 }} & \multicolumn{8}{|c|}{ Berglehnen nach ihrer Exposition } & \multirow{2}{*}{$\begin{array}{l}\text { Im } \\
\text { Allge- } \\
\text { meinen }\end{array}$} \\
\hline & & & $N$ & NO & 0 & $\mathrm{SO}$ & $\mathrm{S}$ & SW & W & NWV Mittel- & \\
\hline
\end{tabular}

b) rein, oder vorherrschend, obere Grenze

\begin{tabular}{l|c|c|c|c|c|c|c|c|c|c|} 
Summe & \multicolumn{1}{|c|}{$\begin{array}{c}1127 \\
\text { Anzahl }\end{array}$} \\
\hline Mittel & 581 & 1326 & 741 & 225 & 595 & 920 & 612 & & 6127 \\
\hline Min. & 5 & 2 & 4 & 1 & 1 & 1 & 2 & 1 & 17 \\
\hline
\end{tabular}

c) Bestandbildung im Allgemeinen, obere Grenze

\begin{tabular}{|c|c|c|c|c|c|c|c|c|c|c|c|c|c|c|}
\hline sumnete & & 5002 & MEs & 1196010 & $1 \leq n) 30$ & 8076 & 1001 & $161=8$ & 17160 & 1.093 & 19501 & $14 i 14$ & $1: 20733$ & 131393 \\
\hline Anzahl & है & 4 & 4 & 8 & 15 & 6 & 8 & 12 & 13 & 13 & 15 & 11 & 93 & 101 \\
\hline Mittel & 5 & 1250 & $1 \$ 14$ & 1322 & $121 i !$ & $13 \mathrm{tt}$ & 12.38 & $121 i 1$ & 1321 & 1315 & 1300 & $133 x$ & $139 x$ & 1301 \\
\hline Max. & & & 1499 & & & & & & & & & & & \\
\hline
\end{tabular}

$c_{1}$ ) obere Grenze der Bestandbildung als Waldgrenze

\begin{tabular}{|c|c|c|c|c|c|c|c|c|c|c|c|c|c|c|}
\hline Summo & & 2710 & 2890 & 5600 & 2710 & 4145 & 3929 & 1410 & 2713 & 4103 & 4147 & 9647 & 32804 & 38404 \\
\hline Anzahl & & 2 & 2 & 4 & 2 & 3 & $\hat{z}$ & 1 & 2 & 3 & 3 & 7 & 34 & 28 \\
\hline Mittel & $=0$ & $13 \pi 5$ & $1+45$ & 1400 & 1355 & $13 \times 2$ & 1310 & 1410 & 13.4 & Bitis & $13 \times 2$ & \multirow[t]{2}{*}{1378} & \multirow[t]{2}{*}{1306} & \multirow[t]{2}{*}{1378} \\
\hline Max. & & & 1499 & & & & & & & & & & & \\
\hline
\end{tabular}

$c_{2}$ ) obere Grenze der Bestandbildung, darüber Fichte

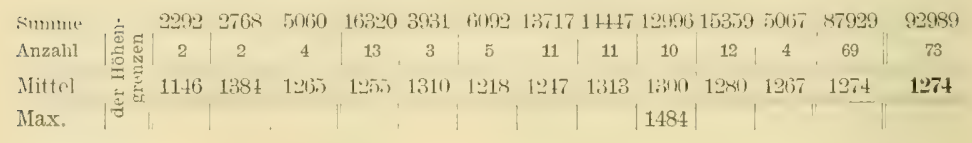

d) sporadisches Vorkommen im Fichtenbestand, obere Grenze

\begin{tabular}{|c|c|c|c|c|c|c|c|c|c|c|c|c|c|c|}
\hline Simmone & & 3561 & $3+5+8$ & 53) & 12054 & $3(1+3)$ & 13118 & 10905 & $1 \times 179$ & $5111 !$ & 17639 & $9 \pm 77$ & $947+1$ & $99 \%$ \\
\hline Anzalıl & 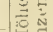 & 2 & 2 & 4 & 9 & 3 & 10 & S & 17 & 7 & 13 & 7 & 70 & $7 t$ \\
\hline Mittel & 可焉 & $1281)$ & 1324 & 1302 & 12339 & $130 \%$ & 1312 & 1363 & 1392 & $1: 3 \tilde{3}$ & 1357 & 13.1 & 1353 & 1351 \\
\hline Max. & 10 & & & & & & & & 1491 & & & & & \\
\hline
\end{tabular}

e) Strauchform, obere Grenze

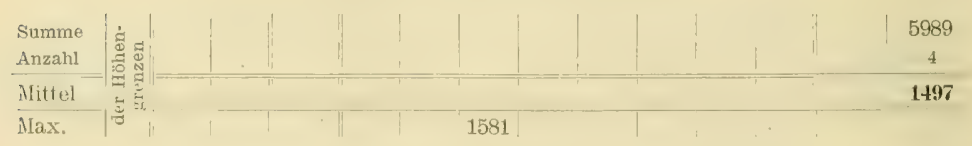




\begin{tabular}{|c|c|c|c|c|c|c|c|c|c|c|c|}
\hline \multirow{2}{*}{ 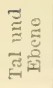 } & \multirow{2}{*}{ 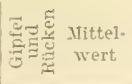 } & \multicolumn{9}{|c|}{ Berglelmen nach ihrer Exposition } & \multirow{2}{*}{$\begin{array}{l}\text { Im } \\
\text { Allghr- } \\
\text { meinen }\end{array}$} \\
\hline & & $\mathrm{N}$ & NO & 0 & $\mathrm{SO}$ & $\mathrm{S}$ & SIV & WT & NW & $\begin{array}{l}\text { Mittel- } \\
\text { wert }\end{array}$ & \\
\hline
\end{tabular}

\section{Quereus sessiliflora Nalish.}

a) rein oder vorherrschend, obere Grenze

\begin{tabular}{l|ccccccccccccccc}
$\begin{array}{l}\text { Summe } \\
\text { Anzahl }\end{array}$ \\
\begin{tabular}{l|c} 
Mittel \\
Max.
\end{tabular}
\end{tabular}

b) vereinzelt (Baumform), obere Grenze

\begin{tabular}{|c|c|c|c|c|c|c|c|c|c|c|c|c|c|}
\hline Surame & & 1805 & 1805 & 2486 & 2486 & 1658 & 6791 & $1753 t$ & 11981 & 4343 & ; 3399 & 49678 & 51483 \\
\hline Anzahl & $\frac{\pi}{0}$ & 3 & 3 & 3 & 3 & 2 & 8 & 19) & 14 & 5 & 3 & 57 & (n) \\
\hline Mittel & $\bar{c} \frac{\pi}{2}$ & 602 & 602 & 829 & 829 & 829 & 849 & 933 & 856 & 869 & 800 & 872 & 858 \\
\hline Iax. & & & & & & & & 1106 & & & & & \\
\hline
\end{tabular}

Quercus pedunculata Eluh. (Qu. Robur h.)

a) roin oder vorherrschend, obere Grenze

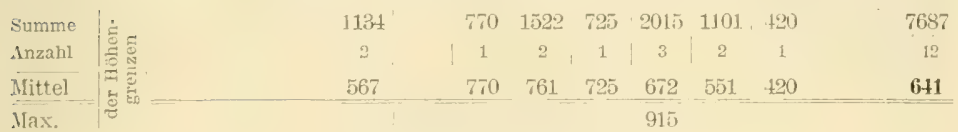

b) vereinzelt (Baumform), obere (irenze

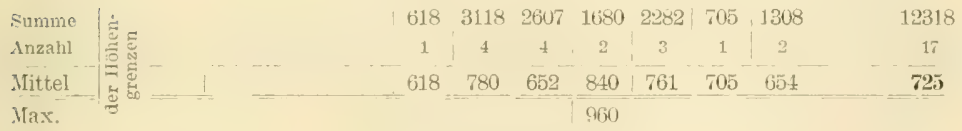

c) verkrüppelt, obere Grenze

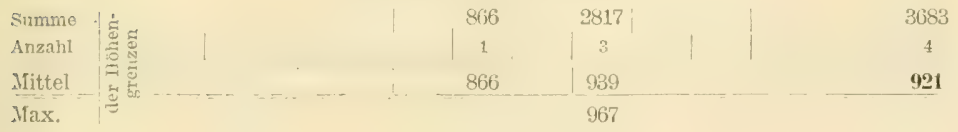




\begin{tabular}{|c|c|c|c|c|c|c|c|c|c|c|c|c|}
\hline \multirow{2}{*}{ 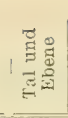 } & \multirow{2}{*}{\multicolumn{2}{|c|}{ 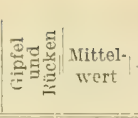 }} & \multicolumn{9}{|c|}{ Berglehnen nach ihrer Exposition } & \multirow{2}{*}{$\begin{array}{c}\text { Im } \\
\text { Allge- } \\
\text { meinen }\end{array}$} \\
\hline & & & $\mathrm{N}$ & No & 0 & so & $S$ & SW & W & NIV & $\begin{array}{l}\text { Mittel- } \\
\text { wert }\end{array}$ & \\
\hline
\end{tabular}

\section{Quercus lanuginosa Lam.}

obere Grenze

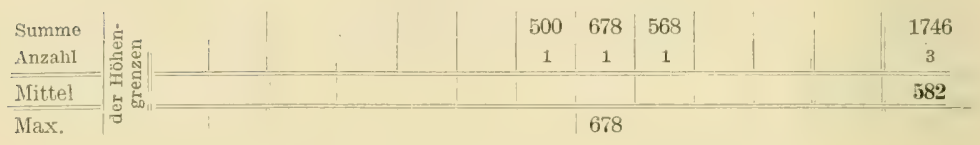

\section{Quereus couferta Kit.}

a) rein oder vorherrschend, obere Grenze

\begin{tabular}{|c|c|c|c|c|c|c|}
\hline $\begin{array}{l}\text { Summe } \\
\text { Anzahl }\end{array}$ & 这 & $\begin{array}{c}1524 \\
4\end{array}$ & $\begin{array}{c}412 \\
1\end{array}$ & $\begin{array}{c}744 \\
2\end{array}$ & $\begin{array}{c}450 \\
1\end{array}$ & $\begin{array}{c}3131 \\
8\end{array}$ \\
\hline Mittel & 至 & 391 & 412 & 37. & $\begin{array}{l}450 \\
450\end{array}$ & 391 \\
\hline
\end{tabular}

b) vereinzelt, obere Grenze

\begin{tabular}{|c|c|c|c|c|c|c|c|}
\hline Summe & 2253 & $79 \bar{i}$ & 1199 & 2098 & & 36 & 7208 \\
\hline Anzaht & J & 2 & 3 & 3 & 1 & 1 & 17 \\
\hline Mittel & 463 & 398 & 400 & 420 & 498 & $36 ;$ & 424 \\
\hline Max. & & & & 595 & & & \\
\hline
\end{tabular}

\section{Quercus Cerris L.}

vereinzelt, obere Grenze

\begin{tabular}{|c|c|c|c|c|c|c|c|}
\hline Summe & 1807 & 1190 & 1741 & 1944 & 705 & 774 & 8161 \\
\hline Anzahl & 3 & 2 & 3 & 3 & 1 & 1 & 13 \\
\hline Mittel & 603 & 595 & 580 & 648 & 705 & 774 & 628 \\
\hline Max. & & & & 837 & & & \\
\hline
\end{tabular}

Carpinus Betulus L.

a) bestandbildend, obere Grenze

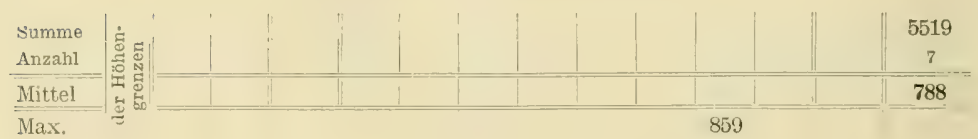




\begin{tabular}{l|l|l|l|l|l|l|l|l|l} 
Im \\
:
\end{tabular}

b) vereinzelt (Buumform), obere Grenze

\begin{tabular}{|c|c|c|c|c|c|c|c|c|c|c|c|c|}
\hline Summe & 1693 & 1693 & 4791 & 5700 & 6886 & 13398 & 12811 & 7514 & 16029 & 4735 & 71864 & 73557 \\
\hline Anzahl & 2 & 2 & 6 & 7 & 8 & 15 & 15 & 9 & 20 & 6 & 86 & 88 \\
\hline Mittel & $84 i$ & $x+\bar{i}$ & 769 & $\times 14$ & $x i j 1$ & 813 & nint & $\times 35$ & $x(11$ & $7 x: 4$ & 8,34 & $8: 36$ \\
\hline Max. & & & & & 1115 & & & & & & & \\
\hline
\end{tabular}

c) Strauchform, obere Grenze

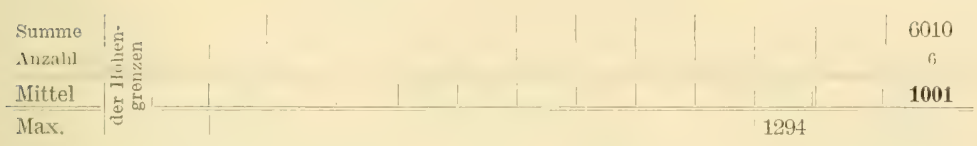

Corylus Arellana $\mathrm{L}_{\text {. }}$

\begin{tabular}{l|c|c|c|c|c|c|c|c|c|c|c|c|c|c}
\multicolumn{10}{c}{ obere Grenze } \\
$\begin{array}{l}\text { Summe } \\
\text { Anzahl }\end{array}$ \\
\hline Mittel \\
Max.
\end{tabular}

\section{Betula verrucosa Ehrl.}

a) bestandbildend, obere Grenze

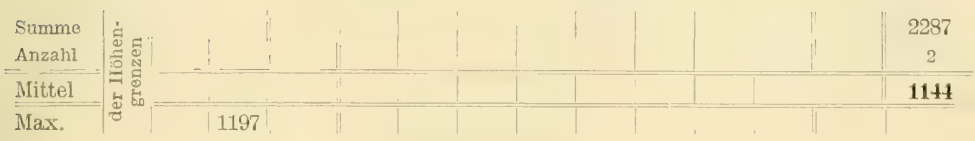

b) vereinzelt, obere Grenze

\begin{tabular}{|c|c|c|c|c|c|c|c|c|c|}
\hline Summe & 1330 & $1105^{\prime}$ & 2715 & 2507 & 1346 & 1489 & 1338 & 2667 & 14397 \\
\hline Anzathl & 1 & 1 & 2 & 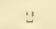 & 1 & 1 & 1 & 2 & 11 \\
\hline Mittr. & $13: 11$ & 1105 & $13 \pi 5$ & 1304 & $13 \mid t i$ & $1: 3$ & $1 ; \% 34$ & $1 ; 331$ & 1309 \\
\hline Max. & & & & 1422 & & & & & \\
\hline
\end{tabular}




\begin{tabular}{|c|c|c|c|c|c|c|c|c|c|c|c|c|c|}
\hline$\widetilde{d}$ & & & Tittel- & \multicolumn{9}{|c|}{ Berglehnen nach ihrer Exposition } & \multirow{2}{*}{$\begin{array}{l}\text { Im } \\
\text { Allge- } \\
\text { weinen }\end{array}$} \\
\hline हี: & & & wert. & $\mathrm{N}$ & No) & 0 & Si & S & $\mathrm{SW}$ & IV & $\mathrm{NIV}$ & $\begin{array}{l}\text { Mittel- } \\
\text { wert }\end{array}$ & \\
\hline
\end{tabular}

Aluus glutinosa Gårtn.

obere Grenze

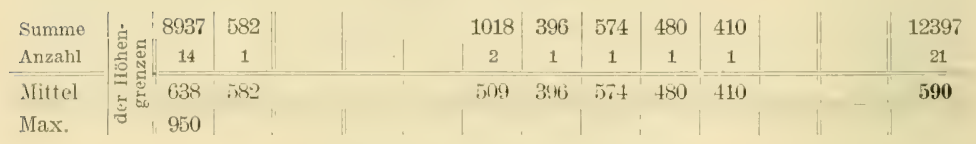

\section{Almus incana Willd.}

obere Grenze

\begin{tabular}{|c|c|c|c|c|c|c|c|c|c|}
\hline $\begin{array}{l}\text { Summe } \\
\text { Anzahl }\end{array}$ & 运 물 & $\left|\begin{array}{c}2064 \\
2\end{array}\right|$ & $\left|\begin{array}{c}2738 \\
3\end{array}\right|$ & $\begin{array}{c}2499 \\
3\end{array}$ & $\left|\begin{array}{c}2944 \\
3\end{array}\right|$ & $\begin{array}{c}3561 \\
4\end{array}$ & $\mid \begin{array}{c}1145 \\
1\end{array}$ & $\begin{array}{c}2660 \\
3\end{array}$ & $\begin{array}{c}17611 \\
19\end{array}$ \\
\hline Mittel & 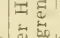 & 1033 & 913 & 833 & 981 & 890 & 1145 & 887 & 927 \\
\hline Max. & & & & & & & 1145 & & \\
\hline
\end{tabular}

\section{Aluus viridis $\mathrm{DC}$.}

a) untere Grenze

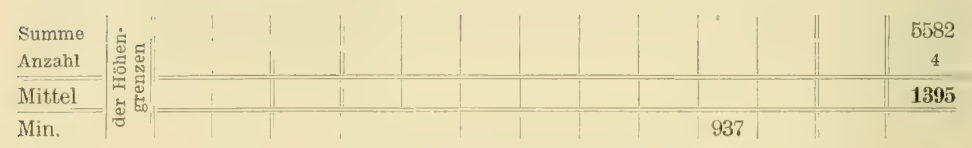

b) obere Grenze

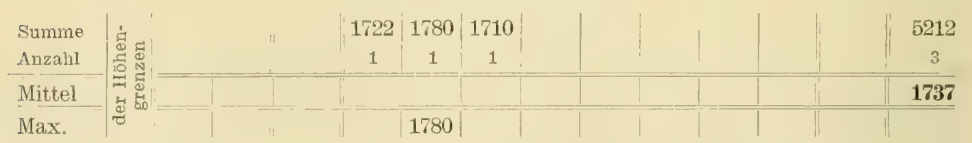

Salix alba L.

obere Grenze

\begin{tabular}{|c|c|c|c|c|c|c|c|c|c|c|}
\hline Summe & & 782 & 1930 & 5950 & 3789 & 2115 & 2262 & 4955 & 2693 & 24476 \\
\hline Anzahl & $\Xi$ & 1 & 2 & 6 & 4 & 2 & 3 & 5 & 3 & 26 \\
\hline Mittel & & 782 & 965 & 902 & 917 & 1057 & int & 991 & $8: 18$ & 941 \\
\hline $\operatorname{Max}$. & & & & & & 1200 & & & & \\
\hline
\end{tabular}




\begin{tabular}{|c|c|c|c|c|c|c|c|c|c|c|}
\hline \multirow{2}{*}{ 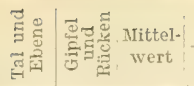 } & \multicolumn{9}{|c|}{ Berglehnen nach iher Exposition } & \multirow{2}{*}{$\begin{array}{l}\text { Im } \\
\text { Allge- } \\
\text { meinen }\end{array}$} \\
\hline & & No & & SO & S & SW & WV & NW & $\begin{array}{l}\text { Mittel- } \\
\text { wert }\end{array}$ & \\
\hline
\end{tabular}

\section{Populus tremula L.}

a) Baumform, obere Grenze

\begin{tabular}{|c|c|c|c|c|c|c|c|c|c|c|c|}
\hline Summe & & 2831 & 3792 & 1010 & 6627 & | 4137 | & | 3689 | & | $1012:$ & 1287 & 2409 & 26824 \\
\hline Anzahl & ¿ & 2 & 3 & 1 & 5 & 4 & 3 & 1 & 1 & 2 & 22 \\
\hline Mittel & है & $1 \mathrm{H} 1 \mathrm{t}$ & $1: 134$ & (1)141 & 13:; & 10,31 & 12310 & 1012 & $12 x: 1$ & 1.215 & 1210 \\
\hline Max. & 13 & & 1578 & & & & & & & & \\
\hline
\end{tabular}

b) Strauchform, obere Grenze

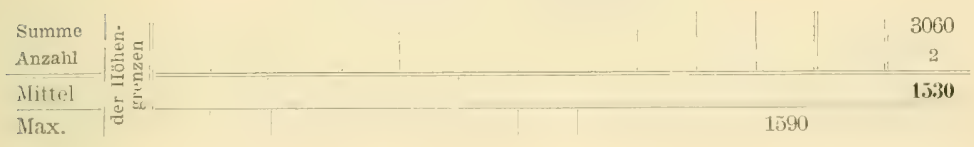

Ulmus montana With. (U. seabra Mill.)

a) mutere Grenze

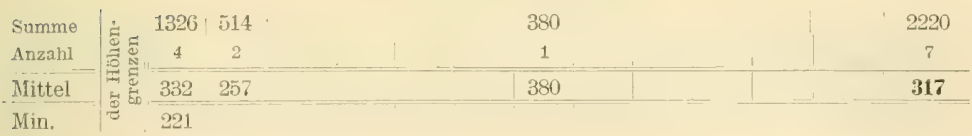

b) obere Grenze

\begin{tabular}{|c|c|c|c|c|c|c|c|c|c|c|}
\hline Summe & 1532 & 3130 & 1678 & 8129 & 2029 & 969 & 7306 & 9359 & 1005 & 355737 \\
\hline Anzahl & 2 & 4 & 2 & 9 & 2 & 1 & 7 & 10 & 1 & 38 \\
\hline . Mitted & ititj & sin & 8.31 & 648 & 1415 & โH:\} & $101+t$ & $13 i$ & $1(x)$, & 940 \\
\hline Max. & & & & & & & 1262 & & & \\
\hline
\end{tabular}

\section{Acer platanoides $\mathbf{I}$.}

a) Baumform, obere Grenze

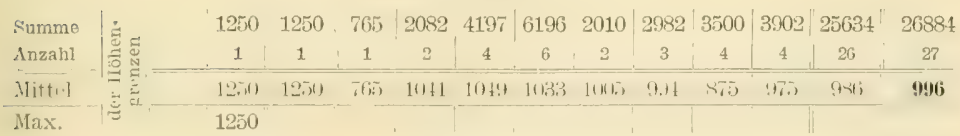




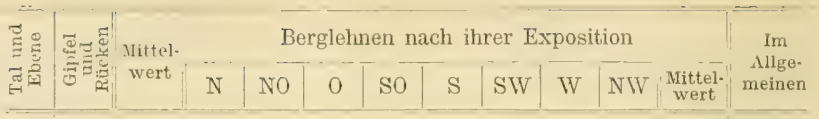

b) Strauchform, obere Grenze

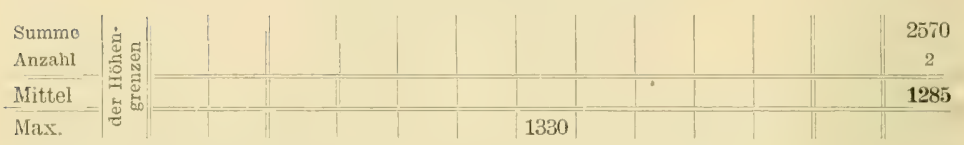

\section{Acer Pseudoplatanus I.}

Baumform, obere Grenze

\begin{tabular}{|c|c|c|c|c|c|c|c|c|c|c|c|c|}
\hline summe & $13 \pm 8$ & $13 \pm 8$ & 6512 & $6: 194$ & 8029 & 12491 & 6230 & $93^{2}+$ & .7178 & 11301 & 67462 & 68810 \\
\hline Anzahl & 1 & 1 & 5 & 5 & 6 & 10 & 5 & 7 & 5 & 8 & 51 & 52 \\
\hline Mittel & $13 \pm 8$ & $13 \pm 8$ & 1302 & 1299 & 1338 & 1219 & 1216 & 1332. & $1+30$ & 1400 & 1323 & 1323 \\
\hline Max. & & & & & & & & & 1585 & & & \\
\hline
\end{tabular}

\section{Acer eampestre $\mathbf{L}$.}

a) Baumform, obere Grenze

\begin{tabular}{|c|c|c|c|c|c|c|c|c|c|c|c|c|c|c|}
\hline Summe & & 3986 & 1303 & 5289 & 778 & 3809 & 1890 & 4974 & 1593 & 1711 & 1826 & 1380 & 17961 & 23250 \\
\hline Anzaln! & & 6 & 2 & 8 & 1 & 5 & 2 & 7 & 2 & 3 . & 3 & 2 & 25 & 33 \\
\hline Mittel & & $66+t$ & ties 1 & ti61 & 768 & 762 & 915 & 711 & 797 & 570 & 1009 & 690 & 718 & 705 \\
\hline Max. & & & & & & & 1090 & & & & & & & \\
\hline
\end{tabular}

b) obere Grenze, im Allgemeinen

\begin{tabular}{|c|c|c|c|c|c|c|c|c|c|c|c|c|c|c|}
\hline Fimme & & $34 \times 6$ & 2089 & 6275 & $T 7 R$ & 3809 & 1890 & 6012 & 1593 & 1711 & 18.6 & 1380 & 18999 & 25274 \\
\hline Anzahl & 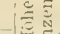 & i) & 3 & 9 & 1 & 5 & 2 & 8 & 2 & 3 & 3 & 2 & 26 & (2) \\
\hline Mittel & 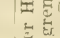 & 664 & $7 t 3$ & 1397 & 778 & 762 & 945 & 752 & 797 & 570 & 609 & 600 & 731 & 722 \\
\hline Max. & & & & & & & 1090 & & & & & & & \\
\hline
\end{tabular}

\section{Fraxinus excelsior $\mathbf{L}$.}

Baumform, obere Grenze

\begin{tabular}{|c|c|c|c|c|c|c|c|c|c|c|c|c|c|c|}
\hline Sinume & & $17(x)$ & $1: 3(10)$ & $2 x)(x)$ & $466 ?$ & 5,991 & 5706 & (\$313 & 5602 & 6.157 & 11060 & 6.849 & 55670 & xखivo \\
\hline Anzalil & & 2 & 1 & 3 & 5 & 6 & 6 & 9 & 5 & 6 & 11 & 7 & 55 & 58 \\
\hline Mittil & $\overrightarrow{5}$ & 8,0 & $12+10$ & $96 \overline{1}$ & $93+$ & 999 & 5 & 11935 & $112 t$ & 1076 & $10(0) 5$ & 978 & 1012 & 1010 \\
\hline Max. & & & & & & 1350 & & & & & & & & \\
\hline
\end{tabular}




\begin{tabular}{|c|c|c|c|c|c|c|c|c|c|c|c|c|}
\hline \multirow{2}{*}{ 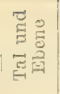 } & \multirow{2}{*}{ 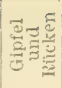 } & \multirow{2}{*}{$\begin{array}{l}\text { Alittel- } \\
\text { wert }\end{array}$} & \multicolumn{9}{|c|}{ Berglehnen mach ihrer Exposition } & $\mathrm{Im}$ \\
\hline & & & $\mathrm{N}$ & No & 0 & $\mathrm{SO}$ & S & SIV & WV & NW & $\begin{array}{l}\text { Mittel- } \\
\text { wert }\end{array}$ & meinen \\
\hline
\end{tabular}

\section{Sorbus aucuparia L.}

a) Baumform, obere Grenze

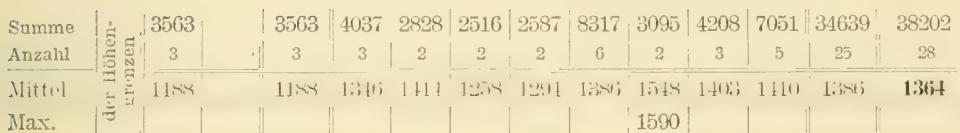

b) Strauchform, obere Grenze

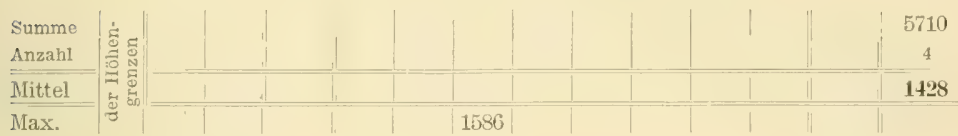

Crataegus monogyna Jacq. und oxyacantha $\mathbf{L}$.

obere Grenze

\begin{tabular}{|c|c|c|c|c|c|c|c|c|c|c|}
\hline $\begin{array}{l}\text { Summe } \\
\text { Anzahl }\end{array}$ & 总 & $\begin{array}{c}2178 \\
2\end{array}$ & $\begin{array}{c}1010 \\
1\end{array}$ & $\begin{array}{c}6705 \\
6\end{array}$ & $\begin{array}{c}4398 \\
4\end{array}$ & $\begin{array}{c}8521 \\
8\end{array}$ & $\begin{array}{c}2031 \\
2\end{array}$ & $\begin{array}{c}1115 \\
1\end{array}$ & $\left|\begin{array}{c}1060 \\
1\end{array}\right|$ & $\begin{array}{c}270.18 \\
25\end{array}$ \\
\hline Nittel & 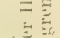 & 108.9 & li) 10 & $111 \%$ & los. & liffis & 1016 & (111) & 100 ก & $108^{\circ}$ \\
\hline Iax. & & & & & & 1219 & & & & \\
\hline
\end{tabular}

Syringa Josikaea Jacy. fil.

a) untere Grenze

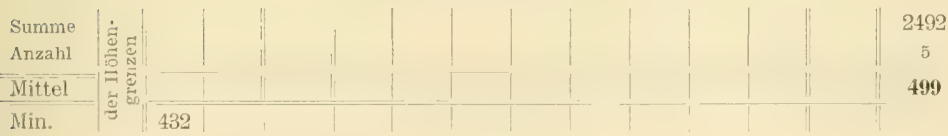

b) obere Grenze:

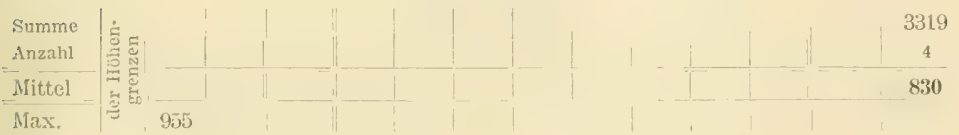




\begin{tabular}{|c|c|c|c|c|c|c|c|c|c|c|c|}
\hline \multirow{2}{*}{ 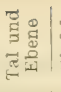 } & \multirow{2}{*}{ 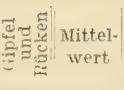 } & \multicolumn{9}{|c|}{ Berglehnen nach ihrer Exposition } & \multirow{2}{*}{$\begin{array}{c}\text { Im } \\
\text { Allge- } \\
\text { meinen }\end{array}$} \\
\hline & & N & NO & 0 & SO & $\$$ & SWV & WV & NW & $\begin{array}{c}\text { Mittel- } \\
\text { wert }\end{array}$ & \\
\hline
\end{tabular}

Syringa vulgaris $\mathbf{L}$.

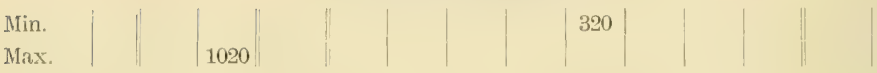

Sambucus racemosi $\mathbf{f}$.

a) intere Grenze

Min. | || 686

b) obere Grenze

\begin{tabular}{l|l|c|ccc|c|c|c|c|c||c} 
Summe \\
Anzahi
\end{tabular}




\section{Ungarisches Mittelgebirge.}

Mátra (Kékestetô) 1010 m., Bükk (Bálvúny) 957 m., Börzsönyi hegyek (Csóványos) 939 m., Bakony (Kốrös) $713 \mathrm{~m}$., Pilis $757 \mathrm{~m}$. , Vértes (Gerecse) $633 \mathrm{~m}$.

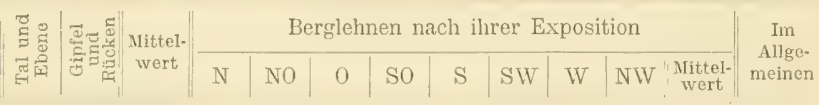

\section{Fagus silratica I.}

a) vereinzelt, untere Grenze

\begin{tabular}{|c|c|c|c|c|c|c|c|c|c|c|c|c|c|}
\hline Summe & & 2195 & 840 & 3035 & 1704 & 1109 & 1160 & 197 & 305 & 535 & 908 & 5918 & 8953 \\
\hline Anzah1 & & 9 & 3 & 12 & 7 & 4 & 3 & 1 & 1 & 2 & 4 & 22 & \\
\hline $\begin{array}{l}\text { Iittel } \\
\text { lin. }\end{array}$ & & $\begin{array}{l}24 \\
116\end{array}$ & 280 & 23 & 43 & 2.7 & 348 & 147 & $: 305$ & 248 & 24 & $2 f(5)$ & \\
\hline
\end{tabular}

b) rein, oder vorherrschend, untere Grenze

\begin{tabular}{|c|c|c|c|c|c|c|c|c|c|c|c|c|}
\hline Summe & 748 & 748 & 1773 & 1151 & 938 & 341 & 373 & 340 & 300 & 101 & 6267 & 7015 \\
\hline Inzahl & 3 & 3 & 7 & 4 & 3 & 1 & 1 & 1 & 1 & 4 & 22 & 25 \\
\hline $\begin{array}{l}\text { iittel } \\
\text { lin. }\end{array}$ & $\begin{array}{l}349 \\
116\end{array}$ & 24 & 453 & $3 \times !$ & 313 & $3+1$ & 363 & 340 & 301 & stis & 2 & \\
\hline
\end{tabular}

\section{Quereus sessiliflora Salisb.}

a) rein, oder vorherrschend, obere Grenzè

\begin{tabular}{|c|c|c|c|c|c|c|c|c|c|c|c|c|}
\hline Summe & , & $\mid 5615$ & 5615 & 989 & 1154 & 3086 & 5026 & 6145 & 8801 & 3618 & 28819 & 34434 \\
\hline Anzainl & 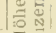 & 8 & 8 & 2 & 2 & 5 & 7 & 9 & 13 & 6 & 44 & 52 \\
\hline Mittel & $\bar{t}$ & 702 & 702 & 495 & 577 & 617 & 718 & 683 & 677 & 603 & $6 \overline{5} 5$ & 662 \\
\hline Max. & $=$ & 899 & & & & & & & & & & \\
\hline
\end{tabular}




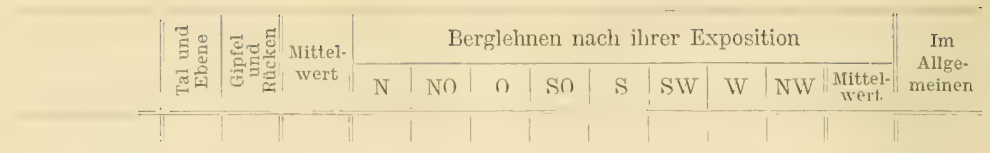

b) vereinzelt (Baumform), obere Grenze

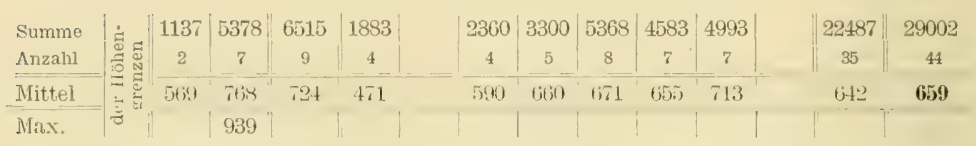

c) verkriippelt

Max.

$940 !$

\section{Quereus pedunculata Ehrh. (Qu. Robur $\left.\mathbf{I}_{\text {. }}\right)$}

a) rein, oder vorherrschend, obere Grenze

\begin{tabular}{|c|c|c|c|c|c|c|c|c|}
\hline Summe & 630 & 630 & 410 & 1367 & 1114 & 1276 & 4167 & 4797 \\
\hline Anzahl & 2 & 2 & 1 & 4 & 2 & 3 & 10 & 12 \\
\hline Mittel & 315 & 315 & 411) & 312 & 557 & $42 \pi$ & 417 & 400 \\
\hline Max. & & & & & 734 & & & \\
\hline
\end{tabular}

b) vereinzelt, obere Grenze

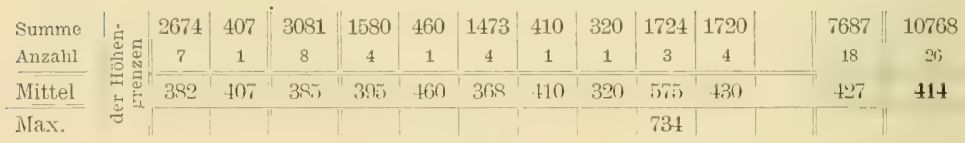

\section{Quereus lanuginosa Lam.}

a) vorherschend, obere Grenze

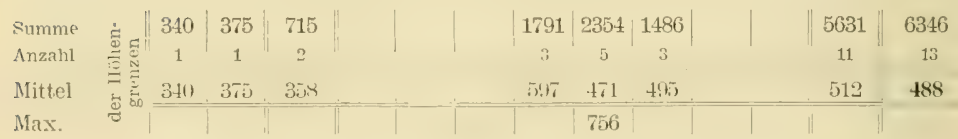

b) vereinzelt, obere Grenze

\begin{tabular}{|c|c|c|c|c|c|c|c|c|c|c|c|c|}
\hline Summe & 1082 & 3878 & $\sin 60$ & 321 & 1347 & 1946 & 3600 & 2041 & 832 & 364 & $10 \div 04$ & 15364 \\
\hline Anzahl & 3 & 8 & 11 & 1 & 3 & 3 & 7 & 3 & 2 & 1 & 90 & 31 \\
\hline $\begin{array}{l}\text { Mittel } \\
\text { Max. }\end{array}$ & 361 & 185 & 451 & 321 & 432 & 649 & $\begin{array}{l}514 \\
756\end{array}$ & 681 & 416 & 364 & 520 & 496 \\
\hline
\end{tabular}




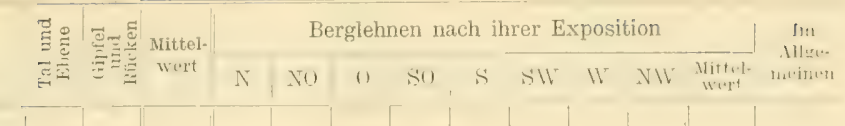

Quereus Cerris 1.

\begin{tabular}{l|c|c|c|c|c|c|c|c|c|c|c|c|c|c|} 
a) rorherschend, obere Grenze \\
$\begin{array}{l}\text { Summe } \\
\text { Anzahl }\end{array}$ \\
Mittel \\
Max.
\end{tabular}

b) vereinzelt (Baumform), obere Grenze

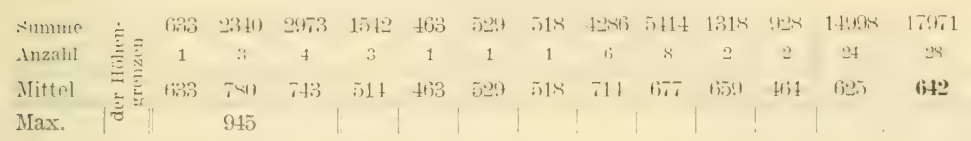

c) verkriippelt

$\operatorname{Max}$.

\section{Capinus, Betulus L.}

a) bestandbildend, obere Grenze

\begin{tabular}{l|c|c|c|c|c|c|c|c|c|c|} 
Summe \\
Anzahl \\
\hline \hline Mittel
\end{tabular}

b) vereinzelt (Baumform), obere Grenze

\begin{tabular}{|c|c|c|c|c|c|c|c|c|c|c|c|c|c|}
\hline Summe & & 839 & 6169 & 7008 & 806 & 515 & 827 & 1581 & 1748 & 2586 & 2155 & 10218 & 17226 \\
\hline Anzahl & & 1 & 7 & 8 & 1 & 1 & 1 & 2 & 2 & 3 & 3 & 13 & 21 \\
\hline Mittin & & $x, 39$ & sil & sit; & Sinj & 515 & $x \geq$ & $7: 11$ & n.t & Nig & 718 & $i x i$ & $8 \div 0$ \\
\hline Iax. & & & $9 \overline{5}$ & & & & & & & & & & \\
\hline
\end{tabular}

c) verkrüppelt, obere Grenze

\begin{tabular}{l|c|c|c|c|c|c|c|}
$\begin{array}{l}\text { Summe } \\
\text { Anzaht }\end{array}$ \\
\hline Mittel \\
\hline Max.
\end{tabular} \mid \begin{tabular}{c|c|c|c|c|c|c|}
3685 \\
0
\end{tabular}




\begin{tabular}{|c|c|c|c|c|c|c|c|c|c|c|c|c|}
\hline & Nittel- & \multicolumn{10}{|c|}{ Berglehnen nach ihrer Exposition } & \\
\hline 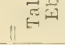 & wert & $\mathrm{N}$ & & NO & 0 & So & $S$ & SW & WV & NW & $\begin{array}{l}\text { Mitter- } \\
\text { wert }\end{array}$ & meinen \\
\hline
\end{tabular}

\section{Alnus glutinosa Gärtu.}

obere Grenze

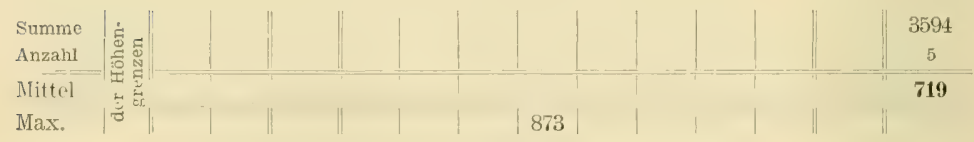

Ulmus montana With. (U. seabra Mill.)

intere Grenze

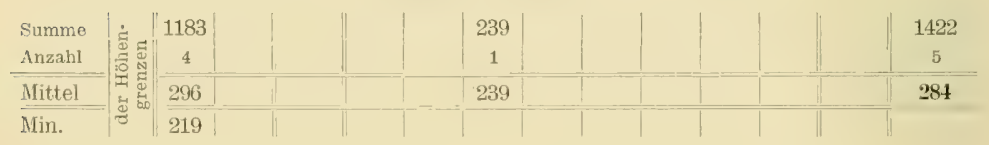

Acer campestre L.

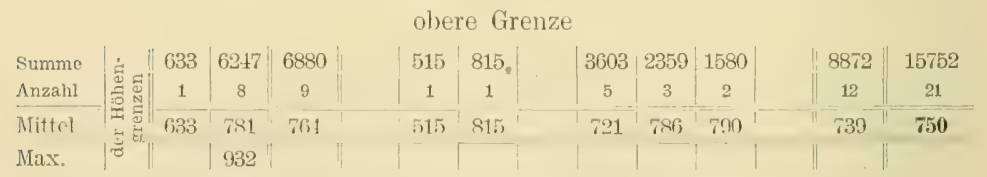

Acer tataricum $\mathbf{L}$.

obere Grenze

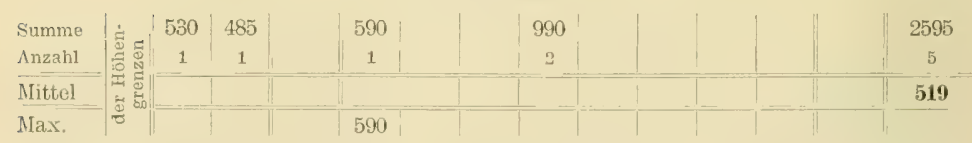

Fraxinus excelsior L.

Baumform, obere Grenze

\begin{tabular}{|c|c|c|c|c|c|c|c|c|c|}
\hline Simmine & 1261 & $\operatorname{tin} 60$ & $81: 21$ & 3162 & 1835 & 891 & 600 & 6391. & 14512 \\
\hline Anzahl & 2 & 8 & 10 & 4 & 2 & 1 & 1 & 8 & 18 \\
\hline Mittel & 631 & $\times \tilde{5}$ & 812 & $7(6) 5$ & 918 & $89 t$ & 600 & 799 & 806 \\
\hline $\operatorname{Max}$. & & & & 950 & & & & & \\
\hline
\end{tabular}




\begin{tabular}{|c|c|c|c|c|c|c|c|c|c|c|c|c|}
\hline \multirow{2}{*}{ 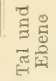 } & \multirow{2}{*}{ 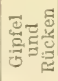 } & \multirow{2}{*}{$\begin{array}{l}\text { Mittel- } \\
\text { wert }\end{array}$} & \multicolumn{9}{|c|}{ Berglehnen nach ihrer Exposition } & \multirow{2}{*}{$\begin{array}{c}\text { Im } \\
\text { Allge- } \\
\text { meinen }\end{array}$} \\
\hline & & & $\mathrm{N}$ & No & 0 & so & S & SW & W & NIV & $\begin{array}{l}\text { Mittel- } \\
\text { wert }\end{array}$ & \\
\hline
\end{tabular}

\section{Fraxiuns Ormus L.}

\begin{tabular}{|c|c|c|c|c|c|c|c|c|c|c|c|c|c|}
\hline Summe & & 537 & 1317 & 1854 & 529 & 475 & 1175 & 473 & 3623 & 1873 & 1785 & 9933 & 11787 \\
\hline Anzahl & & 1 & 2 & 3 & 1 & 1 & 2 & 1 & 7 & 3 & 3 & 18 & 21 \\
\hline Mlittel & & 537 & (⿻5口)! & 618 & i) & $42 \pi$ & $\pi 4 x$ & 473 & i) 16 & 162t & 595 & 522 & 561 \\
\hline Max. & \% & & & & & & & & & 808 & & & \\
\hline
\end{tabular}

\section{Sorbus torminalis Crantz.}

obere Grenze

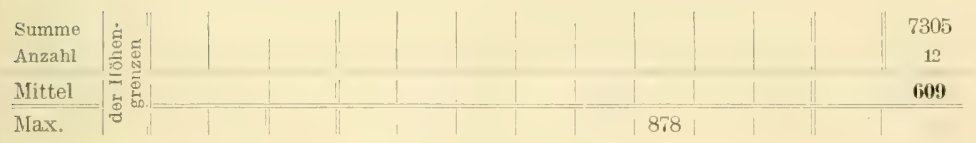

Prunus Mahaleb $\mathbf{L}$.

obere Grenze

\begin{tabular}{l|l|c|c|c|c|c|c|c|c|c|} 
Summe & 548 & 1541 & 2089 & 548 & 529 & $1156^{2}$ & 780 & & 3013 & 5102 \\
Anzahl & Mittel \\
Max. & 1 & 2 & 3 & 1 & 1 & 2 & 1 & 5 & 8 \\
\hline
\end{tabular}

\section{Cotimus Coggygria Seop.}

obere Grenze

$\operatorname{Max}$ 


\section{Inselgebirge.}

Papuk 953 m., Mecsek (Zengó) 682 m., Fruska Gora 539.

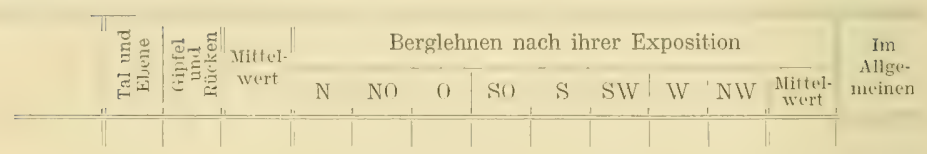

Abies pectimata DC. (A. alba Mill.)

untere Grenze

Min. ||$\quad|"| 321 \mid$

Figus silvatica $L$.

\begin{tabular}{l|c|c|c|c|c|c|c|c|c|c|c|c|c|c|} 
untere Grenze \\
$\begin{array}{l}\text { Summe } \\
\text { Anzalil }\end{array}$ \\
\hline Mittel \\
Min.
\end{tabular}

\section{Quereus sessilillora Salisb.}

a) rein, oder vorherrschend, obere Grenze

\begin{tabular}{|c|c|c|c|c|c|c|c|c|c|c|c|}
\hline Summe & 318 & 318 & 981 & $8 \pm 1$ & 1856 & 4982 & 2933 & 1502 & 677 & $\mid 13772$ & 14090 \\
\hline . Iuzalıl & 1 & 1 & 2 & 2 & 3 & 111 & f) & 4 & 2 & : & 3) \\
\hline Mittel & $31 \times$ & $31 x$ & $1: 11$ & 121 & filst & $1 ! K$ & $f \times ! !$ & 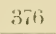 & 330 & (iii) & 470 \\
\hline Max. & & & & & 815 & 815 & & & & & \\
\hline
\end{tabular}

b) vereinzelt (Baumform), obere Grenze

\begin{tabular}{|c|c|c|c|c|c|c|c|c|c|c|c|}
\hline $\begin{array}{l}\text { Summe } \\
\text { Anzahl }\end{array}$ & $\begin{array}{c}3163 \\
6\end{array}$ & $\begin{array}{c}3163 \\
6\end{array}$ & $\begin{array}{c}1862 \\
4\end{array}$ & $\begin{array}{c}841 \\
2\end{array}$ & $\begin{array}{c}1520 \\
2\end{array}$ & $\begin{array}{c}2328 \\
4\end{array}$ & $\begin{array}{c}2697 \\
4\end{array}$ & $\begin{array}{c}707 \\
2\end{array}$ & $\begin{array}{c}800 \\
2\end{array}$ & $\begin{array}{c}10755 \\
20\end{array}$ & $\begin{array}{c}13918 \\
26\end{array}$ \\
\hline Nittel & 52. & $\therefore 27$ & Itit) & $1 \geq 1$ & (6i) & int & $15 t$ & 3ist & $f(x)$ & $53 x$ & 53.3 \\
\hline Max. & & & & & (815) & 815 & (815) & & & & \\
\hline
\end{tabular}


Quercus pedunculata Ehrh. (Qu. Robur L.)

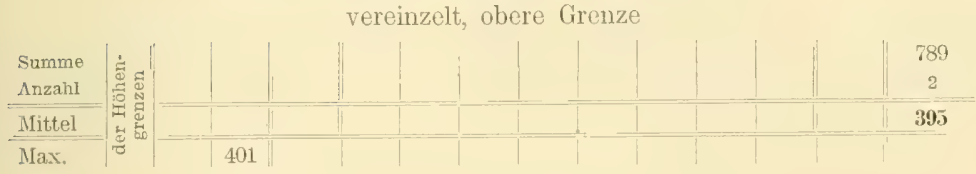

\section{Quereus lanuginosa Lam.}

obere Grenze

\begin{tabular}{|c|c|c|c|c|c|c|c|c|c|}
\hline $\begin{array}{l}\text { Summe } \\
\text { Anzahl }\end{array}$ & 苛 & $\begin{array}{c}379 \\
1\end{array}$ & $\begin{array}{c}553 \\
1\end{array}$ & $\begin{array}{c}748 \\
2\end{array}$ & $\begin{array}{c}841 \\
2\end{array}$ & $\begin{array}{c}340 \\
1\end{array}$ & $\begin{array}{c}811 \\
2\end{array}$ & $\begin{array}{c}5 \tilde{3} 3 \\
1\end{array}$ & $\begin{array}{c}4225 \\
10\end{array}$ \\
\hline Mittel & F & & & & & & & & 423 \\
\hline Max. & & & 553 & & & & & 553 & \\
\hline
\end{tabular}

\section{Quereus Cerris L.}

obere Grenze

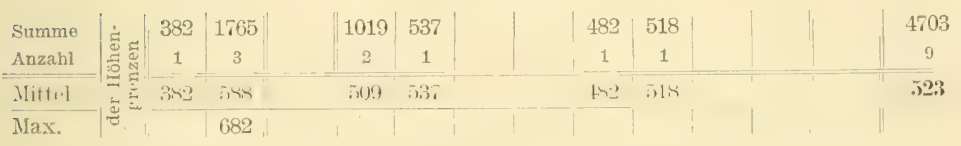

\section{Castanea sativa Mill.}

untere Grenze
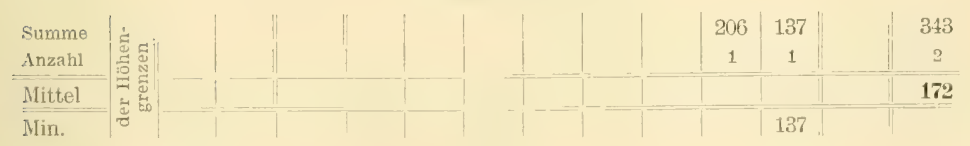

Carpinus Betulus L.

a) bestandbildend, obere Grenze

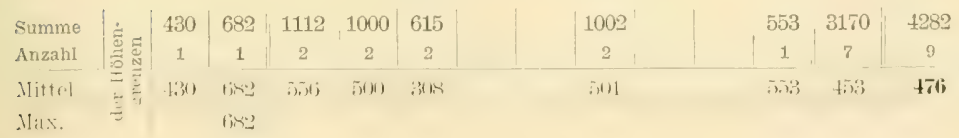




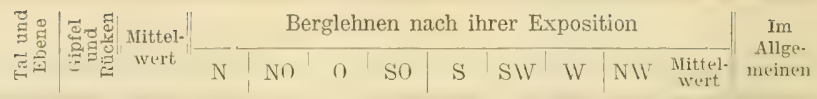

b) vereinzelt, obere Grenze

\begin{tabular}{|c|c|c|c|c|c|c|c|c|c|}
\hline Summe & & 2746 & 2746 & 331 & 2391 & 482 & 383 & 3590 & 6336 \\
\hline Anzahl & 施 & 5 & 5 & 1 & 4 & 1 & 1 & 7 & 12 \\
\hline Mittel & $\Rightarrow$ & 549 & 519 & 331 & 549 & $4 \times 2$ & $3 \times 3$ & 513 & 528 \\
\hline Max. & & & & & 953 & & & & \\
\hline
\end{tabular}

Fraxinus 0rnus $\mathbf{I}$.

obere Grenze

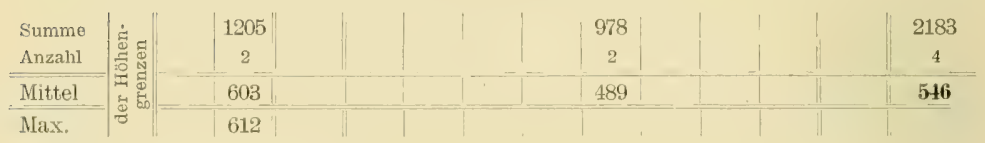




\section{Hiigelland beim Bilatonsee.}

Grösste Erhebung $315 \mathrm{~m}$.

至

Fagus silvatica $\mathbf{I}_{\text {. }}$.

untere Grenze

$$
\begin{aligned}
& \text { Summe } \\
& \text { Anzaht } \\
& \text { Mittel }
\end{aligned}
$$




\section{Ungarische Alpen.}

Irottkố $883 \mathrm{~m}$. , Rozália $746 \mathrm{~m}$.

\begin{tabular}{|c|c|c|c|c|c|c|c|c|c|c|c|c|}
\hline \multirow{2}{*}{ 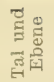 } & \multirow{2}{*}{ 혬제 } & \multirow{2}{*}{$\begin{array}{l}\text { Mittel } \\
\text { wert }\end{array}$} & \multicolumn{9}{|c|}{ Berglehnen nach ihrer Exposition } & \multirow{2}{*}{$\begin{array}{c}\text { Im } \\
\text { Allge- } \\
\text { meinen }\end{array}$} \\
\hline & & & $\mathrm{N}$ & NO & 0 & so & S & SW & W & NW & & \\
\hline
\end{tabular}

Picen excelsa Lk.

a) vereinzelt, untere Grenze

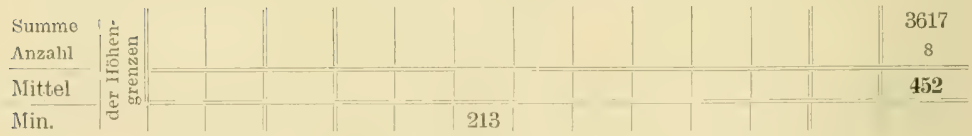

b) rein, oder vorherrschend, untere Grenze

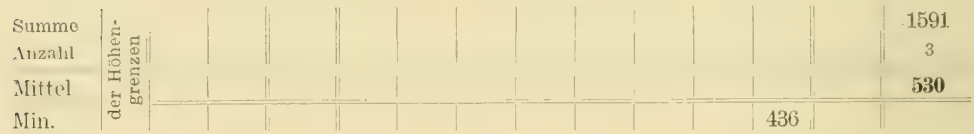

Abies pectimata 100. (A. alba Mill.)

a) vereinzelt, untere Grenze

\begin{tabular}{l|c|c||c|c|c|c|c|c|c|c|c}
$\begin{array}{l}\text { Summe } \\
\text { Anzahl }\end{array}$ \\
\hline $\begin{array}{l}\text { Mit.tel } \\
\text { Nin. }\end{array}$
\end{tabular}

b) rein, oder vorherrschend, untere Grenze

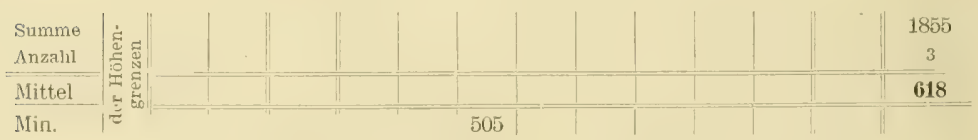




\begin{tabular}{|c|c|c|c|c|c|c|c|c|c|c|c|}
\hline \multirow{2}{*}{ 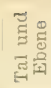 } & \multirow{2}{*}{ 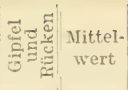 } & \multicolumn{9}{|c|}{ Berglehnen nach ihrer Exposition } & \multirow{2}{*}{$\begin{array}{c}\text { Im } \\
\text { Allge- } \\
\text { meinen }\end{array}$} \\
\hline & & $\mathrm{N}$ & NO & 0 & So & $D$ & SW & WV & NWV & $\begin{array}{c}\text { Mittel- } \\
\text { wert }\end{array}$ & \\
\hline
\end{tabular}

\section{Pinus silrestris $\mathbf{L}$.}

\begin{tabular}{|c|c|c|c|c|c|c|c|c|c|c|c|c|}
\hline Summe & & 620 & 388 & 1059 & 630 & 1003 & 434 & 1569 & 1436 & 466 & 6597 & 7217 \\
\hline Anzahl & 造 & 3 & 2 & 3 & 2 & 3 & 2 & 5 & 4 & 2 & 21 & 24 \\
\hline $\begin{array}{l}\text { Mittel } \\
\text { Min. }\end{array}$ & 可 & $\begin{array}{l}3413 \\
185\end{array}$ & 191 & (inis & 31. & 331 & $21 \%$ & $31 t$ & $3 \pi 9$ & 293 & $31 . t$ & 301 \\
\hline
\end{tabular}

Larix europaca DC. (L. Mecidua Mill.)

untere Grenze

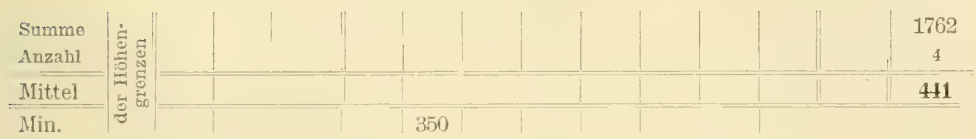

\section{Fagus silvatica $\mathbf{L}$.}

a) vereinzelt, untere Grenze

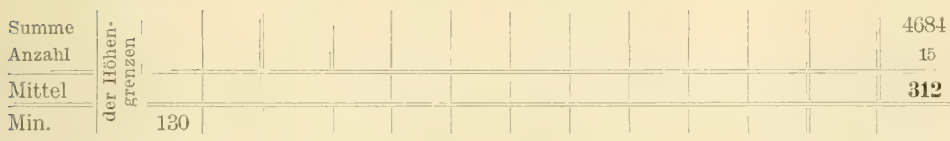

b) rein, oder vorherrschend, untere Grenze

\begin{tabular}{|c|c|c|c|c|c|c|c|c|c|c|}
\hline Summe & $\dot{\dot{\theta}}$ & 180 & 841 & 752 & 1026 & 1107 & 750 & 220 & 1431 & 6307 \\
\hline Anzahi & 욤 & 1 & 3 & 2 & 2 & 2 & 1 & 1 & 4 & 16 \\
\hline Mittel & 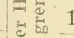 & 180 & 280 & 376 & 513 & 553 & 750 & 220 & 358 & 394 \\
\hline Min. & $\approx \| 1$ & 180 & & & & & & & & \\
\hline
\end{tabular}

\section{Quercus sessiliflora Salisb.}

a) rein, oder vorherrschend, obere Grenze

\begin{tabular}{l|l|l|c|c|c|c|c|c|c||c|}
$\begin{array}{l}\text { Summe } \\
\text { Anzahl }\end{array}$ \\
\hline Mittel
\end{tabular}




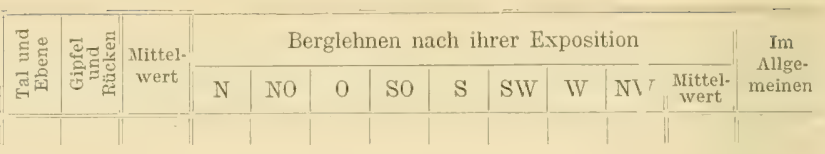

b) vereinzelt, obere Grenze

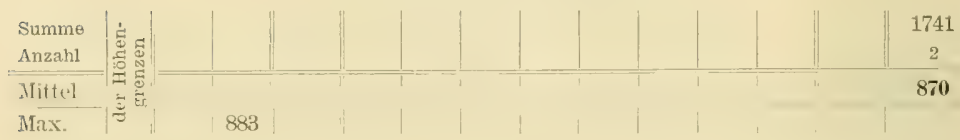

Almus ririlis DC.

mutere Grenze

\begin{tabular}{l|c|c|c|c|c|c|c|c} 
Summe \\
Anzahl
\end{tabular}




\section{Kroatische Alpen.}

Velebit 1760 m., Pljesevica (Szellin) 1657 m., Nagy-KKapella = Grosse Kapella (Biela Lasica) 1533 m., Risznyák 1528 m., Kis-Kapella = Kleine Kapella 1280 m., Uskok (Gorianc) $1181 \mathrm{~m}$., Ivanščica $1061 \mathrm{~m}$., Sljeme $1035 \mathrm{~m}$. , Zrinyi Hegrség = Zrinska gori = Zrinj Gebirge $615 \mathrm{~m}$.

\begin{tabular}{|c|c|c|c|c|c|c|c|c|c|c|c|}
\hline \multirow{2}{*}{\multicolumn{2}{|c|}{ 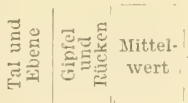 }} & \multicolumn{9}{|c|}{ Berglehnen nach ihrer Exposition } & \multirow{2}{*}{$\begin{array}{l}\text { In } \\
\text { Nllge- } \\
\text { meinen }\end{array}$} \\
\hline & & $\mathrm{N}$ & $\mathrm{NO}$ & 0 & SO & $\mathrm{S}$ & & IV & NIV & $\begin{array}{c}\text { Nittel- } \\
\text { wert }\end{array}$ & \\
\hline
\end{tabular}

\section{Picea excelsa Ik.}

a) vereinzelt, untere Grenze (in Allgemeinen)

\begin{tabular}{|c|c|c|c|c|c|c|c|c|c|c|}
\hline Summe & & 803 & 2657 & 1322 & 1660 & 290 & 551 & 690 & 621 & 8591 \\
\hline Anzahl & $\stackrel{5}{0}$ & 2 & 5 & 3 & 2 & 1 & 1 & 2 & 2 & 18 \\
\hline Mittel & $\Rightarrow$ & 401 & 531 & 441 & 830 & 290 & 551 & 345 & 310 & 477 \\
\hline Min. & 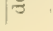 & & & 159 & & & & & & \\
\hline
\end{tabular}

b) rein oder vorherrschend, untere Grenze (im Allgemeinen)

\begin{tabular}{|c|c|c|c|c|c|c|c|c|c|}
\hline Summe & \multirow{4}{*}{ 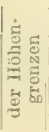 } & 638 & 3372 & 1105 & 1679 & 624 & 1296 & 1391 & 10105 \\
\hline Anzahl & & 1 & 5 & 2 & 3 & 1 & 2 & 2 & 16 \\
\hline Mittel & & 638 & $67 \pm$ & 552 & 560 & 624 & 648 & 695 & 632 \\
\hline Min. & & & & & $35 \tilde{\jmath}$ & & & & \\
\hline
\end{tabular}

c) vereinzelt, untere Grenze an der Landseite

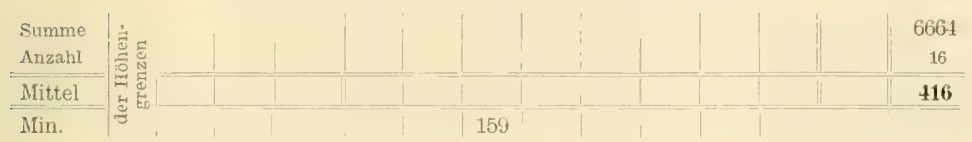

d) rein oder rorhersschend, untere Grenze an der Landseite

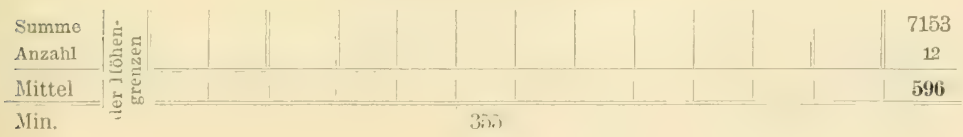




焉

e) untere Grenze an der Meerseite

\begin{tabular}{l|l|c|c|c|c|c|c||c} 
Summo \\
Anzahı \\
Mittel
\end{tabular}

f) geschlossener Bestand, obere Grenze

\begin{tabular}{|c|c|c|c|c|c|c|c|c|c|}
\hline Simme & 1214 & 41048 & 3846 & 12312 & 1316 & $2 \times 34$ & $13: 1$ & 2835 & 18676 \\
\hline Anzahl & 1 & 3 & 3 & 1 & 1 & 2 & 1 & 2 & 14 \\
\hline Mittel & 1214 & $13 i 3$ & 1282 & $1: 312$ & 1316 & 1417 & $13+1$ & 1417 & 1334 \\
\hline Мax. & & & & & & 1504 & & & \\
\hline
\end{tabular}

g) sporadisches Vorkommen im Buchenbestand, obere Grenze

\begin{tabular}{|c|c|c|c|c|c|c|c|c|}
\hline Summe & 4088 & 4122 & 2877 & 4252 & 2977 & 5756 & 1406 & 257.8 \\
\hline Anzahl & 3 & 3 & 2 & 3 & 2 & 4 & 1 & 18 \\
\hline Mittel & $13 t: 3$ & $1+i t$ & $113 x$ & 1117 & $1+\times 0$ & 1439 & 1446 & 1432 \\
\hline Max. & 1617 & & & & & & & \\
\hline
\end{tabular}

h) Raumbestand, obere Grenze

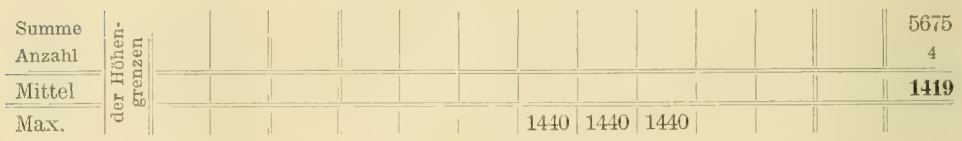

i) Baumgrenze (Bäume mit $8 \mathrm{~m}$. Gipfelhöhe)

\begin{tabular}{l|c|c|c|c|c|c} 
Summe & \\
Anzahl \\
\hline Mittel
\end{tabular}

k) verkrïppelt, obere Grenze

\begin{tabular}{|c|c|c|c|c|c|c|c|c|}
\hline simme & 3337 & fit12 & $165 \mathrm{t}$ & 3210 & $1+i 2 x$ & 1620 & 1617 & 19534 \\
\hline L1нzahl & 2 & 4 & 1 & 2 & 1 & 1 & 1 & 12 \\
\hline Nittel & 1665 & 1610 & 1605 & 1630 & $16 i 2 x$ & 1620 & $14 i 17$ & $16 \geq 8$ \\
\hline Max. & 1699 & & & & & & & \\
\hline
\end{tabular}




Perglehmen nach ihrer Exposition
No

\section{Abies pectimata DC. (A. alba Hill.)}

a) untere Grenze (im Altgemeinen)

\begin{tabular}{|c|c|c|c|c|c|c|c|c|c|c|c|c|c|}
\hline Summe & & 3243 & 3243 & 14619 & 12060 & 3862 & 6413 & 3050 & 6737 & 3920 & 4183 & 53874 & 57117 \\
\hline Anzahl & $\frac{0}{5}$ & 7 & 7 & 22 & 19 & 7 & 8 & 4 & 19 & 5 & 7 & 81 & 88 \\
\hline $\begin{array}{l}\text { Mittel } \\
\text { Min. }\end{array}$ & 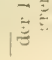 & 4133 & $4+3$ & tifin & $\begin{array}{l}f 3,1 \\
304\end{array}$ & $\pi{ }^{2}$ & (4) & 702 & 719 & N1 & in & (ini & (6)! \\
\hline
\end{tabular}

b) untere Grenze an der Landseite

\begin{tabular}{|c|c|c|c|c|c|c|c|c|c|c|c|c|c|}
\hline Summo & 1. & 3243 & 3243 & 13171 & 12060 & 3862 & 5579 & $|3130|$ & 4019 & 2023 & 4183 & 47027 & 50270 \\
\hline Anzahl & : & 7 & 7 & 20 & 19 & 7 & 7 & 3 & 6 & 4 & 7 & 73 & 80 \\
\hline Mittel & $\Xi 5$ & +133 & 413 & (iji).t & (13) & $5 \pi 2$ & $7 ! \pi$ & 7161 & 6.70 & 506 & $5 \operatorname{ts}$ & $6+1$ & 628 \\
\hline Min. & & & & & 201 & & & & & & & & \\
\hline
\end{tabular}

c) untere Grenze an der Meerscite

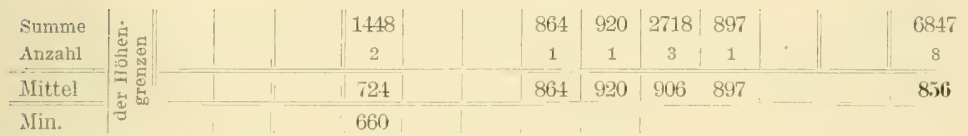

d) rein oder vorherrschend, obere Grenze

\begin{tabular}{|c|c|c|c|c|c|c|c|c|c|c|}
\hline Summe & \multirow{4}{*}{ 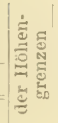 } & 2378 & 5856 & 47.49 & 2337 & 1142 & 5947 & 1381 & 2761 & 26551 \\
\hline Anzalil & & 2 & 5 & 4 & 2 & 1 & 5 & 1 & 2 & 2 \\
\hline Mittel & & 1189 & 1171 & 1187 & 1169 & 1142 & 1189 & 1381 & 1380 & $120 \overline{7}$ \\
\hline Iax. & & & & & & & & & 1406 & \\
\hline
\end{tabular}

e) vereinzelt (Baumform), obere Grenze

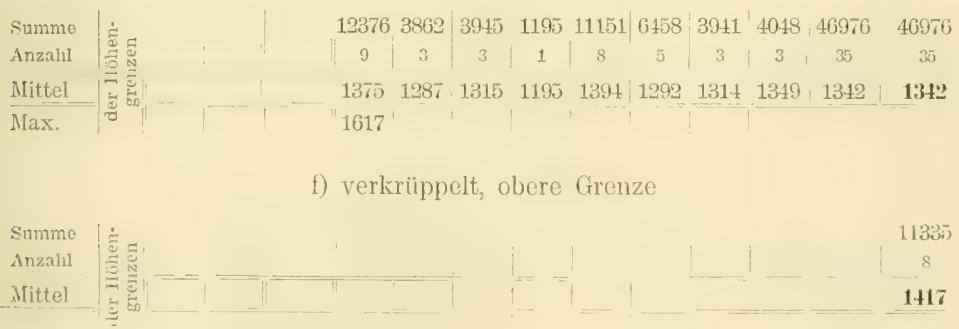




\begin{tabular}{|c|c|c|c|c|c|c|c|c|c|c|c|c|c|c|c|c|}
\hline \multirow{2}{*}{ 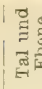 } & & \multirow{2}{*}{\multicolumn{3}{|c|}{ Mittel. }} & \multicolumn{11}{|c|}{ Berglehnen nach ihrer Exposition } & \multirow{2}{*}{$\begin{array}{c}\text { Im } \\
\text { Allge- } \\
\text { meinen }\end{array}$} \\
\hline & & & & & & & NO & $c$ & & 0 & S & SW & $\mathrm{W}$ & NH & $\begin{array}{c}\text { Mittel- } \\
\text { wert }\end{array}$ & \\
\hline
\end{tabular}

Pinus silvestris L.

a) vereinzelt, untere Grenze

\begin{tabular}{|c|c|c|c|c|c|c|c|c|c|c|}
\hline $\begin{array}{l}\text { Summe } \\
\text { Anzahl }\end{array}$ & $\begin{array}{c}168 \\
1\end{array}$ & $\begin{array}{c}168 \\
1\end{array}$ & $\begin{array}{c}685 \\
3\end{array}$ & $\begin{array}{c}441 \\
2\end{array}$ & $\begin{array}{c}840 \\
2\end{array}$ & $\begin{array}{c}861 \\
2\end{array}$ & $\begin{array}{c}490 \\
1\end{array}$ & $\begin{array}{c}222 \\
1\end{array}$ & $\begin{array}{c}3539 \\
11\end{array}$ & $\begin{array}{c}3707 \\
12\end{array}$ \\
\hline Mittel & 168 & 168 & 228 & 220 & 430 & 431 & 490 & 22 & 320 & 309 \\
\hline Min. & & & & 164 & & & & & & \\
\hline
\end{tabular}

b) bestandbildend, untere Grenze

\begin{tabular}{l|c||c|c|c|c|c|c|c|c}
$\begin{array}{l}\text { Summe } \\
\text { Anzahl }\end{array}$ \\
\hline $\begin{array}{l}\text { Mittel } \\
\text { Min. }\end{array}$
\end{tabular}

c) obere Grenze

\begin{tabular}{l|l|l|c|c|c|c||c} 
Summe \\
Anzahl \\
\hline Mittel
\end{tabular}

Pinus nigra Aru.

a) vereinzelt, untere Grenze

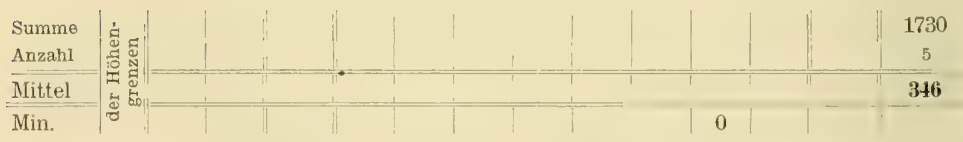

b) bestandbildend, untere Grenze

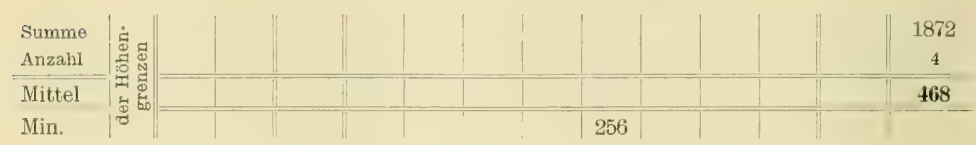

c) bestandbildend, obere Grenze

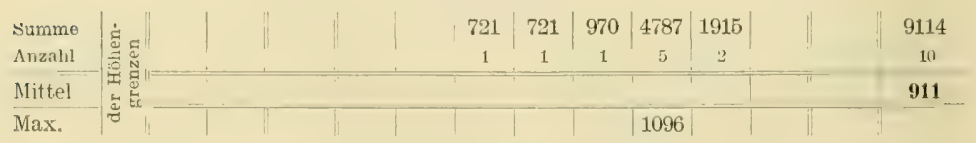




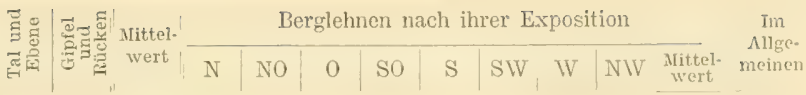

d) vereinzelt (Baumform), obere Grenze

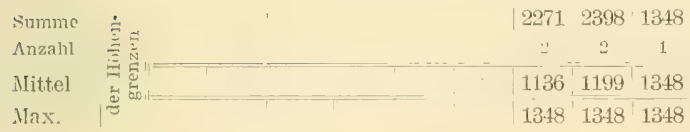

\section{Pinus montana Mill.}

untere Grenze

\begin{tabular}{|c|c|c|c|c|c|c|c|c|c|}
\hline Sinmme & & 11029 & 2500 & 5127 & 1441 & 5693 & 4378 & 1412 & 31616 \\
\hline Inzahl & 苛 & 8 & 2 & 4 & 1 & 4 & 3 & 1 & 23 \\
\hline Mittel & $\vec{z}$ & 1379 & $125 \underline{3}$ & 1282 & $14 \pm 1$ & 1423 & 1459 & 1442 & 1375 \\
\hline
\end{tabular}

'Taxus baceata L.

a) untere Grenzo

\begin{tabular}{|c|c|c|c|c|}
\hline Summe & 1851 & 396 & $6+3$ & 2890 \\
\hline Anzahl & 4 & 1 & 1 & 6 \\
\hline Mittel & 463 & 396 & 643 & 182 \\
\hline Min. & 261 & & & \\
\hline
\end{tabular}

b) obere Grenze

\begin{tabular}{|c|c|c|c|c|c|c|c|c|c|}
\hline Summe & $\therefore \quad 1180$ & 4254 & 2375 & 3250 & 2060 & 2317 & 1000 & 3564 & 20000 \\
\hline Anzain & 足 & 4 & 2 & 3 & 2 & 2 & 1 & 3 & 18 \\
\hline Mittel & 1180 & 1064 & 1187 & 1083 & 1030 & 1168 & 1000 & 1188 & 1111 \\
\hline Max. & $=1$ & & 1385 & & & & & 1385 & \\
\hline
\end{tabular}

\section{Juniperus communis L.}

a) untere Grenze an der Meerseite

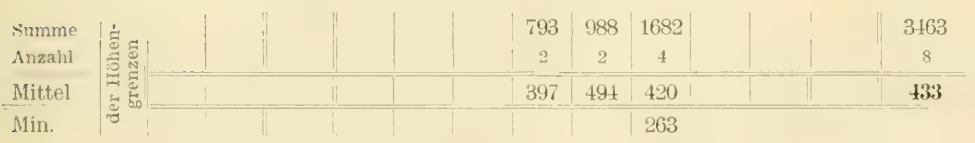




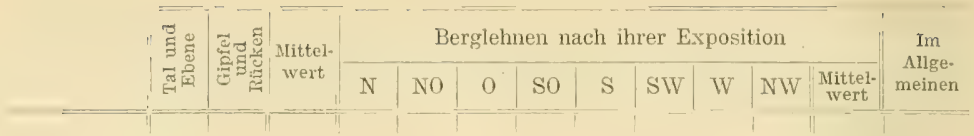

b) obere Grenze

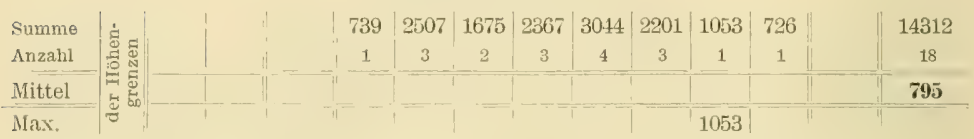

\section{Juniperus nana Willd.}

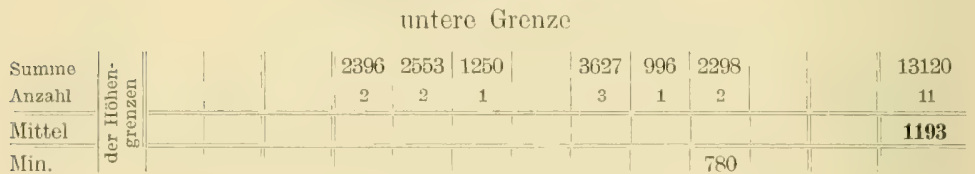

Jumiperus Oxycedrus $\mathbf{I}$.

obere Grenze

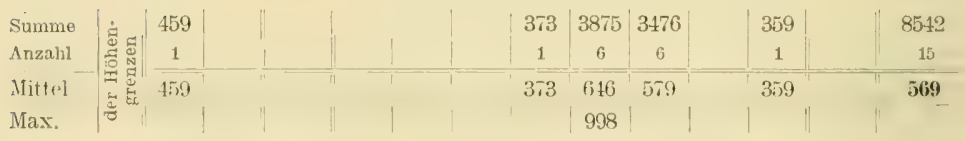

Juniperus Sabiua 1.

untere Grenze

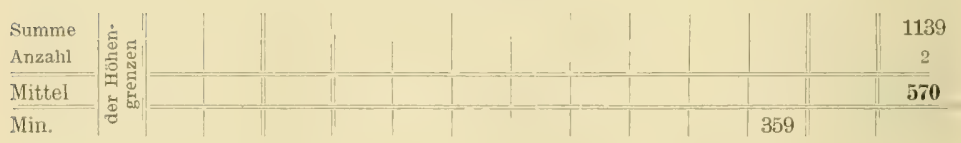

Fagus silvatica $\mathbf{L}$.

a) vereinzelt, untere Grenze (im Allgemeinen)

\begin{tabular}{|c|c|c|c|c|c|c|c|c|c|c|c|c|c|}
\hline Summe & & 2951 & 2951 & 5402 & 5953 & 1703 & 18 ปี & 4997 & 6090 & 3798 & 2849 & 32649 & 35600 \\
\hline Anzah & & 9 & 9 & 20 & 21 & 8 & 5 & 9 & 12 & 11 & 9 & 95 & 104 \\
\hline $\begin{array}{l}\text { litte. } \\
\text { lin. }\end{array}$ & . & $\begin{array}{c}328 \\
97\end{array}$ & 338 & 370 & 283 & 213 & 372 & 5505 & 5018 & 345 & 317 & 341 & 342 \\
\hline
\end{tabular}




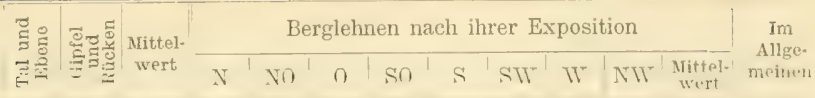

b) rein oder vorherrschend, untere Grenze (im Allgemeinen)

\begin{tabular}{|c|c|c|c|c|c|c|c|c|c|c|c|c|}
\hline S"มmนาก & 1913 & 1912 & $152+15$ & $1 \% 19$ & $i 314$ & 5itii & 10,st; & 8625 & $136 \mathrm{~s}$ & $5: 212$ & $15660 \%$ & 6 \\
\hline Anzalil & 5 & 5 & 3 & 3 & 1.7 & [11 & if & 11 & 5 & 12 & 134 & 4 \\
\hline Tittel & $3 \times 2$ & 383 & 137 & 5,2 & 3.1 & ताT & bis. & ist & 274 & tiit & 490 & \\
\hline Min. & & & & 123 & & & & & & & & \\
\hline
\end{tabular}

c) vereinzelt, untere (irenze an der Meerseite

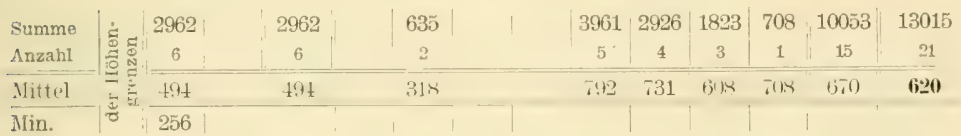

d) rein oder vorherrschend, untere Grenze an der Meerseite

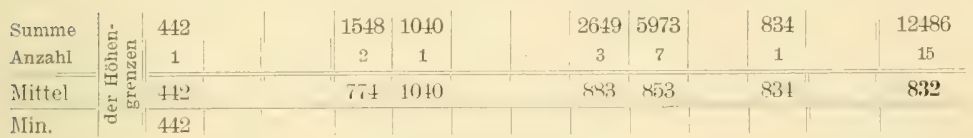

e) vereinzelt, untere Grenze an den kroatischen Hochebenen

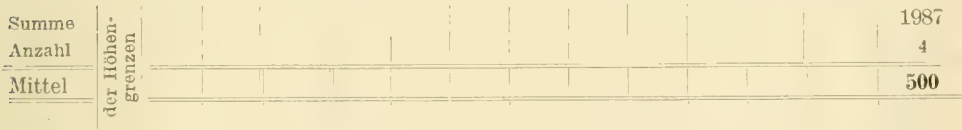

f) rein oder vorherrschend, untere Grenze an den kroatischen Hochebenen

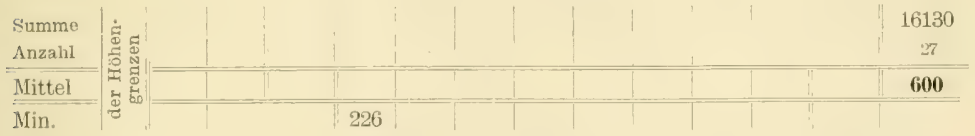

g) untere Grenze des sporadischen Torkommens im Becken der Dran, Situ und Kulpa

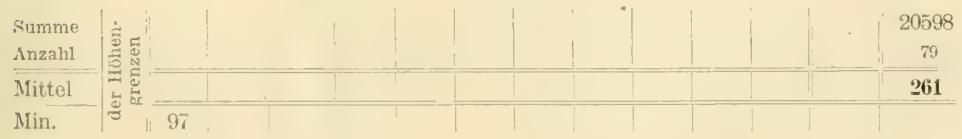




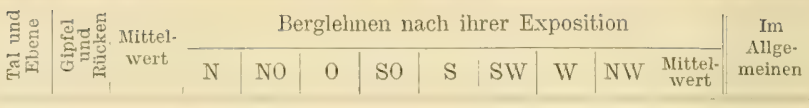

h) rein oder vorherschend, untere Grenze in Becken der Drau, Save und Kulpa

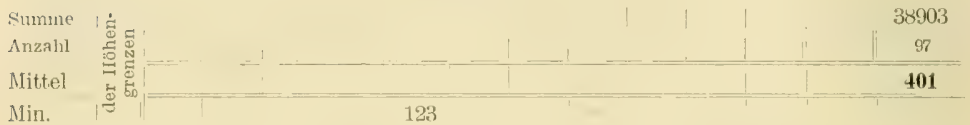

i) Bestandbildung, obere Grenze

\begin{tabular}{|c|c|c|c|c|c|c|c|c|c|c|c|c|c|}
\hline Strmme & & 12410 & $12+40$ & 19275 & 18012 & $390 b$ & 6679 & 13608 & 5698 & 5179 & 7991 & $803+8$ & 92793 \\
\hline Anzalil & 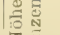 & . 9 & 9 & 14 & 13 & 3 & 5 & 10 & 4 & 4 & 6 & 59 & 68 \\
\hline Mittel & $\div 8$ & $13 \times 3$ & $13 \times 3$ & 1375 & $13 x t$ & 1302 & $133 t j$ & $13+i 1$ & $1.1: 1$ & $13 x$ & 1332 & 1303 & 136i) \\
\hline Max. & | & & & $161 \%$ & & & & & & & & & \\
\hline
\end{tabular}

k) vereinzelt, obere Grenze in lichten- und Tamenbeständen

\begin{tabular}{|c|c|c|c|c|c|}
\hline Summe & & 1182 & 3003 & 1381 & 5566 \\
\hline Anzalul & 5 & 1 & 2 & 1 & 4 \\
\hline Mittel & 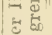 & & & & 1391 \\
\hline
\end{tabular}

1) Strauchform (verkriippelte Bestände), obere Grenze

\begin{tabular}{|c|c|c|c|c|c|c|c|c|c|c|c|c|c|}
\hline Summe & & 5741 & $57 \pm 1$ & 10839 & 6140 & 7504 & 1428 & 12170 & 3028 & 1630 & 7456 & 50195 & รัธั936 \\
\hline Anzahl & 욤 & 4 & 4 & 7 & 4 & 5 & 1 & 8 & 2 & 1 & 5 & 33 & 37 \\
\hline Mittel & & 1 tije) & $1+3+$ & Jistis & 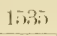 & $1 ; x) 1$ & 1428 & 1521 & 151.4 & 1630 & 1491 & 1521 & 1512 \\
\hline Max. & & & & 1699 & & & & & & & & & \\
\hline
\end{tabular}

\section{Querens sessiliflora Salisb.}

a) intere Grenze an der Meerseite

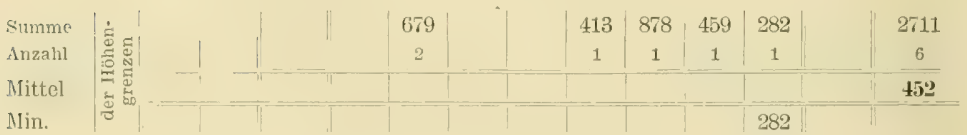




\begin{tabular}{|c|c|c|c|c|c|c|c|c|c|c|c|}
\hline \multirow{2}{*}{ 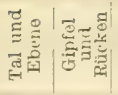 } & \multirow{2}{*}{$\begin{array}{l}\text { Mittel- } \\
\text { wert }\end{array}$} & \multicolumn{9}{|c|}{ Berglehnen nach ihrer Exposition } & \multirow{2}{*}{$\begin{array}{c}\text { Im } \\
\text { Allye- } \\
\text { meinen }\end{array}$} \\
\hline & & N & No & 0 & So & $\mathrm{S}$ & SW & W & NW & $\begin{array}{l}\text { Mittel- } \\
\text { wert }\end{array}$ & \\
\hline
\end{tabular}

b) rein oder vorherrschend, obere Grenze

\begin{tabular}{l|c|c|c|c|c|c|c|c|c|c} 
Summe \\
Anzahl \\
\hline Mittel
\end{tabular}

c) vereinzelt, obere Grenze

\begin{tabular}{|c|c|c|c|c|c|c|c|c|}
\hline Summe & 696 & 1016 & 1828 & 1127 & 1475 & | 6788 | & 5112 & 18042 \\
\hline Anzahl & 1 & 1 & 3 & 2 & 2 & 8 & 6 & 23 \\
\hline Mittel & 696 & 1016 & 609 & $5 f) \pm$ & 738 & $81 x$ & $8 \pi 2$ & 785 \\
\hline Max. & & & & & & & 1044 & \\
\hline
\end{tabular}

\section{Quereus peduneulata Ehrh. (Qu. Robur L.)}

a) untere Grenze an der Meerseite

Min. \| $73 \mid$

b) obere Grenze

\begin{tabular}{|c|c|c|c|c|c|c|c|c|c|}
\hline Summe & 4113 & 1036 & 764 & 1135 & 571 & 1372 & 1211 & 534 & 10736 \\
\hline Anzahl & 7 & 2 & 2 & 2 & 1 & 2 & 2 & 1 & 19 \\
\hline Mittel & 588 & ๘18 & 382 & 568 & 571 & $680^{\circ}$ & 600 & $53 t$ & 565 \\
\hline Max. & 709 & & & & & & & & \\
\hline
\end{tabular}

\section{Quercus lanuginosa Lam.}

a) bestandbildend, obere Grenze

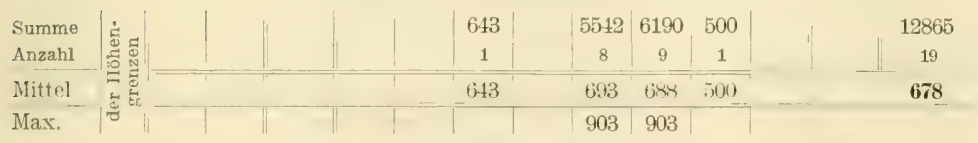

b) vereinzelt (Baumform), obere Grenze

\begin{tabular}{|c|c|c|c|c|c|c|c|}
\hline Summe & & 1249 & 2212 & 11175 & 3976 & 1635 & 20247 \\
\hline Anzahl & : & 2 & 3 & 14 & 5 & 2 & 26 \\
\hline Nittel & 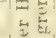 & 625 & 737 & 7.18 & 795 & $81 \overline{7}$ & 789 \\
\hline Max. & $\left.\right|^{2}$ & & & & & 1084 & \\
\hline
\end{tabular}




Berglehnen nacls ihrer Exposition Im Im
IItw..

c) verkrüppelt, obere Grenze

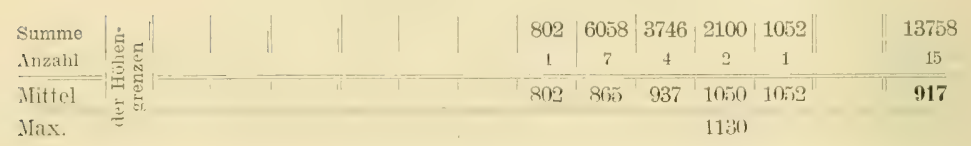

\section{Quereus Hex L.}

obere Grenze

$\operatorname{Max}|\||\||+|| 161$

\section{Quercus Cerris L.}

a) untere Grenze an der Meerseite

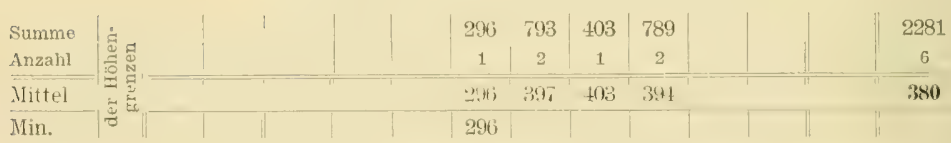

b) bestandbildend, obere Grenze

\begin{tabular}{|c|c|c|c|c|c|c|c|c|c|}
\hline Summe & & 747 & 660 & 1374 & 1330 & 7994 & 4536 & 3764 & 20405 \\
\hline Anzaht & & 1 & 1 & 2 & $\ddot{z}$ & 11 & ii & 5 & 28 \\
\hline Mittel & 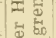 & 747 & 660 & 687 & 665 & 727 & 756 & 753 & 729 \\
\hline Max. & & & & & & 962 & & & \\
\hline
\end{tabular}

c) vereinzelt (Baumform), obere Grenze

\begin{tabular}{|c|c|c|c|c|c|c|c|c|c|}
\hline Summe & & 7.1 & 1765 & 2323 & 931 & 3414 & 3963 & 809 & 13979 \\
\hline Anzahi & & 1 & 2 & 3 & 1 & 4 & 5 & 1 & 17 \\
\hline Mittel & जि & 771 & 882 & 774 & 934 & 854 & 793 & 809 & 822 \\
\hline Max. & & & & & & 987 & 987 & & \\
\hline
\end{tabular}

d) verkriippelt, obere Grenze

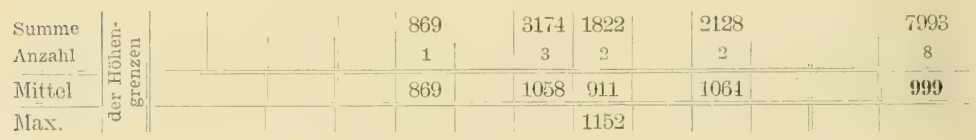




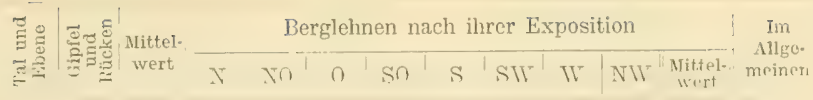

\section{Castanea sativa Mill.}

a) vereinzelt, untere Grenze

\begin{tabular}{|c|c|c|c|c|c|c|c|c|c|c|}
\hline Summe & & 1812 & 1338 & 344 & 752 & 897 & 713 & 332 & 385 & 6573 \\
\hline AnzahI & 롬 & ]1 10 & 6 & 2 & 3 & 5 & 4 & 2 & 2 & 34 \\
\hline Mittel & 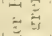 & $1 \$ 1$ & $3-23$ & 172 & $\therefore \% 1$ & 1.9 & $17 \pi$ & $16 t ;$ & 192 & 193 \\
\hline Min. & $\mid ت$ & & 123 & & & & & & & \\
\hline
\end{tabular}

b) bestandbildend, untere Grenze

\begin{tabular}{|c|c|c|c|c|c|c|c|c|}
\hline Summe & \multirow{4}{*}{ 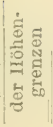 } & 376 & 197 & 535 & 439 & 778 & 599 & 2851 \\
\hline Anzahl & & 2 & 1 & 2 & 2 & 3 & 2 & 12 \\
\hline Mittel & & 188 & 197 & 268 & 219 & 259 & 265 & 238 \\
\hline Min. & & 169 & & & & & & \\
\hline
\end{tabular}

c) bestandbildend, obere Grenze

\begin{tabular}{|c|c|c|c|c|c|c|c|c|c|}
\hline Summe & $\dot{\vec{E}}$ & 1066 & 1695 & 371 & 1042 & 552 & 1672 & 581 & 6979 \\
\hline Anzahl & $\because \frac{1}{8}$ & 2 & 3 & 1 & 12 & 1 & 3 & 1 & 13 \\
\hline Mittel & $=5$ & 533 & (ifi.) & 3.1 & 521 & ind & $i n \pi$ & $5 \times 1$ & .3 .37 \\
\hline Max. & $1 \frac{3}{0}$ & 600 & 600 & & & & 600 & & \\
\hline
\end{tabular}

d) vereinzelt, obere Grenze

\begin{tabular}{|c|c|c|c|c|c|c|c|c|c|}
\hline Summo & & 560 & 1122 & 476 & 367 & 1216 & 559 & 1970 & 6270 \\
\hline Anzahl & $\frac{0}{5}$ & 1 & 2 & 1 & 1 & 2 & 1 & ; 3 & 11 \\
\hline Xittr.1 & $=\sum_{0=1}$ & if in & intil & titi & 337 & (ivis & ก154 & fini 7 & 570 \\
\hline Max. & 1 & & & & & 740 & & & \\
\hline
\end{tabular}

\section{Juglans regia L.}

obere Grenze

\begin{tabular}{|c|c|c|c|c|c|c|c|c|}
\hline Summe & ¿ 577 & 552 & 1338 & 1102 & 1151 & 386 & 525 & 5631 \\
\hline Anzahl & 䒕 & 1 & 2 & 2 & 2 & 1 & 1 & 10 \\
\hline Mittel & $\Rightarrow$ & $\therefore$ & & & & & & 563 \\
\hline Miax. & $\approx$ & & 786 & & & & & \\
\hline
\end{tabular}




\begin{tabular}{|c|c|c|c|c|c|c|c|c|c|c|c|c|}
\hline \multirow{2}{*}{ 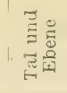 } & \multirow{2}{*}{\multicolumn{2}{|c|}{ 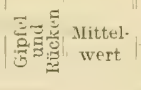 }} & \multicolumn{9}{|c|}{ Berglehnen nach ihrer Exposition } & \multirow{2}{*}{$\begin{array}{l}\text { Im } \\
\text { Allge- } \\
\text { meinen }\end{array}$} \\
\hline & & & 1 & NO & 0 & SO & S & SIV & W & NW & $\begin{array}{c}\text { Mittel- } \\
\text { wert }\end{array}$ & \\
\hline
\end{tabular}

\section{Carpinus Betulus $\mathbf{L}$.}

a) untere Grenze an der Meerseite

Min. $349^{\circ}$

b) bestandbildend, obere Grenze

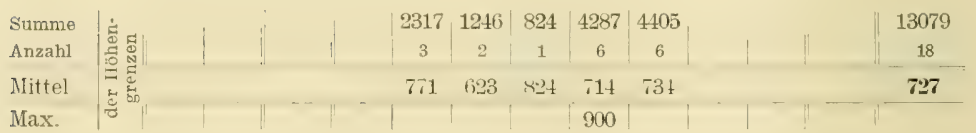

c) vereinzelt (Baumform), obere Grenze

\begin{tabular}{|c|c|c|c|c|c|c|c|c|c|c|c|}
\hline Summe & 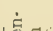 & $2 x(1) 9$ & 2391 & 759 & 3174 & $301 \pm$ & 3817 & 2499 & 703 & 18995 & 18995 \\
\hline Anzahl & : & 4 & 3 & 1 & 4 & 4 & 5 & 3 & 1 & 25 & 25 \\
\hline Mitt.el & $=5$ & 702 & 740 & 759 & 794 & $75 t$ & 763 & 833 & 703 & 760 & 760 \\
\hline Max. & เซ & & & & & & & 1035 & & & \\
\hline
\end{tabular}

d) Strauchform, obere Grenze

\begin{tabular}{l|l|l|l|l|l|l||} 
Summe \\
Anzahl \\
\hline Mittel
\end{tabular}

Carpinus duinensis Seop.

obere Grenze

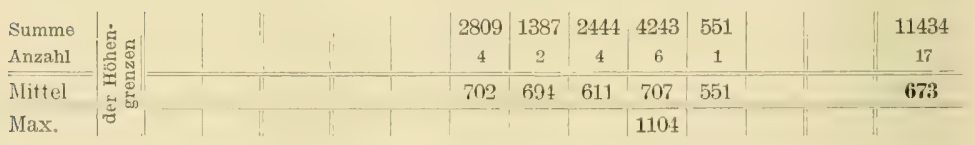

Ostrya carpinifolia Scop.

a) Baumform, obere Grenze

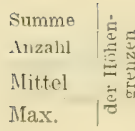

\begin{tabular}{|c|c|c|c|c|c|c|c|c|}
\hline 787 & 1905 & 2834 & 6482 & 11128 & 8314 & 3416 & 745 & 35611 \\
\hline 1 & 3 & 3 & 8 & 13 & 111 & 4 & 1 & 43 \\
\hline 787 & $63 i$ & 945 & 810 & $85 \tilde{6}$ & 831 & 854 & 745 & 828 \\
\hline
\end{tabular}




\begin{tabular}{|c|c|c|c|c|c|c|c|c|c|c|c|}
\hline \multirow{2}{*}{ 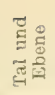 } & \multirow{2}{*}{ 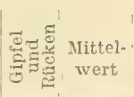 } & \multicolumn{9}{|c|}{ Berglehnen nach ilurer Exposition } & \multirow{2}{*}{$\begin{array}{l}\text { Im } \\
\text { Allge- } \\
\text { meiner }\end{array}$} \\
\hline & & $N$ & No & 0 & & $\mathrm{~S}$ & SW & W & NW : & $\begin{array}{c}\text { Mittel- } \\
\text { wert }\end{array}$ & \\
\hline
\end{tabular}

b) Strauchform, obere Grenze

\begin{tabular}{l|c|c|c|c|c|c|c|c|c|c|c|} 
Summe & & 1130 & 611 & 895 & 953 & 4969 & 9318 & 12977 & 5323 & 2616 & 38792 \\
Anzahl
\end{tabular}

\section{Corylus Arellana L.}

a) untere Grenze an der Meerseite

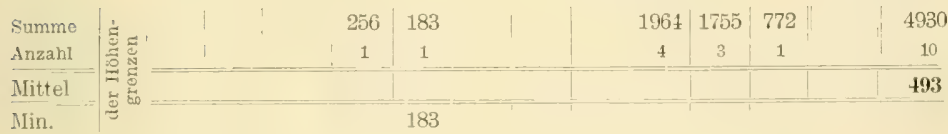

b) obere Grenze

\begin{tabular}{|c|c|c|c|c|c|c|c|c|c|c|c|c|c|}
\hline Summe & & 4310 & 4310 & 3245 & 3594 & 2754 & 6195 & 7589 & 6243 & 7833 & 1010 & 38463 & 42773 \\
\hline Anzahl & 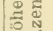 & 4 & 4 & 3 & 4 & 3 & 6 & 7 & 6 & 8 & 1 & 38 & 42 \\
\hline Mittel & $=5$ & $10 \pi x$ & $10 . \bar{x}$ & 10R2 & 899 & $91 \overline{8}$ & 1032 & 1091 & 1010 & $9-9$ & 1010 & 1012 & 1018 \\
\hline Iax. & & & & & & & & 1481 & & & & & \\
\hline
\end{tabular}

\section{Betula verrucosa Elirh.}

obere Grenze

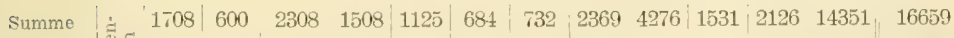

\begin{tabular}{|c|c|c|c|c|c|c|c|c|c|c|c|c|c|c|}
\hline Anzahl & $\frac{2}{5}$ & 3 & 1 & 4 & 2 & 2 & 1 & 1 & 3 & 6 & 2 & 3 & 20 & 24 \\
\hline Mittel & 5 & โร9 & 601 & 57 & int & iff? & fint & 732 & $T ! H !$ & 713 & ititi & 7169 & 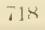 & 694 \\
\hline
\end{tabular}

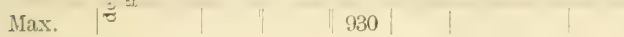

\section{Aluus glutinosa Girtn.}

obere Grenze

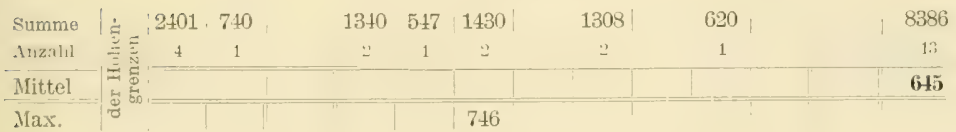




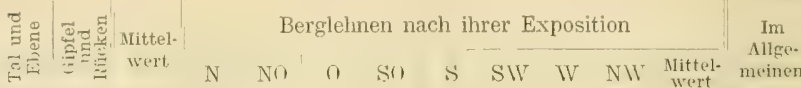

Aluns incana Willd.

a) mitere Grenze an der Neerseite

Min.

349

b) obere Grenze

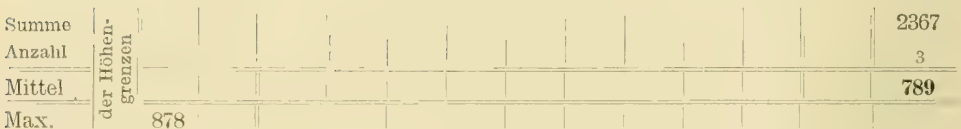

Salix alba L.

obere Grenze

\begin{tabular}{|c|c|c|c|c|c|c|}
\hline Summe & 5791 & 648 & 751 & 518 & 717 & 8155 \\
\hline Irzahl & $\frac{1}{3} \bar{\Xi}$ & 1 & 1 & 1 & 1 & 12 \\
\hline Mittel & 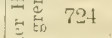 & bitk & (i) 1 & its & 717 & 70.5 \\
\hline Max. & " & & & & & \\
\hline
\end{tabular}

Populus tremula I.

a) Baumform, obere Grenze

\begin{tabular}{|c|c|c|c|c|c|c|c|c|c|c|}
\hline Summe & & 1584 & 4282 & 3807 & 3629 & 2621 & 860 & 1700 & 683 & 19166 \\
\hline Anzahl & : & 2 & 5 & 4 & 4 & 3 & 1 & 2 & 1 & 2 \\
\hline Mitt.u & & 70 & sitti & $(4) 2$ & $90-i$ & sit & $\mathrm{A}(i)$ & 850 & lik3 & 871 \\
\hline Iax. & & & & & 1151 & & & & & \\
\hline
\end{tabular}

b) verkrüppelt, obere Grenze

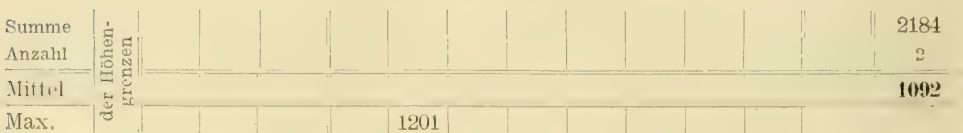




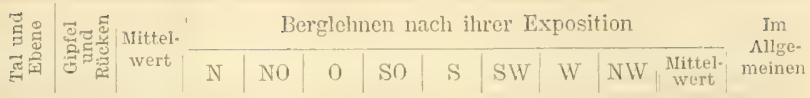

Ulmus montama With. (U. seabra Mill.)

a) untere Grenzo

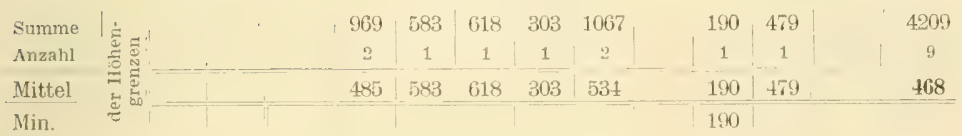

b) Baumform, obere Grenze

\begin{tabular}{|c|c|c|c|c|c|c|c|c|c|c|}
\hline sinmme & $1 \dot{\approx}$ & 2545 & 5034 & 1085 & 1915 & 2679 & 4171 & 1047 & 1830 & 20256 \\
\hline Anzalıl & 气 & 2 & 5 & 1 & 2 & 2 & 4 & 1 & 2 & 19 \\
\hline Mittel & $\begin{array}{ll}F & \vec{\pi} \\
2 & \sum_{0}\end{array}$ & $12-2$ & 1007 & 1035 & 958 & $13 \pm 0$ & $10+3$ & 1047 & 915 & 1066 \\
\hline Max. & & & & & & & 1350 & & & \\
\hline
\end{tabular}

c) Stranchform, obere Grenze

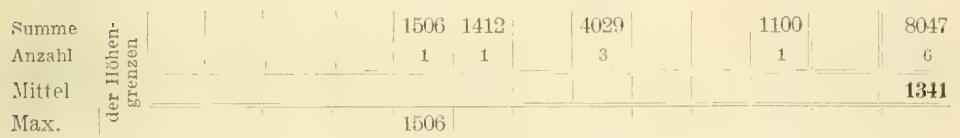

'Tilia parvifolia Ehrh. (T'. cordata Mill.)

a) untere Grenze an der Meerseite

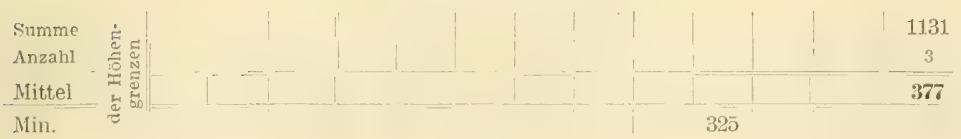

b) obere Grenze

\begin{tabular}{|c|c|c|c|c|c|c|c|c|}
\hline Summe & 1130 & 3251 & 906 & 6670 & | 1214 & 1750 & 1020 & 15941 \\
\hline Anzahl & 11 & 3 & 1 & 6) & 1 & 2 & 1 & 15 \\
\hline Mittel & 1130 & 1084 & 906 & 1112 & $1214^{\circ}$ & 875 & 1020 & 1063 \\
\hline Max. & & & & 1535 & & & & \\
\hline
\end{tabular}




\begin{tabular}{|c|c|c|c|c|c|c|c|c|c|c|c|c|}
\hline \multirow{2}{*}{ 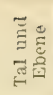 } & \multirow{2}{*}{ 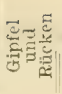 } & \multirow{2}{*}{$\begin{array}{l}\text { Mittel- } \\
\text { wert }\end{array}$} & \multicolumn{9}{|c|}{ Berglehnen nach ihrer Exposition } & \multirow{2}{*}{$\begin{array}{c}\operatorname{Im} \\
\text { Allge- } \\
\text { neines }\end{array}$} \\
\hline & & & $\mathrm{N}$ & NO & 0 & SO & $\mathrm{S}$ & $\mathrm{SW}$ & $\mathrm{W}$ & $\mathrm{NWV}$ & $\begin{array}{c}\text { Mittel- } \\
\text { wert }\end{array}$ & \\
\hline
\end{tabular}

\section{Icer Pseudoplatanus L.}

\begin{tabular}{|c|c|c|c|c|c|c|c|c|c|c|c|}
\hline Summe & & 190 & 1954 & 738 & 710 & 2210 & 1207 & 3363 & 963 & 1006 & 12341 \\
\hline Anzahl & 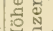 & 1 & 6 & 3 & 4 & 4 & 3 & 4 & 3 & 4 & 32 \\
\hline Mittel & $=5$ & 190 & 326 & $2+6$ & $17 x$ & 552 & 408 & $x+1$ & 321 & 250 & 886 \\
\hline Min. & 10 & & & & 120 & & & & & & \\
\hline
\end{tabular}

b) untere Grenze an der Meerseite

\begin{tabular}{|c|c|c|c|c|c|c|c|}
\hline Summe & & 570 & 1766 & 699 & 3363 & 551 & 6949 \\
\hline Anzahl & : & 1 & 2 & 1 & 4 & 1 & 9 \\
\hline Mittel & $E$ & 570 & $8 \times 3$ & 699 & 841 & 551 & 772 \\
\hline Min. & & & & & & 551 & \\
\hline
\end{tabular}

c) untere Grenze an der Landseite

\begin{tabular}{|c|c|c|c|c|c|c|c|c|c|c|}
\hline Summe & & 190 & 1384 & 738 & 710 & 414 & 508 & 412 & 1006 & 5392 \\
\hline Anzahl & 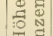 & 1 & 5 & 3 & 4 & 2 & 2 & 2 & 4 & 23 \\
\hline Mittel & $\begin{array}{ll}= \\
5 \\
5\end{array}$ & 190 & $37 \pi$ & 246 & $17 x$ & $23: 2$ & 254 & 3116 & 252 & 334 \\
\hline Min. & 1" & & & & 120 & & & & & \\
\hline
\end{tabular}

d) Baumform, obere Grenze

\begin{tabular}{|c|c|c|c|c|c|c|c|c|c|c|c|c|c|c|}
\hline Summe & & 3630 & 2633 & 5283 & 7787 & $6 \div 01$ & 16.71 & 3011 & 5) 266 & 5313 & 2331 & 3664 & 38682 & 43965 \\
\hline AnzahI & $\mid \begin{array}{l}0 \\
0 \\
0 \\
0\end{array}$ & 2 & 2 & 4 & 6 & 5 & 6 & 2 & 4 & 4 & 2 & 2 & $3 x$ & 35 \\
\hline Mittel & 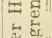 & 1315 & 1326 & 1321 & $12: k$ & 1552 & 1163 & 1029 & 1319 & 1336 & $116 \mathrm{~s}$ & 1339 & 1218 & 1256 \\
\hline Max. & | & & & & & 1507 & & & & & & & & \\
\hline
\end{tabular}

e) Strauchform, obere Grenze

\begin{tabular}{|c|c|c|c|c|c|c|c|c|c|}
\hline Summe & & 4043 & 2983 & 2541 & 3993 & 4023 & 2783 & 1460 & 20826 \\
\hline Anzahl & 롱 & 3 & 2 & 2 & 2 & 3 & 2 & 1 & 15 \\
\hline Mittel & $\Rightarrow z_{0}$ & 13 th & $1+91$ & 1271 & 1497 & $13+1$ & 1392 & 1460 & 1388 \\
\hline Max. & ra & & 1583 & & & & & & \\
\hline
\end{tabular}

\section{Acer platanoides $\mathbf{L}$.}

a) untere Grenze

Min. 


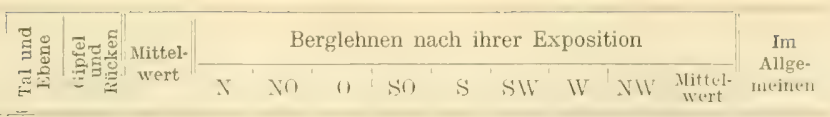

b) Baumform, obere Grenze

\begin{tabular}{|c|c|c|c|c|c|c|c|c|c|c|c|c|}
\hline Summo & & 1009 & 909 & 1918 & 866 & 2999 & 1035 & 1793 & 3655 & 978 & 11326 & 13241 \\
\hline Anzahl & 趈 & 1 & 1 & 2 & 1 & 3 & 1 & 2 & 4 & 1 & 12 & 14 \\
\hline Mittel & $=5$ & 1009 & 909 & $\$ 59$ & Brifi & $10 x x)$ & 10,3 & $x ! \pi$ & 914 & 978 & 914 & 946 \\
\hline
\end{tabular}

c) Stranchform

Max. | 1176

\section{Acer obtusatum W. et K.}

a) Baumform, obere Grenze

\begin{tabular}{|c|c|c|c|c|c|c|c|c|c|c|c|c|c|c|}
\hline Summe & \multirow{4}{*}{ 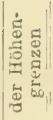 } & 1098 & 2141 & 3239 & 8761 & 5799 & 4779 & 12083 & 15035 & 11793 & 4691 & 1850 & 64791 & 68033 \\
\hline Anzahl & & 1 & 2 & 3. & 11 & 6 & 5 & 14 & 17 & 13 & 5 & 2 & 73 & 76 \\
\hline Mittel & & $10 .+3$ & 1070 & $1081)$ & $7: 46$ & $9+3$ & (5)ti & 813 & $x<t$ & $31 \%$ & 439 & ! & 888 & 895 \\
\hline Max. & & & & & & & & & 1277 & & & & & \\
\hline
\end{tabular}

b) Strauchform, obere Grenze

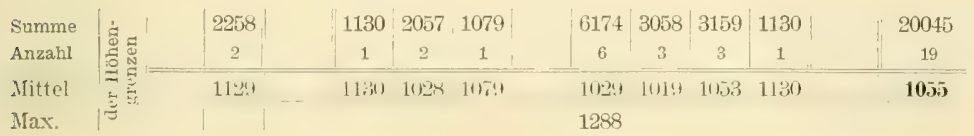

\section{Acer campestre L.}

a) Baumform, obere Grenze

\begin{tabular}{|c|c|c|c|c|c|c|c|c|c|c|}
\hline Summe & & 630 & 1691 & 1463 & 2910 & 5400 & 3865 & 688 & 740 & 17387 \\
\hline Anzahl & $\stackrel{0}{2} \frac{0}{0}$ & 1 & 2 & 2 & 4 & 7 & 5 & 1 & 1 & 23 \\
\hline Mittel & $=$ 至 & 630 & $8+6$ & 732 & 7.98 & 71 & $7 \pi 3$ & 6iRe & 740 & 756 \\
\hline Max. & 10 & & 953 & & & & & & & \\
\hline
\end{tabular}

b) Strauchform, obere Grenze

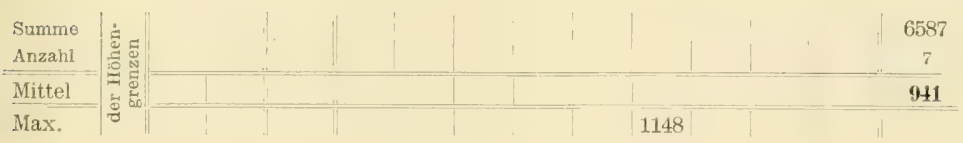




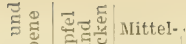

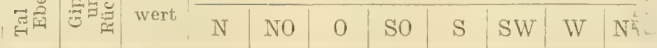

Acer mouspessulanum L.

a) Baumform, obere Grenze

\begin{tabular}{l|l|c|c|c|c|c|c} 
Summe \\
Anzahl \\
\hline Mittel
\end{tabular}

b) Strauchform, obere Grenze

\begin{tabular}{|c|c|c|c|c|c|c|c|c|c|}
\hline Summe & $\dot{\bar{\sigma}}$ & 580 & 1600 & 1679 & 5982 & $40+4$ & 857 & 857 & 15599 \\
\hline Anzahl & & 1 & 2 & 2 & 7 & 6 & 1 & 1 & 20 \\
\hline $\begin{array}{l}\text { Nittel } \\
\text { Max. }\end{array}$ & है & 280 & sitil & $8+1)$ & $\begin{array}{l}\text { sini) } \\
1002\end{array}$ & $67 t$ & 857 & $85 \overline{4}$ & 780 \\
\hline
\end{tabular}

\section{Fraxinus excelsior I.}

a) untere Grenze an der Meerseite

\begin{tabular}{|c|c|c|c|c|c|c|c|}
\hline Summe & $\approx$ & 28 & 451 & 286 & 611 & 810 & 2186 \\
\hline Anzahl & 递 & 1 & 1 & 1 & 1 & 2 & 6 \\
\hline Nittel & $=5$ & & & & & & 364 \\
\hline & & 28 & & & & & \\
\hline
\end{tabular}

b) obere Grenze

\begin{tabular}{|c|c|c|c|c|c|c|c|c|c|}
\hline Summe & $\dot{\Phi} \Xi$ & $\begin{array}{c}950 \\
1\end{array}$ & & $\begin{array}{c}3451 \\
3\end{array}$ & $\begin{array}{c}3198 \\
3\end{array}$ & $\begin{array}{c}2463 \\
2\end{array}$ & $\begin{array}{c}4533 \\
4\end{array}$ & $\begin{array}{c}8135 \\
7\end{array}$ & $\begin{array}{c}27442 \\
24\end{array}$ \\
\hline Mitted & $E$ E & (5)1) & 11 is & 1130 & 10hiti & 1203 & 1133 & 1162 & 1143 \\
\hline tax. & & & & & & & & $|1483|$ & \\
\hline
\end{tabular}

\section{Fraximus Oruus L.}

\begin{tabular}{|c|c|c|c|c|c|c|c|c|c|c|}
\hline Summe & 1130 & 695 & 1908 & 764 & 4551 & |20454| & 9299 & 1551 & 707 & 41059 \\
\hline Anzahl & 1 & 1 & 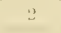 & 1 & 5 & $\ldots$ & 20 & 2 & 1 & 45 \\
\hline Mittal & 1130 & 69.5 & 9.1.1 & 761 & 910 & 930 & 130 & 766 & 707 & 912 \\
\hline & & & & & & 1304 & & & & \\
\hline
\end{tabular}




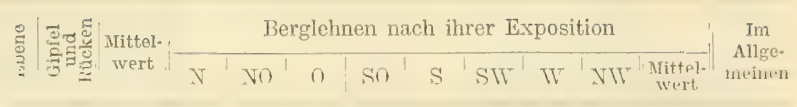

\section{Sorbus aucuparia $\mathbf{L}$.}

a) Baumform, obere Grenze

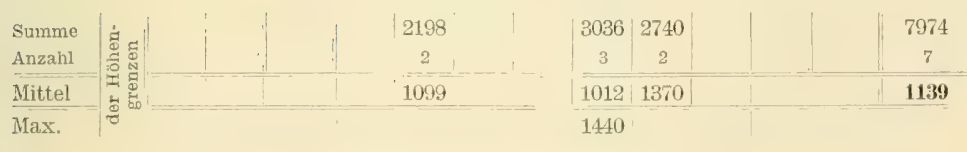

b) Strauchform, obere Grenze

\begin{tabular}{|c|c|c|c|c|c|c|c|c|c|c|}
\hline Summe & $\dot{\varepsilon}$ & 3959 & 5304 & 4240 & 1603 & 1151 & 4571 & 1446 & 1460 & 23728 \\
\hline Anzahl & క్ & 3 & 4 & 3 & 1 & 1 & 3 & 1 & 1 & 17 \\
\hline Mittel & $=5$ & 1320 & 1396 & $1+1 ?$ & 1603 & $11 . \pi 1$ & 1504 & $1 \pm 46$ & $1 \pm 60$ & 1396 \\
\hline Max. & | & & & & & & 1620 & & & \\
\hline
\end{tabular}

\section{Sorbus torminalis Crantz.}

obere Grenze

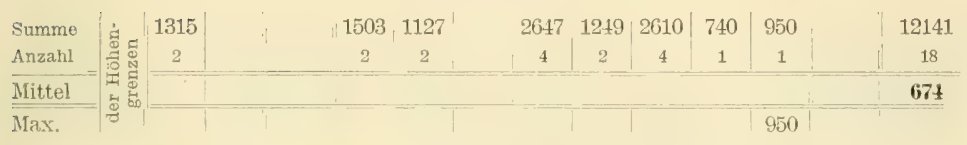

\section{Sorbus Aria Crantz.}

a) untere Grenze

\begin{tabular}{|c|c|c|c|c|c|c|c|c|c|c|}
\hline Summe & & 803 & 195 & 513 & 1766 & 2636 & 3222 & 809 & 466 & 10410 \\
\hline Anzahl & 胥 & 2 & 1 & 2 & 4 & 6 & 7 & 3 & 1 & 26 \\
\hline Mittel & 5 & 401 & 195 & 257 & 442 & 439 & 460 & 2,0 & tijtj & 400 \\
\hline Min. & 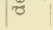 & & & 155 & & & & & & \\
\hline
\end{tabular}

b) Baumform, obere Grenze

\begin{tabular}{|c|c|c|c|c|c|c|c|c|c|}
\hline Summe & & 3909 & 3189 & 2715 & 4682 & 6888 & 1658 & 1890 & 24931 \\
\hline Anzahl & \% & 4 & 3 & 3 & 5 & 7 & 2 & 2 & 26 \\
\hline Mittm & 5 & 9.7 & $10 f 3$ & mii & $(126)$ & $\cos t$ & $\times 39$ & (915) & 954 \\
\hline Max. & & & & & & 1340 & & & \\
\hline
\end{tabular}




\begin{tabular}{|c|c|c|c|c|c|c|c|c|c|c|c|c|}
\hline \multirow{2}{*}{ 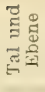 } & \multirow{2}{*}{ 可的 } & \multirow{2}{*}{$\begin{array}{c}\text { Mittel- } \\
\text { wert }\end{array}$} & \multicolumn{9}{|c|}{ Berglehnen nach ihrer Exposition } & \multirow{2}{*}{$\begin{array}{l}\text { Im } \\
\text { Allge. } \\
\text { meinen }\end{array}$} \\
\hline & & & $N$ & NO & 0 & SO & S & SW & $W$ & NWV & $\begin{array}{l}\text { Mittel- } \\
\text { wert }\end{array}$ & \\
\hline
\end{tabular}

c) Strauchform, obere Grenze

\begin{tabular}{|c|c|c|c|c|c|c|c|c|}
\hline Summe & \multirow{4}{*}{ 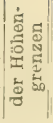 } & 5235 & 2983 & 1095 & 5531 & 3588 & 100094165 & 32606 \\
\hline Anzahl & & 4 & 2 & 1 & 4 & 3 & 8,3 & 25 \\
\hline Mittel & & 1309 & 1491 & 1095 & 1383 & $11 ! 6$ & 1384 & 1304 \\
\hline Max. & & & & & 1603 & & & \\
\hline
\end{tabular}

Crataegus monogyna Jaeq. und oxyacantha $\mathbf{L}$.

\begin{tabular}{l|c|c|c|c|c|c|c|c|c|c|c|c|c|} 
Obere Grenze \\
Summe \\
Anzahl \\
\hline Mittel \\
\hline Max.
\end{tabular}

Prumus Mahaleb L.

obere Grenze

\begin{tabular}{l|c|c|c|c|c|c|c} 
Summe & & & 1750 & 4114 & 3938 & 1104 & 1 \\
Anzahl \\
\hline Mittel
\end{tabular}

\section{Prunus spinosa $\mathbf{L}$.}

a) untere Grenze an der Meerseite

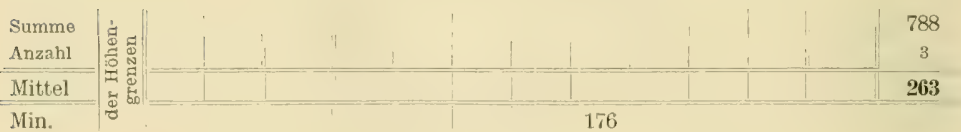

b) obere Grenze

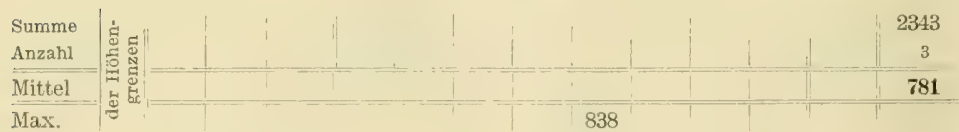




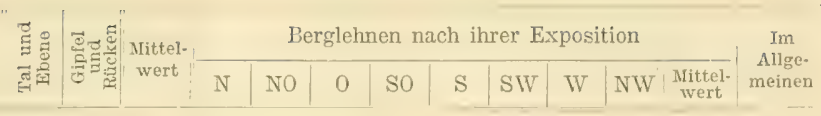

Ficus Carica I.

(verwildert)

obere Grenze

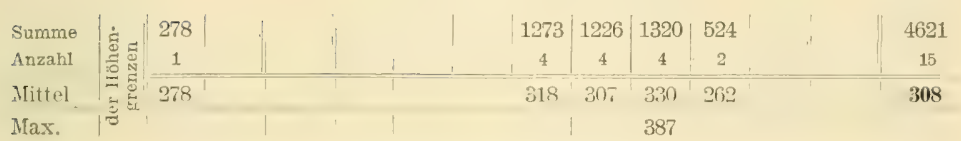

\section{Pistacia 'T'erebinthus L.}

obere Grenze

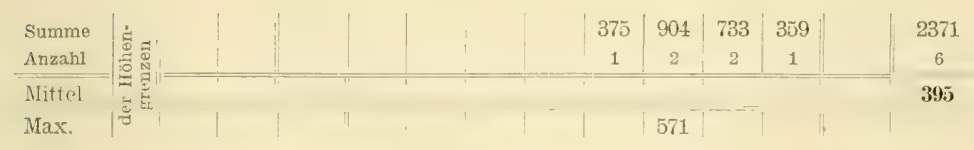

\section{Cotinus Coggygria Scop.}

\begin{tabular}{|c|c|c|c|c|c|c|c|c|c|c|c|}
\hline Summe & & 752 & 752 & 507 & 1431 & 1359 & 4432 & 1468 & 431 & 9628 & 10380 \\
\hline AnzahI & $\mid \frac{1}{0} \frac{a}{0}$ & 1 & 1 & 1 & 2 & 2 & 5 & 2 & 1 & 13 & 14 \\
\hline Mittul & $=5$ & 752 & 752 & 507 & 715 & 680 & 886 & 734 & 431 & $7+1$ & $7+1$ \\
\hline $\operatorname{Max}$. & $|\tau|$ & & & & & & 1420 & & & & \\
\hline
\end{tabular}

\section{Celtis australis $I_{\text {. }}$.}

obere Grenze

\begin{tabular}{|c|c|c|c|c|c|c|c|}
\hline Summe & 278 & 155 & 418 & 261 & 325 & 302 & 1739 \\
\hline Anzahl & 1 & 1 & 2 & 1 & 1 & 1 & 7 \\
\hline Mittel & & & & & & & 248 \\
\hline
\end{tabular}

\section{Laurus nobilis I.}

obere Grenze

Max. 


\begin{tabular}{|c|c|c|c|c|c|c|c|c|c|c|c|c|}
\hline \multirow{2}{*}{ 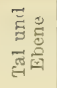 } & \multirow{2}{*}{ 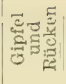 } & \multirow{2}{*}{$\begin{array}{l}\text { Mittel- } \\
\text { wert }\end{array}$} & \multicolumn{9}{|c|}{ Berglehnen nach ihrer Exposition } & \multirow{2}{*}{$\begin{array}{c}\text { Im } \\
\text { Allge- } \\
\text { meinen }\end{array}$} \\
\hline & & & $\mathrm{N}$ & No & 0 & SO & $\mathrm{S}$ & SW & W & NW & $\begin{array}{l}\text { Mittel- } \\
\text { wert }\end{array}$ & \\
\hline
\end{tabular}

\section{Hex aquifolium L.}

a) intere Grenze

\begin{tabular}{l|l} 
Min. & $\mid 261$
\end{tabular}

b) obere Grenze

Max.

890

\section{Rhammus eathartica $I_{\text {. }}$}

obere Grenze

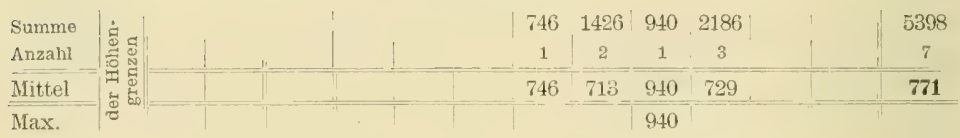

\section{Rlıammus alpina $\mathrm{L}$.}

a) untere Grenze

\begin{tabular}{|c|c|c|c|c|c|c|c|c|}
\hline Summe & & 1040 & 507 & 1520 & 1086 & 500 & 680 & 5333 \\
\hline Anzahl & 总 & 2 & 2 & 2 & 2 & 1 & 1 & 10 \\
\hline Mittel & $\begin{array}{l}E \\
5 \\
5\end{array}$ & 520 & 25.4 & T(ii) & 513 & 500 & 680 & 533 \\
\hline Min. & 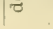 & & 244 & & & & & \\
\hline
\end{tabular}

b) obere Grenze

\begin{tabular}{|c|c|c|c|c|c|c|c|c|}
\hline Summe & & 2826 & 2301 & 1588 & 1428 & 2956 & 1214 & 12313 \\
\hline Anzahi & 胥 & 2 & 2 & 1 & 1 & 2 & 1 & 9 \\
\hline Mittel & $=$ & 1413 & 1151 & 1588 & 1428 & 1478 & 1214 & 1368 \\
\hline Max. & $\approx$ & & & 1588 & & & & \\
\hline
\end{tabular}

Paliurus aculeatus Lam.

obere Grenze

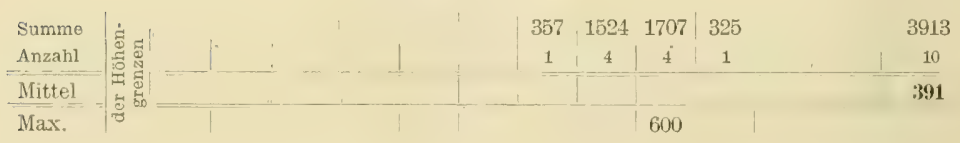




\begin{tabular}{|c|c|c|c|c|c|c|c|c|c|c|c|c|}
\hline \multirow{2}{*}{ 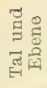 } & \multirow{2}{*}{ 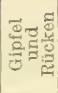 } & \multirow{2}{*}{$\begin{array}{l}\text { Mittel- } \\
\text { wert }\end{array}$} & \multicolumn{9}{|c|}{ Berglehnen nach ihrer Exposition } & \multirow{2}{*}{$\begin{array}{c}\text { Im } \\
\text { Allge- } \\
\text { meinen }\end{array}$} \\
\hline & & & $\mathrm{N}$ & No & 0 & $\mathrm{SO}$ & S & SW & W & NW & $\begin{array}{c}\text { Nittel- } \\
\text { wert }\end{array}$ & \\
\hline
\end{tabular}

Cornus mas $\mathbf{L}$.

obere Grenze

\begin{tabular}{l|c|c|c|c|c|c|c|c|c} 
Summo & 557 & 3149 & 818 & 938 & 741 & 2258 & 2214 & 2939 & $136+4$ \\
Anzahl \\
\hline Mittel
\end{tabular}

Amelanchier oralis Med. (Aronia rotundifolia Pers.)

obere cirenze

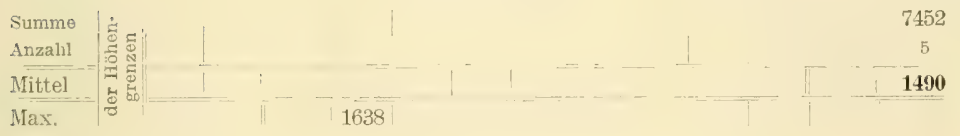

Cytisus Laburum L. (Laburnum rulgare Griseb.)

obere Grenze

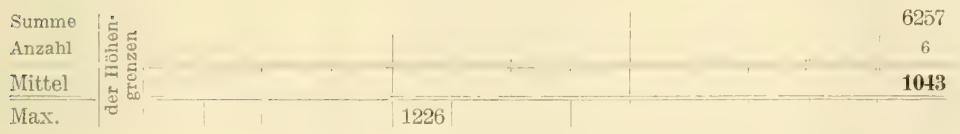

Olea enropaea $\mathbf{I}$.

obere Grenze

$\operatorname{Max}$

Phillyrea latifolia L.

obere Grenze

Max. 


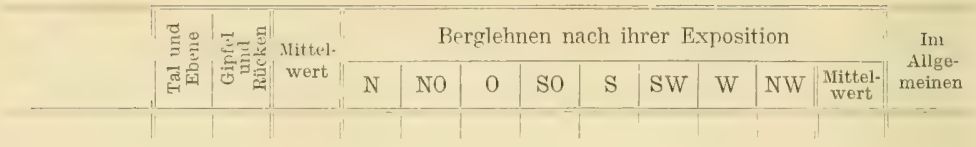

\section{Sambucus nigra $\mathbf{L}$.}

a) untere Grenze an der Meerseite

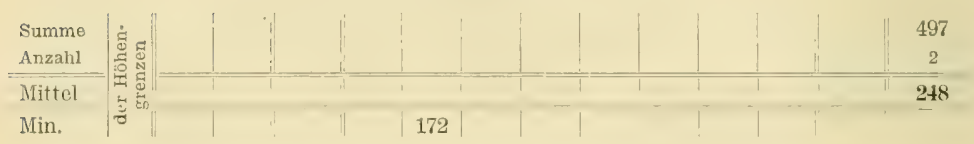

b) obere Grenze

\begin{tabular}{|c|c|c|c|c|c|c|c|c|c|}
\hline Summe & & 3953 & 759 & 3744 & 719 & 1092 & 845 & 887 & 11999 \\
\hline Anzahl & $\underset{\mathrm{D}}{\mathrm{D}}$ & 4 & 1 & 4 & 1 & 1 & 1 & 1 & 13 \\
\hline Mittel & $=\vec{a}$ & & & & & & & & 923 \\
\hline Max. & $1 \%$ & 1201 & & & & & & & \\
\hline
\end{tabular}

\section{Sambucus racemosa L.}

a) untere Grenze

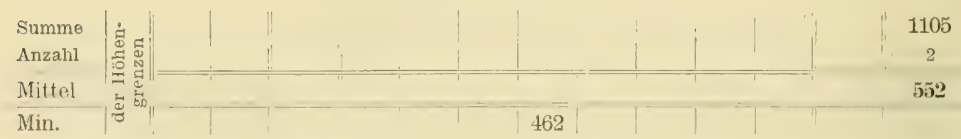

b) obere Grenze

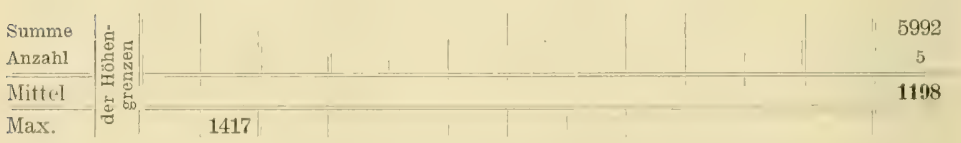

\section{Viburnum opulus $\mathbf{L}$.}

obere Grenze

\begin{tabular}{|c|c|c|c|c|c|c|c|c|}
\hline Summe & & 596 & 1030 & 470 & 1166 & 482 & 344 & 4088 \\
\hline Anzahl & 送 & 1 & 2 & 1 & 2 & 1 & 1 & 8 \\
\hline Mittel & 25 & 5446 & 515 & 470 & $5 \$ 3$ & 482 & 344 & 511 \\
\hline Max. & $\sigma^{\circ}$, & & & & 587 & & & \\
\hline
\end{tabular}




\begin{tabular}{|c|c|c|c|c|c|c|c|c|c|c|c|c|}
\hline \multirow{2}{*}{ 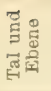 } & \multirow{2}{*}{ 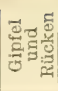 } & \multirow{2}{*}{$\begin{array}{l}\text { MitteI- } \\
\text { wert }\end{array}$} & \multicolumn{9}{|c|}{ Berglehnen nach ihrer Exposition } & \multirow{2}{*}{$\begin{array}{c}\operatorname{Im} \\
\text { Allge- } \\
\text { meinen }\end{array}$} \\
\hline & & & $\mathrm{N}$ & No & 0 & So & S & SW & W & NW & $\begin{array}{l}\text { Mittel- } \\
\text { wert }\end{array}$ & \\
\hline
\end{tabular}

Vitis vinifera $L$.

obere Grenze

\begin{tabular}{|c|c|c|c|c|c|c|c|c|}
\hline Summe & \multirow{4}{*}{ 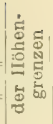 } & 770 & 583 & $431^{\prime}$ & 538 & 978 & 394 & 3694 \\
\hline Anzahl & & 2 & 1 & 1 & 1 & 2 & 1 & 8 \\
\hline Mittel & & 355 & 583 & 131 & 538 & 489 & 394 & 462 \\
\hline Max. & & & & & & 604 & & \\
\hline
\end{tabular}




\section{Erdélyer (Siebenbiirger) Becken.}

(Die höchsten Ansteigungen 6-800 m.)

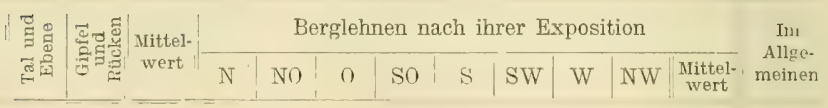

Fagus silvatica L.

a) vereinzelt, untere Grenze

\begin{tabular}{|c|c|c|c|c|c|c|c|c|c|c|c|c|}
\hline Summe & & 380 & 330 & 710 & 1260 & 732 & 418 & 471 & 400 & 300 & 3581 & $4: 291$ \\
\hline Anzahl & 댕 & 1 & 1 & 2 & 3 & 2 & 1 & 1 & 1 & 1 & 9 & 11 \\
\hline Mittiel & I & 380 & 330 & 355 & 420 & 366 & 418 & 471 & 400 & 300 & 398 & 390 \\
\hline
\end{tabular}

b) bestandbildend. untere Grenze

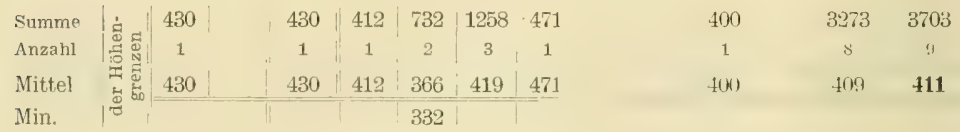

\section{Gross- I. Klein-Alföld ('Tiefland).}

Fagus silvatica $\mathbf{L}$.

Untere Grenze, Minimum: $82 \mathrm{~m}$. 


\section{ïbersicht \\ der rertikalen Verbreitungsgrenzen.}

In diesen Tabellen haben wir die nach Gebirgsgegenden bereits angeführten mittleren Höhengrenzen auf die nächsten 10-er abgerundet und nach Holzarten gruppiert. Diese \%usammenstellung bietet ein leicht îbersichtliches Bild von den Verhältnissen der vertikalen Verbreitung der angeführten Holzarten im gesamten Staatsgebiete.

Arten, die nur in einzelnen Gegenden vorkommen, deren l'egetationsgrenzen also zur Vergleichung ungeeignet sind, wurden hier nicht aufgenommen. Diese behandeln wir in den am Begimm des Bandes befindlichen Tabellen: „Die Grenzen der vertikalen Verbreitung", während wir bezüglich jener Arten, deren vertikale Verbreitung wir nicht in allen Gebirgsgegenden klären konnten, nur im Textteile (Bd. I.) eingehen können. 


\section{Mittelwerte füir dlas Gebiet des ungarischen States.}

Wenn fragliche Art in irgend einem Gebiete nicht vorkommt, bezeichnen wir dies in den Rubriken mit vagrechter Linie, wenn dieselbe ihre Höhengrenze nicht erreicht, mit Stern (*); wenn wir wegen mangelhafter Beobachtung - welcher Umstand bereits auch in sehr vereinzeltem Vorkommen der bezeichneten Art seine natürliche Begründung hat - einen verlüsslichen Durch . schnitt nicht bilden konnten, mit einem Punkt.

Die Summe der gesamten Höhengrenzen dividiert durch die Anzahl der Erhebungen, gebon den Mittelecert fiir den gainzen Staat.

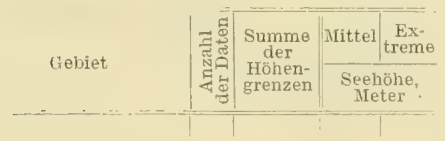

Picea excelsa lk.

a) Vereinzelt, untere tirenze

Nin.

Nordwestkarpathen. $|\mathbf{1 1}| \quad 4519 \quad \mathbf{4 1 0} \mid 302$

\begin{tabular}{|l|l|l|l|l|} 
Centralkarpathen. . & 54 & 28003 & $\mathbf{5 2 0}$ & 302
\end{tabular}

Nordostkarpathen . $|64| 40079$ i| 630 | 370

\begin{tabular}{l|l|l|l|l} 
Ostkarpathen ... & $56 \mid$ & 41335 & $\mathbf{7 4 0}$ & 522
\end{tabular}

\begin{tabular}{|l|l|l|l|l|} 
Siidkarpathen . . . & 52 & 41410 & 800 & 490
\end{tabular}

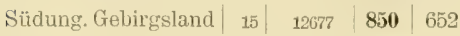

\begin{tabular}{|l|l|l|l|l} 
Bihargebirge. . . . & 88 & 25806 & $\mathbf{9 2 0}$ & 561
\end{tabular}

Ung. Mittelgebirge : $-1-1-1-$

Inselgebirge . . . $1-1-1-1-$

\begin{tabular}{l|l|l|l|l|l} 
Ungarische Alpen . & 8 & 3617 & $\mathbf{4 5 0}$ & 213
\end{tabular}

Kroatische Alpen - $|18| \quad 8594$ | $\mathbf{4 8 0} \mid 159$

Für das ganze

\begin{tabular}{l|l|l|l|l} 
Staatsgebiet & 306 & 206070 & $\mathbf{6 7 0}$ & 159
\end{tabular}

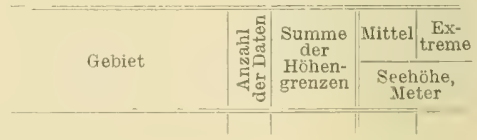

1) Bestanibisdend, untere (irenze Min. Nordwestkarpathen. $\mid$\begin{tabular}{l|l|l|l|}
$\mathbf{1 7}$ & 8742 & $\mathbf{5 1 0}$ & 332
\end{tabular} \begin{tabular}{l|l|l|l|l} 
Centralkarpathen. . & 60 & 38453 & $\mathbf{6 4 0}$ & 315
\end{tabular}

Nordostkarpathen .| 114| $82401 \quad|\mathbf{7 2 0}| 400$

\begin{tabular}{|l|l|l|l|l} 
Ostkarpathen... & 71 & 58689 & $\mathbf{8 3 0}$ & 523
\end{tabular} \begin{tabular}{|l|l|l|l|l|l} 
Südkarpathen . . . & 49 & 53303 & $\mathbf{1 0 7 0}$ & 554
\end{tabular}

\begin{tabular}{ll|l|l|l} 
Südung. Gebirgsiand & 6 & 6228 & $\mathbf{1 0 4 0}$ & 938
\end{tabular}

\begin{tabular}{ll|lll|l} 
Bihargebirge . . . & 42 & 45650 & $\mathbf{1 0 9 0}$ & 590
\end{tabular}

Ung. Mittelgebirge . $|-|-1|-|-$

Inselgebirge . . . . $|-|-1 \quad-\quad-$

\begin{tabular}{l|l|l|l|l} 
Ungarische Alpen . & 3 & 1591 & $\mathbf{5 3 0}$ & 436
\end{tabular}

\begin{tabular}{l|l|l|l|l} 
Kroatische Alpen . & $\mathbf{1 6}$ & $\mathbf{1 0 1 0 5}$ & $\mathbf{6 3 0}$ & 355 \\
\hline
\end{tabular}

\begin{tabular}{|l|ll|l|l|l|l|l|l|l|l|}
\hline Für das ganze & & & & & \\
\hline
\end{tabular} 


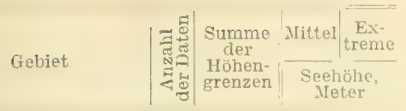

c) Geschlossener Bestand, obere Grenze

Nax.

Nordwestkarpathen. | 24| $31613|\mathbf{1 3 2 0}| 1459$

Centralkarpathen. : | $375|506720| 1350 \mid 1662$

\begin{tabular}{l|l|l|l|l|} 
Nordostkarpathen . & 425 & 583244 & $\mathbf{1 3 7 0}$ & 1589
\end{tabular}

\begin{tabular}{|l|l|l|l|l|} 
Ostkarpathen $\ldots$ & 212 & 320537 & $\mathbf{1 5 1 0}$ & 1756
\end{tabular}

Südkarpathen ... | $202 \mid 347382$ 1680| 1858

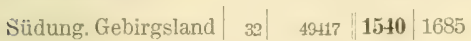

Bihargebirge. . . . $69\left|{ }_{105562}\right| \mathbf{1 5 3 0} \mid 1697$

Ung. Mittelgebirge . $|-|-\quad-1-$

Inselgebirge ... $\mid-1-1-1-$

Ungarische Alpen . $|*| *|*| *$

Kroatische Alnen . $\left|{ }_{14}\right| \quad 18676|\mathbf{1 3 3 0}| 1504$

Für das ganze \begin{tabular}{l|l|l|l|l} 
Staatsgebiet & 1358 & 1963151 & $\mathbf{1 4 5 0}$ & 1858
\end{tabular}

d) Gbere Walderenze

Nordwestkarpathen. $\mid$ 23 $\mid$ 3950 $1420 \mid 1500$

\begin{tabular}{ll|ll|l|l|} 
Centralkarpathen . $\quad 410 \mid$ & 596609 & $\mathbf{1 4 6 0}$ & 1695 \\
\hline
\end{tabular}

Nordostkarpathen . $|335| 492012$

Ostkarpathen .. . | $146 \mid 234687$

Südkarpathe11 ... | 195| 340582

$1470 \mid 161 \pm$

$\mathbf{1 6 1 0} \mid 1771$.

$1750 \mid 1880$

Süchung. Gebirgsland $|79| 125768$

$\mathbf{1 5 9 0} \mid 1753$

Bihargebirge. . . . $\mid$ 6s $\mid 107606$

$1580 \mid 1754$

Ung. Mittelgebirge $\cdot|-|-$

Inselgebirge $\ldots .|-|-\ldots-1-$

Ungarische Alpen .| * * * * * *

\begin{tabular}{l|l|l|l|l} 
Kroatische Alpen. & 4 & 5675 & $\mathbf{1 4 2 0}$ & 1440 \\
\hline
\end{tabular}

Für das ganze

Staatsgebiet $1260 \mid 1935508:$

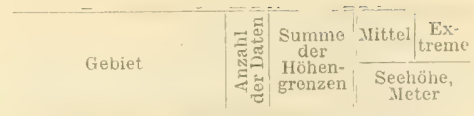

e) Verkrïppelt, obere trenze

Nordwestkarpathen. $|*| \quad * \quad * \quad$ Max.

\begin{tabular}{ll|l|l|l|l} 
Centralkarpathen. . & 15 & 27389 & $\mathbf{1 8 3 0}$ & 2057
\end{tabular}

\begin{tabular}{l|l|l|l|l} 
Nordostkarpathen . & 52 & 91892 & $\mathbf{1 7 7 0}$ & 1930
\end{tabular}

Ostkarpathen …|24 $46765 \quad \mathbf{1 9 5 0} \mid 2026$

Südkarpathen ... $\left|{ }_{50}\right|$ 97004 $|\mathbf{1 9 4 0}| 2200$

Südung. Gebirgsland $|9|{ }_{16160} \quad \mathbf{1 8 0 0} \mid 2100$

Bihargebirge. . . | $11 \mid 19412$ |1770 1830

Ung. Mittelgebirge $\cdot|-|-1-1-$

Inselgebirge ... $|-|-1-1-$

Ungarische Alpen . $* \mid$ * $\quad *|*|$ *

\begin{tabular}{l|l|l|l|l} 
Kroatische Alpen & 12 & 19538 & $\mathbf{1 6 3 0}$ & 1699
\end{tabular}

Für das ganze

Staatsgebict! $173 ! 318180 \quad|\mathbf{1 8 4 0}| 2200$

Lbies pectinata DC. (A. alba Mill.)

a) Cotere (irenze Min

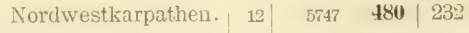

\begin{tabular}{l|l|l|l|l} 
Centralkarpathen. . & 36 & 14591 & $\mathbf{4 1 0}$ & 215
\end{tabular}

\begin{tabular}{ll|ll|l} 
Nordostkarpathen . 110 & 69118 & $\mathbf{6 3 0}$ & 300
\end{tabular}

\begin{tabular}{lll|l|l} 
Ostkarpathen $\ldots$ & 49 & 37490 & $\mathbf{7 7 0}$ & 545
\end{tabular}

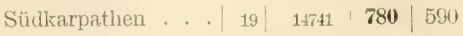

\begin{tabular}{ll|l|l|l} 
Sutdung. Gebirgsland & 43 & 28632 & $\mathbf{6 7 0}$ & 327
\end{tabular}

Bihargebirge. . . $|27| 22680 \quad 840 \mid 620$

Ung. Mittelgebirge $\cdot|-|-\quad-\left.\right|^{--}$

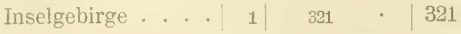

Ungarische Alpen . $\left|{ }_{10}\right| \quad \mathbf{4 1 8 1} \quad \mathbf{4 2 0} \mid 218$

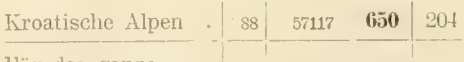

F'iir das ganze

Staatsgebiet $|395| 254618 \quad 640 \mid 204$ 


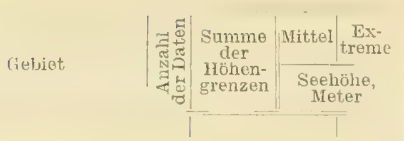

b) Bestandbiltead, obere (irenze

Nordwestkarpathen.

\begin{tabular}{|l|l|l|l|l|l|l|l|l|l|} 
Centralkarpathen. . & 114 & 122801 & 1080 & 1335
\end{tabular}

Nordostisarpathen . 34 | 31474 || $\mathbf{9 3 0} 1326$

\begin{tabular}{ll|l|l|l|l} 
Ostkarpathen & $\ldots$ & 20 & 25967 & 1300 & 1456
\end{tabular}

Südkarpathen ... $\quad 3|3929 \quad| \mathbf{1 3 1 0} \mid 1339$

Sïdung. Gebirgsland |

Bihargebirge. .. $\left|{ }_{6}\right| 6_{690}|\mathbf{1 1 5 0}| 1270$

Ung. Mittelgebirge . $|-|-1-1-$

Inselgebirge ... $*|*| *|*| *$

Ungarische Alpen . $*$ * $\quad * \quad * \quad$ *

\begin{tabular}{ll|l|l|l|} 
Kroatische Alpen & 22 & 20551 & 1210 & 1406
\end{tabular}

Für das ganze

Staatsgebiet $199|217642| 1090 \mid 1456$

c) Vereinzelt, obere tirenze

Max

\begin{tabular}{l|rr|r|} 
Nordwestkarpathen. | 18 & 21233 & $\mathbf{1 1 8 0}$ & 1281
\end{tabular}

\begin{tabular}{|l|l|l|l|l|l|}
\hline Centralkarpathen. . & 262 & 330778 & $\mathbf{1 2 6 0}$ & 1562
\end{tabular}

\begin{tabular}{|l|l|l|l|l|} 
Nordostkarpathen . 294 & 346972 & $\mathbf{1 1 8 0}$ & $142\}$
\end{tabular}

\begin{tabular}{|l|l|ll|l} 
Ostkarpathen . . . & 89 & 118033 & $\mathbf{1 3 3 0}$ & 1712
\end{tabular}

Sïdkarpathen ... $91 \mid 124782$ |1370 $\mid 1734$

\begin{tabular}{l|r|r|r|r} 
Südung. Gebirgsland & 35 & 47303 & $\mathbf{1 3 5 0}$ & 1545
\end{tabular}

\begin{tabular}{|l|l|l|l|l|l|} 
Bihargebirge. . . . & 76 & 101031 & $\mathbf{1 3 3 0}$ & 1560
\end{tabular}

Ung. Mittelgebirge $\cdot|-|-\mid-1-$

Inselgebirge .... $*|*| * \mid *$

Ungarische Alpen . $|*|$ * , * $\mid$ *

\begin{tabular}{l|ll||l|l|} 
Kroatische Alpen . & 35 & 46976 & $\mathbf{1 3 4 0}$ & 1617
\end{tabular}

Für das ganze

Staatsgebiet $900|1137108| 1260 \quad 1734$

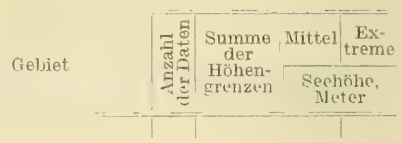

d) Veckrippelf, obere (irenze

\begin{tabular}{|c|c|c|c|}
\hline Nordwestkarpathen. & $1 \mid$ & 1300 & \\
\hline Centralkarpathen. . & 81 & 111282 & 1370 \\
\hline Nordostkarpathen . & 53 & 72850 & 1380 \\
\hline Ostkarpathen & 13 & 19163 & 1470 \\
\hline Süclkarpathen . . & 12 & 17800 & 1480 \\
\hline Südung. Gebirgsland & 9 & 12622 & 1400 \\
\hline Bihargebirge . . . & $5 \mid$ & 6943 & 1390 \\
\hline Ung. Mittelgebirge & -1 & - & - \\
\hline Inselgebirge . . . & * & * & * \\
\hline Ungarische Alpen & * & * & * \\
\hline Kroatische Alpen & 8 & 11335 & 1420 \\
\hline $\begin{array}{l}\text { ir das ganze } \\
\text { Staatsgebiet }\end{array}$ & 182 & 253995 & 1390 \\
\hline
\end{tabular}

\section{l’inus silvestris $\mathbf{L}$.}

a) Intere fireuze

Min.

\begin{tabular}{l|l|l|l|l} 
Nordwestkarpathen. & 4 & $1355,3 \mathbf{H 0}$ & $\mathbf{3 1 2}$
\end{tabular}

\begin{tabular}{l|l|l|l|l} 
Centralkarpathe11. . & 18 & 7412 & $\mathbf{4 1 0}$ & 232
\end{tabular}

\begin{tabular}{l|l|l|l|l|l} 
Nordostkarpathen . & 1 & 603 & $\cdot$ & 602
\end{tabular}

\begin{tabular}{|l|l|l|l|} 
Ostkarpathen $\ldots$ & 1 & $510 \quad$ & 510
\end{tabular}

\begin{tabular}{|l|l|l|l|l} 
Südkarpathen . . & 7 & 4128 & $\mathbf{6 3 0}$ & 476
\end{tabular}

\begin{tabular}{l|l|l|l|} 
Südung, Gebirgsland . . . . (700) & . .
\end{tabular}

Bihargebirge .... . . . .

Ung. Mittelgebirge . $|-|-1-1-$

Inselgebirge ....

\begin{tabular}{l|l|l|l|l} 
Ungarische Alpen . & 24 & 7217 & 300 & 185
\end{tabular}

\begin{tabular}{|l|l|l|l|l|}
\hline Kroatische Alpen & 12 & 3707 & $\mathbf{3 1 0}$ & $\mathbf{1 6 4}$ \\
\hline
\end{tabular}

\begin{tabular}{ll|l|l|l|l|l|l|l|l|}
\hline Für das ganze & & & \\
\hline
\end{tabular} 


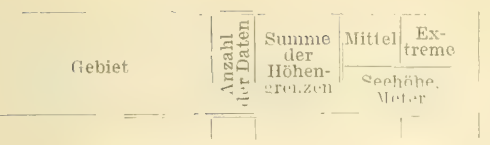

b) Bestandbildend, obere tremze

\begin{tabular}{|c|c|c|c|c|}
\hline Nordwestkarpathen. & 8 & 54 iff & 680 & $\begin{array}{l}\operatorname{Max} \\
829\end{array}$ \\
\hline Centralkarpathen. . & 2 & 2265 & 1130 & 1156 \\
\hline Nordostkarpathen . & . & . & . & . \\
\hline Ostkarpathen ... & 3 & 2260 & 1130 & 1210 \\
\hline Südkarpathen . . . & 1 & 1199 & . & 1190 \\
\hline Siiclung. Gebirgsland & . & . & - & . \\
\hline Bihargebirge . . . . & . & . & . & $\cdot$ \\
\hline Ung. Mittelgebirge . & - & - & - & - \\
\hline Inselgebirge . . . . & - & - & - & - \\
\hline Ungarische Alpen . & $*$ & * & $*$ & * \\
\hline Kroatische Alpen . & . & . & . & - \\
\hline $\begin{array}{l}\text { Für das ganze } \\
\text { Staatsgebiet }\end{array}$ & 13 & 11181 & 860 & 1210 \\
\hline
\end{tabular}

c) Vereinzelt, obero (drouze

\begin{tabular}{l|l|l} 
Nordwestkarpathen. & 9 & 7975
\end{tabular}

Centralkarpathen. . $|27| 33006$

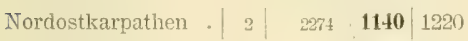

\begin{tabular}{ll|l|l|l|l|l|} 
Ostlkarpathen... & 4 & 5085 & $\mathbf{1 2 7 0}$ & 1405
\end{tabular}

\begin{tabular}{ll|l|l|l|l} 
Suidkarpathen $\ldots$ & 17 & 24029 & $\mathbf{1 4 1 0}$ & 1650
\end{tabular}

Süidung. Gebirgsland| .

\begin{tabular}{|l|l|l|l|l|l|l|l|l|l|} 
Bihargebirge . . . & 1 & 1353 & 1353
\end{tabular}

Ung. Mittelgebirge . $|-|-1-1-$

Inselgebirge .... . $-1-1-1-$

Ungarische Alpen . . . . | | | (755)

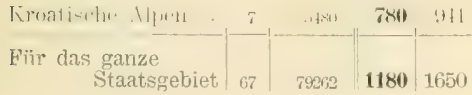

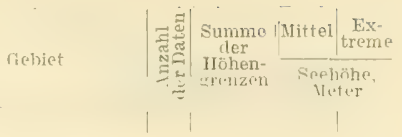

\section{Piuns nigra Iru.}

a) Untere firenze Min. Novilwestlsarpathen. $\mid-1+\ldots+1$ Centralkarpathen. . $|-|-$ Nordostkarpathen Ostkarpathen Sïdkarpathen ... . $-1 \quad-$

\begin{tabular}{l|l|l|l|l} 
Südung. Gebirgsland & 8 & 2808 & $\mathbf{3 5 0}$ & 185
\end{tabular} Bihargebirge . . . | - - $1-1+$ Ung. Mittelgebirge $\cdot 1-1-1,-$ Inselgebirge . . . $\mid-1-1,-$ Ungarische Alpen : $-1+\ldots \quad-\mid-$

\begin{tabular}{l|l|l|l|l} 
Kroatische Alpen & 5 & 1730 & $\mathbf{3 5 0}$ & 0 \\
\hline Fïr das ganze & & & & \\
Staatsgebiet & 13 & 4538 & $\mathbf{3 5 0}$ & 0
\end{tabular}

b) Baum form, obece (trenz.

I

Nortivestlearpathen. Nordwestkarpathen. $|-|-1-1$
Centralkarpathen. . | $-1-$ Nordostikarpathen : $|-|-i|-|-$ Ostkarpathen ... . -1 Sïdkarpathen . . . $|-|-1-1=$ Siidung. Gebirgsland $|+| 3900 \quad 980 \mid 1109$ Bihargebirge . . . Ung. Mittelgebirge . $\mid-1+$ - $_{-1}$ Inselgebirge . . . . $|-|-1-1-$ Ungarische Alpen $\cdot|-|-1=$

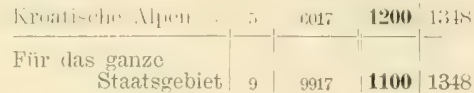


Geliet

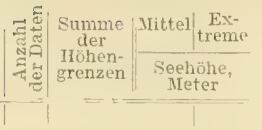

\section{Pinus Cembra $L_{\text {. }}$}

a) Intere Grenze

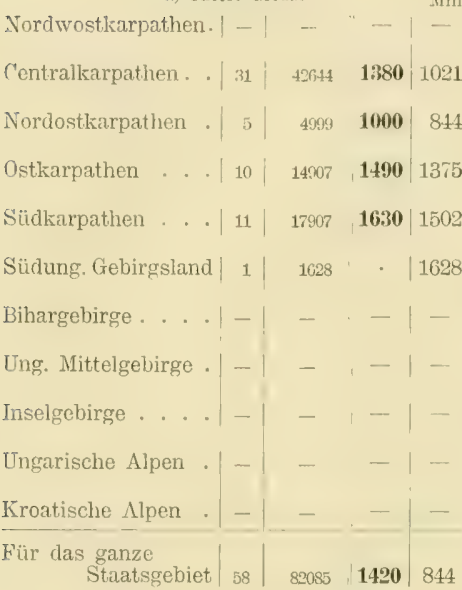

b) Манаtorna, obere frenze

\begin{tabular}{|c|c|c|c|c|}
\hline Nordwestkarpathen. & -1 & - & - & Max. \\
\hline Centralkarpathen. . & $57 \mid$ & 01801 & 1610 & 1762 \\
\hline Nordostkarpathen . & 4 & 5809 & $1450 \mid$ & 1473 \\
\hline Ostkarpathen ... & 13 & 22132 & 1700 & 1771 \\
\hline Südkarpathen . . . & 39 & 71772 & 1840 & 1986 \\
\hline Siidung. Gebirgsland | & 6 & 11006 & $|1810|$ & 1870 \\
\hline Bihargebirge. . . | & -1 & - & - & - \\
\hline Ung. Alittelgebirge . & - & - & - & - \\
\hline Inselgebirge . . . & - & - & - & - \\
\hline Ungarische Alpen . & - & - & - & - \\
\hline Kroatische Alpen & - & - & - & - \\
\hline $\begin{array}{r}\text { Fiur las ganze } \\
\text { Staatsgebiet }\end{array}$ & 119 & 202647 & 1700 & 1986 \\
\hline
\end{tabular}

Giebiet

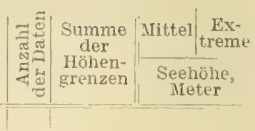

c) Verkrïppelt, obere firenze

Max.

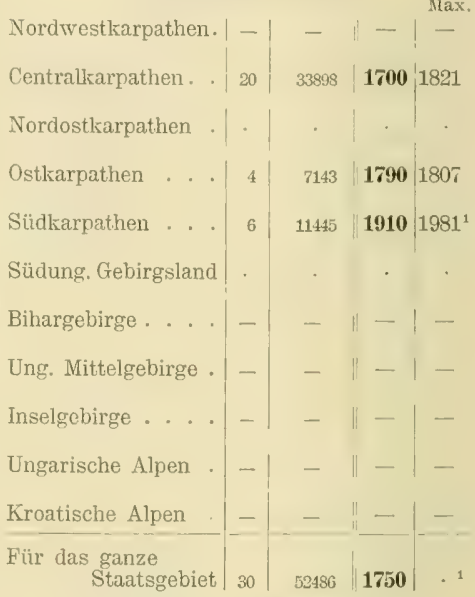

Pinus montana Mill.

a) Untere Grenze

Min.

\begin{tabular}{l|l|l||l|l|l|l|l} 
Nordwestkarpathen. & 7 & 9794 & $\mathbf{1 4 0 0}$ & $\mathbf{1 3 3 5}$
\end{tabular}

\begin{tabular}{l|l|l|l|l|l} 
Centralkarpathen. & 72 & 91593 & $\mathbf{1 2 7 0}$ & 752
\end{tabular}

\begin{tabular}{ll|l|l||l|l} 
Nordostkarpathen. & 79 & 112892 & 1430 & 1190
\end{tabular}

\begin{tabular}{ll|l|l|l|l} 
Ostkarpathen $\ldots$ & 32 & 50197 & $\mathbf{1 5 7 0}$ & 1328
\end{tabular}

\begin{tabular}{|l|l|l|l|l} 
Südkarpathen . . . & 21 & 32543 & $\mathbf{1 5 5 0}$ & 1210
\end{tabular}

\begin{tabular}{l|l|l|l|l|l} 
Südung. Gebirgsland & 9 & 14415 & $\mathbf{1 6 0 0}$ & 1291
\end{tabular}

Bihargebirge . . $\mid$\begin{tabular}{l|l|l|l|l|} 
& 5 & 7447 & $\mathbf{1 4 7 0}$ & 1200
\end{tabular}

Ung. Mittelgebirge $\cdot 1-1-1-1-$

Inselgebirge . . . $1-1-;-1-$

Ungarische Alpen : $|-|+i \mid-1-$

Kroatische Alpen · | 23 $31616 \quad \mathbf{1 3 8 0} \mid 1096$

Für das ganze

Staatsgebiet $|248| 350397 \quad \mathbf{1 4 1 0} \mid 752$ 


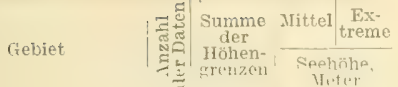

b) Obere Grenze

\begin{tabular}{ll|l|l|l|l|} 
Nordwestkarpathen. & 1 & $1672 \quad$ & 1672
\end{tabular}

\begin{tabular}{l|l|ll|l} 
Centralkarpathen. & 77 & 149176 & $\mathbf{1 9 4 0}$ & 2140
\end{tabular}

\begin{tabular}{l|l|l|l|l} 
Nordostkarpathen . & 93 & 43015 & $\mathbf{1 8 7 0}$ & 2000
\end{tabular}

Ostkarpathen ... . $22|43332| \mathbf{1 9 7 0} \mid 214 \overline{7}$

\begin{tabular}{ll|ll|l|l|l|l|l|l|} 
Südkarpathen $\ldots 8876$ & $\mathbf{2 1 5 0}$ & 2305
\end{tabular}

Südung. Gebirgsland $|*|$ * * * $\mid$ *

\begin{tabular}{|l|l|l|l|l} 
Bihargebirge. . . . & 4 & $7104, \mathbf{1 7 8 0}$ & 1838
\end{tabular}

Ung. Mittelgebirge . $|-|+\ldots+1-$

Inselgebirge . . . $|-|-\quad-1-$

Ungarische Alpen . $|+| \quad \ldots \quad-y_{-}$

\begin{tabular}{l|l|l|l|l} 
Kroatische Alpen & $*$ & $*$ & $*$ & $*$ \\
\hline Für das ganze & & & &
\end{tabular}

\begin{tabular}{l|l|l|l|l} 
- Staatsgebiet & 159 & 313175 & $\mathbf{1 9 7 0}$ & 2305
\end{tabular}

Larix europaea BC. (L。 decidua Mill.)

a) Intere lirenze

Nordwestkarpathen. $|\cdot|$.

\begin{tabular}{l|l|ll|l} 
Centralkarpathen. . & 15 & $\$ 94$ & $\mathbf{6 5 0}$ & 398
\end{tabular}

\begin{tabular}{l|l|ll|l} 
Nordostkarpathen. & 4 & 3660 & $\mathbf{9} 20$ & 760
\end{tabular}

Ostkarpathen ... $\mid-1+\ldots-1=$

Südkarpathen . . . $|1| 1325 \quad \cdot \mid 1325$

Südung. Gebirgsland $|-|+1-1-$

Bihargebirge... . 1 | ri35 * $\mid 635$

Ung. Mittelgebirge $\cdot \mid-1+$

Inselgebirge . . . $\mid-1-$

Ungarische Alpen . $\mid$\begin{tabular}{l|l|l|l|}
4 & 1763 & $\mathbf{4 1 0}$ & 350
\end{tabular}

Kroatische Alpen $: 1-1-1-1=$

\begin{tabular}{c|c|cc|c} 
Fiir das ganze & & & & \\
Staatsgebiet & 35 & 17076 & $\mathbf{6 8 0}$ & 350
\end{tabular}

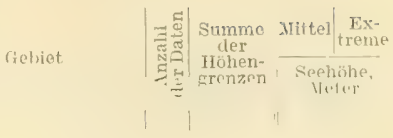

1) Baumform, obere trenze

Nordwestkarpathen. | .

\begin{tabular}{ll|l|l|l|l} 
Centralkarpathen. . & 94 & 138095 & $\mathbf{1 4 7 0}$ & 1697
\end{tabular}

Norlostkarpathen . $\quad 3 \mid$\begin{tabular}{lll|l}
3 & 3906 & $\mathbf{1 3 1 0}$ & 1412
\end{tabular}

Ostkarpathen ... $|-|-1-$

Stidkarpathen . . . $|3| \quad 5491 \quad \mathbf{1 8 3 0 |} \mid 1890$

Südung. Gebirgsland $1-1-1-1-$

Bihargebirge... | $1 \mid 1352$ | $\mid 1352$

Ung. Mittelgebirge . $|-|-1-1-$

Inselgebirge . . . $\mid-i=$

Ungarische Alpen . . . . . | (755)

Kroatische Alpen . $1-1+-1-1-$

\begin{tabular}{l|l|l|l|l} 
Für das ganze & & & & \\
Staatsgebiet & 101 & 149064 & $\mathbf{1 4 8 0}$ & 1890
\end{tabular}

c) Verkrippelt, ohere tirenze

Nordwestkarpatien. $\cdot|\cdot| \cdot \mid$,

\begin{tabular}{ll|l|l|l|l} 
Centralkarpathen. & 25 & 40430 & $\mathbf{1 6 2 0}$ & 1775
\end{tabular}

Nordostkarpathen .

Ostkarpathen ... $\mid-1-1-1-$

\begin{tabular}{l|l|ll|l} 
Südkarpathen . . . & 1 & 2049 & ! * & 2049
\end{tabular}

Sülung. (iebirgsland $!-1-1-1-$

Bihargebirge. . .

Ung. Mittelgebirge . $|-|-d-1$

Inselgebirge . . . . - -1

Ungarische Alpen

Kroatische Alpen : - $\mid-1$ -

Für das ganze

Statsgebiet $\mid 26$ | $42479 \quad \mathbf{1 6 3 0} \mid 2049$ 


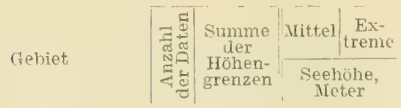

Taxus haceata L.

a) Untere Grenze

Nordwestkarpathen

Centralkarpathen. . $|13| \quad 8730$

Nordostkarpathen . $\mid$\begin{tabular}{l|l}
6 & 3989
\end{tabular}

Ost.karpathen ... . $1 \mid 490$

Südkarpathen ... 1 | 1000

Sïdung. Gebirgsland | $3 \mid 641$

Bihargebirge . . . | $1 \mid 250$

Ung. Mittelgebirge . | $1 \mid$ 301

Inselgehirge .... . $|-|$

Ungarische Alpen . | $1 \mid 498$

\begin{tabular}{l|l|} 
Kroatische Alpen : & 6 \\
\hline Fuir das ganze & \\
Staatsgebiet & 33
\end{tabular}

b) Ohere firenze

Nordwestkarpathen. | .

\begin{tabular}{l|l|l} 
Centralkarpathen . . & 18 & 17918
\end{tabular}

1000 : 1300

Nordostkarpathen , $|20| 21513, \mathbf{1 0 8 0} 1236$

Ostkarpathen ... $|1| 1436, \ldots \mid 1436$

\begin{tabular}{ll|l|l|l|l} 
Sïdkarpathen... & 2 & 2818 & $\mathbf{1 4 1 0}$ & 1500
\end{tabular}

Südung. Gebirgsland $|4| \quad$ 410s $\quad, \mathbf{1 0 3 0} \mid 1197$

\begin{tabular}{ll|l|l|l|l} 
Bihargebirge . . . . & 1 & 1199 & . & 1199
\end{tabular}

Ung. Nittelgebirge . |

Inselgebirge

Ungarische Alpen

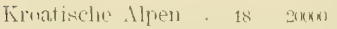

Fïir das ganze \begin{tabular}{l|l|l} 
Staatsgebiet & 61 & 68992
\end{tabular}

1110135 Einziger Standort.

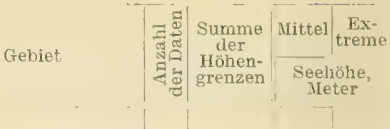

Juniperus communis $\mathbf{L}$.

bbere Grenze

Nax.

Nordwestkarpathen. 1 1 1436||$\cdot \mid 1436$

\begin{tabular}{l|l|l|l|l|l} 
Centralkarpathen..$~$ & 27 & 33980 & $\mathbf{1 3 6 0}$ & 1405
\end{tabular}

\begin{tabular}{l|l|l||l|l|} 
Nordostkarpathen. & 12 & 14457 & $\mathbf{1 2 1 0}$ & 1258
\end{tabular}

\begin{tabular}{l|l|l|l|l|l|} 
Ostkarpathen $\cdots$ & 28 & 38169 & $\mathbf{1 3 6 0}$ & 1521
\end{tabular}

\begin{tabular}{l|l|l|l|l} 
Siidkarpathen . . . & 1 & 922 & . & 922
\end{tabular}

Sïdung. Gebirgsland | 1 | 1380, . 1380

Bihargebirge. . . 1 | 1353 || 1353

\begin{tabular}{l|l|l|l|l} 
Ung. Mittelgebirge . & $*$ & $*$ & $*$ & $*$
\end{tabular}

Inselgebirge .... $|*| *|| * * \mid *$

Ungarische Alpen . $|*|$ * * $\quad$ * $\quad * \quad$ *

\begin{tabular}{l|l|l||l|l|} 
Kroatische Alpen . & 18 & 14312 & 800 & 1053
\end{tabular}

\begin{tabular}{|l|l|l|l|l|}
\hline Fiir das ganze & & & & \\
Staatsgebiet & 89 & 105999 & $\mathbf{1 1 9 0}$ & $\mathbf{1 5 2 1}$
\end{tabular}

\section{Juniperus nana Willd.}

a) Untere Grenze

Min.

\begin{tabular}{ll|lll|l} 
Nordwestkarpathen. & 5 & 6744 & $\mathbf{1 3 5 0}$ & 1299
\end{tabular}

Centralkarpathen. . $|23| 30233 \quad|\mathbf{1 3 1 0}| 1090$

\begin{tabular}{|l|l|l||l|l|} 
Nordostkarpathen . & 47 & 57851 & $\mathbf{1 2 3 0}$ & 703
\end{tabular}

\begin{tabular}{ll|l|l||l|l|} 
Ostkarpathen... & 54 & 70524 & $\mathbf{1 3 1 0}$ & 775
\end{tabular}

Südkarpathen . . . $|19| 22445 \quad \mid \mathbf{1 1 8 0} 622$

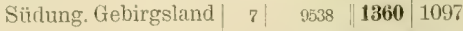

\begin{tabular}{|l|l|l||l|l} 
Bihargebirge . . . & 8 & 8338 & $\mathbf{1 0 4 0}$ & 648
\end{tabular}

Ung. Mittelgebirge . $\mid-1-1-1-$

Inselgebirge $\cdots \cdot|-|-|-|-$

Ungarische Alpen . $|-|-||-\mid-$

Krontische Alpen - $11 \quad 13120000$

\begin{tabular}{l|l|l|l|l} 
Für das ganze & & & & \\
Staatsgebiet & 174 & 218793 & $\mathbf{1 2 6 0}$ & 622
\end{tabular} 


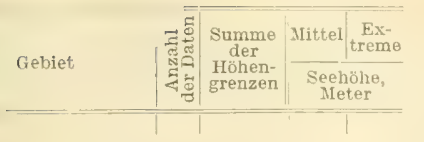

b) obere firenze

\begin{tabular}{|c|c|c|c|c|}
\hline & & & & $\operatorname{Max}$ \\
\hline Nordwestkarpathen. & * & * & * & * \\
\hline Centralkarpathen. . & 19 & 38068 & 2000 & 2140 \\
\hline Nordostkarpathen . & 15 & 28481 & 1900 & 2000 \\
\hline Ostkarpathen . . & 10 & 19961 & 2000 & 2140 \\
\hline Südkarpathen . . . & 23 & 48163 & 2110 & 2296 \\
\hline Südung. Gebirgsland & 2 & 4163 & 2080 & 2121 \\
\hline Bihargebirge. . . & * & 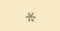 & * & * \\
\hline Ung. Mittelgebirge . & - & - & - & - \\
\hline Inselgebirge . . . & - & - & - & 一 \\
\hline Ungarische Alpen . & - & - & - & - \\
\hline Kroatische Alpen . & * & * & * & * \\
\hline $\begin{array}{l}\text { ür das ganze } \\
\text { Staatsgebiet }\end{array}$ & 69 & 139136 & $20 \geq 0$ & 2296 \\
\hline
\end{tabular}

\section{Fagus silvatica L.}

a) Vereinzelt, untere firenze

Nordwestkarpathen. $|26| \quad 8825|340| 200$

\begin{tabular}{l|l|l|l|l|} 
Centralkarpathen. . & 12 & 3945 & $\mathbf{3 3 0}$ & 200
\end{tabular}

Nordostkarpathen - $\mid$\begin{tabular}{l|l|l|l}
35 & $8898 \quad \mathbf{2 5 0}$ & 119
\end{tabular}

Ostkarpathen ... * * * * * * * * *

\begin{tabular}{|l|l|l|l|} 
Südkarpathen . . . & $*$ & $*$ & $*$
\end{tabular}

\begin{tabular}{l|l|l|l|l} 
Südung. Gebirgsland & 10 & 1820 & $\mathbf{1 8 0}$ & 52
\end{tabular}

\begin{tabular}{|l|l|l|l|l|} 
Bihargebirge . . . & 37 & 8922 & $\mathbf{2 4 0}$ & $\mathbf{1 6 1}$
\end{tabular}

\begin{tabular}{l|l|l|l|l|} 
Ung. Mittelgebirge . & 34 & 8953 & $\mathbf{2 6 0}$ & 116
\end{tabular}

\begin{tabular}{|l|l|l|l|l|} 
Inselgebirge . . . . & 63 & 13257 & $\mathbf{2 1 0}$ & 83
\end{tabular}

\begin{tabular}{|l|l|l|l|l} 
Hügell. beim Balaton & 3 & 600 & $\mathbf{2 0 0}$ & 200
\end{tabular}

\begin{tabular}{l|l|l|l|l} 
Ungarische Alpen . & 15 & 4684 & $\mathbf{3 1 0}$ & 130
\end{tabular}

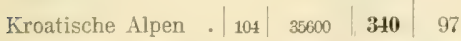

Erdélyer Becken . - $11 \mid$ 4291 $\quad 390 \mid 300$

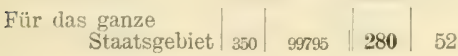

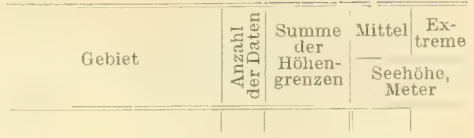

b) Bestandbildend, untere tirenze

\begin{tabular}{l|l|l|l|l|} 
Noritwestkarpathen. & 35 & 13228 & $\mathbf{3 8 0}$ & $\mathbf{3} 22$
\end{tabular}

\begin{tabular}{|l|l|l|l|l|l} 
Centralkarpathen. . & 39 & 12879 & $\mathbf{3 3 0}$ & 205
\end{tabular}

\begin{tabular}{l|l|ll|l|l|} 
Nordostkarpathen . & 87 & 30779 & $\mathbf{3 5 0}$ & 124
\end{tabular}

Ostkarpathen ... * * * $* 1 *$ *

Südkarpathen ... $|*| *|*| * \mid *$

\begin{tabular}{l|l|l|l|l} 
Südung. Gebirgsland & 18 & 3554 & $\mathbf{2 0 0}$ & 52
\end{tabular}

\begin{tabular}{|l|l|l|l|l|l|} 
Bihargebirge . . . . & 17 & 6127 & $\mathbf{3 6 0}$ & 161
\end{tabular}

\begin{tabular}{l|l|l|l|l} 
Ung. Mittelgebirge . & 25 & 7015 & $\mathbf{2 8 0}$ & $\mathbf{1 1 6}$
\end{tabular}

\begin{tabular}{|l|l|l|l|l|l} 
Inselgebirge $\ldots . .$. & 63 & 13257 & $\mathbf{2 1 0}$ & 83
\end{tabular}

\begin{tabular}{ll|l|l|l|l|} 
Hïgell. beim Balaton & 3 & 600 & $\mathbf{2 0 0}$ & 200
\end{tabular}

\begin{tabular}{l|l|l|l|l|l|} 
Ungarische Alpen & 16 & 6307 & 390 & 180
\end{tabular}

\begin{tabular}{l|l|l||l|l|} 
Kroatische Alpen . 139 & 67519 & $\mathbf{4 9 0}$ & 123
\end{tabular}

\begin{tabular}{l|l|l|l|l} 
Erclélyer Becken. . & 9 & 3703 & $\mathbf{4 1 0}$ & 332
\end{tabular}

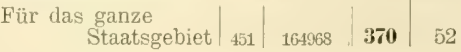

c) Bestandblliung im Allgemeinen, obere Grenze

Max.

Nordwestkarpathen. $\quad 4|\quad 4533| \mathbf{1 1 3 0} \mid 1223$

Centralkarpathen. . $|200| 249171 \quad \mathbf{1 2 5 0} \mid 1410$

\begin{tabular}{l|l|l|l|l|l|l|l|l|l|l|l|} 
Nordostkarpathen & 482 & 600175 & $\mathbf{1 2 5 0}$ & 1387
\end{tabular}

\begin{tabular}{|l|l|l|l|l|l}
\hline Ostkarpathen $\ldots$ & 117 & 158895 & $\mathbf{1 3 1 0}$ & 1505
\end{tabular}

\begin{tabular}{ll|l|l|l|l|l} 
Südkarpathen... & 105 & 138453 & $\mathbf{1 3 2 0}$ & 1560
\end{tabular}

\begin{tabular}{l|l|l|l|l|l} 
Südlung. Gebirgsland & 133 & 186887 & $\mathbf{1 4 1 0}$ & 1558
\end{tabular}

\begin{tabular}{|l|l|l|l|l|} 
Bihargebirge . . . & 101 & 131393 & $\mathbf{1 3 0 0}$ & 1499
\end{tabular}

\begin{tabular}{l|l|l|l|l} 
Ung. Mittelgebirge . & $*$ & $*$ & $*$ & $*$
\end{tabular}

Inselgebirge .... * * * $* 1 *$

Ungarische Alpen . $|*|$ * $\quad *|* *| *$

\begin{tabular}{|l|l|l||l|l|} 
Kroatische Alpen & 68 & 92793 & $\mathbf{1 3 7 0}$ & 1617
\end{tabular}

\begin{tabular}{l|l|l|l|l} 
Für das ganze & & & & \\
Staatsgebiet & $\mathbf{1 2 1 0}$ & 1556300 & $\mathbf{1 3 9 0}$ & 1617
\end{tabular} 
Gebiet

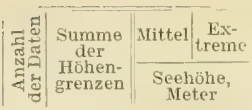

(1) IRestaudbilalend obere (irenze, als Walderenze

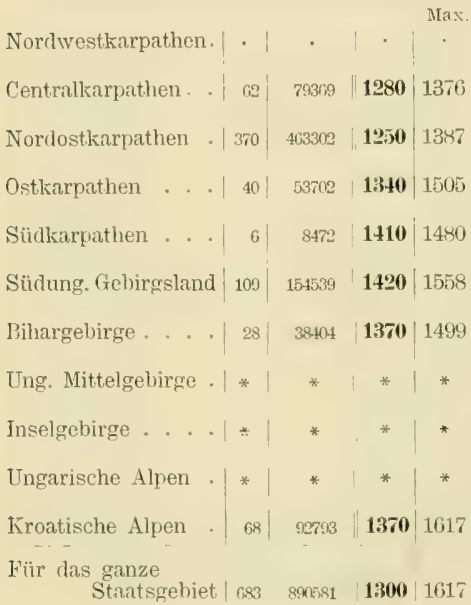

e) Bestandbildend olore Arenze, dariber Fichto

Max.

Nordwestkarpathen. $\left|\quad 4^{\prime} \quad 4.533\right| \mathbf{1 1 3 0} \mid 1223$

\begin{tabular}{l|l|l|l|l|l} 
Centralkarpathen. . & 138 & 169802 & $\mathbf{1 2 3 0}$ & 1410
\end{tabular}

Nordostkarpathen . $|112| 136873|\mathbf{1 2 2 0}| 138:$

Ostkarpathen . . . $77|99193| \mathbf{1 2 9 0} \mid 1490$

\begin{tabular}{ll|l|l|l|l|} 
Süidkarpathen $\cdots$ & 99 & 129081 & $\mathbf{1 3 1 0}$ & 1560
\end{tabular}

\begin{tabular}{ll|l|l|l|l|} 
Südung. Gebirgsland & 24 & 32348 & $\mathbf{1 3 5 0}$ & 1512
\end{tabular}

\begin{tabular}{|l|l|l|l|l|} 
Bihargebirge . . . . & 73 & 92989 & $\mathbf{1 2 7 0}$ & 1484
\end{tabular}

Ung. Mittelgebirge . $|*| *+* \mid *$

Inselgebirge .... * * * * $\mid *$

Ungarische Aipen $|*| *|*| *$

Kroatische Alper

Für das ganze

Staatsgebiet | $597,605719|\mathbf{1 2 6 0}| 1560$

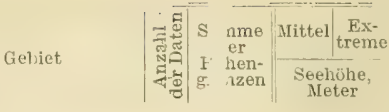

f) Agnrabisches Vorkommen im fichlentestand. where irenze

Max.

\begin{tabular}{l|l|l|l|l|l} 
Nordwestkarpathen. & 15 & 17711 & $\mathbf{1 1 8 0}$ & 1265
\end{tabular}

Centralkarpathen. . $|230| \quad 280391 \quad|\mathbf{1 2 6 0}| 1442$

\begin{tabular}{|l|l|l|l|l} 
Nordostkarpathen . & 227 & 291520 & $\mathbf{1 2 8 0}$ & 1446
\end{tabular}

\begin{tabular}{|l|l|l|l|l|}
\hline Ostkarpathen $\ldots$ & 118 & 157554 & $\mathbf{1 3 4 0}$ & 15.18
\end{tabular}

Sülkarpathen *. $\quad 142|196148| \mathbf{1 3 8 0} \mid 1600$

Sïdung. Gebirgsland | $42|\quad 58928 \quad 1400| 1560$

\begin{tabular}{c|c|c|c|c|} 
Bihargebirge. . . . & 74 & 9050 & $\mathbf{1 3 5 0}$ & 1491
\end{tabular}

Ung. Mittelgebirge . | * | *

Inselgebirge ..... $\left.\right|_{*} \mid$

Ungarische Alpen . $|*| *|* *| *$

\begin{tabular}{ll|l|l|l|l} 
Kroatische Alpen : & 4 & 5566 & $\mathbf{1 3 9 0}$ & 1563 \\
\hline
\end{tabular}

Für das ganze

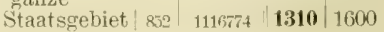

g) Strauchform, obeve firenze

\begin{tabular}{|l|l|l||l|l|l|l|} 
Centralkarpathen. . & 119 & 150899 & $\mathbf{1 3 5 0}$ & 1481
\end{tabular}

\begin{tabular}{l|l|l|l|l} 
Nordostkarpathen $\cdot \mid 129$ & 172210 & $\mathbf{1 3 4 0}$ & 1458
\end{tabular}

\begin{tabular}{ll|l|l|l|} 
Ostkarpathen $\cdots$ & 39 & 39085 & $\mathbf{1 4 0 0}$ & 1551
\end{tabular}

Südkarpathen . . . $22|31712 \quad| 1440 \mid 157 \%$

\begin{tabular}{l|l|l|l|l} 
Sitdung. Gebirgsland & 96 & 37548 & $\mathbf{1 4 4 0}$ & 1562
\end{tabular}

\begin{tabular}{|l|l|l||l|l|} 
Bihargebirge . . . . & 4 & 5989 & $\mathbf{1 5 0 0}$ & 1581
\end{tabular}

Ung. Mittelgebirge . * $\mid$ * || * | *

Inselgebirge .... * * $\mid$ * * $\quad * \quad * \quad *$

Ungarische Alpen . *

\begin{tabular}{|l|l|l|l|l|}
\hline Kroatische Alpen . & 37 & 55936 & $\mathbf{1 5 1 0}$ & 1699
\end{tabular}

\begin{tabular}{|l|l|l|l|l} 
Für das ganze & & & & \\
Staatsgebiet & 373 & 513110 & $\mathbf{1 3 8 0}$ & 1699
\end{tabular} 


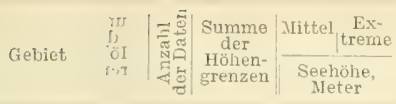

\section{Quereus sessiliflora Salisb.}

a) Bestandbildend, obere firenze Vax. \begin{tabular}{l|l|l|l|l|l|} 
Nordwestkarpathen. & 9 & 4853 & $\mathbf{5 1 0}$ & $\mathbf{6 4 0}$
\end{tabular}

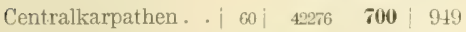

\begin{tabular}{l|l|l|l|l} 
Nordostkarpathen . & 121 & 81110 & $\mathbf{6 7 0}$ & 957
\end{tabular}

Ostkarpathen ... $|45| 37365: 8 \mathbf{8 3 0} \mid 1075$

\begin{tabular}{ll|l|ll|l|l} 
Süidkarpathen... & 16 & 15883 & $\mathbf{9 6 0}$ & 1058
\end{tabular}

\begin{tabular}{l|l|l|l|l} 
Sïdung. Gebirgsland & 99 & Gs8r1 & $\mathbf{7 0 0}$ & 890
\end{tabular}

Bihargebirge. . . $|36|$ צnc04 $|\mathbf{7 7 0}| \mathbf{1 0 4 4}$

Ung. Mittelgebirge . $|52| 3434|\mathbf{6 6 0}| 899$

Inselgebirge . . . $|30| 14090, \mathbf{4 7 0} \mid 815$

\begin{tabular}{|l|l|l|l|l|} 
Ungarische Alpen . & 5 & 2817 & 660 & 739
\end{tabular}

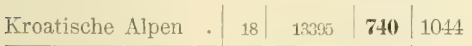

Für das ganze \begin{tabular}{l|l|l|l|l|} 
Stantsgebiet & 491 & 34099 & 700 & 1075
\end{tabular}

13) Vereinzelt, ohere firenze

Miax.

\begin{tabular}{l|l|ll|l} 
Nordwestkarpathen. & 21 & 14321 & $\mathbf{6 8 0}$ & 802
\end{tabular}

Centralkarpathen . - 139 114:-; 800 1145

\begin{tabular}{l|l|ll|l} 
Noriostkarpathen $|123|$ & 93774 & $\mathbf{7 6 0}$ & 1112
\end{tabular}

\begin{tabular}{ll|l|l|l|l} 
Ostkarpathen , . & 52 & 48911 & $\mathbf{9 4 0}$ & $\mathbf{1 1 2 0}$
\end{tabular}

Südkarpathen . . - $|23| 23825|990| 1217$

\begin{tabular}{l|l|l|l|l|} 
Süidung. Gebirgsland $\mid$ & $102 \mid$ & 74570 & $\mathbf{7 3 0}$ & 992
\end{tabular}

Bihargebirge... . | 60| $51+83|860| 1106$

\begin{tabular}{l|l|ll|l} 
Ung. Mittelgebirge . & $44 \mid 29002$ & $\mathbf{6 0 0}$ & 939
\end{tabular}

\begin{tabular}{l|l|l|l|l} 
Inselgebirge. . . . & 96 & 13918 & 540 & 815
\end{tabular}

\begin{tabular}{l|l|l|l|l} 
Ungarische Alpen . & 3 & $\mathbf{1 7 4 1}$ & $\mathbf{8 7 0}$ & 883
\end{tabular}

\begin{tabular}{ll|l|l|l} 
Kroatische Alpen . & 23 & $\mathbf{1 8 0 4 2}$ & $\mathbf{7 9 0}$ & 1044
\end{tabular}

Für das ganze

Staatsgebiet | $615 \quad 470310 \quad \mathbf{7 8 0} \quad 1217$

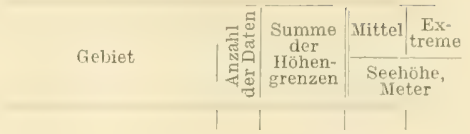

c) Verkrïppelt, ohere trenze

Max.

Nordwestlearpathen $\mid+1$

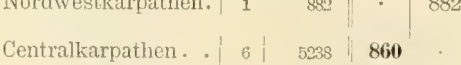

\begin{tabular}{|l|l|l|l|l|} 
Nordostkarpathen . & 9 & 9515 & $\mathbf{1 0 6 0}$ & 1201
\end{tabular}

\begin{tabular}{l|l|l|l|l} 
Ostkarpathen &. & 13 & 13507 & $\mathbf{1 0 4 0}, 1336$
\end{tabular}

Sïikarpathen . . . .

\begin{tabular}{l|l|l|l|l} 
Süidung. Gebirgsland & 8 & 6937 & 870 & 1020
\end{tabular}

Bihargebirge. . . . . ;

\begin{tabular}{l|l|l|l|l} 
Ung. Mittelgebirge .. & 1 & 940 & . & 940
\end{tabular}

Inselgebirge .....

Ungarische Alpen

Kroatische Alpen

Für das ganze

\begin{tabular}{l|l|l} 
Staatsgebiet & 38 & 38019
\end{tabular}

9701336

(quercus pedunculata Ehrli. (Qu. Robur L.)

Obere Grenze Max.

Nordwestkarpathen. | $28 \mid 12702$, $450 \mid 618$

Centralkarpathen. . | 29, $18752 \mid \mathbf{6 5 0}$ ! 951

Nordostkarpathen . $\mid$ 60 $\mid 41288$ | 690 1088

\begin{tabular}{l|l|l|l|l} 
Ostkarpathen . . . & 34 & 26211 & $\mathbf{7 7 0}$ & 993
\end{tabular}

Südkarpathen ... | $15|\operatorname{9191}| \mathbf{6 1 0} \mid 686$

\begin{tabular}{l|l|l|l|l} 
Südung. Gebirgsland & 6 & 3520 & $\mathbf{5 9 0}$ & $\mathbf{7 6 6}$
\end{tabular}

Bihargebirge ... $17 \mid$\begin{tabular}{l|l|l}
12318 & 730 & 960
\end{tabular}

\begin{tabular}{l|l|l|l|l} 
Ung. Mittelgebirge & 26 & 10768 & $\mathbf{4 1 0}$ & 731
\end{tabular}

\begin{tabular}{l|l|l|l|l|l} 
Inselgebirge . . . & 2 & 789 & $\mathbf{4 0 0}$ & 401
\end{tabular}

\begin{tabular}{l|l|l|l|l} 
Ungarische Alpen . & 1 & 543 & $\cdot$ & 543
\end{tabular}

Kroatische Alpen . $|19| 10736 \quad \mathbf{5 7 0} \quad 709$

\begin{tabular}{|l|l|l|l|l|l|} 
Erclélyer Becken. . & 1 & 685 & $\cdot$ & 685
\end{tabular}

Für das ganze

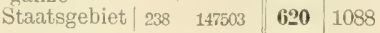




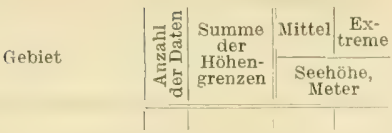

\section{Qquercus lanuginosa Lam.}

Obere Grenze

Nordwestkarpathen. | |

\begin{tabular}{ll|l|l|l|l|} 
Centralkarpathen. & 3 & 1615 & $\mathbf{5 4 0}$ & 609
\end{tabular}

\begin{tabular}{|l|l|l|l|l} 
Nordostkarpathen . & 1 & 400 & . & 400
\end{tabular}

Ostkarpathen ...

Südkarpathen

Südung, Gebirgsland 1

Bihargebirge .... 3

Ung. Mittelgebirge . 31

Inselgebirge . . 10 .

Ungarische Alpen . |

Kroatische Alpen • 15

Erdélyer Becken . . 1

Für das ganze

Staatsgebiet 65

38276

590

\section{Quercus conferta Kit.}

a) Bestandblldend, obere Grenze

Nordwestkarpathen. $|-|-|+|-$

Centralkarpathen. . | $-1-1-1-$

Nordostkarpathen . $\mid-1-1-1-$

\begin{tabular}{ll|l|l|l} 
Ostkarpathen ... & - & - & - \\
\hline
\end{tabular}

\begin{tabular}{|l|l|l|l|l|l|} 
Südkarpathen . . . & 1 & 446 & - & 446
\end{tabular}

Südung. Gebirgsland $\mid$\begin{tabular}{l|l|l|l}
15 & 6411 & $\mathbf{4 3 0}$ & 592
\end{tabular}

Bihargebirge. . . $\mid$\begin{tabular}{l|l|l|l}
8 & 3131 & $\mathbf{3 9 0}$ & 450
\end{tabular}

Ung. Mittelgebirge . $|-|-1-1-$

Inselgebirge . . . . . . .

Ungarische Alpen . $|-|-1-1-$

Kroatische Alpren

Für das ganze enet 24
Gebiet

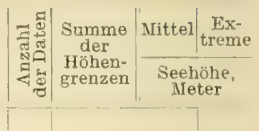

b) Vereinzelt, obere Grenze

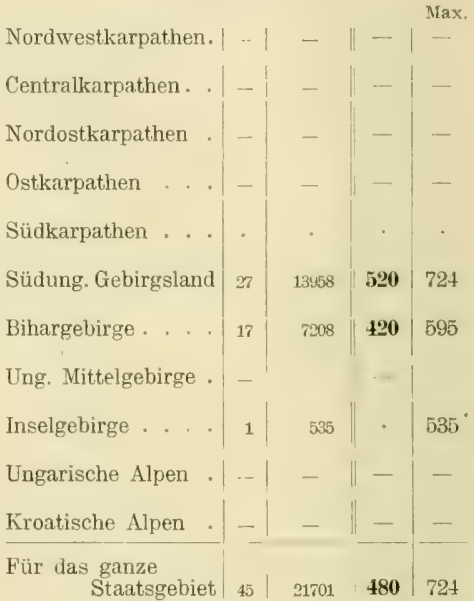

Quereus Cerris L.

obere Grenze

Max.

\begin{tabular}{lll|l|l} 
Nordwestkarpathen. & 1 & 430 & $\cdot$ &
\end{tabular}

\begin{tabular}{l|l|l|l|l} 
Centralkarpathen. . & 35 & 25214 & $\mathbf{7 2 0}$ & 981
\end{tabular}

\begin{tabular}{l|l|l|l|l} 
Nordostkarpathen . & 1 & 589 & | . & 589
\end{tabular}

Ostkarpathen ....

\begin{tabular}{|l|l|l|l|l} 
Südkarpathen . . . & 2 & 1224 & $\mathbf{6 1 0}$ & 680
\end{tabular}

Südung, Gebirgsland $\quad 30 \quad 19266 \quad \| \mathbf{6 4 0} \quad 1018$

\begin{tabular}{|c|c|c||c|c|} 
Bihargebirge . . . & 13 & 8161 & $\mathbf{6 3 0}$ & 837
\end{tabular}

\begin{tabular}{l|l|l|l|l} 
Ung. Mittelgebirge . & 28 & 17971 & $\mathbf{6 4 0}$ & 945
\end{tabular}

\begin{tabular}{|l|l|l|l|l} 
Inselgebirge . . . & 9 & 4703 & $\mathbf{5 2 0}$ & 682
\end{tabular}

\begin{tabular}{l|l|l|l|l}
\hline Ungarische Alpen . & 1 & 755 & $\cdot$ & 755
\end{tabular}

\begin{tabular}{ll|lll} 
Kroatische Ilpen . & 17 & 1364 & 800 & 487
\end{tabular}

Für das ganze 


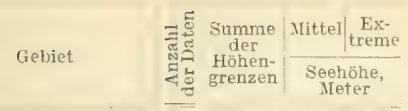

\section{Carpinus Betulus L.}

a) Bestandbildend, obere tiren $\iota$ e

\begin{tabular}{l|l|l} 
Nordwestkarpathen. & 26 & 10993
\end{tabular}

\begin{tabular}{l|l|l} 
Centralkarpathen. . & 50 & 12729
\end{tabular}

Nordostkarpathen .| $9 \mid 4922$

\begin{tabular}{l|l|l|l|} 
Ostkarpathen $\ldots$ & 3 & 34
\end{tabular}

Südkarpathen ... .

Südung. Gebirgsland .

\begin{tabular}{l|l|l} 
Bihargebirge . . . & 7 & 7519
\end{tabular}

\begin{tabular}{l|l|l} 
Ung. Mittelgebirge . & 12 & 7618
\end{tabular}

Inselgebirge .... $|9| 4282$

Ungarische Alpen . .

\begin{tabular}{l|l|l|} 
Kroatische Alpen : & 18 & 13079 \\
\hline
\end{tabular}

Für das ganze

Staatsgebiet $104 \mid 61566$

b) Tereinzelt, obere irrenze

\begin{tabular}{|c|c|c|c|c|}
\hline Nordwestkarpathen. & 15 & 9423 & 630 & 867 \\
\hline Centralkarpathen. . & 118 & 85921 & 730 & 1017 \\
\hline Nordostkarpathen . & 110 & 94824 & 680 & 949 \\
\hline Ostkarpathen ... & 57 & $197 \pm 9$ & 870 & 1172 \\
\hline Südkarpathen . . . & 40 & $3 \overline{7} 723$ & 890 & 1188 \\
\hline Siidnug. trebiruslant & 33 & $f_{2}^{\prime 2}+11$ & 800 & $111 \varnothing$ \\
\hline Bihargebirge... & 88 & $73 \overline{5057}$ & 840 & 1115 \\
\hline Ung. Mittelgebirge . & 21 & 17226 & 820 & 955 \\
\hline Inselgebirge .... & 12 & 6336 & 530 & 953 \\
\hline Ungarische Alpen . & 1 & 739 & . & 739 \\
\hline Kroatische Alpen . & 25 & 18995 & 760 & 1035 \\
\hline $\begin{array}{l}\text { Für das ganze } \\
\text { Staatsgebiet }\end{array}$ & 580 & 434754 & 760 & 118 \\
\hline
\end{tabular}

Max. $\mathbf{4} \mathbf{0} \mid 562$

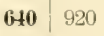

500

$810 \mid 920$

$790 \quad 859$

$640 \mid 895$

480682

730

900

590

920

Ciebiet

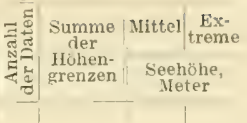

c) Strauchform, obere firenze

Nordwestkarpathen.

Centralkarpathen.

\begin{tabular}{l|l|l} 
Nordostkarpathen. & 47 & 41264
\end{tabular}

\begin{tabular}{|l|l|l|} 
Ostkarpathen . . . & 4 & 3836
\end{tabular}

Süidkarpathen . . 4

Südung. Gebirgsland 6 6150

\begin{tabular}{|l|l|l} 
Bihargebirge . . . . & 2 & 6010
\end{tabular}

\begin{tabular}{l|l|l} 
Ung. Mittelgebirge . & 4 & 3685
\end{tabular}

Inselgebirge

Ungarische Alpen

\begin{tabular}{l|l|l|l|l} 
Kroatische Alpen & 4 & 3832 & $\mathbf{9 6 0}$ & 1084 \\
\cline { 1 - 3 } Für das ganze & & & & \\
Staatsgebiet & 75 & 69108 & $\mathbf{9 2 0}$ & 1294
\end{tabular}

\section{Carpinus duinensis Scop.}

obere Grenze

Max.

\begin{tabular}{|c|c|c|c|c|}
\hline & & & & \\
\hline Nordwestkarpathen. & - & - & - & - \\
\hline Centralkarpathen. . & - & - & - & - \\
\hline Nordostkarpathen . & - & - & - & - \\
\hline Ostkarpathen ... & - & - & - & - \\
\hline Südkarpathen . . . & - & - & - & \\
\hline Sïdung. Gebircrsland & 24 & 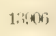 & 580 & 1046 \\
\hline Bihargebirge . . & - & - & - & - \\
\hline Ung. Mittelgebirge . & - & - & 一 & - \\
\hline Inselgebirge . . . . & 1 & 300 & . & 200 \\
\hline Ungarische Alpen & - & & & \\
\hline Kroatische Alpen . & 17 & 11434 & 670 & 1104 \\
\hline $\begin{array}{l}\text { Für das ganze } \\
\text { Staatsgebiet }\end{array}$ & 42 & $255+0$ & 610 & 110 \\
\hline
\end{tabular}




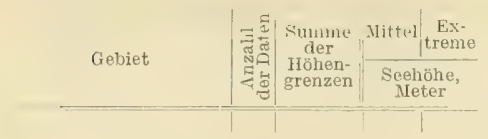

\section{Corylus Arellana L.}

Obere Grenze

Nordwestkarpathen. $|\cdot 6| \quad 5163$

\begin{tabular}{|l|l|l|l|l|l|} 
Centralkarnathen. & 55 & 55583 & $\mathbf{1 0 5 0}$ & 1341
\end{tabular}

\begin{tabular}{l|c|c|c|c|c|} 
Nordostkarpathen . & 163 & 158370 & $\mathbf{9 7 0}$ & 1343
\end{tabular}

\begin{tabular}{|l|l|l|l|l|l|} 
Ostkarpathen $\ldots$ & $\mathbf{1 1 6 0}$ & 1528
\end{tabular}

Südkarpathen ... $52|59524| \mathbf{1 1 5 0} \mid 1412$

\begin{tabular}{l|l|l|l|l|} 
Südung. Gebirgsland & $10 \mid$ & 11199 & $\mathbf{1 1 2 0}$ & 1328
\end{tabular}

\begin{tabular}{|l|l|l|l|l|} 
Bihargebirge . . . . 45 & 50713 & $\mathbf{1 1 3 0}$ & 1432 \\
\hline
\end{tabular}

\begin{tabular}{l|l|l|l} 
Ung. Mittelgebirge . & 1 & 1005
\end{tabular}$\cdot 1005$

Inselgebirge .... * * * * * $*$

Ungarische Alpen . $*$ * $\mid$ * $\mid *$ *

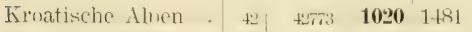

Für das ganze

\begin{tabular}{l|l|l|l|l} 
Staatsgebiet & 438 & 460594 & $\mathbf{1 0 5 0}$ & 1528
\end{tabular}

Betula verrucosa Elur.

obere firenze

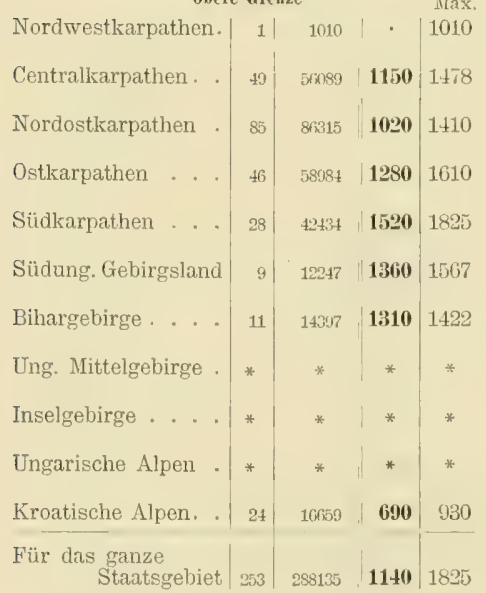

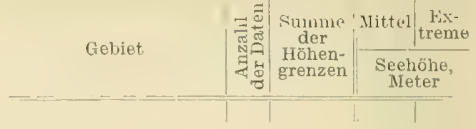

Alums griutinosa Gairtn.

obere Grenze

M[ax.

\begin{tabular}{ll|l|l|l} 
Nordwestkarpathen. $\mid$ & 8 & 4691 & $\mathbf{5 9 0}$ & 688
\end{tabular}

\begin{tabular}{ll|l||l|l|} 
Centralkarpathen. . & 63 & 49032 & $\mathbf{7 8 0}$ & 1290
\end{tabular}

\begin{tabular}{l|l||l|l|l} 
Nordostkarpathen . & 97 & 18552 & $\mathbf{6 9 0}$ & 1020
\end{tabular}

\begin{tabular}{ll|l||l|l|} 
Ostkarpathen $\cdots$ & 33 & 28039 & $\mathbf{8 5 0}$ & $11: 20$
\end{tabular}

\begin{tabular}{|l|l|l||l|l} 
Südkarpathen . . . & 30 & 24313 & $\mathbf{8 1 0}$ & 1250
\end{tabular}

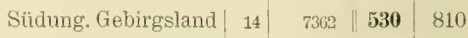

\begin{tabular}{|l|l|l||l|l|} 
Bihargebirge. . . . 21 & 12397 & $\mathbf{5 9 0 0}$ & 950
\end{tabular}

\begin{tabular}{l|l||l|l|l} 
Ung. Mittelgebirge . & 5 & 3594 & $\mathbf{7 2 0}$ & 873
\end{tabular}

Inselgebirge . . . $1000 \quad 1 \quad \cdot 600$

\begin{tabular}{l|l|l|l|l} 
Ungarische Alpen . & 1 & 518 & & 518
\end{tabular}

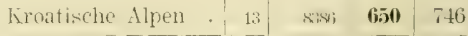

Für das ganze

\begin{tabular}{|l|l|l|l|l} 
Staatsgebiet & 216 & 157484 & $\mathbf{7 3 0}$ & 1290
\end{tabular}

\section{Alnus incana Willd.}

\section{Obere Grenze}

Max.

\begin{tabular}{ll|l|l|l} 
Nordwestkarpathen. | & 7 & 4995 & $\mathbf{7 1 0}$ & $\mathbf{7 1 0}$
\end{tabular}

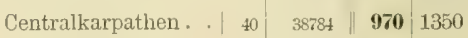

\begin{tabular}{l|l|l|l|l} 
Nordostkarpathen . & 67 & 62297 & $\mathbf{9 3 0}$ & 1281
\end{tabular}

\begin{tabular}{|l|l|l|l|l} 
Ostkarpathen $\ldots$ & 20 & 21887 & 1090 & 1303
\end{tabular}

\begin{tabular}{|l|l||l|l|l} 
Südkarpathen . . . & 40 & 34941 & $\mathbf{8 7 0}$ & 1284
\end{tabular}

\begin{tabular}{ll|l|l|l} 
Stidung. Gebirgsland & 9 & 7177 & $\mathbf{8 0 0}$ & 951
\end{tabular}

\begin{tabular}{|l|l|l|l|l} 
Bihargebirge. . . . & 19 & 17611 & $\mathbf{9 3 0}$ & 1145
\end{tabular}

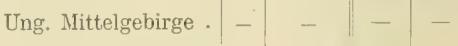

Inselgebirge .... | - - $-1-1-$

\begin{tabular}{l|l|l|l|l} 
Ungarische Alpen * & 1 & 322 & $\cdot$ & 322
\end{tabular}

\begin{tabular}{|l|l|l|l} 
Kroatische Alpen . | $3 \mid$ & 3367 & $\mathbf{7 9 0}$ & 878
\end{tabular}

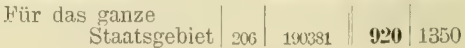




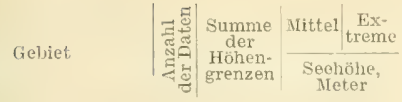

\section{Alus viridis bC.}

a) Untere frenze

Nordwestkarpathen. $|-|-$

Centralkarpathen .

\begin{tabular}{l|l|ll|l} 
Nordostkarpathen & 109 & $110 y 18$ & $\mathbf{1 0 2 0}$ & $40 \mathrm{~s}$
\end{tabular}

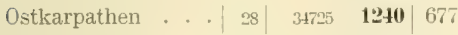

\begin{tabular}{|l|l|ll|l} 
Südkarpathen . . . & 13 & 12394 & $\mathbf{9 5 0}$ & 660
\end{tabular}

\begin{tabular}{l|l|l|l|l} 
Südung. Gebirgsland & 2 & 1904 & $\mathbf{9 5 0}$ & 680
\end{tabular}

\begin{tabular}{|l|l|ll|l} 
Bihargebirge.... & 4 & 5582 & $\mathbf{1 4 0 0}$ & 937
\end{tabular}

Ung. Mittelgebirge . $|-|-|+|-$

Inselgebirge . . . $|-|-1-1-$

Ungarische Alpen . | $9|\quad 3597, \mathbf{1 0 0}| 319$

\begin{tabular}{l|l|ll|l} 
Kroatische Alpen & - & - & - & - \\
Für das ganze & & & & \\
Staatsgebiet & 160 & 169120 & $\mathbf{1 0 2 0}$ & 319
\end{tabular}

b) there tirenze

Max.

Nordwestkarpathen. $-1-1-1-$

Centralkarpathen. . $|-|-\quad-1-$

\begin{tabular}{l|l|l|l|l} 
Nordostkarpathen & 94 & $1516 r 0$ & $\mathbf{1 6 1 0}$ & 2000
\end{tabular}

\begin{tabular}{ll|l|l|l|l|} 
Ostkarpathen... $\mid$ & 16 & 29123 & $\mathbf{1 8} \geq 0$ & 2001
\end{tabular}

\begin{tabular}{ll|l|ll|l|l} 
Sitflkarpathen... & 34 & 65405 & $\mathbf{1 9} 20$ & 2121
\end{tabular}

\begin{tabular}{l|l|l|l|l} 
Sïdung. Gebirgsland & 7 & 11723 & $\mathbf{1 6 8 0}$ & 1960
\end{tabular}

\begin{tabular}{ll|l|l|l|l|l|} 
Bihargebirge. . . & 3 & 5 & $\mathbf{1 7 4 0}$ & 1780
\end{tabular}

Ung. Mittelgebirge . $|-|-1-1-$

Inselgebirge $\ldots .+-\mid-1-1-$

Ungarische Alpen . $|*|$ * $\quad *$ * $\mid$ *

Kroatische Alpen : $|-|=\mid-1-$

Für das ganze

$$
\begin{array}{l|l|l|l|l|}
\text { Staatsgebiet } & 154 & 361134 & \mathbf{1 7 0 0} & 2121
\end{array}
$$

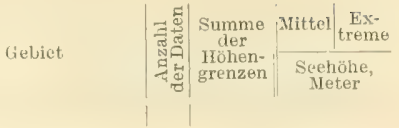

Salix alba $\mathrm{L}$.

Obere Girenze Max

\begin{tabular}{l|l|l|l|l|} 
Nordwestkarpathen. & 1 & 8 & & 823
\end{tabular}

\begin{tabular}{l|l|l|l|l} 
Centralkarpathen. . & 1 & 813 & $\cdot$ & 813
\end{tabular}

\begin{tabular}{l|l|l|l|l} 
Nordostkarpathen . & 1 & 1058 & $\cdot$ & 1058
\end{tabular}

\begin{tabular}{|l|l|l|l|l} 
Ostkarpathen . . . & 1 & 998 & & 998
\end{tabular}

\begin{tabular}{|l|l|l|l|l} 
Sülkarpathen .... & 2 & 1464 & $\mathbf{7 3 0}$ & 780
\end{tabular}

\begin{tabular}{l|l|l|l|l} 
Südung. Gebirgsland & 5 & 3687 & $\mathbf{7 4 0}$ & $\mathbf{1 2 5 0}$
\end{tabular}

\begin{tabular}{|l|l|l||l|l|} 
Bihargebirge . . . & 26 & 2476 & $\mathbf{9 4 0}$ & $\mathbf{1 2 0 0}$
\end{tabular}

\begin{tabular}{l|l|l|l|l} 
Ung. Mittelgebirge . & 1 & 608 & $\cdot$ & 608
\end{tabular}

\begin{tabular}{l|l|l|l|l} 
Inselgebirge . . . . & 1 & 380 & $\cdots$ & 380
\end{tabular}

Ungarische Alpen .| 1 | $3 * 2, \cdot$ | 322

\begin{tabular}{l|l|l|l|l} 
Kroatische Alpen : & 12 & 8455 & $\mathbf{7 1 0}$ & 941 \\
\hline & &
\end{tabular}

Für das ganze

\begin{tabular}{l|l|l|l|l} 
Staatsgebiet & 52 & 43114 & $\mathbf{8 3 0}$ & $\mathbf{1 2 5 0}$
\end{tabular}

\section{populus tremula L.}

a) Baumform, olsere tirenze Max.

\begin{tabular}{l|l|l|l|l} 
Nordwestkarpathen. & 7 & 5305 & $\mathbf{7 5 0}$ & 922
\end{tabular}

\begin{tabular}{l|l|l|l|l|l|} 
Centralkarpathen. . & 46 & 50877 & $\mathbf{1 1 1 0}$ & 1425
\end{tabular}

\begin{tabular}{|l|l|l||l|l|} 
Nordostkarpathen * & 121 & 105501 & $\mathbf{8 8 0}$ & 1204
\end{tabular}

\begin{tabular}{ll|l|l|l|} 
Ostkarpathen . . . | 27 & 31887 & $\mathbf{1 1 8 0}$ & 1397
\end{tabular}

\begin{tabular}{|l|l|l|l|l|l|} 
Südkarpathen . . . & 28 & 33255 & $\mathbf{1 1 9 0}$ & 1593
\end{tabular}

\begin{tabular}{l|l|l|l|l} 
Sidung. Gebirgsland & 0 & 6906 & $\mathbf{1 1 5 0}$ & 1486
\end{tabular}

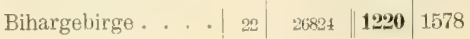

Ung. Mittelgebirge . $*$ * * * || * *

Inselgebirge .... * * ${ }^{*} * *^{*} \mid *$

\begin{tabular}{l|l|l|l|l} 
Ungarische Alpen . & $*$ & $*$ & $*$
\end{tabular}

\begin{tabular}{l|l|l|l|l} 
Kroatische Alpen & 22 & 19166 & 870 & 1151
\end{tabular}

\begin{tabular}{l|l|l|l|l} 
Staatsgebiet & 279 & 280171 & $\mathbf{1 0 0 0}$ & 1593
\end{tabular} 


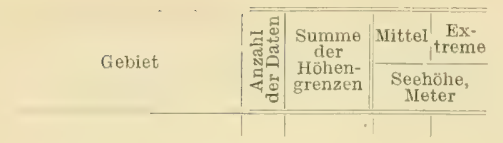

b) Strauchform, obere Grenze

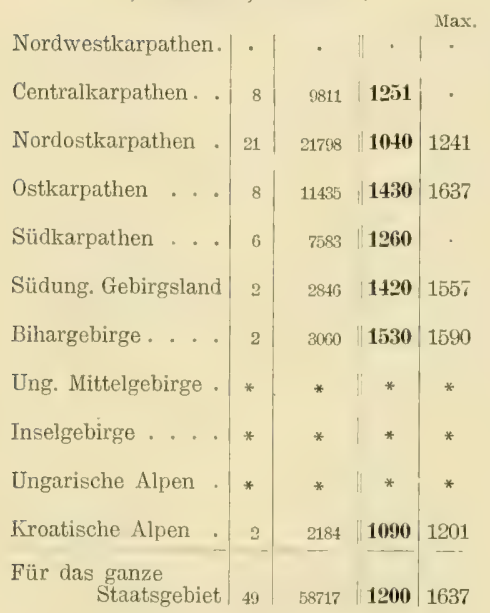

Ulmus montana With. (U. scabra Mill.)

\begin{tabular}{|c|c|c|c|c|}
\hline \multicolumn{4}{|c|}{ a) Untere (irenze } & Min. \\
\hline Nordwestkarpathen. & 3 & 750 & 250 & 236 \\
\hline Centralkarpathen. . & 11 & 3861 & 350 & 280 \\
\hline Nordostkarpathen & 35 & 12374 & 350 & 179 \\
\hline Ostkarpathen . . & 15 & 10753 & 720 & 478 \\
\hline Südkarpathen . . & 5 & 3663 & 530 & 375 \\
\hline Südung. Gebirgsland & 9 & 3868 & 430 & 345 \\
\hline Bihargebirge . . . & 7 & 2220 & 320 & 221 \\
\hline Ung. Mittelgebirge & 5 & 1422 & 280 & 219 \\
\hline Inselgebirge . . & 1 | & 494 & . & 49.1 \\
\hline Ungarische Alpen & . & . & . & \\
\hline Kroatische Alpen . & 9 & 4209 & 470 & 190 \\
\hline Erdélyer Becken. . & 1 & 348 & - & 348 \\
\hline $\begin{array}{l}\text { Für das ganze } \\
\text { Staatsgebiet }\end{array}$ & 101 & 42962 & 430 & 179 \\
\hline
\end{tabular}

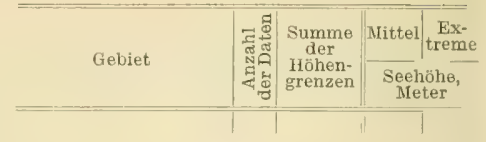

b) Obere Grenze

\begin{tabular}{|c|c|c|c|c|}
\hline Nordwestkarpathen. & 9 & 6766 & 750 & $\begin{array}{r}\text { Max. } \\
962\end{array}$ \\
\hline Centralkarpathen. . & 93 & 96683 & 1040 & 1280 \\
\hline Nordostkarpathen . & 165 & 171044 & $10 \pm 0$ & 1365 \\
\hline Ostkarpathen ... & 59 & 70007 & 1190 & 1410 \\
\hline Südkarpathen . . . & 48 & 52220 & 1090 & 1430 \\
\hline Südung. Gebirgsland & 35 & 38060 & 1090 & 1460 \\
\hline Bihargebirge . . . . & 38 & 35737 & $9 \pm 0$ & 1262 \\
\hline Ung. Mittelgebirge . & * & * & * & * \\
\hline Inselgebirge . . . . & * & * & * & * \\
\hline Ungarische Alpen . & * & * & * & * \\
\hline Kroatische Alpen & 19 & 20256 & 1070 & 1350 \\
\hline Erdélyer Becken. . & * & * & * & $\pi$ \\
\hline ir das gar & 466 & 490773 & 1050 & 146 \\
\hline
\end{tabular}

\section{Ulmus campestris L. (U. glabra Mill.)}

obere Grenze Max.

Nordwestkarpathen | $1|485||\cdot| 485$

\begin{tabular}{l|l|l|l|l|l} 
Centralkarpathen. . & 7 & 4582 & $\mathbf{6 6 0}$ & 892
\end{tabular}

Nordostkarpathen . . . . . . . . .

\begin{tabular}{l|l|l|l|l} 
Ostkarpathen . . . & 1 & 708 & $\cdot$ & 708
\end{tabular}

\begin{tabular}{l|l|l|l|l|l|} 
Südkarpathen . . . & 1 & 878 &. & 878
\end{tabular}

\begin{tabular}{l|l|l|l|l} 
Südung. Gebirgsland & 1 & 970 & $\cdot$ & 970
\end{tabular}

\begin{tabular}{|l|l|l|l|l|} 
Bihargebirge . . . & 1 & 828 & & \\
\hline
\end{tabular}

Ung. Mittelgebirge .

Inselgebirge ... $11435 \| \cdot \mid 435$

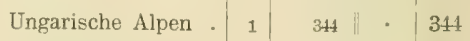

Kroatische Alpen * 1 1690 || * 690

\begin{tabular}{|l|l|l|l|l|l|} 
Erdélyer Becken. . & 6 & 3378 & $\mathbf{5 6 0}$ & 676
\end{tabular}

\begin{tabular}{l|l|l|l|l} 
Für das ganze & & & & \\
Staatsgebiet & 21 & 13298 & $\mathbf{6 3 0}$ & 970
\end{tabular} 


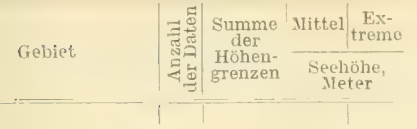

Tilia parvifolia Ehrh. (T. cordata Mill.)

\begin{tabular}{|c|c|c|c|c|c|}
\hline \multicolumn{5}{|c|}{ Obere Grenze } & \multirow{2}{*}{$\begin{array}{l}\text { Max. } \\
712\end{array}$} \\
\hline Nordwestkarpathen & & $4 \mid$ & 2783 & 700 & \\
\hline Centralkarpathen. & .1 & 15 & 13967 & 930 & 1130 \\
\hline Nordostkarpathen & . & 7. & 6427 & 920 & 1150 \\
\hline Ostkarpathen . . & & 17 & 15397 & 910 & 1308 \\
\hline Südkarpathen . . & . & 15 & 13517 & 900 & 1188 \\
\hline Südung. Gebirgslan & & 11 & 9994 & 910 & 1121 \\
\hline Bihargebirge . . . & . & 19 & 14231 & 750 & 1080 \\
\hline Ung. Mittelgebirge & . & $*$ & * & * & \\
\hline Inselgebirge . . . & . & 1 & 593 & - & 593 \\
\hline Ungarische Alpen & . & 1 & 757 & - & \\
\hline Kroatische Alpen & . & 15 & 15941 & 1060 & $15:$ \\
\hline üi das ganze & & 105 & 93607 & 890 & \\
\hline
\end{tabular}

Tilia grandifolia Ehrh. (T. platyphylla scop.)

Obere Grenze Max.

\begin{tabular}{l|l|l|l|l|l|l} 
Nordwestkarpathen. & 1 & 570 & - & 570
\end{tabular}

\begin{tabular}{l|l|ll|l} 
Centralkarpathen. & 16 & 14123 & 880 & 1053
\end{tabular}

\begin{tabular}{l|l|ll|l} 
Nordostkarpathen . & 9 & 5810 & $\mathbf{5 8 0}$ & 872
\end{tabular}

Ostkarpathen

Siidkarpathen . . . . . |

Südung. Gebirgsland $|10| \quad 8874 \quad 890 \mid 1167$

Bihargebirge . . . $11|7012 \quad 640| 900$

Ung. Mittelgebirge . $\left|\begin{array}{lll}10 & 8668 & \mathbf{8 7 0}\end{array}\right| 363$

\begin{tabular}{l|l|l|l|l|} 
Inselgebirge . . . . & 1 & 494 & 494
\end{tabular}

\begin{tabular}{l|l|l|l|l} 
Ungarische Alpen . & 1 & 739 & 739 \\
Kroatische Alpen . & 1 & 850 & $\cdot$ & 850
\end{tabular}

Fïr das ganze Staatsgebiet $60 \mid 46570$

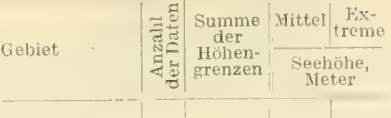

Tilia tomentosa Mönch.

\begin{tabular}{|c|c|c|c|c|}
\hline \multicolumn{4}{|c|}{ Obere Grenze } & Max. \\
\hline Nordwestkarpathen & $\mid-$ & - & - & - \\
\hline Centralkarpathen. . & - & - & - & \\
\hline Nordostkarpathen . & 1 & 512 & . & 512 \\
\hline Ostkarpathen ... & - & - & - & - \\
\hline Sizdkarpathen . . . & 1 & 693 & - & 692 \\
\hline Südung. Gebirgsland & 34 & 24098 & 710 & 916 \\
\hline Bihargebirge . . . . & 3 & 1880 & 630 & 715 \\
\hline Ung. Mittelgebirge . & - & - & - & - \\
\hline Inselgebirge . . . . & 1 & 492 & $\cdot$ & 492 \\
\hline Ungarische Alpen . & - & - & - & - \\
\hline Kroatische Alpen . & 1 & 900 & $\cdot$ & 900 \\
\hline Erdélyer Becken. . & 1 & 467 & $\cdot$ & 467 \\
\hline $\begin{array}{l}\text { ür das ganze } \\
\text { Staatsgeliet }\end{array}$ & $t^{2}$ & 29041 & 690 & 916 \\
\hline
\end{tabular}

Acer platanoides $L$.

a) Baumform, obere tirenze Max

\begin{tabular}{l|l|ll|l} 
Nordwestkarpathen. & 6 & 4920 & $\mathbf{8 2 0}$ & 878
\end{tabular}

\begin{tabular}{|c|c|c|c|c|} 
Centralkarpathen. . & 64 & 66847 & $\mathbf{1 0 4 0}$ & 1286
\end{tabular}

\begin{tabular}{ll|l|l|l|} 
Nordostkarpathen $\cdot 503$ & 210179 & $\mathbf{1 0 4 0}$ & 1279
\end{tabular}

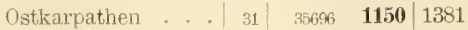

Siidkarpathen . . . $15 \mid \begin{array}{lll}15747 & \mathbf{1 0 5 0} & 1210\end{array}$

Siidung. Gebirgsland $|27| \quad 29116 \quad|\mathbf{1 0 8 0}| 1415$

\begin{tabular}{|l|l|l|l|l|} 
Bihargebirge. . . . & 37 & 36884 & $\mathbf{1 0 0 0}$ & 1250
\end{tabular}

Ung. Mittelgebirge : $|*| * \quad * \quad * \mid *$

Inselgebirge .... . $*|*| * *$ *

Ungarische Alpen .| * * * * *

Kroatische Alpen . $|14| \quad 13244 \quad 950 \mid 1100$

Fiir das ganze

Staatsgebiet $|387| 402633 \quad \mathbf{1 0 4 0} \mid 1415$ 
Giebiet

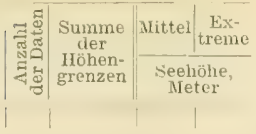

1) Strauchiorm. obera firenze

Jax.

Nordwestkarpathen. | . | . . . | .

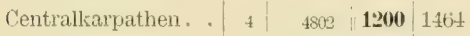

Nordostkarpathen , $|16| 1928, \mathbf{1 9 1 0} 1260$

Ostkarpathen

Südkarpathen

Südung, Gebirgsland

\begin{tabular}{|l|l|lll} 
Bihargebirge . . . & 2 & $25 \% 0$ & 1290 & 1330
\end{tabular}

Ung. Mittelgebirge . $|*|$ * * *

Inselgebilge .... $*$ * * * * * *

Ungarische Alpen . $|*|$ * * * * *

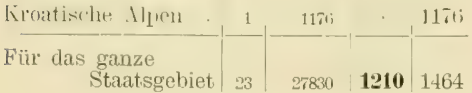

\section{Icer P'seudoplatanus L.}

a) IBumform, obere (drenze

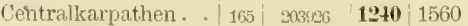

Nordostkarpathen . $|394| 491512$ 1250 1446

\begin{tabular}{|l|l|ll|l} 
Ostkarpathen $\ldots$ & 99 & 132968 & $\mathbf{1 3 4 0}$ & 1638
\end{tabular}

Süilkarpathen ... $80 \mid$\begin{tabular}{ll|l|l|}
114581 & $\mathbf{1 4 0 0}$ & 1652
\end{tabular}

Szidung. Gebirgsland $\mid$\begin{tabular}{ll|l|l|l|l|}
49 & 67193 & $\mathbf{1 3 7 0}$ & 1670
\end{tabular}

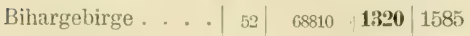

Ung. Mittelgebirge . $|*| *|+| *$

Inselgebirge .... $. * 1 * * * \mid *$

Ungarische Alpen .| * * * * * $\mid$ *

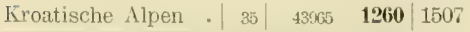

Für das ganze

\begin{tabular}{l|l|lll|l} 
Staatsgebiet & 879 & 1126404 & $\mathbf{1} 280$ & 1670
\end{tabular}

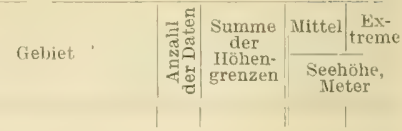

b) strunchform, ohere (irenze

Max.

Nordwestkarpathen. |

\begin{tabular}{|l|l|l|l|l|l|l|l|l|} 
Centralkarpathen. & 54 & 71341 & $\mathbf{1 3 2 0}$ & 1562
\end{tabular}

\begin{tabular}{l|l|l|l|l} 
Nordostkarpathen . & 51 & Gr663 & 1330 & 1564
\end{tabular}

\begin{tabular}{|l|l|l|l|l} 
Ostkarpathen $\cdots$ & 11 & 16606 & 1520 & 1725
\end{tabular}

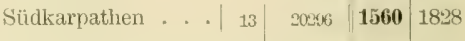

Südung, Gebirgsland .

Bihargebirge

Ung. Mittelgebirge $\cdot \mid *$

Inselgebirge

Ungarische Alpen . *

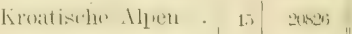

$1390 \div 1583$

Firr das ganze

$1370 \mid 1828$

Acer campestre L.

Gbere tirenze

$\mathrm{Iax}$

Nordwestkupathen. $\quad 5|3757,750| 777$

\begin{tabular}{|l|l|l|l|l|l} 
Centralkarpathen. & 67 & 4094 & $\mathbf{7 0 0}$ & 970
\end{tabular}

Nordostkarpathen . $|\mathbf{1 3}|$\begin{tabular}{r|r|r}
10490 & $\mathbf{8 1 0}$ & 909
\end{tabular}

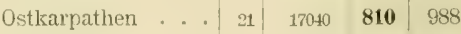

Südkarpathen . . . 10 $107097|\mathbf{7 1 0}| 1196$

Südung. Gebirgsland $22 \mid 16495 \quad$ 750 1104

Bihargebirge. . . . 33 | 35254 | $720 \mid 1090$

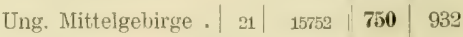

Inselgebirge ...| $1|\quad 815| \cdot \mid 815$

Ungarische Alpen .| .

\begin{tabular}{l|l|l} 
Kroatische Alpen - & 7 & 6587
\end{tabular}

$940 \quad 1148$

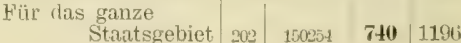




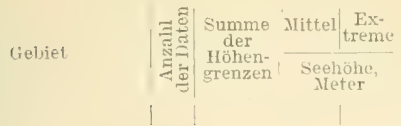

\section{Icer tataricum $\mathrm{L}$.}

\section{obere Grenze}

Nordwestkarpathen. .

\begin{tabular}{l|l|l} 
Centralkarpathen. . & 1 & 430
\end{tabular}

\begin{tabular}{l|l|l} 
Nordostkarpathen . & 1 & 333
\end{tabular}

Ostkarpathen . . . 1 427

Sïdkarpathen

Sïdung. Gebirgsland | 1 T45

Bihargebirge... . | 1 | 432

Ung. Mittelgebirge . 5 | 2595

Inselgebirge . . . | 1 | 361

Ungarische Alpen

Kroatische Alpen .|1 | 210

Erdélyer Becken . . | 1 | 534 . | 534

Füi das ganze

$$
\text { Staatsgebiet }\left|{ }_{13}\right| \text { fink7 } \quad \mathbf{4 7 0} \text { ! } 7 \text { t5 }
$$

Fraxinus excelsior $\mathbf{I}$.

Norwestkarpathen . | $17 \mid 13345$

\begin{tabular}{l|l|l} 
Centralkarpathen. . & $71 \mid 71890$
\end{tabular}

Nordostkarpathen . $|233| 235514$

Ostkarpathen ... | $36 \mid 38090$

Sïdkarpathen ... . $36 \mid 36689$

Siidung. Gebirgsland | $28 \mid 31499$

\begin{tabular}{l|l|l|} 
Bihargehirge . . . & $58 \mid 58570$
\end{tabular}

Ung. Mittelgebirge . $\mid$\begin{tabular}{l|l}
18 & 14512
\end{tabular}

Inselgebirge ... . | * | *

Ungarische Alpen $\cdot|*|$ *

Kioatische Alpen $\cdot|24| 2742$

Fiir das ganze

$$
\text { Staatsgebiet | } 521 \quad 527530
$$

$\operatorname{Max}$

$1010+1227$

1010 | 1300

$1060 \mid 1251$

$1020 \mid 1315$

$1130 \mid 1460$

$\mathbf{1 0 1 0} 1350$

$810 \mid 950$

where Grenze Max

Nordwestkarpathen. $|-|-\ldots-1-$

Centralkarpathen $\cdot|-|-$

Nordostkarpathen $\cdot 1-1-$

Ostkaxpathen

Sïilkarpathen

$117011 x i$

Sïdlung. Gebirgsland $: 27 \mid 19 \% 96$

$740 \mid 1103$

Bihargelirge . . . | $1 \mid 500$

500

\begin{tabular}{l|l|l} 
Ung. Mittelgebirge . & 21 & 11787
\end{tabular}

$560+808$

Inselgebirge .... $|4| 2183$

$5.50 \mid 612$

Ungarische Alpen

Kroatische Alpen . | $45 \mid 41059$

$910+1304$

Fïr das ganze

$$
\text { Stantsgebiet }|101| 70048 \quad 780 \mid 1304
$$

\section{I'irus communis l.}

0here fircues Nax.

Nordwestkarpathen. $\mid$ 1 $\mid$ \$70 | · | 970

\begin{tabular}{l|l|l|l|l|} 
Centrailkarpathen. . & 1 & $10 \times 3$
\end{tabular}

\begin{tabular}{l|l|l|l|l|l|l|} 
Nordostkarpathen . & 1 & 1179 & $\cdot$ & 1179
\end{tabular}

Ostkarpathen . . . $|1| 1128|| \cdot 1128$

Siiclkarpathen

Siidung. Gebirgsland | 1 | 9\&6 | .

Bihargebirge. . . . $|1| 111 \mid$. 1111

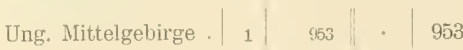

Inselgebirge . . . . $\mid$\begin{tabular}{l|l||}
1 & 688
\end{tabular}

Ungarische Alpen .

\begin{tabular}{|l|l|l|l|l} 
Kroatische Alpen . & 9 & 7648 & $\mathbf{8 5 0}$ & $105 \%$
\end{tabular}

Für dlas gauzu \begin{tabular}{l|l|l} 
Statsgebiet 17 & $15 \% 2$
\end{tabular}

$920+1179$ 


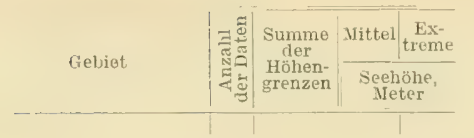

Pirus malus $\mathbf{L}$.

\begin{tabular}{|c|c|c|c|c|}
\hline \multicolumn{4}{|c|}{ Obere firenze } & Max. \\
\hline Nordwestkarpathen. & 1 & 722 & . & 722 \\
\hline Centralkarpathen. . & 7 & CH+12 & 920 & 1063 \\
\hline Nordostkarpathen . & 1 & 1035 & - & 1035 \\
\hline Ostkarpathen . . . & 5 & 5052 & 1010 & 1137 \\
\hline Südkarpathen . . . & 8 & 8656 & 1080 & 1208 \\
\hline Siidung. Gebirgsland & 1 & 774 & . & 764 \\
\hline Bihargebirge . . . . & 8 & 7966 & 1000 & 1132 \\
\hline Ung. Mittelgebirge . & 1 & 899 & - & 899 \\
\hline Inselgebirge . . . . & 1 & 562 & . & 562 \\
\hline Ungarische Alpen . & . & . & . & - \\
\hline Kroatische Alpen . & 8 & 7239 & 910 & $105 \%$ \\
\hline $\begin{array}{l}\text { Für das ganze } \\
\text { Staatsgebiet }\end{array}$ & 41 & 39347 & 960 & 1208 \\
\hline
\end{tabular}

Sorbus aucuparia $L$.

a) Baumform, obere cirenze

Nordwestkarpathen. $\quad 4 \mid \quad 4420$ !

Centralkarpathen. . $|104| 137104$

Nordostkaipathen $\cdot|137| 160056 \quad 1230 \mid 1496$

\begin{tabular}{ll|l|l|l|l} 
Ostkarpathen $\ldots$ & 57 & 78611 & $\mathbf{1 3 8 0}$ & 1676
\end{tabular}

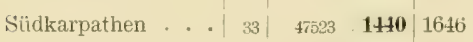

\begin{tabular}{l|l|l|l|l} 
Süidung. Gebirgsland & 9 & 12964 & $\mathbf{1 4 0}$ & 1753
\end{tabular}

\begin{tabular}{l|l|l|l|l} 
Bihargehirge. . . & 28 & 38202 & $\mathbf{1 3 6 0}$ & 1590
\end{tabular}

Ung, Mittelgebirge $|*| *|*| *$

Inselgebirge .. . $|*| *|*| *$

Ungarische Alpen . $|*| *|*| *|*| *$

\begin{tabular}{l|l|l|l|l} 
Kroatische Alpen & 7 & 7974 & $\mathbf{1 1 4 0}$ & 1410 \\
\hline Fize las ganze & & & & \\
Staatsgebiet & 379 & 490356 & $\mathbf{1 3 1 0}$ & 1753
\end{tabular}

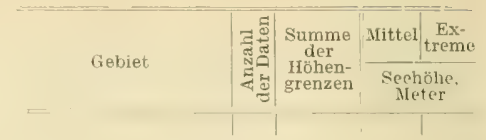

1) strunchform, obere (irens"

\begin{tabular}{|c|c|c|c|c|}
\hline Nordwestkarpathen. & 7 & 9813 & 1410 & $\begin{array}{r}\text { Max. } \\
1486\end{array}$ \\
\hline Centralkarpathen. . & 126 & 192855 & 1530 & 1777 \\
\hline Nordostkarpathen. & 88 & 124212 & 1410 & 1718 \\
\hline Ostkarpathen ... & 27 & 42756 & 1580 & 1841 \\
\hline Sïikarpathen . . . & 40 & 69041 & 1730 & 1987 \\
\hline Sïilung. Gebirgsland & 8 & 12156 & 1520 & 1851 \\
\hline Bihargebirge . . . & 4 & 5710 & 1430 & 1586 \\
\hline Ung. Mittelgebirge . & * & * & * & * \\
\hline Inselgebirge . . . . & * & * & * & * \\
\hline Ungarische Alpen . & * & * & * & * \\
\hline Kroatische Alpen. & 17 & 23728 & $\mid 1400$ & 1620 \\
\hline $\begin{array}{l}\text { iir das ganze } \\
\text { Staatsgehiet }\end{array}$ & 317 & 480301 & $15 * 0$ & \\
\hline
\end{tabular}

Sorbus torminalis Crantz.

obere Grenze

Nor.lwestkarpathen. $|\quad 4| \frac{2}{206}|\mathbf{6 6 0}| 748$

Centralkarpathen. . $|9|$\begin{tabular}{l|l|l|l|}
9 & 5958 & $\mathbf{6 6 0}$ & 800
\end{tabular}

\begin{tabular}{l|l|l|l|l} 
Nordostkarpathen . & 5 & 2174 & $\mathbf{4 0}$ & 519
\end{tabular}

\begin{tabular}{ll|l|l|l|l|} 
Ostkarpathen . . . & 1 & 878 & 878
\end{tabular}

\begin{tabular}{l|l|l|l|l} 
& & & & \\
Suidkarpathen . . . & 1 & 789 & - & 789
\end{tabular}

\begin{tabular}{l|l|l|l|l} 
Sülung. Gebirgsland & 19 & 11838 & $\mathbf{7 8 0}$ & 988
\end{tabular}

\begin{tabular}{l|l|l|l|l} 
Bihargebirge . . . & 4 & 2676 & $\mathbf{6 7 0}$ & 726
\end{tabular}

Ung. Mittelgebirge . $|12| 7305 \quad \mathbf{6 1 0} \mid 878$

Inselgebirge . . . 1 | $484|\cdots| 481$

\begin{tabular}{l|l|l|l|l} 
Ungarische Alpen . & 1 & 739 & $\cdot$ & 739
\end{tabular}

\begin{tabular}{l|l|l|l|l} 
Kroatische Alpen & 18 & 1214 & $\mathbf{6 7 0}$ & 950 \\
\hline
\end{tabular}

Fiur das ganze $\mid$\begin{tabular}{ll|l|l} 
& & & \\
& & &
\end{tabular} 


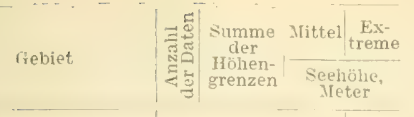

\section{Sorbus Aria Crantz.}

a) Cntere Arenze

Min

Nordwestkarpathen. | 3 | $857, \mathbf{2 9 0}$ | 280

\begin{tabular}{l|l|l|l|l|l|l|} 
Centralkarpathen. . & 7 & 2984 & 430 & 265
\end{tabular}

Nordostkarpathen . $|3| \quad 78|\mathbf{3 9 0}| 368$

Ostkarpathen ... . .

Südkarpathen .

\begin{tabular}{l|l|l} 
Südung. Gehirgsland & 3 & 1233
\end{tabular}

Bihargebirge.... $|1| 1 \mid 550$

Ung. Mittelgebirge . $|8| 1879$

Inselgebirge . . . . $\mid$

Ungarische Alpen . |

Kroatische Alpen . $|\approx 6| 10410$

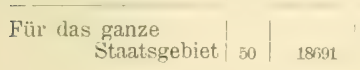

\section{b) Ohere fireuse}

Norilwestkarpathen | . |

Centralkarpathen. . $|24| 31327$

Nordostkarpathen . |

Ostkarrathen

Siidkarpathen . . . | .

Südung. Gebirgsland | $1 \mid 42: 8$

Bihargebirge.... . $|1| 1308$

Ung: Mittelgebirge $\cdot|*|$ * $\quad * \mid$ *

Inselgebirge .... $|*| *^{*} \quad * \mid *$

Ungarische Alpen . $|*|$ * * * * *

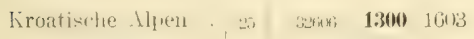

Fiir das ganze \begin{tabular}{l|l|l|l|l} 
Statsgebiet & 54 & 69469 & $\mathbf{1 2 9 0}$ & 1603
\end{tabular}

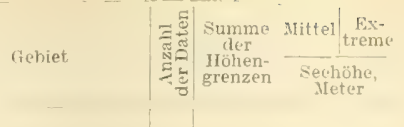

\section{Crataegus monogyna u. oxyacantlat}

Obere Grenze

Max.

Noriwestkarpathen.| $13 \mid 11399$ |' $880 \mid 1022$

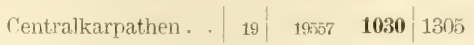

Nordostkarpathen $\mid$\begin{tabular}{l|ll|l}
5 & 4042 & $\mathbf{8 1 0}$ & 960
\end{tabular}

Ostkarpathen . . . $|21| 21864 \quad \mathbf{1 0 4 0} \mid 1329$

Südkaupathen . . . $|12| 113 \%$ 950 $\mid 1178$

\begin{tabular}{|l|l|l|l|l|l|l|l|l|} 
Siidung. Gebirgsland & 3 & 3316 & $\mathbf{1 1 1 0}$ & 1390
\end{tabular}

Bihargebirge. . . $|25| \quad 27048 \quad 1080 \mid 1219$

Ung. Mittelgebirge . $|*| * * \quad * \mid *$

Inselgebirge .... * * $\mid$ * * $\mid$ *

Ungarische Alpen . $|*|$ * * * $\mid *$

Kroatische Alpen . $18|15893,880| 1000$

Fiir das ganze

Staatsgebiet $|116| 114487 \quad 990 \mid 1390$

Prunus arium 1.

Obere Grenze.

Max.

Nordwestkarpathen | 1 | 891 || $\quad 891$

\begin{tabular}{l|l|l|l|l} 
Centralkarpathen. & 4 & 3470 & $\mathbf{8 7 0}$ & 951
\end{tabular}

\begin{tabular}{l|l|l|l|l|l} 
Norilostkarpathen & 3 & 2538 & 850 & 977
\end{tabular}

\begin{tabular}{l|l|l|l|l} 
Ostkarpathen . . . & 6 & 5695 & $\mathbf{9 5 0}$ & 1032
\end{tabular}

Siidkarpathen ... $3|3320| \mathbf{1 1 1 0} \mid 1304$

\begin{tabular}{ll|l|l|l|l|} 
Sitdung. Gebirgsland & fo & g065 & $\mathbf{1 0 1 0}$ & 1217
\end{tabular}

\begin{tabular}{|l|l|l|l|l|l} 
Bihargebirge. . . . & 7 & 5854 & $\mathbf{8 4 0}$ & 1169
\end{tabular}

Ung. Mittelgehirge . $\mid$\begin{tabular}{l|l|l|l} 
& 1 & 9 &
\end{tabular}

\begin{tabular}{l|l|l|l|l|} 
Inselgehirge . . . & 1 & 682
\end{tabular}

\begin{tabular}{l|l|l|l|l} 
Ungarische Alpen . & 1 & 862 & & \\
& & & &
\end{tabular}

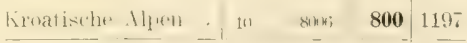

Fitr das ganze $\mid$ 


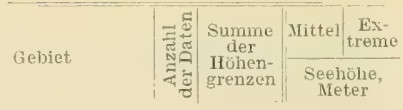

\section{Prumus Mahaleb I.}

obere direnze

Nordwestkarpathen. $\mid$\begin{tabular}{lll|l|l} 
& 1 & 439
\end{tabular}

Centrilkarpathen. . . .

Norilostkarpathen

Ostkalpathen

Süilkatpathen

Sürlung. Gebirgsland $|3| \quad 2649 \quad 880 \mid 1100$

Bihargebirge. . . . . . . . .

\begin{tabular}{|l|l|l|l|l} 
U11g. Mittelgebirge . & 8 & 5103 & $\mathbf{6 4 0}$ & 784
\end{tabular}

Inselgebirge

Ungarische Alpen

Kroatische Alpen : $|15| 10906$ i $730 \mid 1009$

Füir das ganze

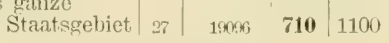

\begin{tabular}{|c|c|c|c|c|}
\hline \multicolumn{4}{|c|}{ Obere firenze } & \multirow{2}{*}{$\begin{array}{l}\operatorname{Max} \\
864\end{array}$} \\
\hline Norilvestkarpathen. & 6 & 4550 & 760 & \\
\hline Centralkarpathen. . & 8 & 6715 & 810 & 1061 \\
\hline Nordostkarpathen . & 1 & 808 & . & 808 \\
\hline Ostkarpathen ... & 9 & 8395 & 930 & 1055 \\
\hline Sürlkarpathen . . . & - & . & . & . \\
\hline Südung. Gehirgslank & . & . & . & . \\
\hline Bihargebirge . . . . & . & . & - & . \\
\hline Ung. Mittelgebirge . & - & . & . & . \\
\hline Inselgebirge . . . . & . & . & - & . \\
\hline Ungarische Alpen . & 1 & 690 & . & 690 \\
\hline Kroatische Alpen . & - & - & - & - \\
\hline $\begin{array}{l}\text { Für das ganze } \\
\text { Statsgebiet }\end{array}$ & 25 & 21158 & $8 \overline{50}$ & 1061 \\
\hline
\end{tabular}

\section{P'unus padus $\mathbf{L}$.}

Gebiet

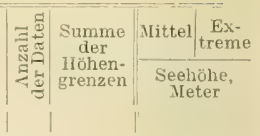

Prumus spinosa 1 .

Where Girenze

Max.

\begin{tabular}{l|l|lll} 
Noriwestkarpathen. & 1 & 604 & - & 664
\end{tabular}

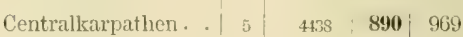

\begin{tabular}{l|l|ll|l} 
Nordostkarpathen . & 1 & $819 \quad$ & 819
\end{tabular}

\begin{tabular}{ll|l|l|l|l|} 
Ostkarpathen $\ldots$ &. & 5 & 5227 & $\mathbf{1 0 5 0}$ & 1118
\end{tabular}

Sïulkarpathen

Südung. Gebirgslanı .

\begin{tabular}{l|c|cc|c} 
Bihargebirge . . . & 11 & 11882 & $\mathbf{1 0 8 0}$ & 1316 \\
Ung. Mittelgelirge . & $*$ & $*$ & $*$ & $*$ \\
Inselgebirge . . . & $*$ & $*$ & $*$ & $*$ \\
Ungarische Alpen . & $*$ & $*$ & $*$ & $*$ \\
Kroatische Alpen . & 3 & 2343 & $\mathbf{7 8 0}$ & 838 \\
Für das ganze & & & &
\end{tabular}

Berheris vulgaris $\mathbf{L}$.

\section{Oheve Grenze}

Max.

\begin{tabular}{l|l|l|l|l} 
Nordwestkarpathen. & 1 & 500 & $\vdots$ & 526
\end{tabular}

\begin{tabular}{ll|l|l|l|l} 
Centralkarpathen . . & 1 & 768 &. & 768
\end{tabular}

Nordostkarpathen . . .

\begin{tabular}{l|l|l|l|l|l} 
Ostkarpathen... & 4 & 3526 & 880 & 1003
\end{tabular}

Sïdkarpathen

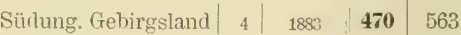

\begin{tabular}{l|l|l|l|l|l|} 
Bilıargebirge . . . & 1 & 432 & - & 432
\end{tabular}

\begin{tabular}{l|l|l|l|l} 
Ung. Mittelgebirge . & 1 & 823 & $\cdot$ & $\cdot 823$
\end{tabular}

Inselgebirge . . . . . .

Ungarische Alpen . .

\begin{tabular}{l|l|l|l|l|l|} 
Kroatische Alpen . & 1 & 878 & & 878
\end{tabular}

Für das ganze \begin{tabular}{l|l|l} 
Statsgebiet & 13 & 8836
\end{tabular} 


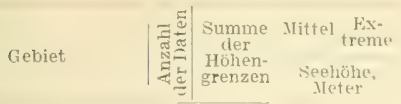

\section{Eronymus europaens $\mathbf{L}$.}

\section{Obere Grenze}

Centralkarpathen.

\begin{tabular}{l|l|l|l|l|} 
Centralkarpathen. . & 1 & 60, & $\cdot$ & 630
\end{tabular}

Nordostkarpathen . : * : (4t8)

\begin{tabular}{ll|l|l|l|} 
Ostkarpathen ... & 4 & 3160 & $\mathbf{7 9 0}$ & 852
\end{tabular}

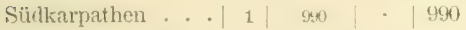

Stizdung. Gehirgsland $\mid$\begin{tabular}{l|l|l|l}
$\mid$ & si1 & $\cdot$ & 861
\end{tabular}

\begin{tabular}{l|l|l|l|} 
Bihargehirge . . . & 1 & 986 & 986
\end{tabular}

Ung. Mittelgebirge . $1 \mid$ s71 $|\cdot| 871$

Inselgebirge ..... . . . $\mid$ |(261)

Ungarische Alpen . . . . . . . .

\begin{tabular}{l|l|l|l|l} 
Kroatische Alpen & 1 & 788 & $*$ & 788 \\
\hline Fiir las ganze & & &
\end{tabular}

\section{Eтопущи rerrucosus sсор.}

Obere firenz:

Nordwestkarpathen. $\cdot$. .

Centralkarpathen. . 1 | $400 . \mid 400$

Norlostkarpathen $\cdot|\cdot|$

Ostkarnathen ... $8 \mid$ 75fī 950 1049

Süilkarplathen . . . 1 | 1196 . 1196

Südung. Gebirgsland $|1| 563 .+563$

Bihargebirge . . | $4 \mid 3612$ | 900| 1020

Ung: Mittelgebirge . $|1| 652, \cdot \mid 652$

Inselgehirge . . . $1|380| \quad \mid 380$

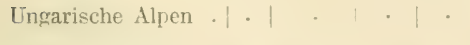

Kroatische Alpen . $|1| 638 \quad \cdot \mid 638$

Für das ganze \begin{tabular}{l|l|l|l|l} 
Staatsgebiet & 18 & 15006 & 830 & 1196
\end{tabular}

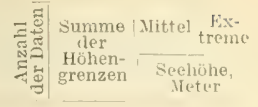

\section{Staphylea pinuata I.}

\begin{tabular}{|c|c|c|c|c|}
\hline \multicolumn{4}{|c|}{ Obere drenze } & \multirow{2}{*}{$\begin{array}{l}\text { Max. } \\
736\end{array}$} \\
\hline Norlwestkarpathen. & 1 & 736 & • & \\
\hline Centralkarpathen. . & 1 & 510 & $\cdot$ & 510 \\
\hline Norilostkarpathen . & 1 & 566 & $\cdot$ & 566 \\
\hline Ostkaryathen . . & 1 & 689 & $\cdot$ & 682 \\
\hline Sïitlkarpathen . . . & . & . & . & \\
\hline Süclung. Gebirgsland & 1 & 672 & $\cdot$ & 672 \\
\hline Bihargebirge. . . & 1 & 520 & $\cdot$ & 520 \\
\hline Ung. Mittelgebirge . & 1 & 608 & $\cdot$ & 608 \\
\hline Inselgebirge . . . . & 1 & 682 & $\cdot$ & 682 \\
\hline Ungarische Alpen . & . & . & $\cdot$ & \\
\hline Froatische Alpen . & 4 & 2967 & 570 & 614 \\
\hline Erdélyer Becken . . & 1 & 685 & $\cdot$ & 685 \\
\hline $\begin{array}{l}\text { das ganze } \\
\text { Staatsgebiet }\end{array}$ & 13 & 7928 & 610 & \\
\hline
\end{tabular}

Rhamnus frangula $L_{\text {. }}$.

obere Girenze Nax.

\begin{tabular}{l|l|l|l|l} 
Norilwestkarpathen & 1 & 679 & 079
\end{tabular}

Centralkarpathen. - $|1| 1030, \cdot 1030$

Nordostkarpathen

Ostkarpathen $\cdots|7| \quad 6991: 900 \mid 1000$

Südkarpathen . . | 2 2 1841 920 | 1005

Südung. Gebirgsland| .

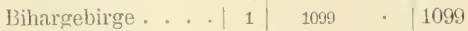

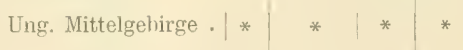

Inselgehirge .

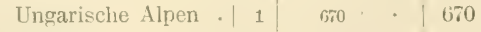

| Irroatische Nipen .| 1 (10 | * 940

\begin{tabular}{l|l|l|l|l} 
Für llas ganze & & & & \\
Staatsgebiet & 14 & 12550 & $\mathbf{9 0 0}$ & 1099
\end{tabular} 
Gebiet.

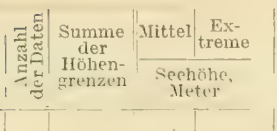

\section{Cornus mas $\mathbf{L}$.}

\begin{tabular}{|c|c|c|c|c|}
\hline \multicolumn{4}{|c|}{ Obere firenze } & Max. \\
\hline Nordwestkarpathen. & 1 & 512 & . & 512 \\
\hline Centralkarpathen. & 6 & 4561 & 760 & 1000 \\
\hline Norlostkarpathen . & 1 & 625 & - & 625 \\
\hline Ostkarpathen . . & . & . & - & - \\
\hline Südkarpathen . . & 1 & 1102 & - & 1102 \\
\hline Südung. Gebirgsland & 6 & 4719 & 790 & 981 \\
\hline Bihargebirge . . . & 3 & 2182 & 730 & 986 \\
\hline Ung. Mittelgebirge & 6 & 4760 & 790 & 895 \\
\hline Inselgebirge . & 1 & 953 & . & 953 \\
\hline Ungarische Alpen . & - & . & . & - \\
\hline Kroatische Alpen & 18 & 13644 & 760 & 1010 \\
\hline $\begin{array}{l}\text { Für das ganze } \\
\text { Stantsgebiet }\end{array}$ & 43 & 33058 & 770 & 1102 \\
\hline
\end{tabular}

Cornus sauguinea $\mathrm{L}$.

bbere Grenze

\begin{tabular}{|c|c|c|c|c|}
\hline & \\
\hline ordwestkarpathen. & . & . & & \\
\hline ntralkarpathen. . & 4 & 2761 & 690 & 820 \\
\hline prdostkarpathen & 1 & 823 & . & \\
\hline tkarpathen ... & 6 & 5903 & 980 & 11 \\
\hline idkarpathen ... & 1 & 1180 & . & 118 \\
\hline iclung. ('ebirgslanil & 1 & 1037 & . & (1) \\
\hline ihargebirge . . & 9 & 6986 & 780 & \\
\hline ng. Mittelgebirge & 1 & 940 & & \\
\hline aselgebirge . . & 1 & 484 & . & \\
\hline Ungarische Alpen & . & . & . & \\
\hline roatische $\mathrm{Al}_{\mathrm{j}} \mathrm{en}$ & 12 & shist & 670 & \\
\hline $\begin{array}{l}\text { das ganze } \\
\text { Staatsgebiet }\end{array}$ & 36 & 28170 & 780 & \\
\hline
\end{tabular}

\section{Ribes alpinum $\mathbf{I}$.}

\section{a) Untere Grenze}

Min.

\begin{tabular}{l|l|l} 
Nordwestkarpathen. & 1 & 1047
\end{tabular}

\begin{tabular}{|l|l|l|l|l} 
Centralkarpathen. . & 7 & 5264 & $\mathbf{7 5 0}$ & 450
\end{tabular}

Nordostkarpathen . .

\begin{tabular}{|l|l|l||l|l|}
\hline Ostkarpathen $\cdots$ & 3 & 3357 & 1120 & 855
\end{tabular}

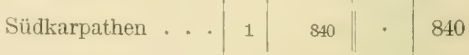

Südung. Gebirgsland ! .

\begin{tabular}{|l|l|l||l|l} 
Bihargehirge . . . & 1 & 618 &. & 618
\end{tabular}

Ung. Mittelgebirge .

Inselgebirge

Ungarische Alpen . .

Kroatische Alpen

1

571

571

Für das ganze

Staat.sgebiet. $14 \mid \begin{array}{llll}11698 & \mathbf{8 1 0} & 450\end{array}$

b) Ghere drenge

$\operatorname{Max}$

\begin{tabular}{l|l|l|l|l|l|} 
Nordwestkarpathen. & 1 & 1414 & $\cdot$ & 1414
\end{tabular}

\begin{tabular}{ll|l|l|l|l} 
Centralkarpathen. . & 5 & 8005 & $\mathbf{1 6 0 0}$ & 1780
\end{tabular}

Nordostkarpathen

Ostkarpathen

Südkarpathen . . . 1

$1769 \quad \cdot \mid 769$

Siidung. Gebirgsland

Bihargebirge .

Ung. Mittelgebirge .

Inselgebirge

Ungarische Alpen

liroatirche Alpen

$114+4$

$1+16$

Für das ganze

\begin{tabular}{l|l|l} 
Staatsgebiet & 8 & 12634
\end{tabular}

$1580 \quad 1780$ 


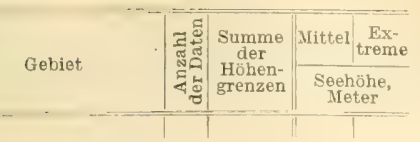

\section{Syringa vnlgaris $\mathbf{L}$.}

a) Untere Grenze

Min.

Nordwestkarpathen. $\left.\right|_{-1} \quad-\quad|-|-$

Centralkarpathen

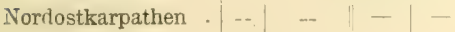

Ostkarpathen ... -

\begin{tabular}{|l|l|l|l|l} 
Südkarpathen . . . & 3 & 2200 & $\mathbf{7 3 0}$ & $\mathbf{7 2 2}$
\end{tabular}

\begin{tabular}{|l|l|l|l|l} 
Südung, Gebirgsland & 1 & 51 & . & 51
\end{tabular}

\begin{tabular}{|l|l|l|l|l|l|l|l|} 
Bihargebirge . . . & 1 & 320 & 320
\end{tabular}

Ung. Mittelgebirge . $-1+1-1-$

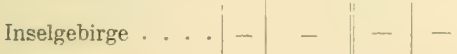

Ungarische Alpen . $-1-1 \quad-\quad-$

Kroatische Alpen : - $-1 \mid-$

Für das ganze

b) Obere (irenze

Nordwestkarpathen. $1-1+$ Max.

Centralkarpathen. . 1 - - $1-1-$

Nordostkarpathen $\cdot|-| \quad \ldots \quad \ldots$

Ostkarpathen ... - - $-1-\mid-$

\begin{tabular}{|l|l|l|l|l} 
Südkarpathen ... & 1 & 1190 & $\cdot 1190$
\end{tabular}

\begin{tabular}{l|l|l|l|l} 
Südung. Gebirgsland & 9 & 9215 & $\mathbf{1 0 2 0}$ & 1305
\end{tabular}

Bihargehirge.... $1 \mid 1020 / \cdot 1020$

Ung. Mittelgebirge . $-1-1-1-$

Inselgebirge . . . - - - $1-1-$

Ungarische Alpen . $|-|-|-|-$

Kroatische Alpen . $-1-1-1-$

Für das ganze \begin{tabular}{|l|l|l|l|l|l} 
Staatsgebiet & 11 & 11426 & 1040 & 1305
\end{tabular}

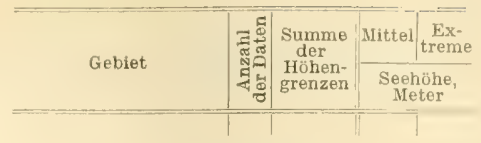

Syringa Josikaea Jacq. fll.

a) Entere Grenze

Min.

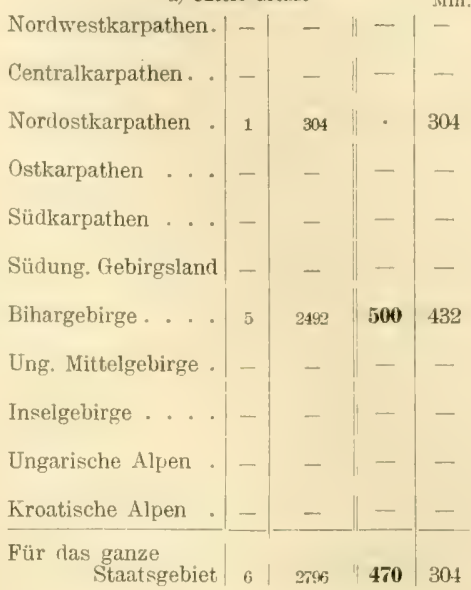

b) obere Arenge

- Max.

Nordwestkarpathen. $|-|-|-|-$

Centralkarpathen. . $|-| \quad \ldots \quad||-\mid-$

\begin{tabular}{l|l|l|l|l|l|l|} 
Norilostkarpathen . & 1 & 568 || & 568
\end{tabular}

Ostkarpathen ... $-|-||-|-$

Sïdkarpathen ... . -

Südung. Gebirgsland $|-|-||-\mid-$

\begin{tabular}{|l|l|l|l|l} 
Bihargebirge . . . & 4 & 3319 & $\mathbf{8 3 0}$ & 955
\end{tabular}

Ung. Mittelgebirge . $|-|-|-|-$

Inselgebirge .... - - - $\mid-1-$

Ungarische Alpen . - - -

\begin{tabular}{l|l|l|l|l} 
Kroatische Alpen : & - & - & - & - \\
\hline Für das ganze & & & & -
\end{tabular} 


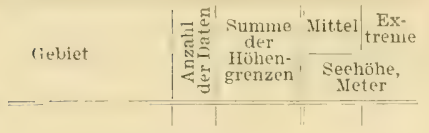

Ligustrnm vulgare $\mathbf{L}$.

Obere Grenze

\begin{tabular}{|c|c|c|c|c|}
\hline & \\
\hline Nordwestkarpathen. & 1 & 753 & . & 753 \\
\hline Centralkarpathen. & 1 & 784 & - & \\
\hline Nordostkarpathen . & 1 & 808 & $\cdot$ & \\
\hline Ostkarpathen ... & 1 & 921 & - & \\
\hline Südkarpathen . . . & . & . & . & \\
\hline Südung. Gebirgsland & . & . & . & \\
\hline Bihargebirge. . . & 1 & 479 & . & \\
\hline Ung. Mittelgebirge . & 1 & 740 & . & \\
\hline Inselgebirge . . & 1 & 484 & - & \\
\hline Ungarische Alpen . & . & . & . & \\
\hline H roatische Alpen & 3 & 1838 & 610 & \\
\hline $\begin{array}{l}\text { Für das ganze } \\
\text { Staatsgebiet }\end{array}$ & 10 & 6807 & 680 & \\
\hline
\end{tabular}

\section{Sambueus uigra $\mathbf{L}$.}

Obere Grenze

\begin{tabular}{|c|c|c|c|c|}
\hline Nordwestkarpathen. & 1 & 881 & . & 881 \\
\hline Centralkarpathen. . & 14 & 11982 & 860 & 1040 \\
\hline Nordostkarpathen . & 6 & 6018 & 1000 & 1088 \\
\hline Ostkarpathen . . & 16 & $183 \%$ & 1150 & $\mid 1369$ \\
\hline Südkarpathen . . . & 17 & 19236 & 1130 & 1494 \\
\hline Südung. Gebirgsland & 12 & 12368 & 1020 & 1282 \\
\hline Bihargebirge. . & 19 & 17979 & 950 & 1306 \\
\hline Ung. Mittelgebirge . & 1 & 1005 & . & 1005 \\
\hline Inselgebirge . . & 1 & 682 & . & 682 \\
\hline Ungarische Alpen & * & * & * & 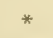 \\
\hline Kroatische Alpen & 13 & $11: m$ & 920 & $1: 01$ \\
\hline $\begin{array}{c}\text { Für das ganze } \\
\text { Staatsgebiet }\end{array}$ & 100 & +18 & 1000 & \\
\hline
\end{tabular}

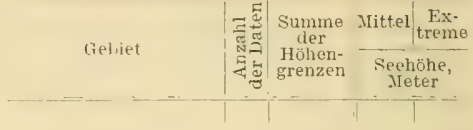

Sambueus racemosa $\mathbf{L}$.

a) Untere Grenze

Min.

Nordwestkarpathen. $\mid$\begin{tabular}{l|l|l|} 
& 1 & 530
\end{tabular}

\begin{tabular}{l|l|l|l|l|l} 
Centralkarpathen. & 6 & 3017 & $\mathbf{5 0 0}$ & 292
\end{tabular}

\begin{tabular}{l|l|l|l|l} 
Nordostkarpathen - 10 & 5232 & $\mathbf{5 2 0}$ & 236
\end{tabular}

\begin{tabular}{|l|l|l|l|l|l|l|} 
Ostkarpathen ... & 1 & 630 &. & 630
\end{tabular}

\begin{tabular}{|l|l|l|l|l} 
Sükkarpathen . . . & 6 & 5069 & $\mathbf{8 5 0}$ & 528
\end{tabular}

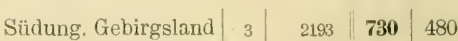

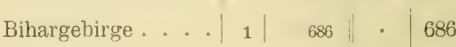

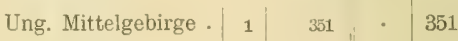

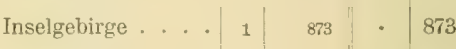

Ungarische Alpen

\begin{tabular}{llll|l} 
Kroatische Alpen . 2 1105 & $\mathbf{5 5 0}$ & 462
\end{tabular}

Für das ganze

b) Obere Grence

Nax.

\begin{tabular}{l|l|l|l|l|l} 
Nordwestkarpathen. & 2 & 2345 & $\mathbf{1 1 7 0}$ & 1243
\end{tabular}

\begin{tabular}{|l|l|l|l|l}
\hline Centralkarpathen. . & 17 & 22015 & $\mathbf{1 3 0 0}$ & 1506
\end{tabular}

Nordostkarpathen , $19|25409 \quad| \mathbf{1 3 4 0} 1570$

\begin{tabular}{|l|l|l|l|l} 
Ostkarpathen $\ldots .$. & 97 & 39501 & $\mathbf{1 4 6 0}$ & 1774
\end{tabular}

Südkarpathen ... $30|\quad 46362||\mathbf{1 5 5 0}| 1720$

\begin{tabular}{ll|l|l|l} 
Südung, Gebirgsland & 10 & 14177 & $\mathbf{1 4 2 0}$ & 1599
\end{tabular}

\begin{tabular}{ll|l|l|l|l} 
Bihargebirge . . . & 19 & 26408 & $\mathbf{1 3 9 0}$ & 1545
\end{tabular}

Ung. Mittelgebirge . $*$ * $|*||*| *$

Inselgebirge .... * $^{*} \quad * \quad \|^{*} \mid *$

Ungarische Alpen

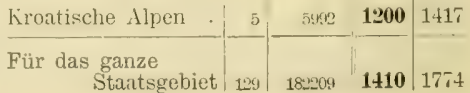




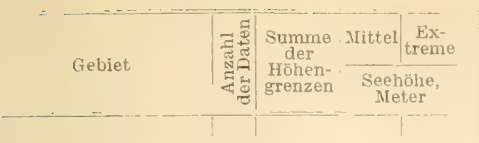

Viburnum Lantana $\mathbf{L}$.

\section{obere frenze}

Nordwestkarpathen. $|1| \quad 768$

Centralkarpathen . . $10 \mid 8164$

Nordostkarpathen . . . .

\begin{tabular}{l|l|l} 
Ostkarpathen ... & 1 & 1
\end{tabular}

Südkarpathen ... $|1| 1170$

Südung. Gebirgsland $\left|{ }_{4}\right|$. 3892

Bihargebirge... $|7| 7.646$

Ung. Nittelgebirge · $|1| 932$

Inselgebirge .... $|*| *$

Ungarische Alpen . $\cdot$.

Kroatische Alpen : $|5| \quad 4698$

Für das ganze Staatsgebiet $30 \mid 26931$

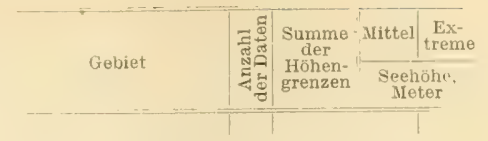

Viburnum opulus $\mathbf{L}$.

obere Grenze

\begin{tabular}{l|l|ll|l} 
Nordwestkarpathen & 4 & 3088 & $\mathbf{7 7 0}$ & 887
\end{tabular}

\begin{tabular}{l|l|l|l|l|l|} 
Centralkarpathen. & 8 & $75+2$ & 940 & 1158 \\
\hline
\end{tabular}

\begin{tabular}{|l|l|ll|l} 
Nordostkarpathen . & 3 & 2456 & $\mathbf{8 2 0}$ & 829 \\
\hline
\end{tabular}

. $891 \mid$ Ostkarpathen ... . $13 \mid$\begin{tabular}{lll|l}
13074 & 1010 & 1130
\end{tabular}

. $|1170|$ Südkarpathen ...| $1 \mid$ 6ro . $\mid 670$

970 $|1200|$ Südung. Gebirgsland $|1| 450|\cdot| 450$

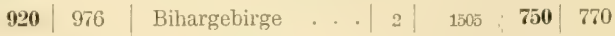

. 932 Ung. Mittelgebirge . $|1| 751$ | $\mid 751$

* | * | Inselgebirge ... . $1 \mid$ 1 $44 ! \cdot 444$

- . $\mid$ Ungarische Alpen . $|1|$ 526 . $\mid 526$

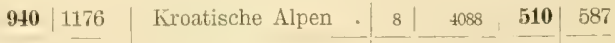

Für das ganze

Staatsgebiet| 43 | $34594 \quad|| \mathbf{8 0 0} 1158$ 



\section{Register zum zweiten Band.}

Ibies alba Mill. 2, 10, 25, 38, 50, 60, 72. $88,92,97,123$.

- pectinata $D C .=$ A. alba Mill.

Acer campestre L. 7, 22, 34, 47, 68, 80, 86, 111, 138.

- monspessulanum L. 68, 112.

- obtusatum W. et K. 111.

- platanoides L. $6,21,34,46,5 \overline{7}, 68$, $79,110,137$.

- Pseudoplatanus L. 7, 21, 34, 46, 57, $68,80,110,138$

- tataricum L. 36. 139.

Alnus glutinosa Gärtn. 5, 19, 31, 44, 55. $66,78,86,107,13$.

- incana Willd. 6, 19, 31, 44. 56. 66. 78, $108,134$.

- viridis DC. $32,45,56,66,78,94,135$.

Amelanchier ovalis Med. 117.

Aronia $=$ Amelanchier.

Berberis vulgaris L. 142.

Betula pubescens Ehrh. 18.

- verrucosa Ehrh. 18, 31, 44, 55, 66 . 77, 107, 134.

Carpinus Betulus L. 5, 18, 30, 43, 54, 65, $76,85,89,106,133$.

- duinensis Scop. 65, 106, 133.

- orientalis Lam. $=\mathrm{C}$. duinensis Scop.

Castanea sativa Mill. 89, 105.

Celtis australis L. 115.

Cornus mas L. 117, 144.

- sanguinea L. 144.

Corylus Avellana L. 18, 31, 43, 55, 65. 77, $107,134$.

- Colurna L. 65.

Cotinus Cogyggria Scop: 69, 87, 115.

Crataegus monogyna Jacq. 8, 23, 48, 81, $114,141$.

- oxyacantha L. $8,23,48,81,114,141$.

Cytisus Laburnum L. 117.

Evonymus europaeus L. 143.
Evonymus verrucosus Scop. 143.

Fagus silvatica $L .4,15,28,41,53,62,73$, $83,88,91,93,100,120,129$.

Ficus Carica I. 115.

Fraxinus excelsior L. 7, 22, 35, 47, 57, 68, $80,86,112,139$.

Ornus L. 69, 87, 90, 112, 139.

Ilex aquifolium L. 116.

Juglans regia $\mathrm{L}$. $64,10 \overline{5}$.

Juniperus communis L. 14, 27, 40, 99, 128. nana IVilld. $3,14,28,40,52,100,128$. Oxycedrus L. 100.

- Sabina L. 53, 73, 100.

Laburnum vulgare Griseb. = Cytisus La. burnum $I_{\text {s. }}$

Larix decidua Mill. 13, 93, 127. europaea DC. $=$ L. decidua Mill.

Laurus nobilis L. 115 .

Ligustrum vulgare L, 146.

0lea europea L. 117.

Ostrya carpinifolia Scop. 106.

Paliurus aculeatus Lam. 116.

Phillyrea latifolia L. 117.

Picea excelsa Lk. 1, 9, 24, 37, 49, 59, 71 $92,95,122$.

Pinus Cembra L. 12, 26, 39, 51, 61, 126. montana Mill. 3, 12, 26, 39, 51, 61, 73, 99. 126.

nigra Arn. 60, 98, 125.

- silvestris L. 3, 11, 93, 98, 124.

Pirus communis L. 139.

- malus L. 140.

Pistacia Terebinthus L. 115.

Populus tremula L. 6, 20, 32, 45, 79, $108,135$.

Prunus avium L. 141.

Mahaleb L. 87, 114, 142.

- padus L. 142.

- spinosa L. 114, 142.

(quercus Cerris L. 17, 64, 76, 85, 89, 104, 132 
Quercus conferta Kit. 64, 76, 132.

- Ilex I. 104.

- lanuginosa Lam. 17, 76, 84, 89, 103, 132.

- penduculata Ehrh. = Qu. IRobur L.

- pubescens Willd. $=\mathrm{Qu}$. lanuginosa Lam.

Robur L. $5,16,30,42,54,63,75,81$, $89,103,131$

- sessiliflora Salisb. 4. 16, 29, 42, 54, $63,75,83,88,93,102,131$.

Rhamnus alpina L. 116.

- cathartica I. 116.

- frangula L. 143.

Rhus Cotinus L. = Cotinus Coggygria Scop.

Ribes alpinum L. 144.

Salix alba L. 78, 108, 135.

- Caprea L. 19.

- pentandra L. 45 .

- silesiaca Willd. 19.

Sambucus nigra L. 118, 146 .

Sambucus sacemosa L. 23, 36, 48, 58, 70 , $82,118,146$.
Sorbus Aria Crantz. 22, 113, 141.

- aucuparia L. 7, 22. 35, 47, 58, 69, $81,113,140$.

-. torminalis Crantz. $69,87,113,140$.

Staphylea pinnata L. 143.

Syringa Josikaea Jacq. fil. 35, 81, 145.

- vulgaris L. 70, 82, 145.

Taxus baccata L. 14, 27, 62, 99, 128.

Tilia argentea Desf. $=\mathrm{T}$. tomentosa Mönch.

- grandifolia Ehrh. = T. platyphylla Scop.

- Cordata Mill. 21, 33, 67, 109, 137.

- parvifolia Ehrh. = T. cordata Mill.

- platyphylla Scop. 137.

- tomentosa Mönch. 67, 137.

Ulmus campestris L. $=$ U. glabra Mill.

- glabra Mill. 136.

- montana With = U. scabra Mill.

- scabra Mill. 6, 20, 33, 46, 56, 67, 79 . 86, 109, 136.

Viburnum Lantana L. 147.

- opulus L. 118, 147.

Vitis vinifera L. 119. 

Fekete-Blattny. Die Verbreitung der forstlich wichtigen Bäume und Sträucher im ungarischen Staate.

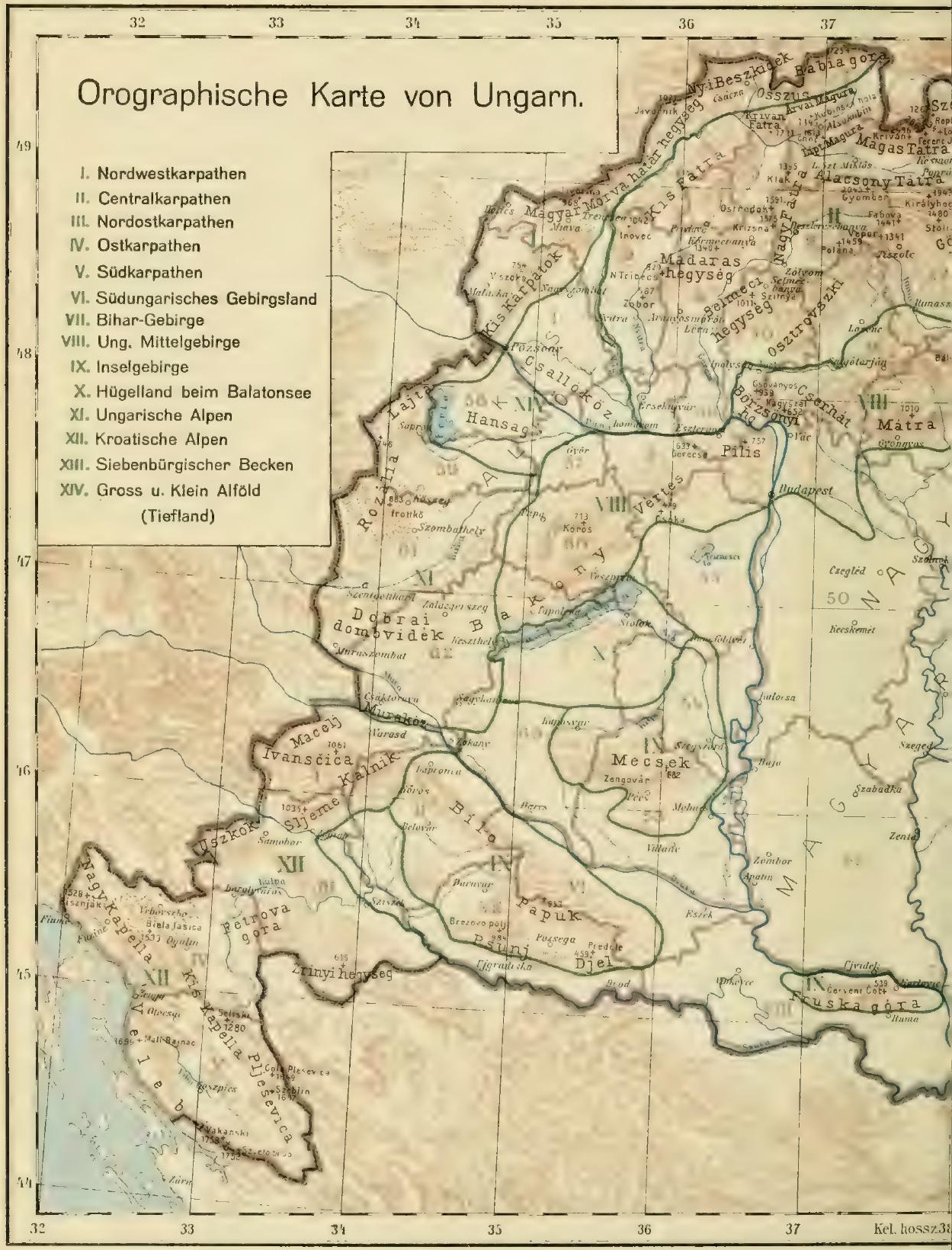




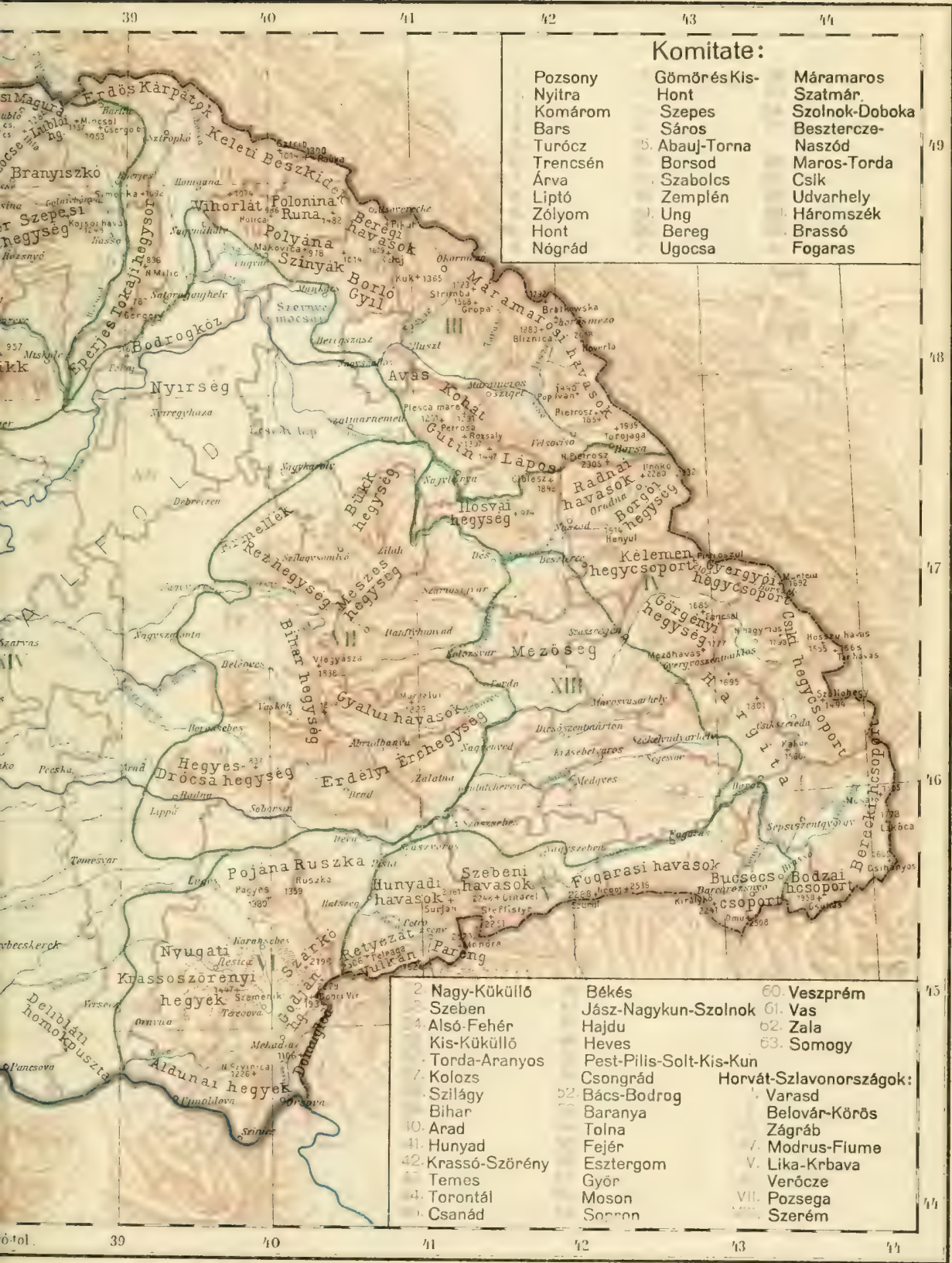




Fekete-Blattny: Die Verbreitung der forstlich wichtigen Bäume und Sträucher im ungarischen Staate.

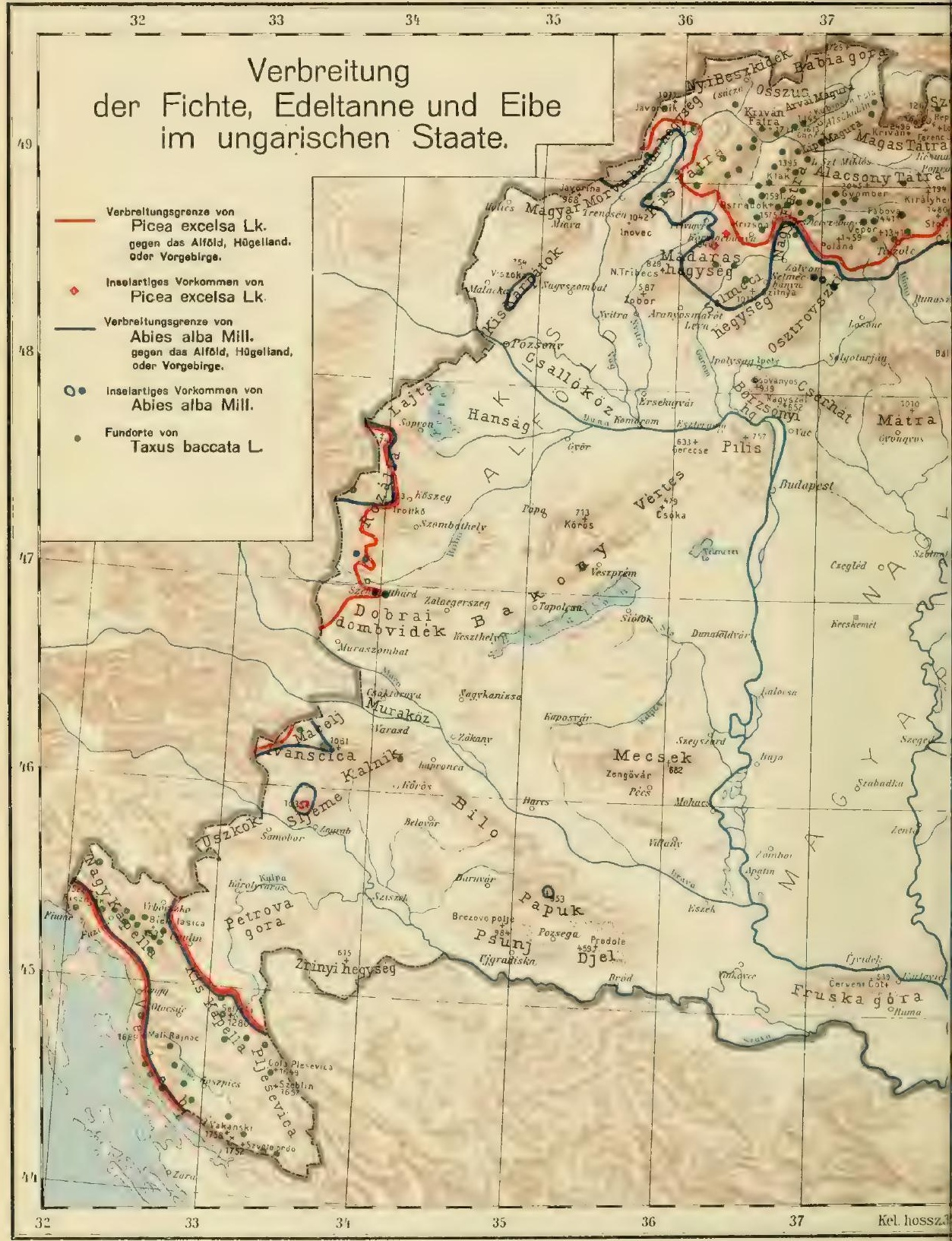




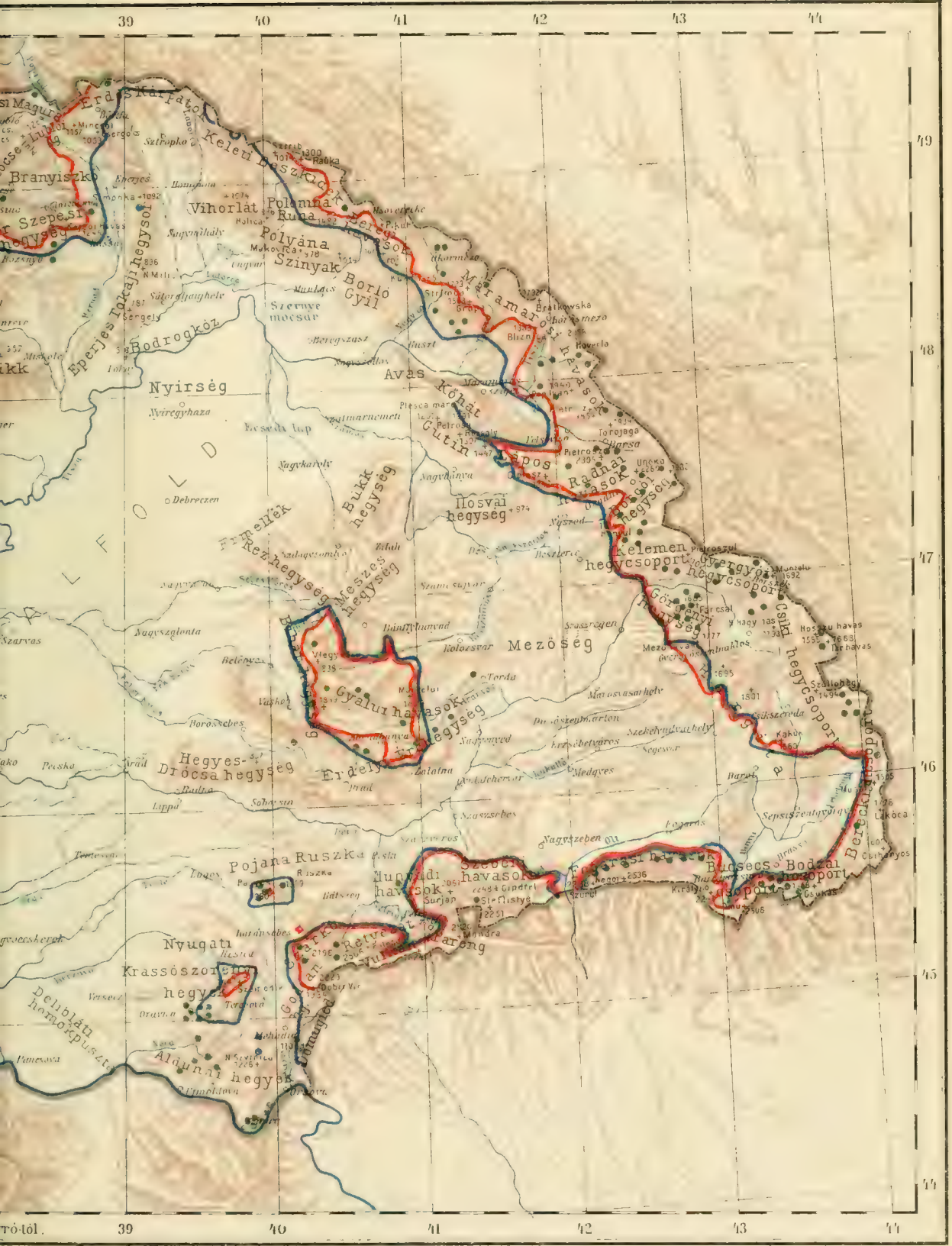



Fekete-Blattny: Die Verbreltung der forstlich wichtigen Bäume und Sträucher im ungarischen Staate.

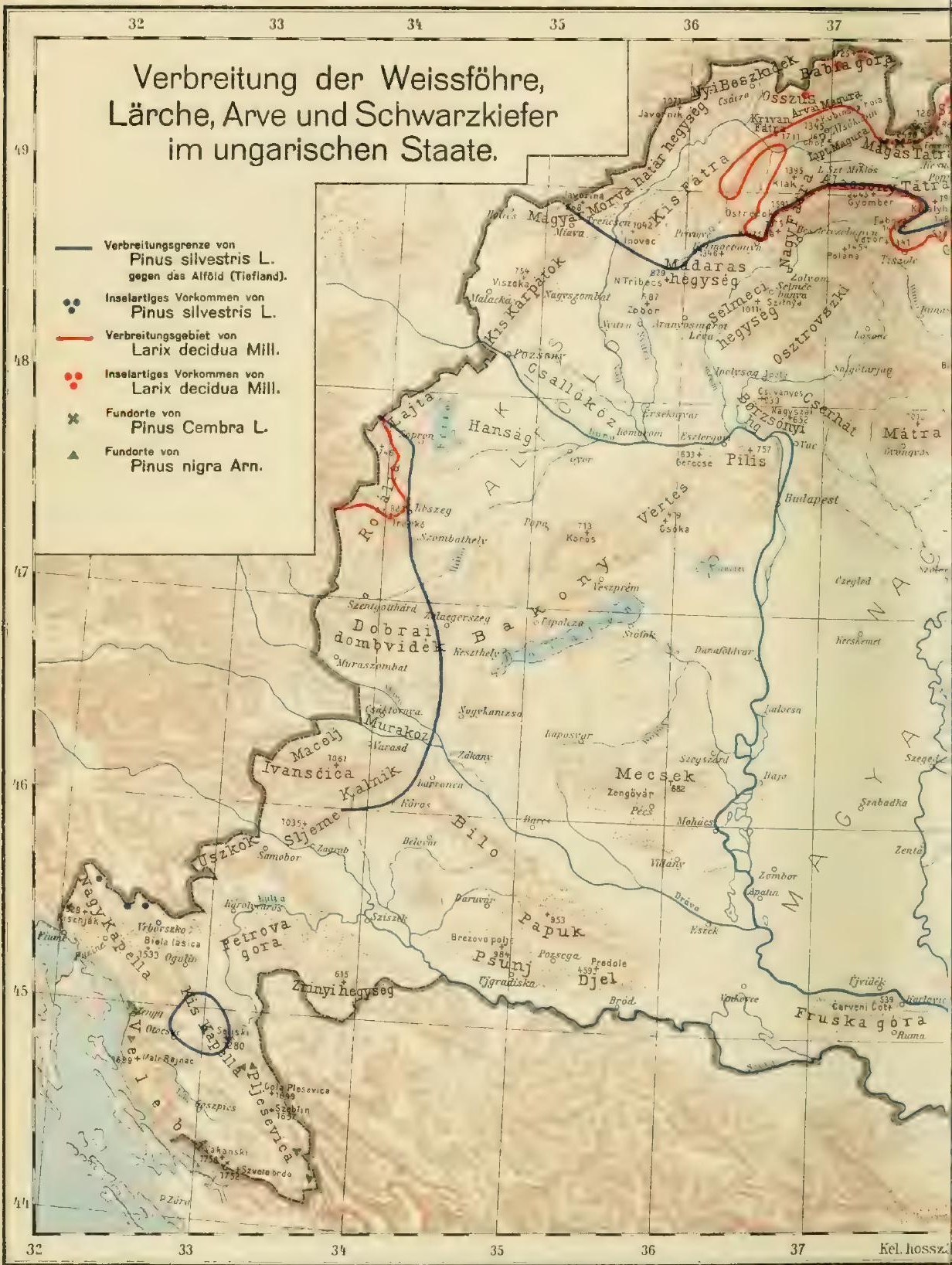


Karte III.

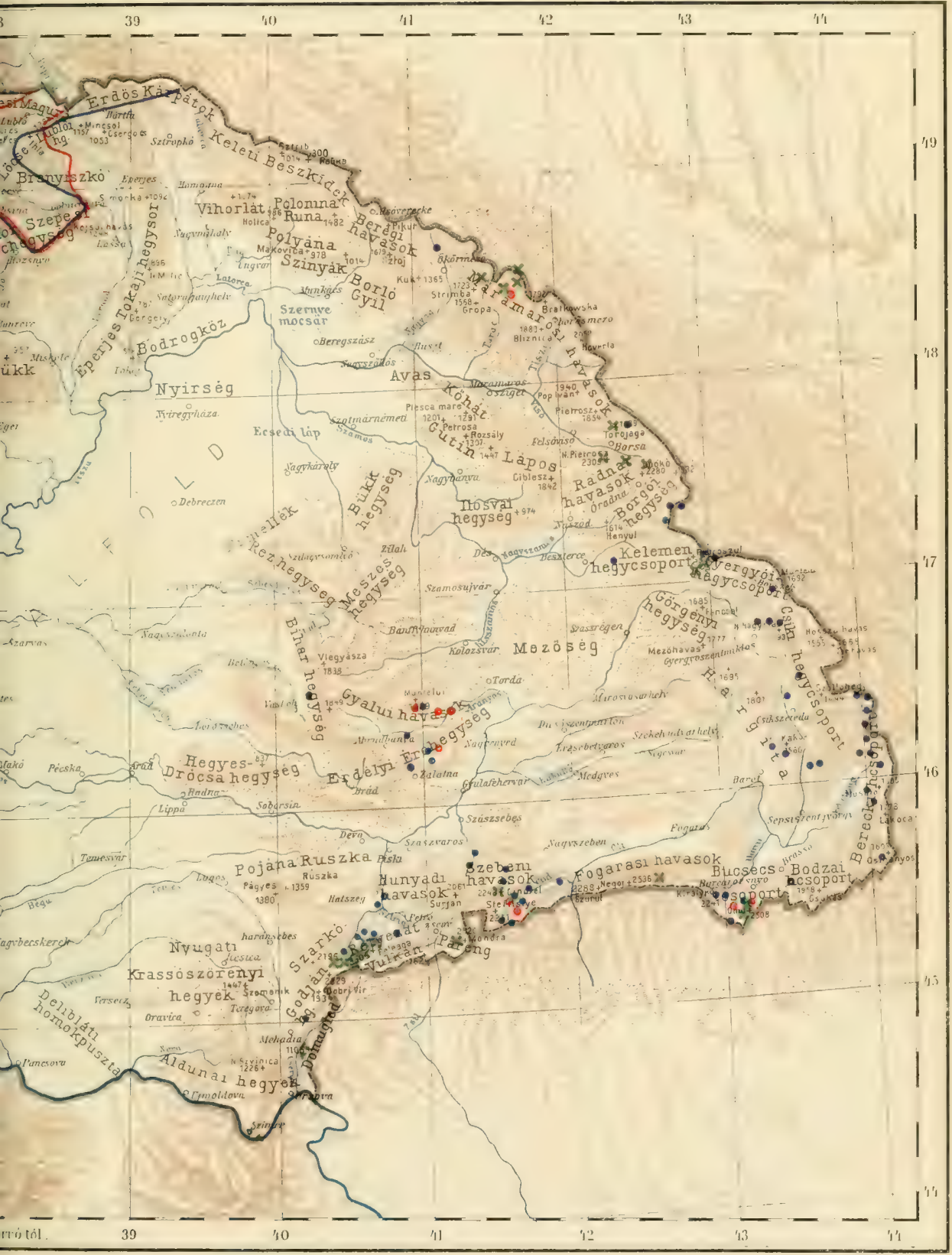




\section{,}




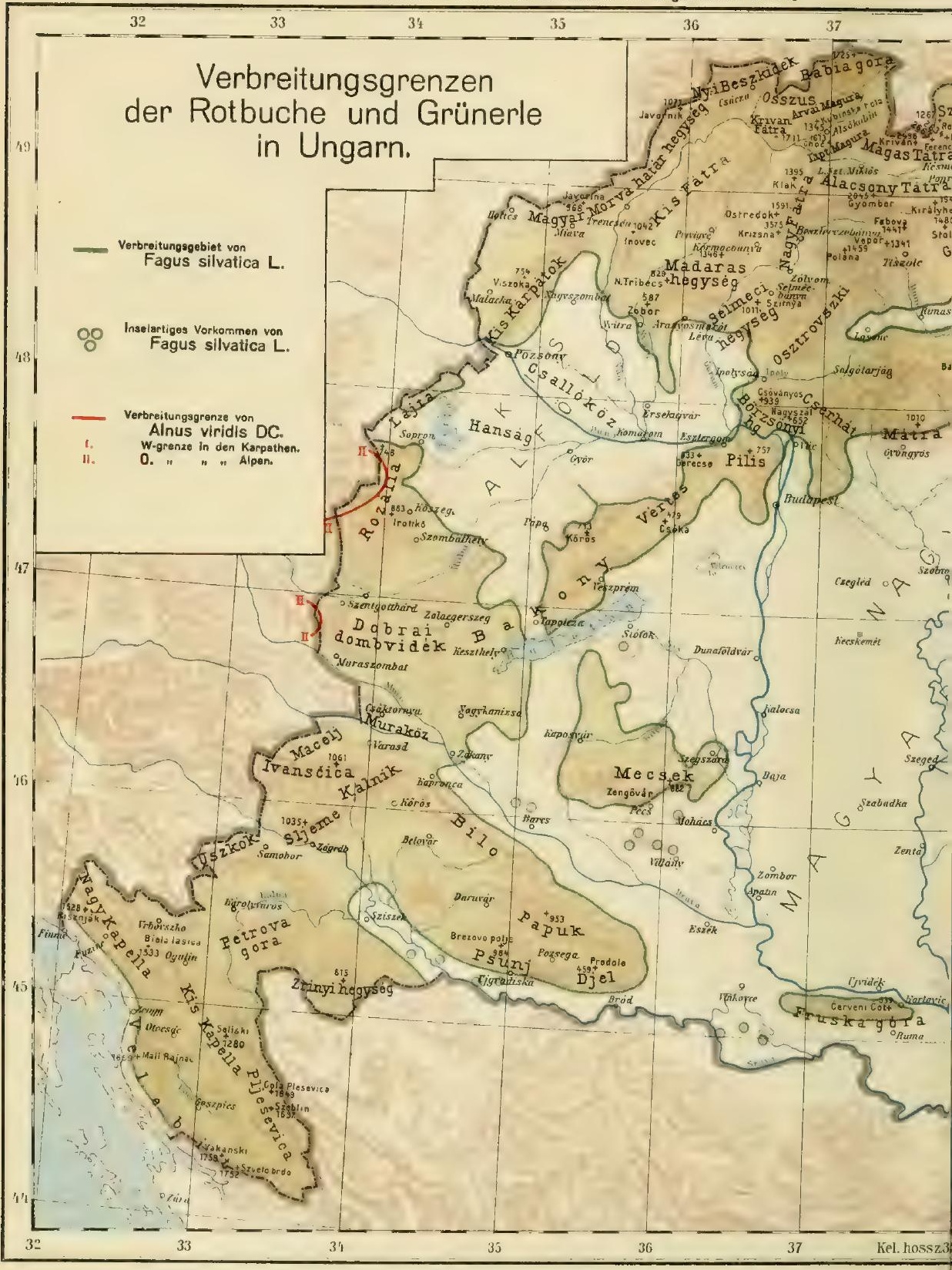




Fekete-Blattny: Die Verbreitung der forstlich wichtigen Bäume und Sträucher im ungarischen Staate.

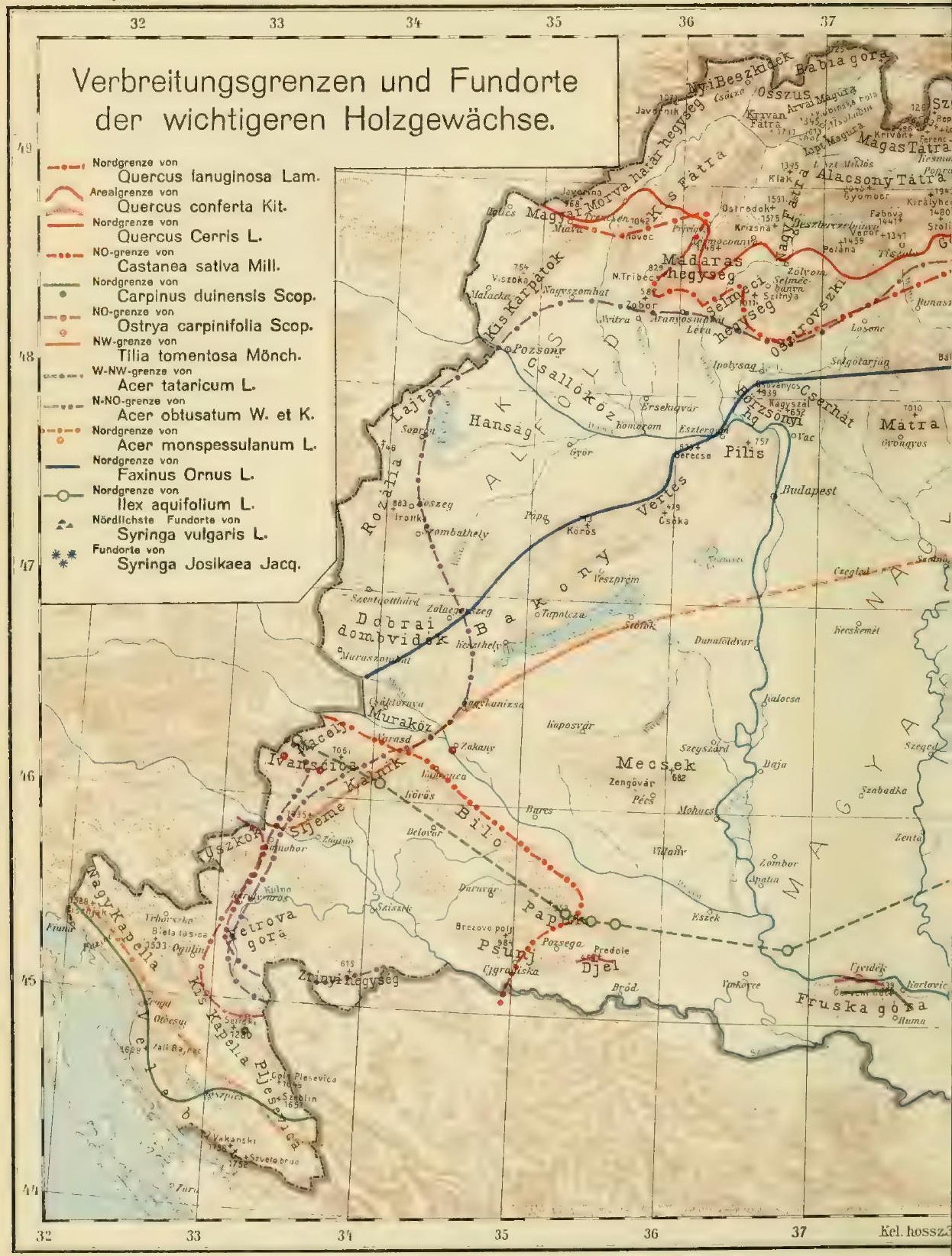




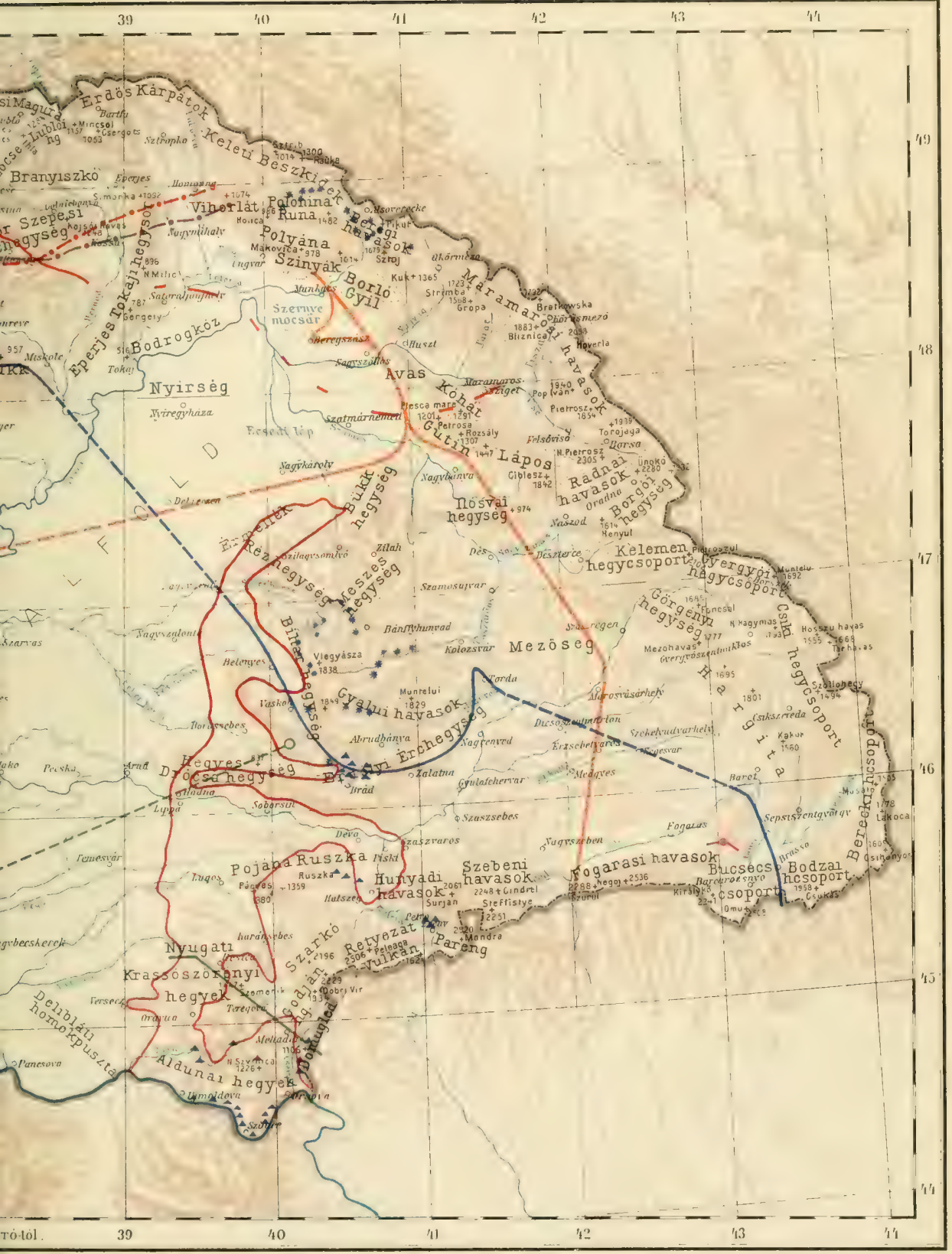






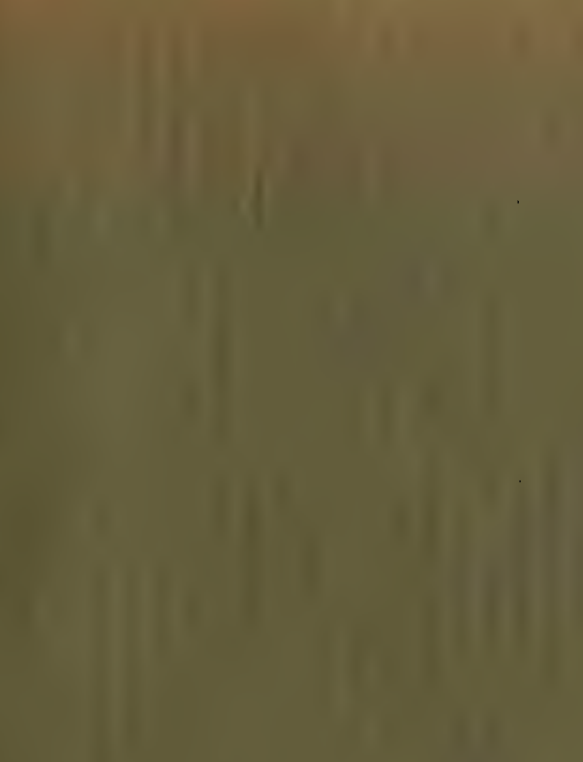

a) 1.1/

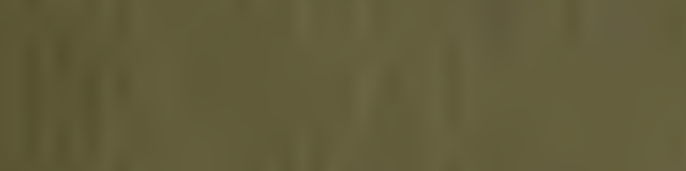

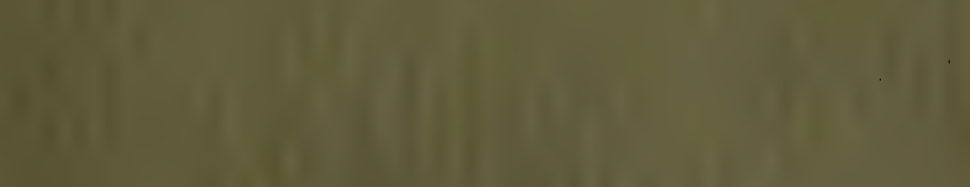

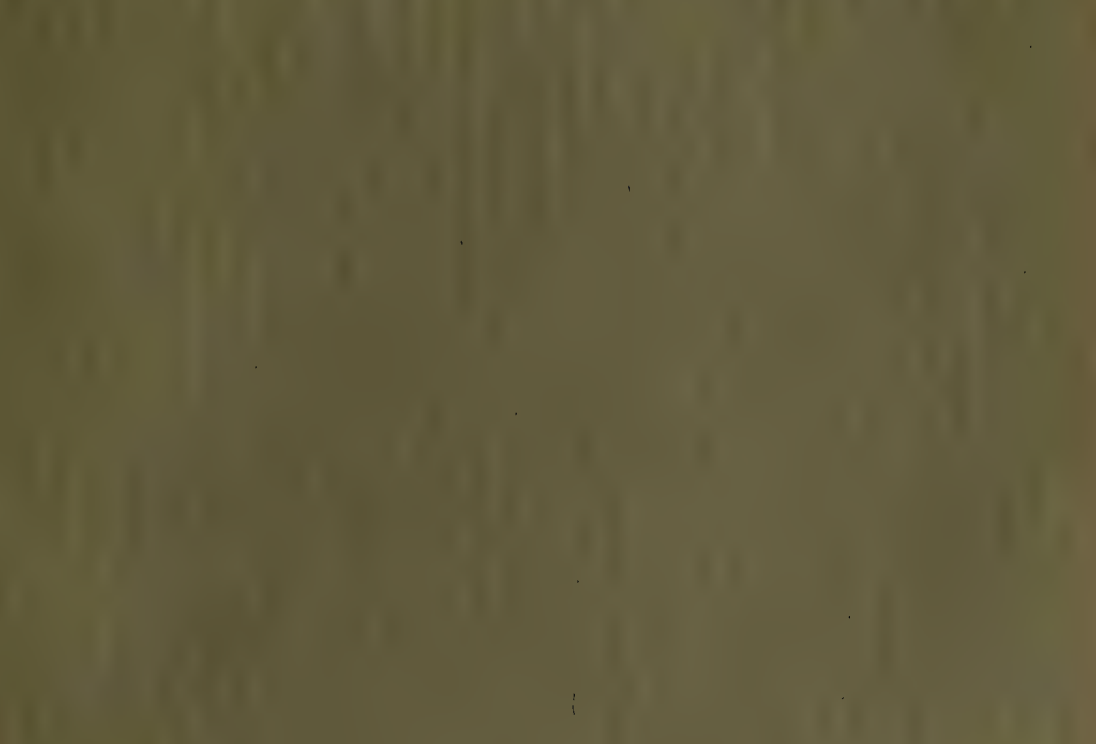




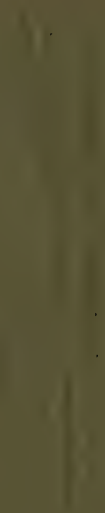

r

$$
\begin{aligned}
& \quad \\
& =\quad
\end{aligned}
$$






Fekete, Lajos/Die Verbreitung der forstl

Fekete, Lajos/Die Verbreitung der forst

35185001141975 
